\title{
Two-dimensional Polymer and Thin-Film Semiconductor-based Photonic Crystals for Biosensing Applications
}

\author{
Bashar M. Hamza \\ West Virginia University
}

Follow this and additional works at: https://researchrepository.wvu.edu/etd

\section{Recommended Citation}

Hamza, Bashar M., "Two-dimensional Polymer and Thin-Film Semiconductor-based Photonic Crystals for Biosensing Applications" (2011). Graduate Theses, Dissertations, and Problem Reports. 4729.

https://researchrepository.wvu.edu/etd/4729

This Thesis is protected by copyright and/or related rights. It has been brought to you by the The Research Repository @ WVU with permission from the rights-holder(s). You are free to use this Thesis in any way that is permitted by the copyright and related rights legislation that applies to your use. For other uses you must obtain permission from the rights-holder(s) directly, unless additional rights are indicated by a Creative Commons license in the record and/ or on the work itself. This Thesis has been accepted for inclusion in WVU Graduate Theses, Dissertations, and Problem Reports collection by an authorized administrator of The Research Repository @ WVU. For more information, please contact researchrepository@mail.wvu.edu. 


\title{
Two-dimensional Polymer and Thin-Film Semiconductor-based Photonic Crystals for Biosensing Applications
}

\author{
Bashar M. R. Hamza
}

\author{
Thesis submitted to the \\ College of Engineering and Mineral Resources \\ at West Virginia University \\ in partial fulfillment of the requirements \\ for the degree of \\ Master of Science \\ in \\ Electrical Engineering
}
Jeremy M. Dawson, Ph.D., Chair
Yuxin Liu, Ph.D
Lawrence A. Hornak, Ph.D

Lane Department of Computer Science and Electrical Engineering

Morgantown, West Virginia

2011

Keywords: photonic crystals, biosensor, fluorescence enhancement, polymer 


\begin{abstract}
Two-dimensional Polymer and Thin-Film Semiconductor-based Photonic Crystals for Biosensing Applications
\end{abstract}

Bashar M. R. Hamza

Detecting biomolecules at very low concentrations is of a significant importance for a wide variety of applications ranging from human health to national security. A diverse class of sensing platforms utilizing the specificity of physical properties of materials and their change in the presence of target analytes has been developed. The main objective of such systems is to deliver cost-effective, ultrasensitive, and reliable sensors that can withstand noisy environments (i.e. dirty samples) with efficient operational characteristics (low power, high throughput, etc.). Optical, electrochemical, and mechanical sensors have demonstrated promising detection capabilities, which further encouraged research aimed at producing even much more sensitive systems that are capable of extending detection limits to single molecules.

The unique optical properties of photonic crystals ( $\mathrm{PhCs}$ ) as well as their nano-meter scale features, which can be comparable to that of single molecules, make them well suited as a basis for sensors capable of fulfilling the ultra-sensitive detection requirements. Semiconductor materials are commonly used to engineer PhCs that can either trap light at high efficiency in high-quality factor resonant cavities to enhance fluorescence emission from labeled molecules, or cause a very precise attenuation of the transmitted or reflected light after the adsorption of unlabeled molecules to the surface of these $\mathrm{PhC}$ structures. However, the high cost of sensing platforms utilizing semiconductor materials motivates the development of soft lithographic techniques to fabricate photonic crystals in biocompatible polymer materials and simplify their integration with microfluidic channels and optical waveguides.

The theory, design, fabrication, and optical characterization of $\mathrm{PhC}$ lattice structures as biosensing platforms in both semiconductor and polymer materials will be demonstrated throughout this thesis. Electron Beam Lithography as well as soft lithographic techniques are presented to achieve submicrometer scale PhC lattices in silicon, Polydimethylsiloxane (PDMS), and epoxy. The main focus will be on a passive detection modality in which the $\mathrm{PhC}$ structures are used to manipulate light emitted from fluorescing molecules to achieve an enhancement of this emission. A 27-fold enhancement factor has been recorded when IR-emitting quantum dots were utilized as the emitting molecules within the PhCs. 
To my family . . . 


\section{ACKNOWLEDGEMENTS}

It is difficult to overstate my gratitude to my advisor, Dr. Jeremy M. Dawson, whose enthusiasm, inspiration, and continuous guidance have been invaluable for my Master's thesis work. I will never forget his selfless support that has always made me appreciate having him as my mentor. I also wish to thank my committee members, Dr. Yuxin Liu and Dr. Lawrence Hornak, whose support, helpful advice, excellent teaching, and lots of amazing new ideas have been very critical to my work.

Dr. Lloyd Carroll's advice and help with the polymer nano-molding along with Dr. Yuxin Liu's extensive experience in microfluidics deserve my special appreciation as well. They both dedicated many hours to teach me and opened their labs for my experiments and for that I am very thankful. I am also thankful to Dr. Cerasela-Zoica Dinu and Dr. Letha Sooter for their help to explain the Biologybackground that I needed for this work.

I would also like to thank Dr. Dimitris Korakakis for his support and guidance during my undergraduate research experience. Moreover, I would like to thank Dr. Kolin Brown, Dr. Weiqiang Ding, Mr. Harley Hart, the SRF staff, and Dr. Xian Cao for their great help with cleanroom-based microand nano-scale fabrication both as an undergraduate and a graduate student.

My gratitude extends to the current graduate students. In particular, I would like to thank Anand Kadiyala, Lee Rodak, Rohit Guswami, Vamsi Kumbham, Ronak Rahimi, Joshua Justice, Vishal Narrang, Sriniya Musonuro, and Dr. Kyoungnae Lee. They have been an amazing group of researchers that I enjoyed working with and learning from. I would also like to thank the current and previous undergraduate students for their contributions to the project. Those are: Jacob Kinard, Terrance Yarber, Jason Okerman, Ayesha Mazumdar, Matthew Simmons, Jason Jaxon, Jared Crowford, Caroline Kilemi, Brad Potteiger, Kathryn Smith, Alicia Harmon, and Chloe Snyder. I have also had the opportunity to meet and be advised by past graduate students throughout my undergraduate and graduate level studies. Of those, I would like to thank: Joshua Nightingale and Dr. Randy Tomkins for their help with the initial photonic crystals' work, Vincent Pagan, Rich Farrel, Adam Kabulski, Kalyan Reddy, Dr. Yi Yang, and Satish Yeldandi for their help and advice with research and fabrication, Hyma Yalamanchili and Henry Andagana for their significant help with the modeling and fabrication work of photonic crystals and to Dr. Sridhar Kuchibhatla for his knowledge in all aspects of research.

Lastly, and most importantly, I would like to thank my parents and my sisters. They have always supported me, taught me, and loved me like no other son or brother. To them I dedicate this thesis.

Bashar M. R. Hamza

July $3^{\text {rd }}, 2011$

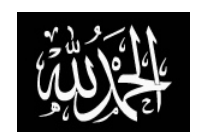




\section{TABLE OF CONTENTS}

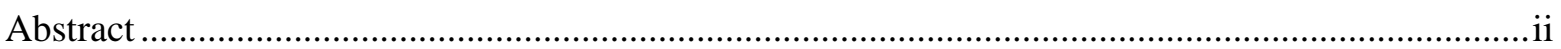

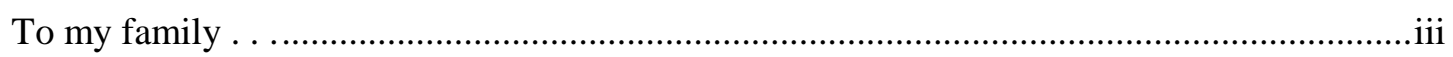

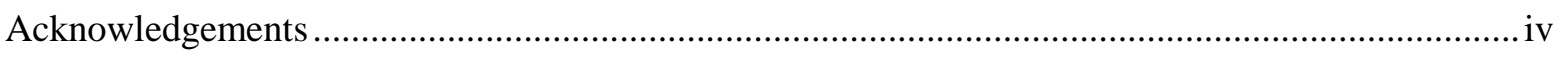

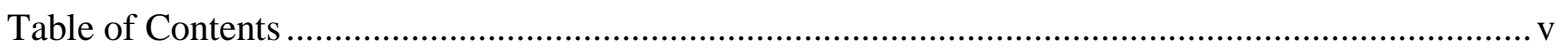

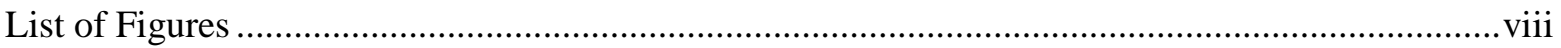

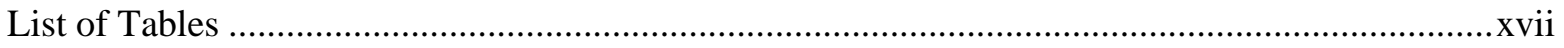

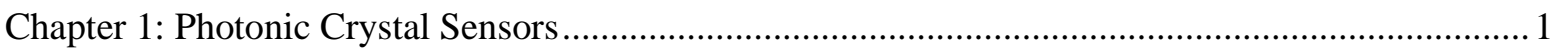

1.1 Photonic Crystals and Their Applications.................................................................. 1

1.2 The active Detection Modality utilizing Photonic Crystals ............................................. 3

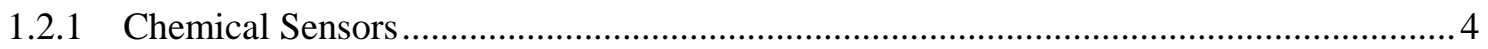

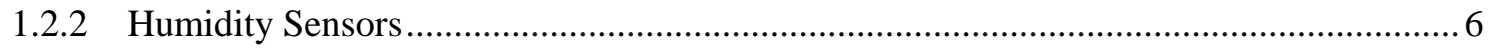

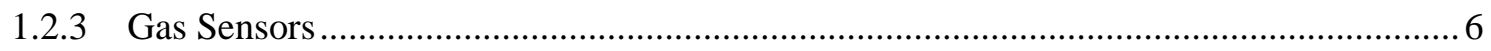

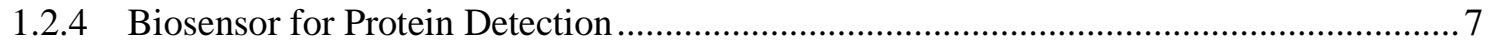

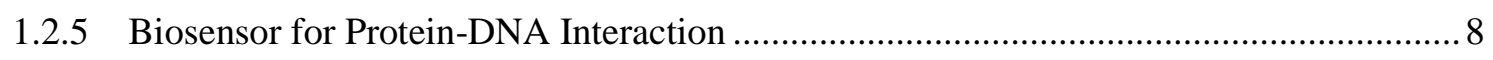

1.3 The Passive Detection Modality utilizing Photonic Crystals .......................................... 10

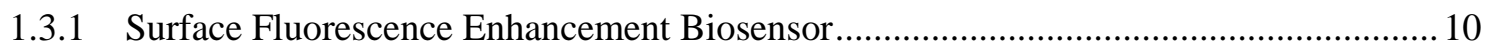

1.3.2 Resonant-Cavity-Based Fluorescence Enhancement Biosensor ................................... 13

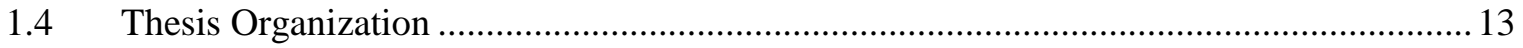

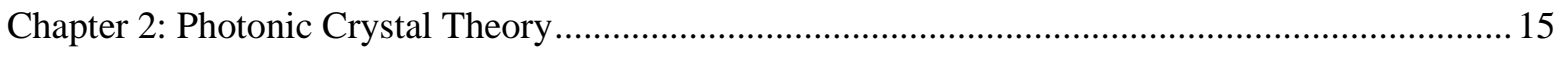

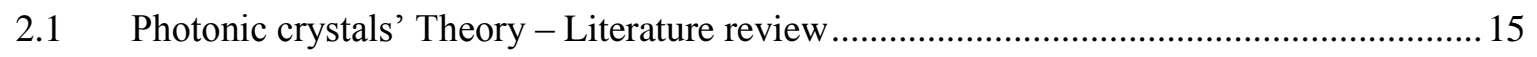

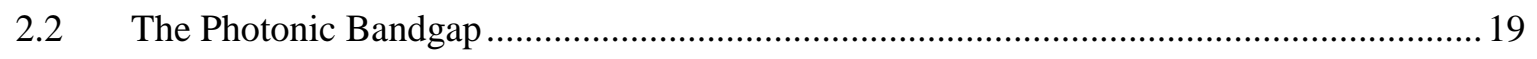

2.2.1 Two-dimensional Photonic Crystals (Infinitely Long) .............................................. 24

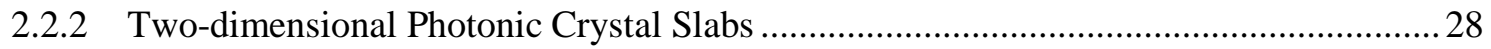

2.2.2.1 Defects in Two-dimensional Photonic Crystals ........................................................ 31

2.3 Photonic Crystal as Core Structures of a Biosensor..................................................... 33 
Chapter 3: The Modeling of Photonic Crystal LAttices

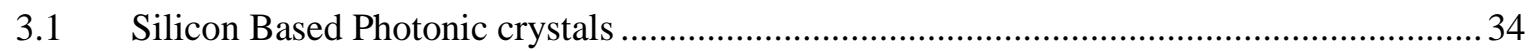

3.1.1 Infinitely-Thick Photonic Crystal Lattices of Silicon and Toluene ............................... 35

3.1.2 Finite-Thickness Slab Photonic Crystals of Silicon, Toluene, and Air..........................50

3.1.3 Time Domain Modeling of a Slab Photonic Crystal Lattice .........................................61

3.2 poly(dimethylsiloxane) (PDMS)-based Photonic Crystals .............................................65

3.2.1 Analyzing Infinitely-Thick Photonic Crystal Lattices of PDMS and Air ...................... 70

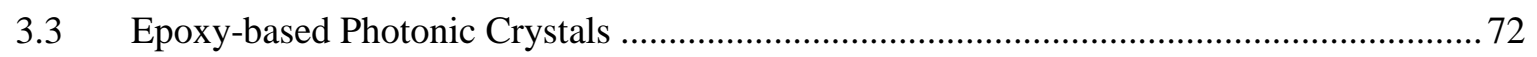

3.3.1 Analyzing Infinitely-Thick Photonic Crystal Lattices of Epoxy and Air ...................... 77

Chapter 4: Fabrication of Semiconductor- and Polymer-based Photonic Crystal Lattices .................79

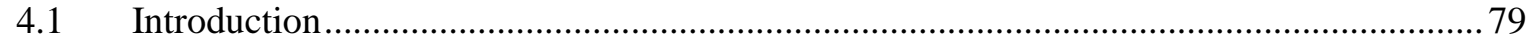

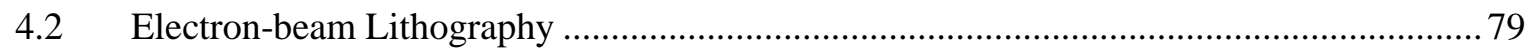

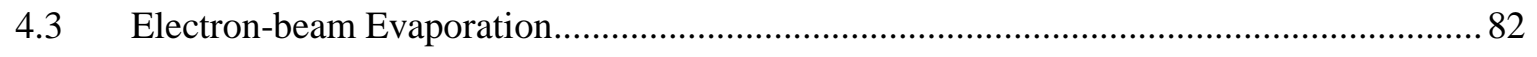

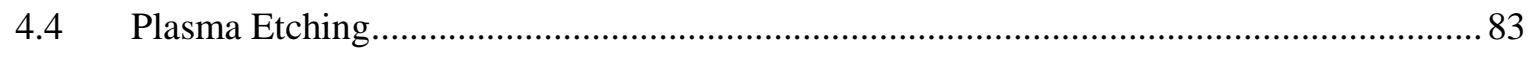

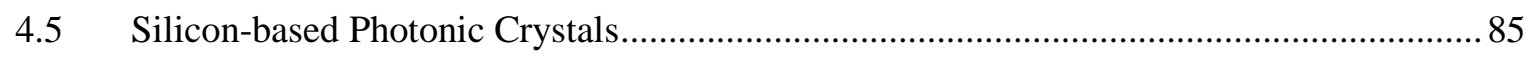

4.5.1 Fabrication of Symmetric Photonic Crystal Lattices of Air Holes in Silicon ................. 86

4.5.2 Fabrication of Symmetric Photonic Crystal Lattices of Silicon Pillars and Air .............92

4.5.3 Hydrogen-Silsesquioxane (HSQ) Fabrication Recipe ................................................97

4.6 Polymer-Based Photonic Crystals .......................................................................... 107

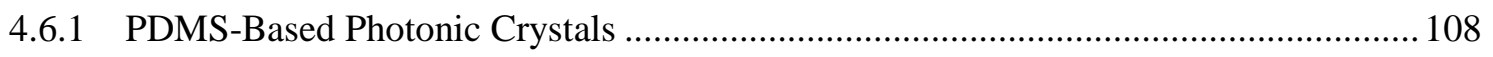

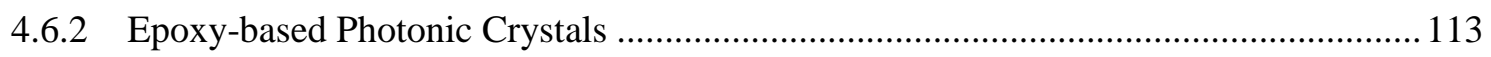

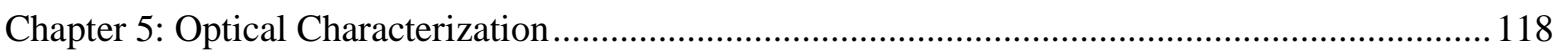

5.1 Characterization of a Finite-thickness slab of a triangular lattice of toluene-filled holes in Silicon $\quad 121$

5.2 Characterization Finite-thickness Photonic crystal slabs of a triangular lattice of Si Pillars

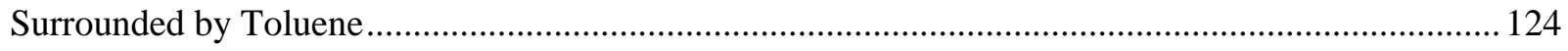

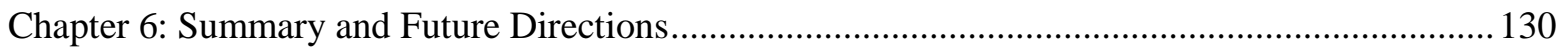


4.1. Future Directions for Finite-thickness Semiconductor-based Photonic crystals.............. 130

4.2. Future Directions for the polymer-based photonic crystals ........................................ 131

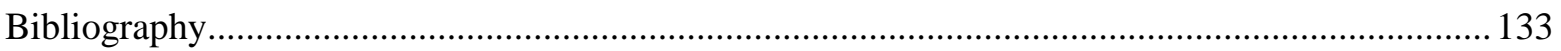

Appendix A: E-beam Lithography Cad File Process .................................................................... 139

Appendix B: E-beam Lithography Process …....................................................................... 148

Appendix C: Reducing Charging Effects when imaging polymer-based Photonic Crystals ............ 155

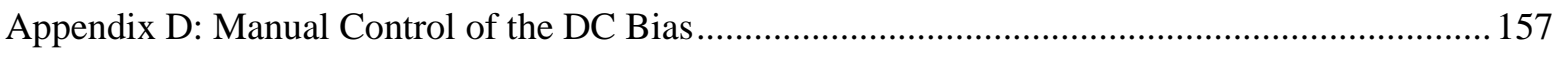




\section{LIST OF FIGURES}

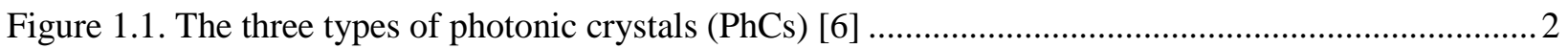

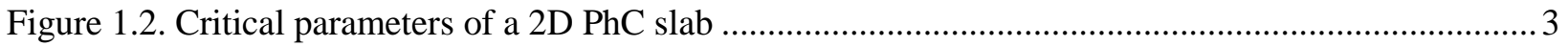

Figure 1.3. An example of a chemical sensor for $\mathrm{pH}$ changes in a solution. As the volume of the 3D polymer-based $\mathrm{PhC}$ changes, red or blue shifts can be observed accordingly [13] ..................................5

Figure 1.4. Examples of two 3D PhC based glucose sensing mechanisms. (Top) Changing lattice constant due to volume-induced changes. (Bottom) changing the radius due to swollen microgel particles chemical sensor for glucose using the three-dimensional PCCAs [17] .................................................................

Figure 1.5. (Left) SEM image of 3D PhC-based hydrogel humidity sensor. (Right) Spectra shift with time from ambient conditions $50 \%$ to $80 \% \mathrm{RH}[18]$. 6

Figure 1.6. (a) Scanning electron microscopy image of the cavity (b) Transmission of a PhC resonator in vacuum (solid black line), nitrogen (dashed red line), and SF6 (blue dotted line) [21] 7

Figure 1.7. Schematic of bio-molecule recognition: (a) the target molecules are captured by the probe molecules. (b) The bio-molecules form a uniform layer on the internal surface of the sensor. In reality the layer thickness is very small compared with the pore size. [23]

Figure 1.8. Normalized transmission spectra of the PC microcavity. Curve (a) indicates the initial spectrum resonance after oxidation and silanization, curve (b) is measured after glutaraldehyde attaches to the pore walls, and curve (c) is obtained after infiltration of BSA molecules. [23]

Figure 1.9. (a) Schematic of the PC biosensor. A broadband LED illuminates the biosensor from the bottom, and reflected light is collected and transferred to a spectrometer where the PWV is measured. (b) Image of PC biosensor films adhered to the bottom of black 384-well plates. (c) Diagram of protein-DNA binding experiments performed with PC biosensors. Streptavidin-coated biosensors are used to bind biotinylated DNA oligomers, and a distinct peak wavelength of the reflected light is observed. After the addition of Starting Block (Pierce Biotechnologies), a DNA-binding protein is added, and a shift in the wavelength of reflected light is observed [26].....

Figure 1.10. (a) Atomic force microscopy image of a portion of the photonic crystal surface. (b) Side view of the model used in Rigorous Coupled-Wave Analysis computations of the structure response. (c) Simulated transmission response as a function of wavelength under TE illumination for a region of the structure resonant at $633 \mathrm{~nm}$. (d) Simulated electric field intensity for the structure in (b) when illuminated with $633 \mathrm{~nm}$ TE-polarized light at normal incidence. [37]. 12 Figure 1.11. Fluorescence enhancement on the surface of a 1D PhC with nano-rods as shown in image. inset [38] 
Figure 1.12.( Top) two and three dimensional representation of the fluorescence enhancement caused by the spatial confinement of the emitted light within the PhC. The resonant cavity is carefully designed to cause the emitted light from the labeled molecule to be trapped and leaked toward a detector above the PhC slab. (Bottom) Finite Difference Time Domain (FDTD) [46] ......................................................... 14 Figure 2.1. A $1 \mathrm{D}$ photonic crystal with varying dielectric constant in the $y$ direction. Red layers can represent high refractive index regions while blue layers can represent low refractive index regions 19 Figure 2.2. TE band diagram of a two-dimensional infinitely-thick photonic crystal of triangular lattice of air holes in silicon. Green band indicate the region of no solutions (photonic bandgap)..... 22 Figure 2.3. The primitive lattice vectors, the reciprocal lattice vectors, and the Brillouin zones for the

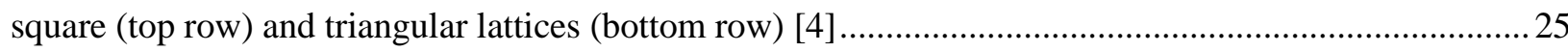

Figure 2.4. Infinitely-long $2 \mathrm{D} \mathrm{PhC}$ with a triangular lattice symmetry of dielectric rods .......................26 Figure 2.5. PWEM solved using MIT Photonic Bands (MPB) to obtain the dispersion (band) diagram for two different lattices. (top) Square lattice of silicon rods surrounded by toluene and (bottom) triangular lattice of silicon rods surrounded by toluene. Bandgap can...............................................................2 27

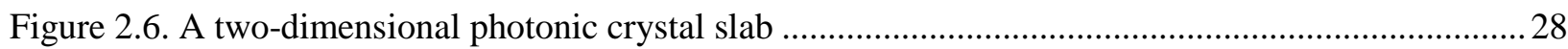

Figure 2.7. TE band diagram of a triangular lattice of an infinitely-long photonic crystal of toluene holes in $\mathrm{Si}$ (top)and a triangular lattice of a slab photonic crystal of toluene holes in Si (bottom). Photonic bandgaps are shaded in green..... 30

Figure 2.8. (a) a resonant cavity in which the defect is generated by filling in the holes with high dielectric material surrounding a central lower dielectric circular opening. (b) a resonant cavity in which 6 holes on top and bottom of a central hole are filled with high dielectric region. Extending this filling technique to the edge of the photonic crystal can generate a W1 waveguide. .33 Figure 3.1. (Left) A representation of an infinitely-thick photonic crystal lattice of air holes in silicon. (Right) TE band diagram of an infinitely-thick photonic crystal of toluene holes in Silicon. Point $\mathrm{x}$ is the top of the lowest band while point y represents the bottom of the second band. The difference is the

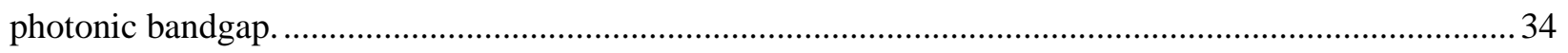

Figure 3.2. TM band diagram of a square lattice of toluene holes in Si. A very narrow bandgap is indicated with the red arrow between the $8^{\text {th }}$ and $9^{\text {th }}$ bands .

Figure 3.3. TM bandgap map for a square lattice of Si pillars in toluene. The widest observed bandgap was at $\mathrm{r} / \mathrm{a}=0.24$. .38

Figure 3.4. TE bandgap map for a square lattice of Si pillars in toluene. The two bandgaps at $\mathrm{r} / \mathrm{a}=0.24$ are indicated by a vertical line. .38

Figure 3.5. TM band diagram for $\mathrm{r} / \mathrm{a}=0.24$ with an $18.57 \%$ bandgap width between the first two bands 
Figure 3.6. TE band diagram for $\mathrm{r} / \mathrm{a}=0.24$ with an $5.076 \%$ bandgap width between the $9^{\text {th }}$ and $10^{\text {th }}$ bands .39

Figure 3.7. TM bandgap map of a triangular lattice of toluene holes in silicon. The widest observed bandgap was at $\mathrm{r} / \mathrm{a}=0.39$ 40

Figure 3.8. TM bandgap map of a triangular lattice of toluene holes in silicon. The widest observed bandgap was at $\mathrm{r} / \mathrm{a}=0.4$ 40

Figure 3.9. TM band diagram demonstrating two bandgaps between the higher-order bands (6 and 7, and 8 and 9) for a triangular photonic crystal lattice of toluene holes in silicon with $\mathrm{r} / \mathrm{a}=0.39$

Figure 3.10. TE band diagram demonstrating a bandgap of size $29.59 \%$ between the first two bands for a triangular lattice of toluene holes in silicon with $\mathrm{r} / \mathrm{a}=0.4$

Figure 3.11. TM bandgap map for a triangular lattice of silicon pillars surrounded by toluene. The widest observed bandgap for this lattice was found at $\mathrm{r} / \mathrm{a}=0.22$. .42

Figure 3.12. TE bandgap map for a triangular lattice of silicon pillars surrounded by toluene. The widest observed bandgap for this lattice was found at $\mathrm{r} / \mathrm{a}=0.2$. .42

Figure 3.13. TM Band diagram of a triangular lattice of silicon pillars in toluene with $\mathrm{r} / \mathrm{a}=0.2$....... 43

Figure 3.14. TE band diagram of the largest TE bandgap between the $4^{\text {th }}$ and $5^{\text {th }}$ bands for a triangular lattice of silicon pillars surrounded by toluene with $\mathrm{r} / \mathrm{a}=0.2$.

Figure 3.15. TE-like band diagram for a square lattice of Si pillars in Toluene demonstrating no bandgap within the guided modes below the light cone can be observed.

Figure 3.16. Zodd (TM-like) band diagram of a slab photonic crystal $(\mathrm{h}=1.2 a)$ square lattice of silicon pillars immersed in toluene. Only a directional bandgap can be observed between the X and M points...52 Figure 3.17. Zeven (TE-like) band diagram of a slab photonic crystal square lattice $(\mathrm{h}=0.5 a)$ of silicon pillars in toluene. No bandgap can be resolved under the light cone for the guided modes..... .52 Figure 3.18. Zodd (TM-like) band diagram of a slab photonic crystal $(\mathrm{h}=0.5 a)$ square lattice of silicon pillars in toluene. Neither direction nor a complete bandgap can be observed for the guided modes........53 Figure 3.19. Zeven (TE-like) band diagram of a slab photonic crystal triangular lattice $(\mathrm{h}=1.2 a)$ of silicon pillars in toluene. No bandgap can be resolved under the light cone for the guided modes.

Figure 3.20. Zodd (TM-like) band diagram of a slab photonic crystal triangular lattice $(\mathrm{h}=1.2 a)$ of silicon pillars in toluene. Only a directional bandgap can be resolved in the $\mathrm{M}$ to $\mathrm{K}$ direction. .54 Figure 3.21. Zeven (TE-like) band diagram of a slab photonic crystal triangular lattice $(\mathrm{h}=0.5 a)$ of silicon pillars in toluene. No bandgap can be resolved under the light cone for the guided modes .54 Figure 3.22. Zodd (TM-like) band diagram of a slab photonic crystal triangular lattice $(\mathrm{h}=0.5 a)$ of silicon pillars in toluene. No bandgap can be resolved under the light cone for the guided modes. .55 
Figure 3.23. Zeven (TE-like) band diagram of a slab photonic crystal triangular lattice $(\mathrm{h}=1.2 a)$ of toluene holes in silicon. Only a directional bandgap between $\mathrm{M}$ and $\mathrm{K}$ can be resolved for the guided modes.....56 Figure 3.24. Zodd (TM-like) band diagram of a slab photonic crystal triangular lattice $(\mathrm{h}=1.2 a)$ of toluene holes in silicon. Only a very narrow directional bandgap between $\mathrm{M}$ and $\mathrm{K}$ can be resolved for the guided modes. .56

Figure 3.25. Zeven (TE-like) band diagram of a slab photonic crystal triangular lattice $(\mathrm{h}=0.5 a)$ of toluene holes in silicon. Only a directional bandgap between $\mathrm{M}$ and $\mathrm{K}$ can be resolved for the guided modes.....57 Figure 3.26. Zodd (TM-like) band diagram of a slab photonic crystal triangular lattice ( $\mathrm{h}=0.5 \mathrm{a}$ ) of toluene holes in silicon. Only a directional bandgap between $\mathrm{M}$ and $\mathrm{K}$ can be resolved for the guided modes.....57 Figure 3.27. TE bandgap for a triangular lattice of slab photonic crystal of toluene holes in silicon sandwiched by air background.. .58

Figure 3.28. TE band diagram for a triangular slab photonic crystal lattice of toluene holes in silicon sandwiched by air. A bandgap of size $13.4 \%$ can be observed.

Figure 3.29. TM band diagram for a triangular slab photonic crystal lattice of toluene holes in silicon sandwiched by air. A bandgap of size $4.8 \%$ can be observed between the $4^{\text {th }}$ and $5^{\text {th }}$ bands .59 Figure 3.30. TE band diagram of a finite thickness slab of a triangular lattice of toluene holes in Silicon sandwiched by air $(\mathrm{h} / \mathrm{a}=0.7 \mathrm{r} / \mathrm{a}=0.35)$.

Figure 3.31. Electric field concentration in the plane of the photonic crystal for three frequencies: 0.278, .015 , and 0.47 .

Figure 3.32. Light confinement within a L7 defect for frequencies that fall inside the bandgap only ....... 64

Figure 3.33. Total flux as a function of normalized frequency.....

Figure 3.34. TE and TM bandgap map for a square lattice of air holes in PDMS demonstrating no bandgap for radii between $0.2 a$ and $0.4 a$. .66

Figure 3.35. TE and TM bandgap map for a square lattice of PDMS pillars in air demonstrating no bandgaps for radii between $0.2 a$ and $0.4 a$

Figure 3.36. TM bandgap map for a triangular lattice of air holes in PDMS demonstrating no bandgaps for radii range between $0.2 a$ and $0.4 a$......

Figure 3.37. TE bandgap map for a triangular lattice of air holes in PDMS .68

Figure 3.38. TE band diagram of a triangular lattice of air holes in PDMS demonstrating a narrow bandgap $(1.304 \%)$ between the first and second bands for a lattice with $\mathrm{r} / \mathrm{a}=0.36$. .68

Figure 3.39. Bandgap map of a triangular lattice of PDMS pillars in air. A $4.461 \%$ bandgap at $\mathrm{r} / \mathrm{a}=0.28$ can be observed. .69

Figure 3.40. TE bandgap map for a triangular lattice PDMS pillars in air. No bandgap is observed. .69 
Figure 3.41. TM band diagram of a triangular lattice of PDMS pillars in air demonstrating a bandgap of $4.461 \%$ at $\mathrm{r} / \mathrm{a}=0.28$

Figure 3.42. TM and TE bandgap map for a square lattice of Air Holes in Epoxy demonstrating no bandgaps of any size for the radii range between $0.2 a$ and $0.4 a$ .73

Figure 3.43. TM and TE bandgap map for a square lattice of Epoxy pillars in air demonstrating no bandgaps of any size for the radii range between $0.2 a$ and $0.4 a$ .73

Figure 3.44. TM band diagram of a triangular lattice of air holes in Epoxy. No bandgap was observed for the radii range between $0.2 a$ and $0.4 a$ .74

Figure 3.45. TE band diagram of a triangular lattice of air holes in Epoxy. A bandgap of size $4.41 \%$ was observed for a lattice with $\mathrm{r} / \mathrm{a}=0.37$ .74

Figure 4.1. The electron optical components inside the column of an EBL system [57] ..... .81

Figure 4.2. E-beam Evaporation system components [59] .83

Figure 4.3. Schematic of plasma etching [61] 84

Figure 4.4. A tilted SEM image demonstrating the different layers of an SOI wafer. Top right corner inset demonstrates a description of the thickness of each layer . 86

Figure 4.5. The negative-tone e-beam resist pattern development mechanism [64]..... 87

Figure 4.6. Process flow of a suspended $\mathrm{PhC}$ in silicon as adapted from [43]. (1) ma-N 2403 was first spun coated on the SOI sample and an EBL procedure was carried out to define a pattern in the resist. (2) After developmen in ma-D 525 for 65 seconds, pillars of ma-N were resolved. (3) A thin layer of Ni was then evaporated on the surface. (4) A lift-off process was then done to obtain the desired pattern in the Ni hard mask. (5) $\mathrm{A} \mathrm{CF}_{4} / \mathrm{O}_{2}$ plasma etch was then carried out to transfer the pattern to the underlying $\mathrm{Si}$ layer. (6) A 15-second dip in HF solution was then done to open the underlying channel and achieve the desired symmetry above and below the slab.

Figure 4.7. SEM image of the suspended $\mathrm{PhC}$ structures in silicon with underlying air channels. Image was captured at $85^{\circ}$ glancing angle

Figure 4.8. A high magnification image of the $\mathrm{PhC}$ structure in silicon and the underlying channel. Image was captured at $80^{\circ}$ glancing angle

Figure 4.9. Thick Ni layers can cause serious problems when trying to do the lift off process. In this image, the Ni remained on top of the ma-N pillars even after a sonication step. .91

Figure 4.10. SEM image demonstrating an over sonication result. This will cause the thin Ni layers to break and not remain attached to the Si to act as a hard etching mask. .91

Figure 4.11. Collapsed PhC structures due to a drying step with a high Nitrogen flow .92

Figure 4.12. Positive-tone e-beam resist exposure and development mechanism [64] .93 
Figure 4.13. . Process flow of a symmetric PhC of pillars in air. (1) electron beam lithography in the PMMA resist. (2) development of the resist in 1:3 (MIBK:IPA) for 65 seconds. (3) E-beam Evaporation of a thin Ni layer $(20 \mathrm{~nm})$. (4) Metal lift off in Remover PG for 15 minutes followed by a two second sonication (5) Dry etch of the silicon using a $\mathrm{CF}_{4} / \mathrm{O}_{2} 27 / 3$ gas mixture at $-85 \mathrm{~V}$ DC bias followed by a $\mathrm{Ni}$

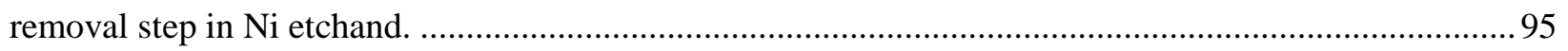

Figure 4.14. A tilted SEM image of the final structure of Silicon Pillars surrounded by air.....................96

Figure 4.15. SEM of an over-etched Si sample in a $\mathrm{CF}_{4} / \mathrm{O}_{2}(27 / 3)$ gas mixture for 4 minutes ................96

Figure 4.16. The cage-like structure of an HSQ molecule [65] ..........................................................97

Figure 4.17. Spin speed vs. thickness in nanometer for HSQ (FOx-14) ...............................................99

Figure 4.18. SEM images demonstrating the exposure-recipe optimization patterns. Dose range is from $100-600 \mu \mathrm{C} / \mathrm{cm}^{2}$ and development time is 11 minutes.

Figure 4.19. SEM images of the desired range of doses over different development times; 5, 8, 12, and 18

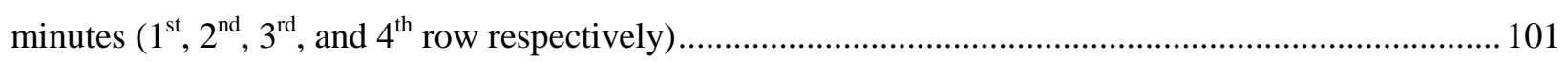

Figure 4.20. SEM images showing (left) a $90^{\circ}$ cross-section image of a $315 \mu \mathrm{C} / \mathrm{cm}^{2}$ dose of HSQ on silicon and 12-minute development. (Right) A top view of the pattern................................................ 102 Figure 4.21. SEM images showing the final structure Si pillar etched using HSQ as a hard mask. Left column demonstrates images of etched silicon using a $27 / 3\left(\mathrm{CF}_{4} / \mathrm{O}_{2}\right)$ etch recipe while the right column shows SEM images of etched Si using a 18/2 $\left(\mathrm{CF}_{4} / \mathrm{O}_{2}\right)$ etch recipe ................................................... 103 Figure 4.22.Inverse pattern writing technique using single unit stitching method when creating the

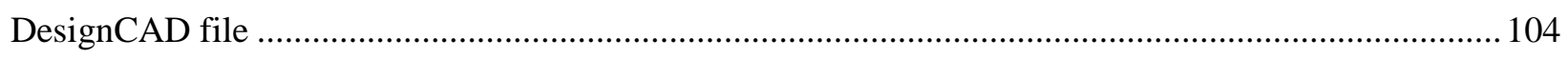

Figure 4.23. SEM images of the inverse HSQ patterns demonstrating the fully and underdeveloped structures 105

Figure 4.24. Tilted SEM images of the final fabricated PhC structures in silicon using HSQ as a mas. (1) $70^{\circ}$ angle (2) $80^{\circ}$ angle 105

Figure 4.25. (1) Tilted SEM image of the Si pillars that were achieved when using maN as a hard etching mask. (2) The PhC structure in silicon using PMMA as a hard etching mask....................................... 106 Figure 4.26. Fabrication procedure to achieve a final photonic crystal lattice of air holes in PDMS. (1) PMMA spin coating, (2) e-beam lithography, (3) PMMA development, (4) Ni thin mask deposition, (5) $\mathrm{Ni}$ lift off and dry etch of $\mathrm{Si}$, (5) removal of Ni mask. (6) PDMS molding, (7) final PhC structure in PDMS. 109

Figure 4.27. Optical and SEM images of the Si master (left column images) and the molded PDMS sample (right column images) containing the sub-micron PhC lattice. 110

Figure 4.28. Un-resolved $\mathrm{PhC}$ lattice of air holes due to the use of low vacuum during the degassing step 
Figure 4.29. SEM images of the Si master mold containing pillars with $\mathrm{r}=150 \mathrm{~nm}, \mathrm{a}=450 \mathrm{~nm}$ (spacing of $150 \mathrm{~nm}$ ), and $\mathrm{h}=460 \mathrm{~nm}$. (1) top view. (2) tilted image of the full block, (3) the tips of the Si pillars, and (4) the full Si pillars

Figure 4.30. SEM images of the 5 molded PDMS samples using a single Si master. Observed distortions are due to charging effects and cracking of PDMS during the sputtering step. For $5^{\text {th }}$ mold, a top view of the full pattern area is shown 112 Figure 4.31 (1) PMMA resist spin coating on the first Si mold, (2) E-beam Lithography, (3) Development of the PMMA resist, (4) Ni mask deposition using E-beam Evaporation, (5) Ni mask lift off and dry etch of the Si substrate, (6) Removal of the Ni mask layer, (7)-(8) molding of the PDMS to create the inverse pattern, (9) Pouring Epoxy on the PDMS master and curing it in an oven set to 60C for 5 hours (10) Final PhC structure in Epoxy. .115

Figure 4.32 SEM images of the Si master mold. (1) The full PhC block (2) Top image with lattice parameters. (3) A $70^{\circ}$ angle image of the full pillars .116

Figure 4.33 (1)-(2) SEM images of the final sub-micron PhC lattice of epoxy pillars surrounded by air, (3)-(4) SEM images of the final sub-micron PhC lattice of air holes in epoxy when a Si master with holes is used instead of pillars. 116

Figure 4.34 (1) Un-resolved epoxy pillars due to the use of low vacuum during the degassing step (2) resolved Epoxy pillars due to the use of a XD-5 Edwards scroll pump during the degassing step. .117 Figure 4.35 SEM images of the 5 molded epoxy samples using a single Si and PDMS master molds. Observed distortions are mainly due to charging effects. For the $5^{\text {th }}$ mold, a top view of the final molded $\mathrm{PhC}$ area is shown. .117

Figure 5.1. The absorption (1) and emission spectra (2) of the PbS QDs............................................. 120

Figure 5.2. Optical setup to observe fluorescence enhancement

Figure 5.3. Band diagrams of (1) an infinitely-thick $\mathrm{PhC}$ and (2) finite-thickness slab with $\mathrm{r} / \mathrm{a}=0.35$ and $\mathrm{h} / \mathrm{a}=0.9$

Figure 5.4. SEM image of the suspended PhC structures. Top rows are identical $\mathrm{PhC}$ lattices designed for $1100 \mathrm{~nm}$ to be within their bandgap. Middle row are identical $\mathrm{PhC}$ lattices in which the $1100 \mathrm{~nm}$ emission falls below their bandgap. Bottom row demonstrates a repeated, identical random lattice with no bandgap.

Figure 5.5. Optical characterization results of a (1) suspended and (2) an un-suspended PhC lattices of holes in Si fabricated in SOI and Si samples, respectively. (3) The background image of an area close to the PhC lattice. (4) SOI after background subtraction. (5) Si (unsuspended) after background substraction 
Figure 5.6. (1) The band diagrams of a finite-thickness slab of $\mathrm{Si}$ pillars surrounded by toluene background (2) A two dimensional cross-section representation of the PhC structure with the underlying $\mathrm{SiO}_{2}$-Toluene effective dielectric region.

Figure 5.7. SEM images of the fabricated PhC lattices fabricated in one field of view for characterization. (Top) SEM image showing the four different lattices with boxes numbered 1 through 4. (1) SEM image for the structure of box No. 1 with a photonic bandgap surrounding the $1100 \mathrm{~nm}$ emission. (2) Triangular lattice with $\mathrm{r} / \mathrm{a}=0.2$ and no bandgap surrounding the $1100 \mathrm{~nm}$. (3) A random square lattice with no bandgap. (4) A random triangular lattice with no bandgap. Insets are zoomed-in images of each lattice. 126

Figure 5.8. Cropped optical image of the (1) PhC lattices region as well as the (2) background and the (3) final image after background subtraction. Red boxes indicate regions where other $\mathrm{PhC}$ are fabricated with no bandgaps and hence no enhancement.

Figure 5.9. Optical images as captured by a Goodrich IR camera of the region containing the PhC lattices as toluene evaporated. (1) After 30 seconds of placing the sample on the toluene droplet. (2) After 1 minute. (3) After 5 minutes. (4) After 10 minutes 128

Figure 5.10. The different PhC lattice as they appear after the evaporation of the QD solution and after the removal of the background removal. All lattices appear to be brighter due to the aggregation of the QDs within the pillars 128

Figure 5.11. (Top) Optical image captured using the Goodrich IR camera with the MATLAB line profile in yellow. (3) Pixel intensity profile as plotted using MATLAB indicating the average peak height of 61

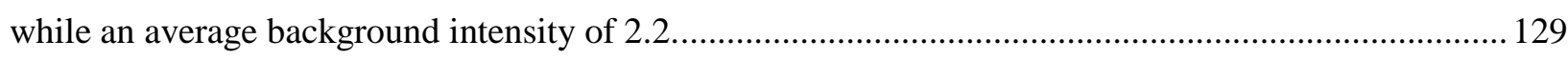

Figure A-1. Project selection on NPGS. Red arrow indicating the current project directory. .................. 139

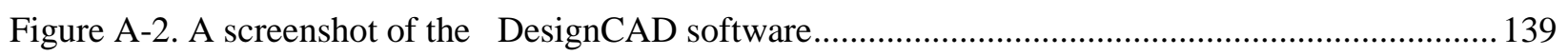

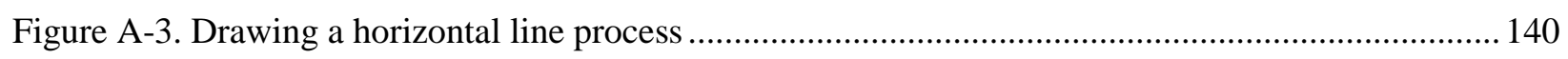

Figure A-4. Drawing a horizontal line and giving its length which is normally the lattice constant when

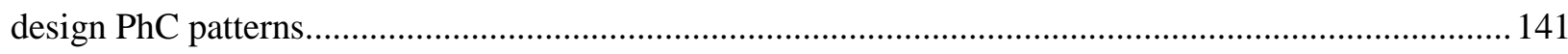

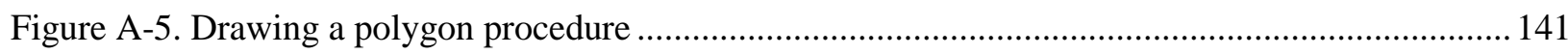

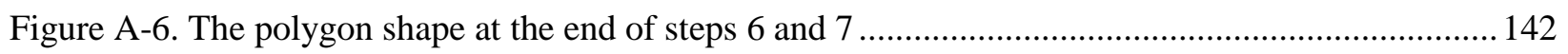

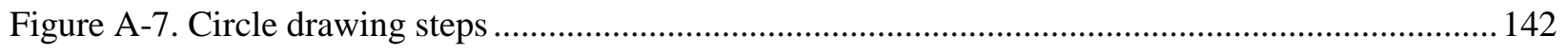

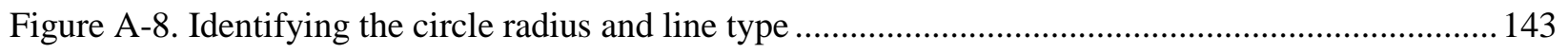

Figure A-9. 'Make Array' procedure. Red arrow indicates where the MA icon can be found. Left image indicates the first click below the bottom left corner of the feature and the right image indicates the

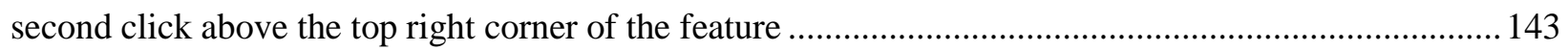

Figure A-10. Entering the number of columns of the desired array .................................................... 144 


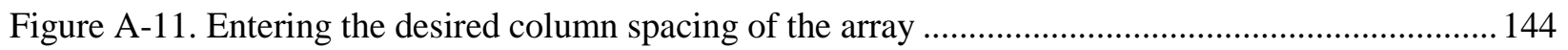

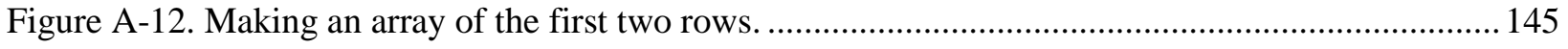

Figure A-13. One triangular lattice PhC block ................................................................................. 145

Figure A-14. Making an array of the PhC block for different doses. .................................................. 146

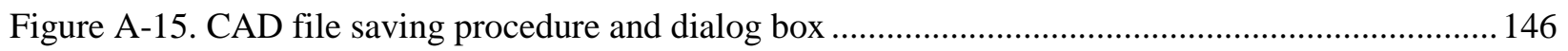

Figure A-16. MaxMag procedure of step 20. Left image indicates the location of the MaxMag command in the NPGS drop down menu. The right image indicates the dialog box that appears when the NPGS software finishes the MaxMag calculation of the designed pattern ...................................................... 147

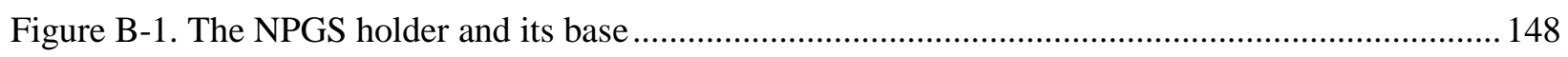

Figure B-2. Creating a small scratch in the edge of the sample for focusing purposes. ........................ 148

Figure B-3. Sample loading procedure. Red arrows indicate vent icon, the specimen chamber latch, and

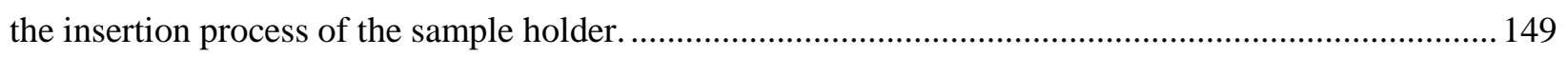

Figure B-4. Procedure to insert the sample holder into the main column............................................. 150

Figure B-5. Turning on the beam blanker by pushing the button indicated in the red arrow of the left image. Right image indicates the first image of the Faraday cup once the beam is turned ON in LM mode.

Figure B-6. Checking the probe current by collecting the beam inside the Faraday cup ....................... 151

Figure B-7. The scratch area and the particle that will be used for focusing ....................................... 152

Figure B-8. Image of the Specimen Stage Controller indicating the + and $-Z$ buttons ......................... 152

Figure B-9. The particle in SEM mode before and after the initial focusing step using the + or $-\mathrm{Z}$ buttons.

Figure B-10. Spot burning procedure to observe the beam shape.

Figure B-11. A critical step of the unloading procedure in which the rod has to remain in a horizontal state when being pulled out until it passes the white arrow marks .............................................................. 154

Figure C-1. Placing the SEM golden cylinder on a sputter station metal plate ..................................... 155

Figure C-2. Cutting the polymer sample to almost the size of the SEM golden cylinder........................ 155

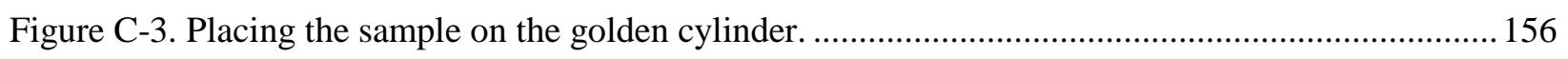

Figure C-4. The final holder after placing in it the sputtered sample with its underlying golden SEM

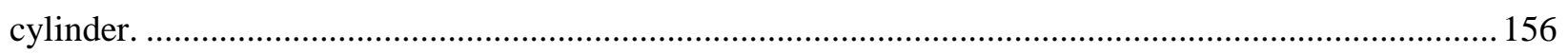

Figure D-1. The Minilock-Phantom III ICP-RIE system and the Network Tuning Controller................ 157 


\section{LIST OF TABLES}

Table 3.1. Summarized modeling results for the lattice of a square lattice of toluene holes in silicon .......44

Table 3.2. Summarized modeling results for the lattice of silicon pillars in toluene................................ 45

Table 3.3. Summarized simulation results of the bandgap for both polarizations of a triangular lattice of

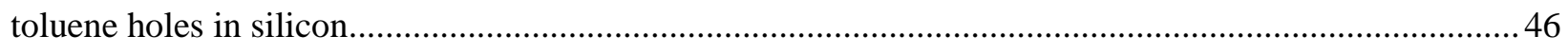

Table 3.4. Summarized simulation results of the bandgap for both polarizations of a triangular lattice of

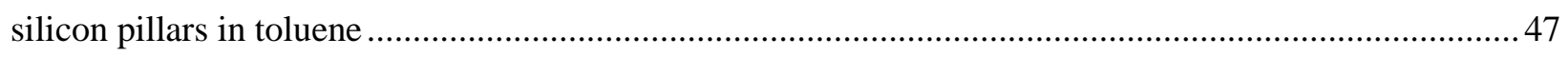

Table 3.5. Actual lattice parameters for a square lattice of infinitely-high silicon pillars surrounded by

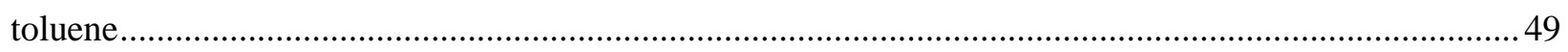

Table 3.6. Actual lattice parameters for a triangular lattice of infinitely-thick toluene holes in silicon..... 49

Table 3.7. Actual lattice parameters for a triangular lattice of silicon pillars surrounded by toluene ........ 49

Table 3.8. Table summarizing the bandgap measurement results for TE-like modes of a slab triangular lattice of toluene holes in silicon (sandwiched by air). $\mathrm{r} / \mathrm{a}=0.35 \mathrm{a}$.

Table 3.9. Table summarizing the bandgap measurement results for TE-like modes of a slab triangular lattice of toluene holes in silicon (sandwiched by air). $\mathrm{r} / \mathrm{a}=0.35$ .60

Table 3.10. Dimensions of a triangular lattice of toluene holes in silicon and sandwiched by air ..... 61

Table 3.11. Actual lattice parameters of a triangular lattice of air holes in PDMS ................................. 71

Table 3.12. Actual lattice parameters of a triangular lattice of PDMS pillars surrounded by air ...............71

Table 3.13. Actual lattice parameters of a triangular lattice of air holes in epoxy ....................................77

Table 4.1. The as-designed vs. as fabricated dimensions of the $\mathrm{PhC}$ in silicon. A deviation of only $2.84 \%$ was observed

Table 4. 2. The as-designed vs. as fabricated dimensions of the PhC in silicon. A deviation of only $0.87 \%$ was observed

Table 4.3. The actual dimensions of the diameter of the mater Si pillars and of the holes of the 5 molded PDMS PhC structures

Table 4.4. The actual dimensions of the diameter of the mater Si pillars and of the pillars of the 5 molded epoxy PhC structures 115

Table 5.1. Characteristics of the IR-emitting QDs that were used in the optical characterization experiment of the $\mathrm{PhCs}$ 118 


\section{CHAPTER 1: PHOTONIC CRYSTAL SENSORS}

\subsection{PHOTONIC CRYSTALS AND THEIR APPLICATIONS}

Controlling light propagation through a material has been the topic of intensive research since the pioneering works of E. Yablonoivitch [1] and S. John [2]. The objective at that time was to inhibit spontaneous emission and study light localization in periodic dielectric structures. The remarkable work of those two scientists marked the beginning of applying solid state physics theories and concepts to optics and electromagnetism. It was very soon realized that as the periodicity of solid state crystals determines the conduction and energy properties of their materials; the periodic dielectric structures that occur at scales comparable to the propagating wavelengths determine the transmission capabilities of photons through these materials [3-6].

Photonic crystal $(\mathrm{PhC})$ has been the title given to this new class of materials that is characterized by a periodically varying dielectric function $\varepsilon$. This periodicity can occur in one, two, or all orthogonal directions as shown in Figure 1.1. A typical example of the one-dimensional (1D) photonic crystals is the distributed Bragg reflector (DBR), in which layers of two or more different optical materials are stacked on top of each other in a periodic fashion. Although these DBRs are now considered a 1D class of PhCs, they were studied for several years before the findings of Yablonoivitch and John [7-10] and their unique ability to allow transmission of specific wavelengths while reflecting others was found to be dependent on the refractive index contrast between the layers as well as the thickness of each layer [7-10]. Currently, these structures are employed in a wide variety of optoelectronic devices [3]. Two-dimensional (2D) $\mathrm{PhCs}$ occur when the dielectric variation is engineered to be periodic in two directions and maintained constant along the third direction. This is achieved by either introducing holes of a low dielectric material with specific symmetry in a higher dielectric material, or simply the opposite case in which pillars of a high dielectric material are surrounded by a low dielectric background. In three-dimensional (3D) PhCs, the refractive index is varied along all the three directions of space. This is usually achieved using a bottom-up approach in which $1 \mathrm{D}$ or 2D PhCs slabs are carefully aligned and stacked to create a periodic variation along the third dimension [11].

The main interest in $\mathrm{PhCs}$ comes from their unique and very selective ability to control light propagating through them. As the electronic potential periodicity in a crystal determines the existence of the electronic band structure, the periodic dielectric potential of the PhCs allows the existence of allowed and forbidden bands (photonic bandgaps). Therefore, as will be discussed in Chapter 2, concepts such as: Block theorem, Brillouin zones, energy bands, dispersion relationships, etc. that were previously to study 


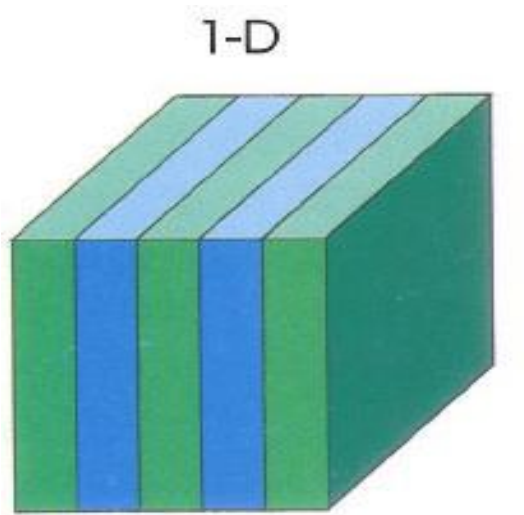

periodic in one direction

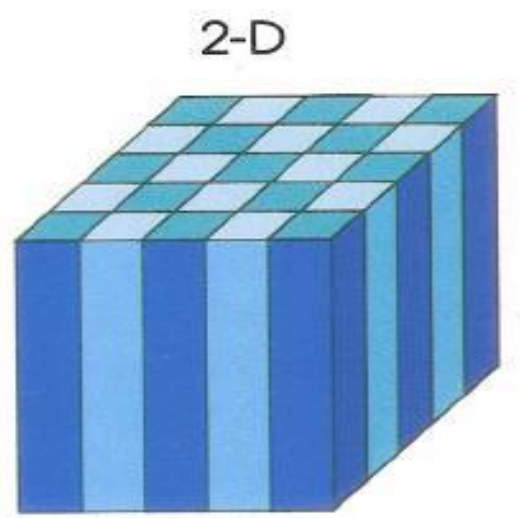

periodic in two directions

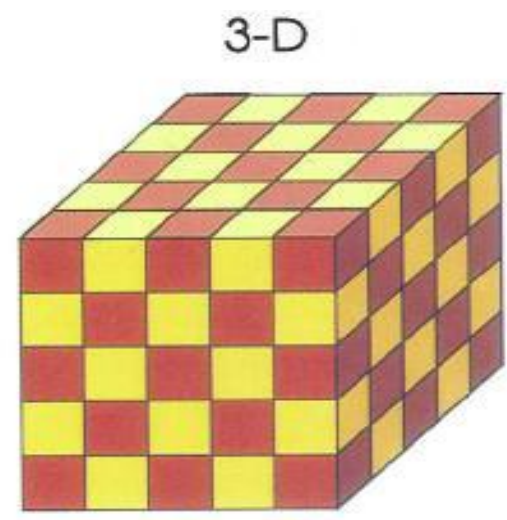

periodic in three directions

Figure 1.1. The three types of photonic crystals (PhCs) [6]

the interaction between electrons and their periodic lattices, were also utilized to understand photon motion within the periodic PhCs. This motion is governed by Maxwell's equations.

As light becomes incident on a PhC lattice of any dimensionality, it gets reflected off each layer of periodic media, and when suitable conditions are met, i.e. the periodicity is comparable in scale to the propagating wavelength and there is a sufficient refractive index contrast between the low- and high- $\varepsilon$ regions, light will no longer be allowed to propagate and a complete control over its leakage is possible. This unique feature of nanoscale $\mathrm{PhC}$ lattices has made them very attractive for a wide variety of applications such as waveguides and optical filters [3]. The more interesting attribute of $\mathrm{PhCs}$ is the ability to create localized modes by introducing defects in an otherwise ordered $\mathrm{PhC}$ lattice. Efficient resonators, beam splitters, lasers, and light emitting diodes (LEDs) are among the most common applications of PhCs [3]. A main objective of nanophotonic device research is to integrate all of these $\mathrm{PhC}$-based optical components on a single chip and be able to process optical signals in a similar fashion to how conventional electronic chips control electron flow.

In addition to the objective of creating integrated optical circuits, PhC lattices have been also the focus of intensive research involving optical-based sensors [12]. They have become the new nanotechnology-based class of optical sensors that have potential to provide ultra-sensitive detection capabilities that exceed that of the other sensor types: mechanical and electrochemical. In general, the demand for accurate sensors that can detect trace changes of the desired target has increased. With a wide variety of applications in the industrial and biomedical fields, this demand requires simple, fast, safe, portable, and inexpensive sensing platforms. It is also very important to produce reliable technologies that allow the testing of a larger number of samples in a timely manner. 
When altering any of the critical PhC parameters, such as: lattice constant, periodicity, or the materials of the high- and low- $\varepsilon$ regions, the range of allowed, blocked, and confined wavelengths is also altered. This has provided researchers with a very accurate and sensitive tool indicating not only the concentration or mass of a desired target, but also its specific type due to its refractive index signature. Two main detection modalities utilizing PhCs as the core structures can be realized: active and passive. Throughout the following sections of this chapter, a literature review is presented to explain the unique properties of each modality.

\subsection{THE ACTIVE DETECTION MODALITY UTILIZING PHOTONIC CRYSTALS}

Several critical parameters play a role in determining the optical bandgap properties of PhCs. If we are to take a simple 2D PhC lattice of a triangular symmetry of low- $\varepsilon$ holes in a high- $\varepsilon$ slab, as shown in Figure 1.2, then the following list demonstrates those parameters:

1. The dielectric constant of the slab $\left(\varepsilon_{1}\right)$

2. The dielectric constant of the hole $\left(\varepsilon_{1}\right)$

3. The slab thickness (h)

4. The lattice constant (a)

5. The radius of the holes ( $r)$

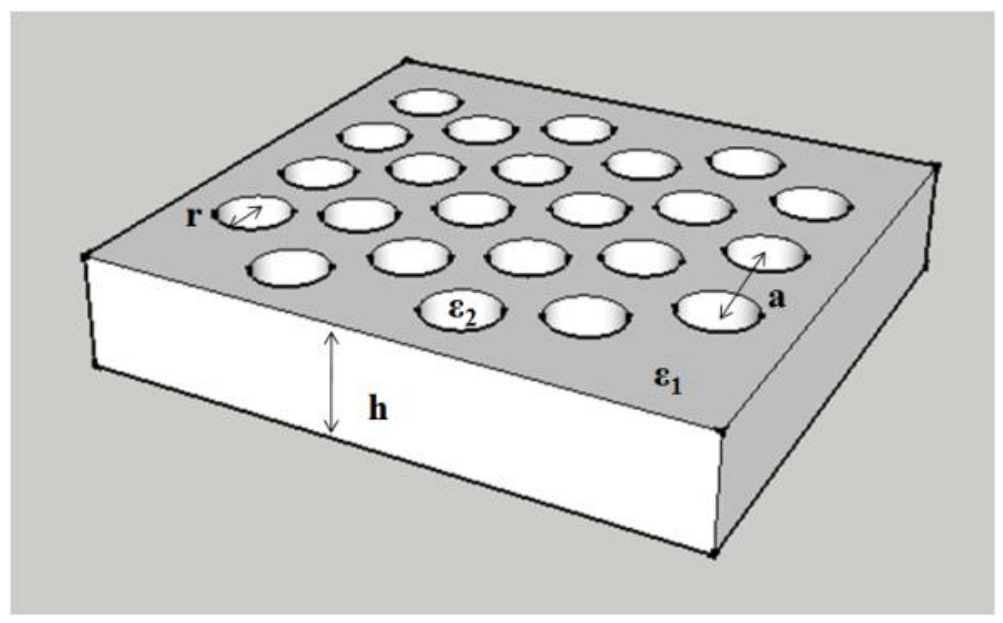

Figure 1.2. Critical parameters of a $2 \mathrm{D} \mathrm{PhC} \mathrm{slab}$ 
In the active detection modality, one of these parameters is intentionally modified when target analytes are introduced to the sensor with a specific $\mathrm{PhC}$ lattice. This translates into either changes of the radius and/or the lattice constant due to swelling effects, or changes of the effective refractive index as the target analytes replace the low- $\varepsilon$ regions of the $\mathrm{PhC}$ lattice. In most detection schemes utilizing this modality, a control sample is first tested to acquire initial information regarding the specific range of transmitted/reflected wavelengths. As the desired target is introduced, the change in one or more parameters translates into changes of the transmitted/reflected wavelengths. These changes are usually observed as red or blue shifts which are directly dependent on the local density of the adsorbed molecules and are very sensitive to the target type and quantity.

Chemical, humidity, gas, and protein biosensors are among the most common sensor types that utilize this active detection modality.

\subsubsection{Chemical Sensors}

Polymerized crystalline colloidal arrays (PCCAs) are self-assembled three-dimensional photonic crystal lattices that result from the electrostatic repulsion between highly charged spheres [13]. The repulsion allows the spheres to align in either a face-centered cubic (FCC) or a body-centered cubic (BCC) arrays and can very intensely Bragg diffract visible wavelengths. The unique characteristic of these arrays is their target-induced volume changes that cause the diffracted light to shift accordingly. For example, PCCA has demonstrated a unique ability to sense $\mathrm{pH}$ changes and the ionic strength of a specific chemical solution. By relating the diffracted wavelength to the lattice constant and the hydrogel volume, precise monitoring of the changes in $\mathrm{pH}$ and ionic strength is possible (Figure 1.3) [13]. Moreover, the same three dimensional photonic-crystal arrays, and based on volume-induced changes, were used develop a new sensing motif for the detection and quantification of creatinine, which is an important small molecule marker of renal dysfunction [14].

In a similar method, the three-dimensional crystalline colloidal arrays embedded within a polyacrylamide-poly(ethylene glycol) (PEG) hydrogel have demonstrated the ability to generate a colorimetric glucose recognition sensor [15]. As glucose of different concentrations is introduced to the array, it self-assembles the boronic acid and PEG functional groups into a supramolecular complex capable of introducing blue shifts to the photonic-crystal-based diffracted wavelengths. The visually evident diffraction color shifts across the visible spectral region using this method, from red to blue over physiologically important glucose concentration ranges. Thus, a new noninvasive glucose recognition is made possible [15-17]. 


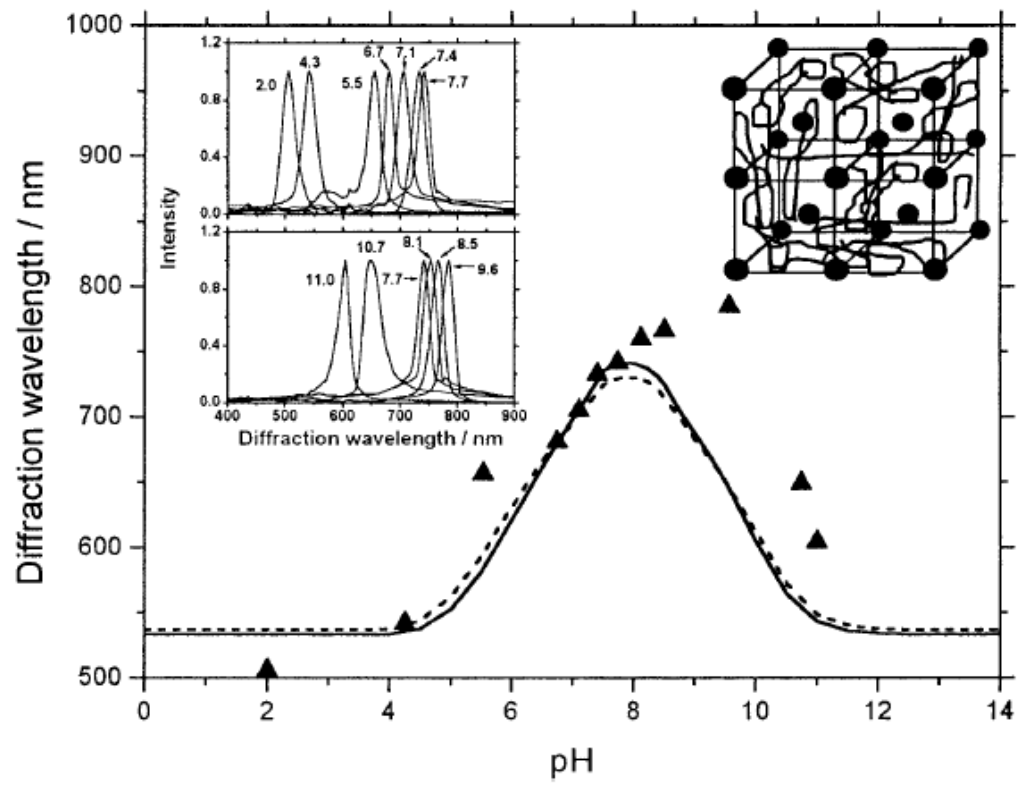

Figure 1.3. An example of a chemical sensor for $\mathrm{pH}$ changes in a solution. As the volume of the 3D polymer-based $\mathrm{PhC}$ changes, red or blue shifts can be observed accordingly [13]

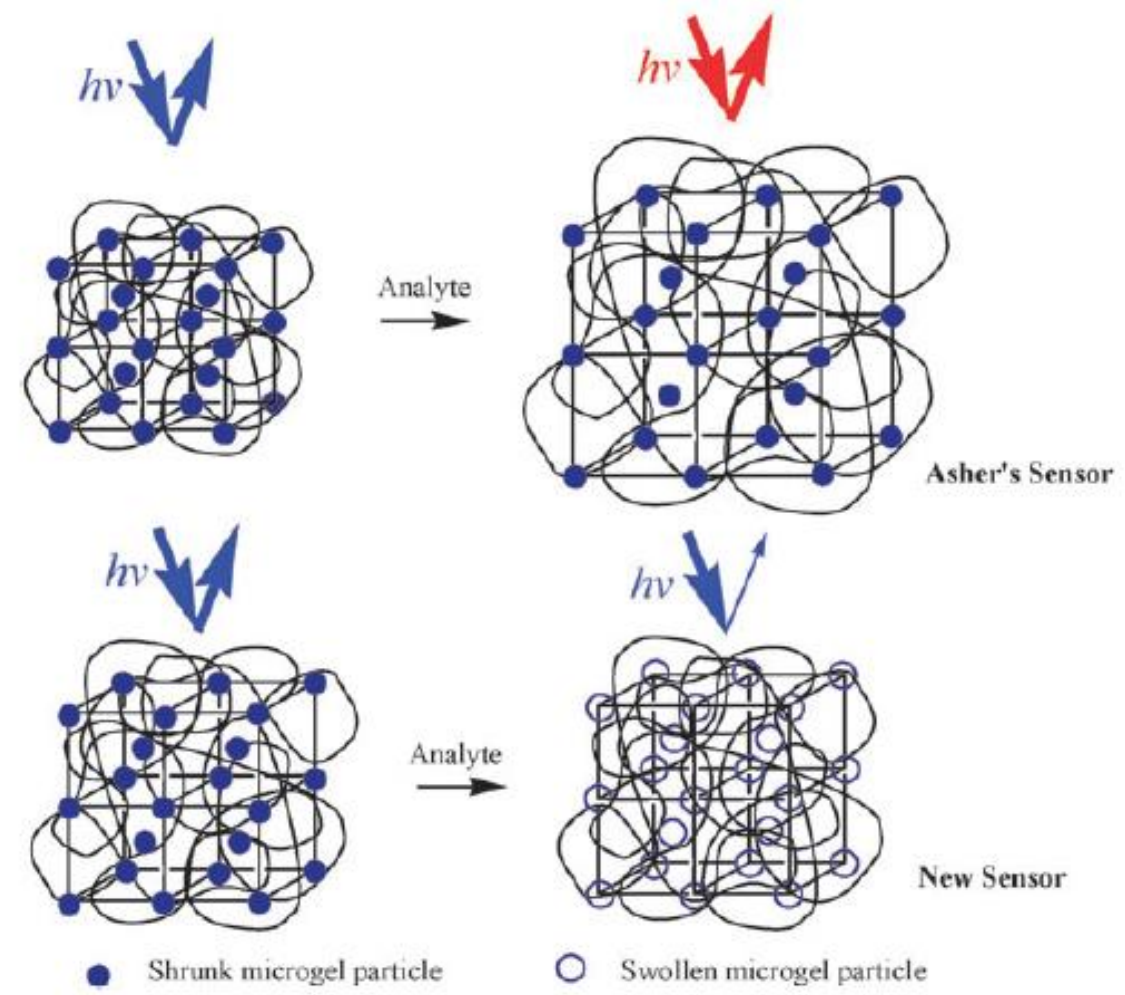

Figure 1.4. Examples of two 3D PhC based glucose sensing mechanisms. (Top) Changing lattice constant due to volume-induced changes. (Bottom) changing the radius due to swollen microgel particles chemical sensor for glucose using the three-dimensional PCCAs [17] 


\subsubsection{Humidity Sensors}

Hydrogel-based PhCs have demonstrated a unique ability to detect humidity changes with response times on the order of few seconds $[18,19]$. The ability of the PhC hydrogel structures to have this useful response to humidity is due to the acrylamide that makes up the polymer which possesses an excellent hydrophilicity and water absorption characteristics. As ambient humidity increases, the hydrophilic acrylamide hydrogel absorbs water from the air to reach a new equilibrium. This water absorption induces a deformation from its previous equilibrium contracted structure by attempting to swell in all directions. The swelling causes a spacing increase between the layers of the 3D PhC, and hence an increase in the lattice constant, which causes a shift of the reflectance spectrum peak. At normal incidence, strong interference of reflected light from the regular spaced alternating layers cause Bragg reflection at a specific wavelength. As the distance increases between the layers, a red-shift in the reflection peak is observed within few seconds (Figure 1.8) [18].

Other kinds of photonic crystal humidity sensors use nano-porous polymer photonic crystals [20]. As the humidity changes, the transmittance at the stop gap wavelength shifts due to changes in the refractive index of the structure. For example, a humidity percentage change from 40 to $90 \%$ is capable of inducing shifts of $43 \mathrm{~nm}$ in the stop gap position and resulting with an increase in the transmittance from $12 \%$ to $87 \%$ at $600 \mathrm{~nm}[20]$.
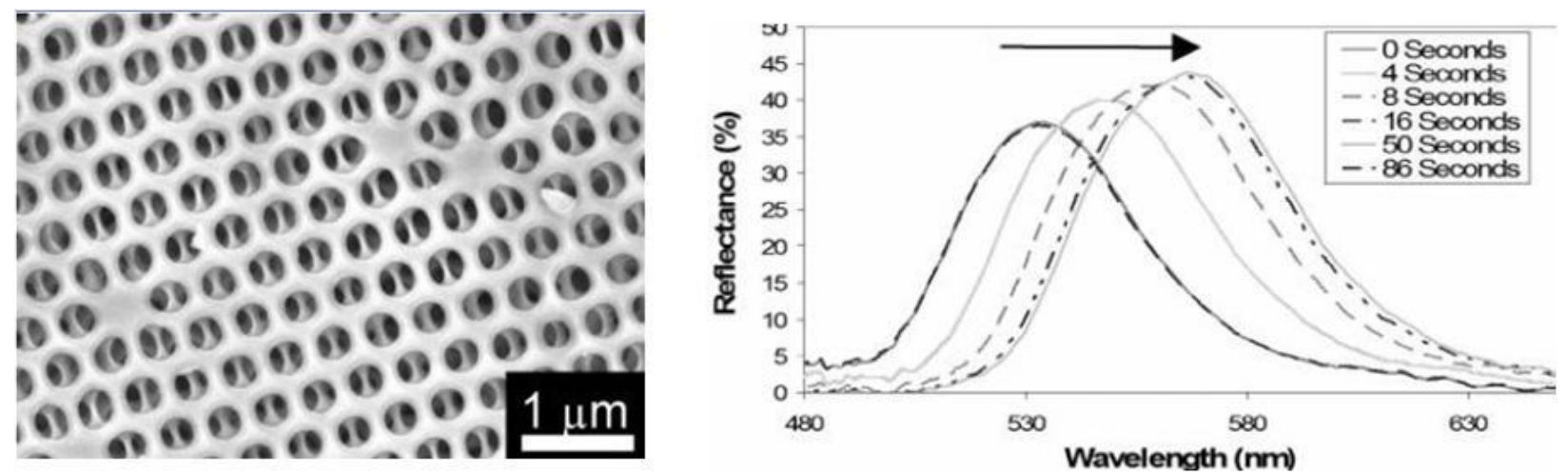

Figure 1.5. (Left) SEM image of 3D PhC-based hydrogel humidity sensor. (Right) Spectra shift with time from ambient conditions $50 \%$ to $80 \%$ RH [18].

\subsubsection{Gas Sensors}

The response of a 2D PhC resonator to changes of the ambient gas demonstrates another example of how photonic crystal lattices can be actively utilized in ultra-sensitive sensing platforms. For example, when a two-dimensional triangular PhC lattice fabricated in GaAs (Figure 1.7(a)) [21] with a resonant 
cavity was introduced to different gases, the refractive index within the holes surrounding the cavity changes accordingly. Such refractive index changes of the surrounding gas influence the effective refractive index of the membrane waveguide and the photonic band structure due to the change of the index contrast between the semiconductor and the holes. Both effects lead to a shift of the cavity resonance into the same direction [21]. In Figure 1.6, different transmission peaks are observed at the presence of three different ambient gases. A shift of the resonance toward higher wavelengths can be clearly observed as the refractive index of the ambient is increased from that of vacuum to Nitrogen and finally to $\mathrm{SF}_{6}$.

Moreover, air-guiding Photonic Bandgap Fibers (PBFs) have demonstrated the ability to sense both strongly (acetylene/hydrogen cyanide) and weakly (methane/ammonia) absorbing gases [22]. PBFs are very advantageous as gas sensors due to the long optical path that they introduce which allows more interaction between the gas and light mode field and therefore only very small sample volume is needed. Furthermore, PBFs can be bent and connected to standard fiber-optic instruments using advanced splicing techniques [22].
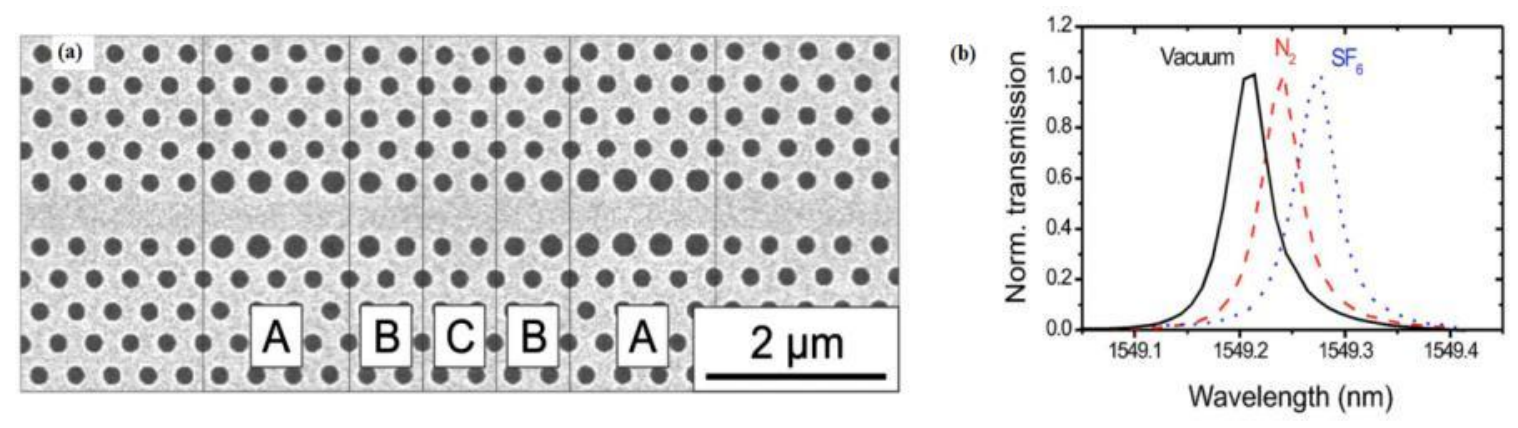

Figure 1.6. (a) Scanning electron microscopy image of the cavity (b) Transmission of a PhC resonator in vacuum (solid black line), nitrogen (dashed red line), and SF6 (blue dotted line) [21]

\subsubsection{Biosensor for Protein Detection}

A 2D PhC slab of a triangular array of air holes in silicon can also be used to analyze protein binding on the pore walls and quantitatively measure the protein diameter. In this unique and ultra-sensitive biosensor, a partial TE bandgap was realized in the triangular lattice with an r/a ratio of approximately 0.29. Since the PhC slab lacked a TM bandgap, all experiments were carried out using a TE polarized light at around $1.58 \mu \mathrm{m}[23-25]$.

Figure 1.7 illustrates the bio-molecule binding mechanism. Antibodies are first immobilized on the internal surface where they form a monolayer and capture the target protein molecules. When a probefunctionalized sensor is exposed to the target, a monolayer of target species is again captured on the 
surface of the sensor. Since the pore size of the $\mathrm{PhC}$ holes was 30 times larger than the protein hydrodynamic diameter, a uniformly thick monolayer of the proteins coated the pore wall. This biomolecule coating causes a refractive index change only in the vicinity of the pore wall. When proteins of different sizes coated the internal walls, different amounts of resonance red shift (Figure 1.8) were observed and very low masses of approximately 2.5 femtograms were detected. The device performance was verified by measuring the red shift corresponding to the binding of glutaraldehyde and bovine serum albumin (BSA) [23].

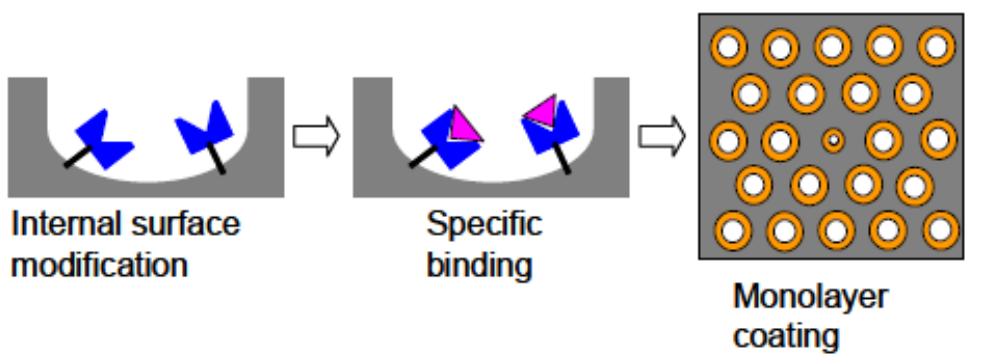

(a)

(b)

Figure 1.7. Schematic of bio-molecule recognition: (a) the target molecules are captured by the probe molecules. (b) The bio-molecules form a uniform layer on the internal surface of the sensor. In reality the layer thickness is very small compared with the pore size. [23]

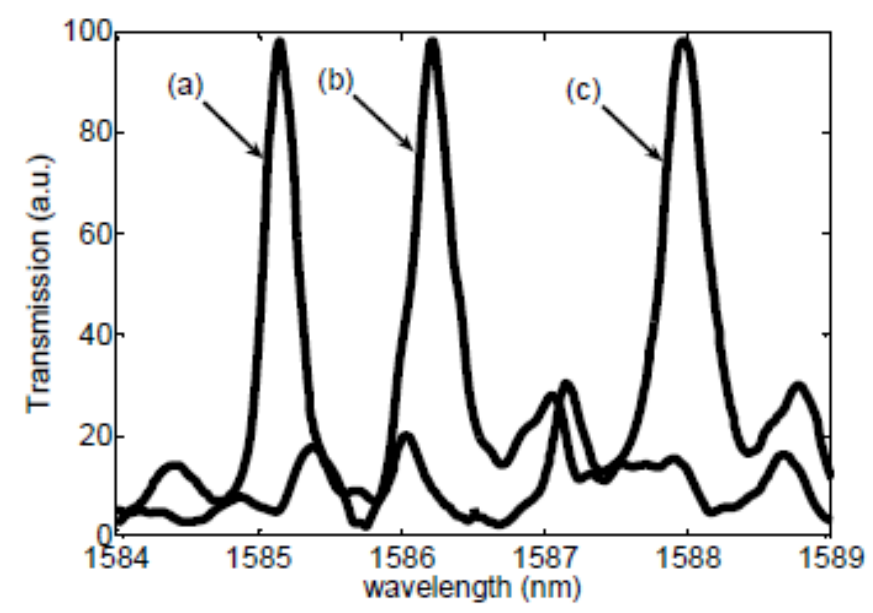

Figure 1.8. Normalized transmission spectra of the PC microcavity. Curve (a) indicates the initial spectrum resonance after oxidation and silanization, curve (b) is measured after glutaraldehyde attaches to the pore walls, and curve (c) is obtained after infiltration of BSA molecules. [23]

\subsubsection{Biosensor for Protein-DNA Interaction}

Understanding protein-DNA interactions is very important for the understanding of several cellular processes. The study of such an interaction has been dependent on small molecule disruptors. However, there are very few methods available for the rapid identification of compounds that disrupt protein-DNA interactions [26]. 
It was realized that the adsorption of these molecules on the surface of a $\mathrm{PhC}$ transducer proved to be a very effective method of allowing such an interaction to be examined with a variety of protein types. The PhC biosensor in this example is very sensitive to biomolecular interactions that occur on its surface. It is composed of a 1D periodic arrangement of dielectric material that very effectively prevents propagation of very narrow bands of light whose wavelength is directly dependent on the local density of the adsorbed biomolecules (Figure 1.9). Two very different protein-DNA interactions were successfully analyzed using this method; the bacterial MazEF complex, which binds to its promoter DNA in a sequence-specific manner, and the human AIF, a protein that binds nonspecifically to chromosomal DNA [26]. The sensor system utilizing these structures was capable of identifying compounds that prevent protein-DNA binding. The group predicted that such generality and simplicity of the PhC method should enable such structures to find broad utility for identification of compounds that inhibit protein-DNA binding [26].

a
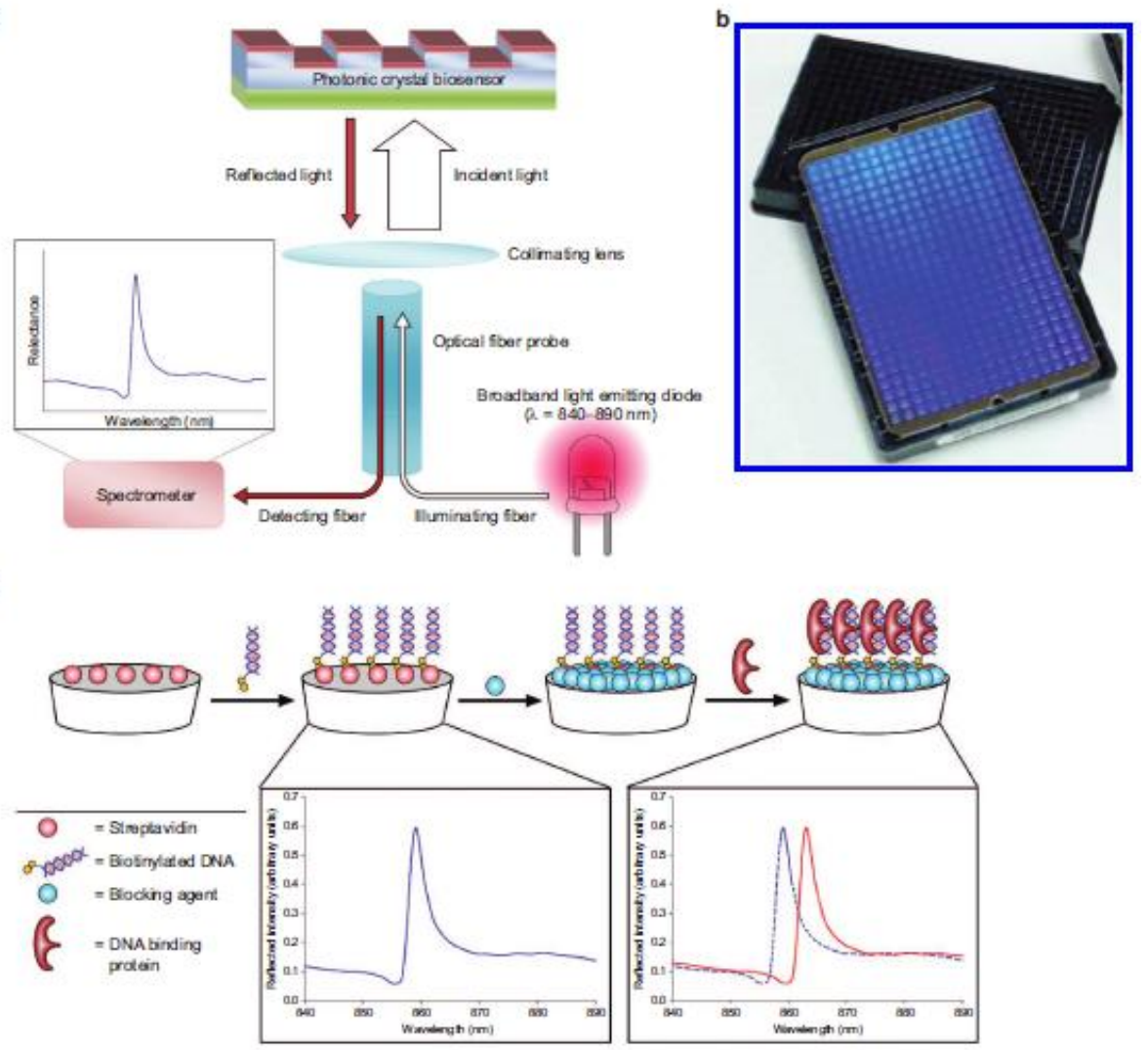

Figure 1.9. (a) Schematic of the PC biosensor. A broadband LED illuminates the biosensor from the bottom, and reflected light is collected and transferred to a spectrometer where the PWV is measured. (b) Image of PC biosensor films adhered to the bottom of black 384-well plates. (c) Diagram of protein-DNA binding experiments performed with PC biosensors. Streptavidin-coated biosensors are used to bind biotinylated DNA oligomers, and a distinct peak wavelength of the reflected light is observed. After the addition of Starting Block (Pierce Biotechnologies), a DNAbinding protein is added, and a shift in the wavelength of reflected light is observed [26] 


\subsection{THE PASSIVE DETECTION MODALITY UTILIZING PHOTONIC CRYSTALS}

In the active modality, as was explained previously, the introduction of a specific target to the system incorporating $\mathrm{PhC}$ lattices causes a critical change in one or more of the $\mathrm{PhC}$ parameters which then is translated into red or blue shifts of the transmitted/reflected incident light. The concept for passive detection utilizing $\mathrm{PhCs}$ is different in a sense that all parameters are designed to remain essentially constant throughout the whole detection experiment, and hence the PhC lattice acts as efficient light manipulative tool that controls and guides the emission from labeled molecules toward a specific direction. Therefore, it is fair to say that the PhC lattices alone are not sensors. They are simply passive elements within their sensors that efficiently manipulate and guide light to propagate toward conventional detectors that analyze the produced signal.

This modality is mostly utilized for biosensing applications. However, it is much less common than the active one. The reason is due to the increased interest in developing biosensors that do not involve an extra labeling step that may alter the desired target and not allow it to be reused. On the other hand, for several other applications, rapid DNA detection for molecular biometrics for instance, laser induced fluorescence (LIF) is considered the fastest and most common method to detect labeled or naturally fluorescing biomolecules at very low concentrations [27-29]. Modern bench-top fluorescence analysis instruments are capable of generating accurate quantitative measurements of specific target analytes, leading to widespread use in biomedical and environmental applications. Fluorescence spectroscopy offers excellent sensitivity and low detection limits for a wide range of fluorescent label/molecule combinations. However, the bench-top nature of diagnostic systems that employ these methods does not lend itself well to sample analysis outside of dedicated laboratories in point-of-use or point-of-care scenarios. The following two sub-sections will explain two common detection methods in which $\mathrm{PhC}$ lattices with specific photonic bandgaps are utilized to cause at least 27-fold enhancement of the fluorescence signal from labeled molecules on the surface of the $\mathrm{PhC}$ or within high quality-factor resonant cavities.

\subsubsection{Surface Fluorescence Enhancement Biosensor}

Labeling target analytes for visualization and quantification purposes is very important to life science procedures that involve imaging of cells and their components as well as gene expression profiling. Research on the effects of metals on enhancing fluorescence and specifically that of surface plasmon resonance has demonstrated promising results [30-35]. However, it was soon observed that such fluorescence enhancement on metal surfaces can also undergo non-uniform enhancement when colloidal 
nanoparticles serve as the substrate. Patterned metal-based nanostructures have been pursued in hopes to resolve this issue, but the large-area fabrication of such nanostructures has been a limiting factor of such methods. Moreover, fluorophore quenching of labeled molecules that are in the vicinity of the metal substrates $(\sim 20 \mathrm{~nm})$ was another factor that limited these methods from being employed to large-scale enhancement sensors [36].

A proposed efficient solution to the previous limitations is through the use $\mathrm{PhCs}$ which have demonstrated capabilities of fluorescence enhancement through mechanisms similar to those observed with metal nanostructures. PhCs fabricated in dielectric materials proved to be very effective at reducing quenching effects of fluorophores close to the PhC surface [37]. After careful design, the dielectric-based PhCs allow for narrow-band reflections with spectral properties determined by the PC dimensions.

Since the narrow-band reflections of the proposed 1D PhC varies with position at a fixed illumination angle, a single device can potentially exhibit reflections at a range of wavelengths including that of fluorophore's excitation wavelength. To achieve this functionality, a thin high refractive index $\mathrm{TiO}_{2}$ layer was deposited on the dielectric-based 1D PhC (Figure 1.10). By coating the device with a monolayer of Cy5-conjugated protein and scanning it at normal incidence with a microarray scanner, a spatial map of enhanced fluorescence was generated in order to determine the reflected wavelength that corresponds to the greatest enhancement. The group reported $\mathrm{PhC}$ an 18-fold fluorescence enhancement when such a reflection overlaps the fluorophore excitation wavelengths [37].

In a recent work demonstrated by the same group [38], a one-dimensional PhC was used to experimentally demonstrate that the detection of fluorescent molecules on a $\mathrm{PhC}$ surface can be substantially enhanced through the combined effects of resonance-enhanced excitation of the fluorescent dye, resonance-enhanced extraction of the fluorescence emission and a dielectric nanorod surface coating increasing the surface area available for fluorophore-PC interaction [38]. Enhanced excitation is obtained by engineering a high-Q TM resonant mode to efficiently couple with an incident TM-polarized $\lambda=633$ $\mathrm{nm}$ laser for exciting Cyanine-5 (Cy5). Enhanced extraction results from a low-Q TE resonance designed to spectrally overlap the Cy5 emission spectrum for channeling TE-polarized emission towards the detection instrument. To increase the emission based on surface area, the entire surface is coated with $\mathrm{TiO}_{2}$ nanorods that allows more fluorophores to penetrate into the region of enhanced near-electric fields. Experimental results reveal a 588-fold enhancement in fluorescence intensity relative to an unpatterned glass surface. Figure 1.11 demonstrates the enhancement results as well as a schematic of the proposed one-dimensional photonic crystal structure [38]. 
(a)
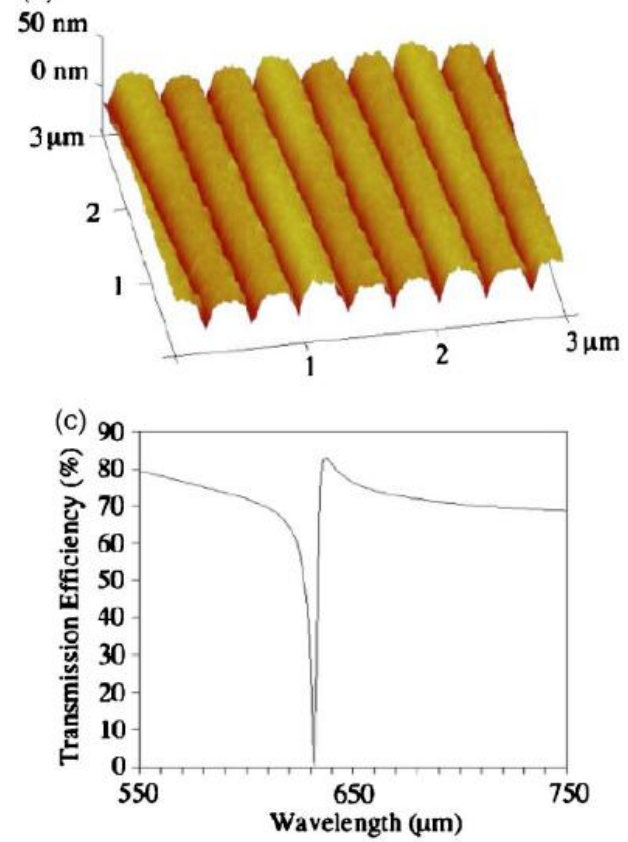

(b)
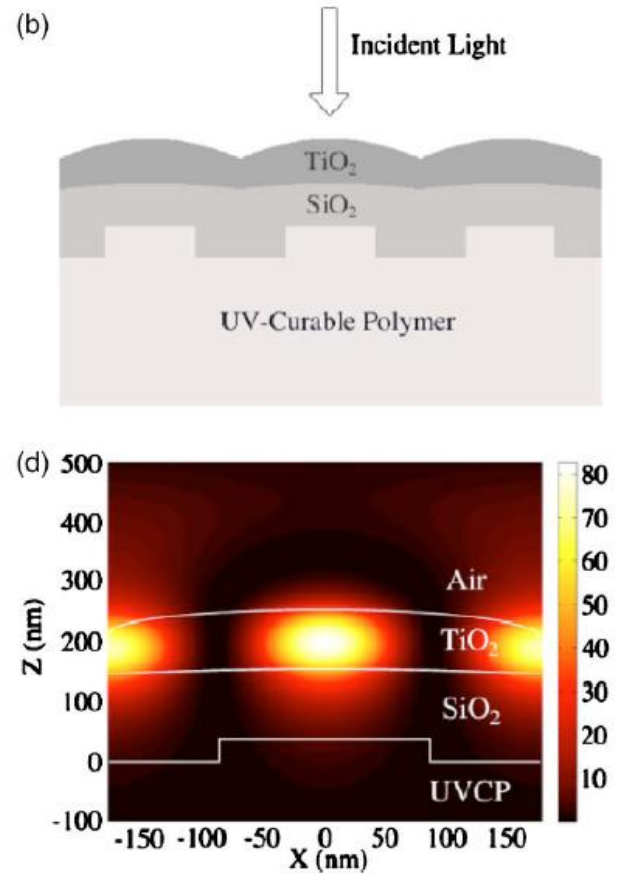

Figure 1.10. (a) Atomic force microscopy image of a portion of the photonic crystal surface. (b) Side view of the model used in Rigorous Coupled-Wave Analysis computations of the structure response. (c) Simulated transmission response as a function of wavelength under TE illumination for a region of the structure resonant at $633 \mathrm{~nm}$. (d) Simulated electric field intensity for the structure in (b) when illuminated with $633 \mathrm{~nm}$ TE-polarized light at normal incidence. [37]
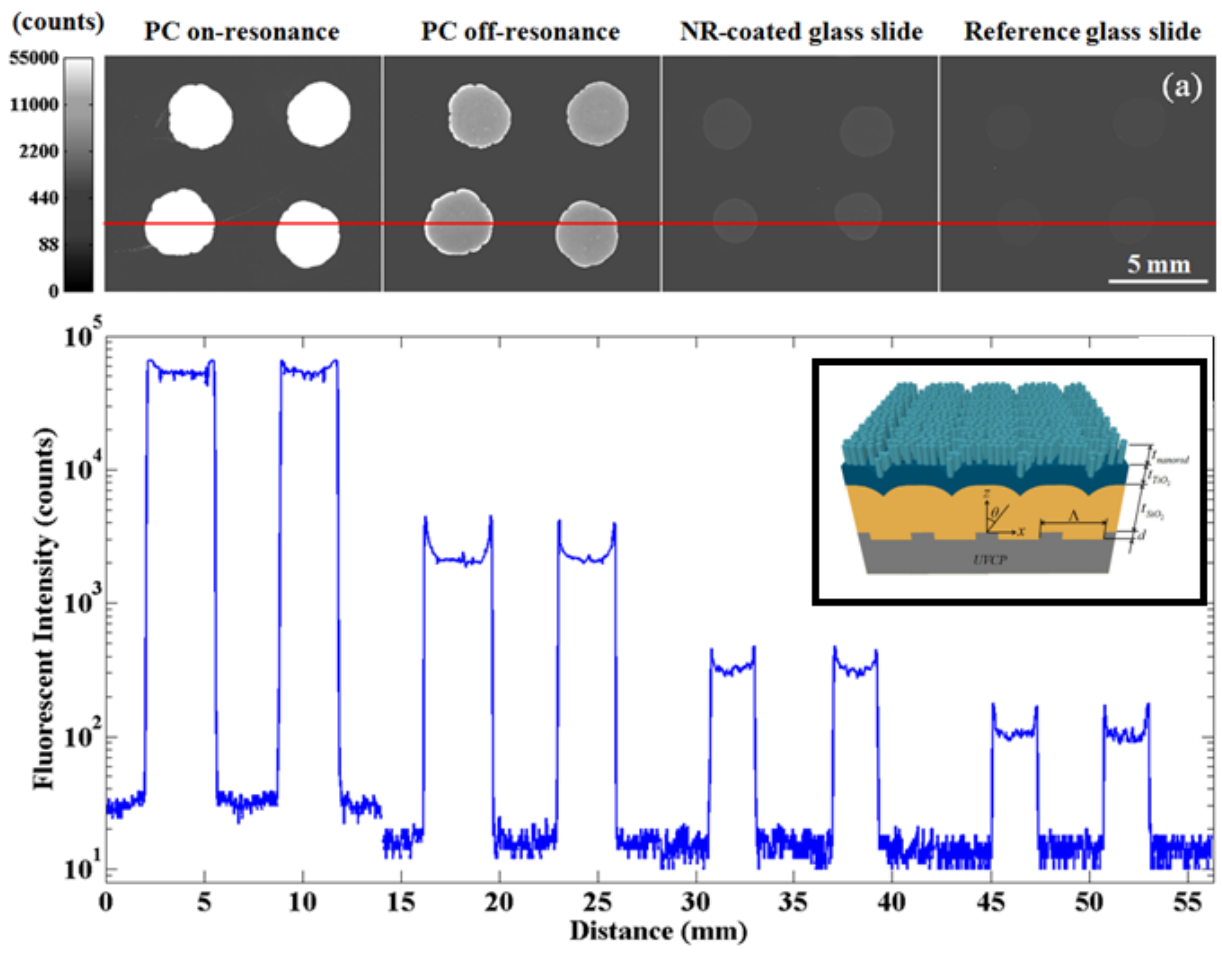

Figure 1.11. Fluorescence enhancement on the surface of a 1D PhC with nano-rods as shown in image. inset [38] 


\subsubsection{Resonant-Cavity-Based Fluorescence Enhancement Biosensor}

In the PhC-based transduction mechanism developed by our group [39-46], the PhC structure is considered a passive element that is utilized as a slab waveguide enhancing emission from labeled molecules that fall within its bandgap. The enhancement results from the fact that frequencies that fall within the bandgap of a two-dimensional PhC slab are not allowed to leak outside of the slab if the slab contained at least 14 periods of varying dielectric constant regions. Therefore, a two-dimensional $\mathrm{PhC}$ slab is designed to have a bandgap surrounding an emission of quantum dots (QDs) that emit around 1100 $\mathrm{nm}$. Exciting the quantum dots from within the $\mathrm{PhC}$ forces the emitted light to be confined within the $\mathrm{PhC}$ region and leak only vertically where a detector is placed. Using this method, 27-fold enhancement of the emitted light is experimentally recorded. Figure 1.12 summarizes this detection mechanism [46].

Achieving much higher enhancement factors is possible through the incorporation of resonant cavities within the PhC lattices. The resonant cavities confine the emitted wavelengths that originally fall within the bandgap into much smaller volumes. As the volume of the cavity is reduced, Purcell's effect can cause larger enhancement factors by increasing the spontaneous emission rate of the QDs within a PhC point defect nanocavity. This can lead to more photons per unit time and area, resulting in an enhancement in fluorescence that is proportional to the quality factor $(\mathrm{Q})$ of the defect.

\subsection{THESIS ORGANIZATION}

The work explained throughout the next four chapters of this thesis focuses on utilizing $\mathrm{PhC}$ lattices fabricated in thin-film semiconductor materials and polymers as passive elements of a fluorescenceenhancement-based biosensor. The detailed theory behind photonic crystals and their unique attributes will be explained in Chapter 2. Modeling results of several symmetric and asymmetric PhC lattices using frequency and time-domain simulation tools to extract the critical parameters will be demonstrated in Chapter 3. These parameters provide us with the dimensions of the desired lattice properties that result in light/lattice interaction properties that lead to proper device function. The fabrication processes that were developed to realize these lattices will be explained in Chapter 4. This also includes the novel soft-

lithographic techniques that were developed to create PDMS- and Epoxy- based PhCs. Chapter 5 discussed the characterization results of the fluorescence enhancement qualities of these lattices. Conclusions and future directions are summarized in Chapter 6. 

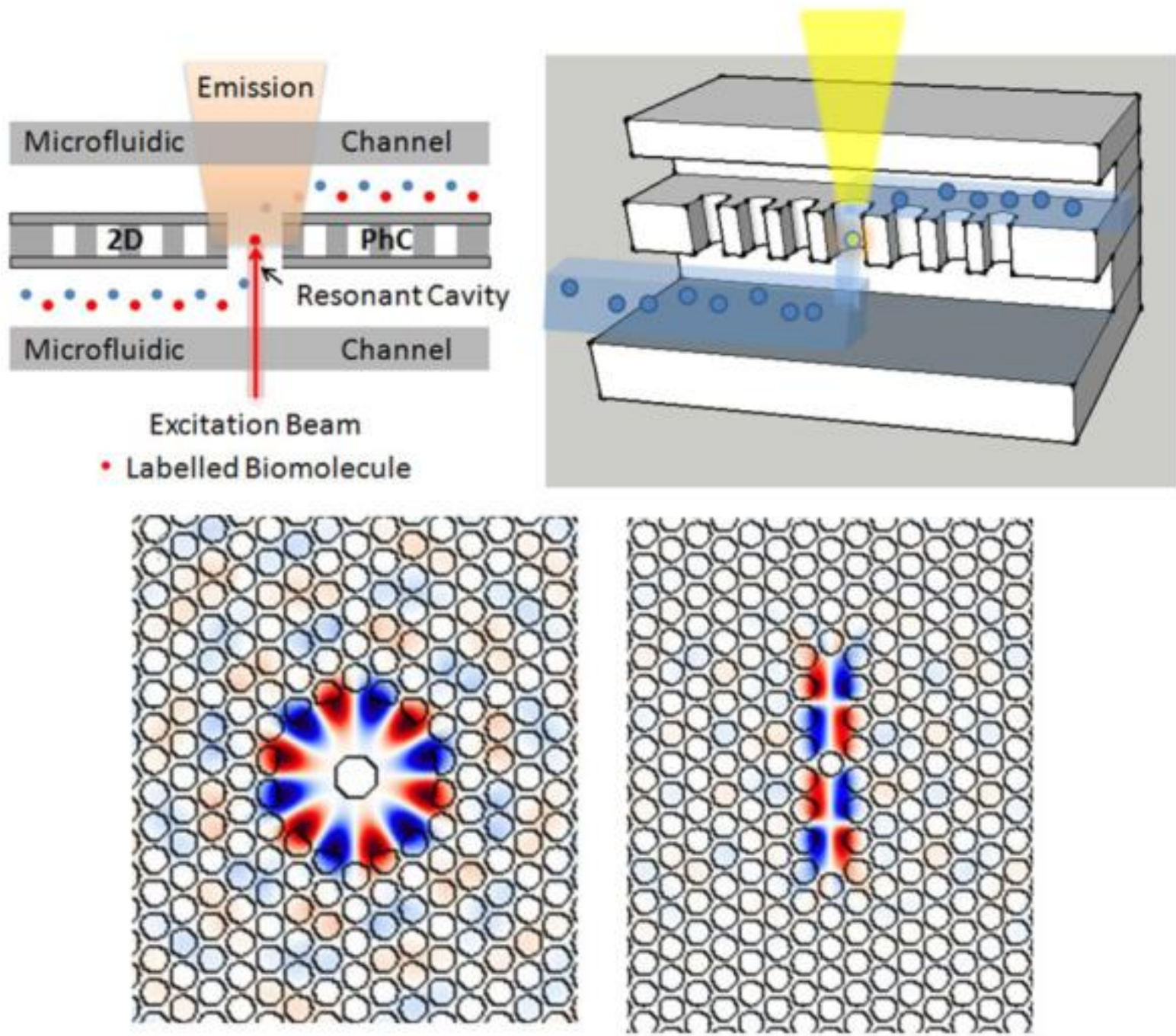

Figure 1.12.( Top) two and three dimensional representation of the fluorescence enhancement caused by the spatial confinement of the emitted light within the PhC. The resonant cavity is carefully designed to cause the emitted light from the labeled molecule to be trapped and leaked toward a detector above the PhC slab. (Bottom) Finite Difference

Time Domain (FDTD) [46] 


\section{CHAPTER 2: PHOTONIC CRYSTAL THEORY}

\subsection{PHOTONIC CRYSTALS' THEORY - LITERATURE REVIEW ${ }^{1}$}

A time varying electric field can create a magnetic field and a time varying magnetic field can produce an electric field. Such a behavior of the electric and magnetic fields are governed by a unique set of formulas known as Maxwell's equations. These equations are considered the rules that govern all classical electromagnetic phenomena which were put forth by James Clerk Maxwell [3-6].

To fully understand the behavior of electromagnetic waves in photonic bandgap materials, it is better to first discuss the similarities between the Schrodinger and Maxwell equations that govern the motion of electrons and photons, respectively. The electron behavior is described using the Schrodinger equation [36]

$\left[\frac{-\mathrm{h}^{2}}{8 \pi^{2} \mathrm{~m}^{*}} \nabla^{2}+\mathrm{V}(\boldsymbol{r})\right] \varphi(\mathbf{r})=\mathrm{E} \varphi(\mathbf{r})$ Equation 2.1

Where $\left[\frac{-h^{2}}{8 \pi^{2} \mathrm{~m}^{*}} \nabla^{2}+\mathrm{V}(\mathbf{r})\right]$ is called Hamiltonian operator, $h$ is Planck's constant, $\mathrm{m} *$ is the effective mass of the electron, $\mathrm{V}(\mathbf{r})$ is the potential function, $\mathrm{E}$ is the total energy, and $\varphi(\boldsymbol{r})$ is the wave function of the electron, which is interpreted as the probability amplitude function. For a defect-free crystal lattice, the potential is periodic; $\mathrm{V}(\mathbf{r})=\mathrm{V}(\mathbf{r}+\mathbf{R})$, where $\mathbf{R}$ is a vector that represents the displacement between plane waves that are modulated by an amplitude function relative to the periodicity of the lattice. These waves are commonly known as Bloch waves [3].

On the other hand, the electromagnetic fields are governed by classic electromagnetic theory. The fields are described using four equations that relate the electric field to the magnetic field and to other present sources or charges within the medium. These are called Maxwell's equations and in the absence of magnetic media, two of the four equations can be combined, as will be explained in the following section, to yield the final master photonic crystal equation (Equation 2.2).

$\left[\nabla \times \frac{1}{\varepsilon(\mathbf{r})} \nabla \times\right] \mathbf{H}(\boldsymbol{r})=\left(\frac{\omega}{c}\right)^{2} \mathbf{H}(\boldsymbol{r})$ Equation 2.2

\footnotetext{
${ }^{1}$ The derivation of the photonic crystal master equation demonstrated here is based on [3-6]. Therefore, detailed explanation of this derivation can be found in these references.
} 
Where $\left[\nabla \times \frac{1}{\varepsilon(r)} \nabla \times\right]$ is Maxwell's operator, $\mathbf{H}(\mathbf{r})$ is the magnetic field, $\omega$ is the angular frequency, $c$ is the speed of light in vacuum, and $\varepsilon(\mathbf{r})$ is the dielectric permittivity. Similar to the atomic crystalline lattice, photonic crystals have a periodic dielectric structure, $\varepsilon(\mathbf{r})=\varepsilon(\boldsymbol{r}+\boldsymbol{R})$, and solutions are also Bloch waves. It is obvious that both equations, 2.1 and 2.2, are of the eigenvalue/eigenfunction type. They both describe a wavelike function in space, $\varphi(\mathbf{r})$ in 2.1 and $\mathbf{H}(\boldsymbol{r})$ in 2.2, and each wave exists in a periodic medium characterized by a periodic potential V (for electrons) and a periodic permittivity $\varepsilon$ (for photons). The Hamiltonian operator in Schrodinger's equation and Maxwell's operator in the master PhC equation are both Hermitian operators; second-order space differential operators [3]. The corresponding real eigenvalues to these two equations are the electron energy $\mathrm{E}$ and the squared frequency of the electromagnetic wave $\omega^{2}$, respectively.

The coherent scattering at each lattice location in electronic and photonic lattices creates bands in the eigenvalue spectrum in which propagation is prohibited. Moreover, when defects are introduced in the periodic medium, by doping for instance, localized states are created with eigenvalues solutions that did not exist in the defect-free lattice. As the defect density increases, trapped waves will have higher chances of tunneling to nearby defect sites to permit the propagation of states with eigenvalues inside the bandgap of the bulk crystal. In semiconductor devices, this doping is very critical to the functionality of many devices such as: diodes, transistors, and light-emitting diodes (LEDs). In a similar fashion, introducing defects in periodic photonic crystal lattices has many applications such as: high-Q factor resonators and efficient waveguides [3]. It is then obvious that such common analogies between Schrodinger and Maxwell eigenvalue equations will encourage researchers to apply the useful solid-state physical phenomena such as: Bloch waves, reciprocal lattice, Brillouin zone, band diagram, dispersion diagrams, etc. to the periodic photonic crystal lattices and strive to produce similar solutions that could potentially result with a new class of optical devices.

However, the physical nature of the magnetic field is a vector quantity, while the electronic wave function is a scalar quantity. Moreover, Maxwell's equations posses no characteristic length scale which contradicts completely with the Schrodinger equation in which all of its physical constants, i.e. Planck's constant, the electron mass, and Bohr radius, are basic scales of length. Therefore, any scaling in the dimensions of an electronic crystal will have a significant impact on the expected outcome. On the other hand, the same scaling operation can be applied to a photonic problem and only the frequencies will be affected by a predetermined scaling factor. These distinctions arise from the fact that the atoms of semiconductor crystalline solids are naturally occurring while atoms of a $\mathrm{PhC}$ lattice are engineered and fabricated for a specific application which is a complex process, as will be discussed in Chapter 4[3-6]. Therefore, the scale invariance of Maxwell's equations was utilized to test the ability to fabricate lattices 
for microwave scales with confidence that the obtained unique optical properties will be maintained when moving to smaller-scale wavelengths and lattices upon the availability of fabrication tools or methods for this new scale. Moreover, Yablonovitch indicated that the band theory assumes uncorrelated wave functions of multiple particles. This fits the electromagnetic behavior very well as they obey the principle of superposition but for electrons, their strong interactions make such a theory inapplicable [3].

\section{Maxwell's Equations for Photonic Crystals}

To study the propagation of electromagnetic waves in photonic crystals, we begin with Maxwell equations. The four macroscopic Maxwell equations in SI units are [3-6]:
$\nabla . \mathbf{B}=0$ Equation 2.3
$\nabla . \mathbf{D}=4 \pi \rho$ ..Equation 2.4
$\nabla \times \mathbf{E}+\frac{1}{\mathrm{c}} \frac{\partial}{\partial \mathrm{t}} \mathbf{B}=0$ Equation 2.5
$\nabla \times \mathbf{H}+\frac{1}{\mathrm{c}} \frac{\partial}{\partial \mathrm{t}} \mathbf{D}=0$ Equation 2.6

Where $\mathbf{E}$ is the electric field, $\mathbf{B}$ is the magnetic field, $\mathbf{D}$ is the displacement field, $\mathbf{H}$ is the magnetic field strength, $\rho$ represents free charges, and $\mathbf{J}$ represents free currents. Since photonic crystals are normally fabricated in dielectric materials, no free charges or currents in the material makes the assumption $\rho=\mathrm{J}=0$ valid. Moreover, for most dielectric materials of interest with properties independent of direction and magnetic permeability, the relative magnetic permeability $\mu(\mathbf{r})$ is very close to unity and also for simplicity the magnetic field can be written as $\mathbf{B}=\mu_{o} \mathbf{H}$. With $\varepsilon$ being dielectric permittivity and is equal to the square of the refractive index $n$, we can relate the displacement field to the electric field as well as the magnetic field to the magnetic field strength as shown in Equations 2.7 and 2.8 [4]
$\mathbf{D}(\mathbf{r})=\varepsilon_{0} \varepsilon(\mathbf{r}) \mathbf{E}(\mathbf{r})$ Equation 2.7
$\mathbf{B}(\mathbf{r})=\mu_{0} \mu(\mathbf{r}) \mathbf{H}(\mathbf{r})$ Equation 2.8

Maxwell's Equations then can be re-written as follows:

$\nabla \cdot \mathbf{H}(\mathbf{r}, \mathrm{t})=0$ Equation 2.9

$\nabla \cdot[\varepsilon(\mathbf{r}) \mathbf{E}(\mathbf{r})]=0$ Equation 2.10

$\nabla \times \mathbf{E}(\mathbf{r}, \mathrm{t})+\mu_{0} \frac{\partial \mathbf{H}(\mathbf{r}, \mathrm{t})}{\partial \mathrm{t}}=0$ Equation 2.11 
$\nabla \times \mathbf{H}(\mathbf{r}, \mathrm{t})-\varepsilon_{0} \varepsilon(\mathbf{r}) \frac{\partial \mathbf{E}(\mathbf{r}, \mathrm{t})}{\partial \mathrm{t}}=0$

Equation 2.12

In general, both $\mathbf{E}$ and $\mathbf{H}$ are functions of time and space. However, due to the linearity of Maxwell equations, we can separate the time dependency from the spatial dependency by expanding the fields into a set of harmonic modes. Using Fourier analysis, the decomposition of a function into a number of sinusoidal functions of different frequencies can be done and then recombined to obtain the original function [4]. Therefore, $\mathbf{E}$ and $\mathbf{H}$ can be expanded into a set of harmonic modes that sinusoidally vary in time as follows:

$\mathbf{H}(\mathbf{r}, \mathrm{t})=\mathbf{H}(\mathbf{r}) \mathrm{e}^{-\mathrm{i} \omega \mathrm{t}}$ Equation 2.13

$\mathbf{E}(\mathbf{r}, \mathrm{t})=\mathbf{E}(\mathbf{r}) \mathrm{e}^{-\mathrm{i} \omega \mathrm{t}}$ Equation 2.14

To find the equations governing the mode profiles for a given frequency, we insert the above equations into Equations 2.9-2.12. The two divergence equations give the condition [4]:

$\nabla \cdot \mathbf{H}(\mathbf{r})=\nabla \cdot[\boldsymbol{\varepsilon}(\mathbf{r}) \mathbf{E}(\mathbf{r})]=0$ Equation 2.15

This means that no point sources or sinks of displacement and magnetic fields exist in the medium. The two curl equations relate $\mathbf{E}(\mathbf{r})$ to $\mathbf{H}(\mathbf{r})$

$\nabla \times \mathbf{E}(\mathbf{r})-\mathrm{i} \omega \mu_{\mathrm{o}} \mathbf{H}(\mathbf{r})=0$ Equation 2.16

$\nabla \times \mathbf{H}(\mathbf{r})+\mathrm{i} \omega \varepsilon_{0} \varepsilon(\mathbf{r}) \mathbf{E}(\mathbf{r})=0$ Equation 2.17

By dividing equation 2.17 by $\varepsilon(\mathbf{r})$ and then taking the curl, equation 2.16 then can be used to eliminate $\mathbf{E}(\mathbf{r})$. Moreover, the constants $\varepsilon_{\mathrm{o}}$ and $\mu_{\mathrm{o}}$ can be combined to yield vacuum speed of light [4],

$c=\frac{1}{\sqrt{\varepsilon_{\mathrm{o}} \mu_{\mathrm{o}}}}$ Equation 2.18

The result is:

$\left[\nabla \times\left(\frac{1}{\varepsilon(\mathbf{r})} \nabla \times \mathbf{H}(\mathbf{r})\right)=\left(\frac{\omega}{c}\right)^{2} \mathbf{H}(\mathbf{r})\right] \quad$ or, $\left[L \mathbf{H}(\boldsymbol{r})=\left(\frac{\omega}{\mathrm{c}}\right)^{2} \mathbf{H}(\mathbf{r})\right]$, where $L \mathbf{H}(\boldsymbol{r}) \equiv \nabla \times\left(\frac{1}{\varepsilon(\mathbf{r})} \nabla \times \mathbf{H}(\mathbf{r})\right)$ .Equation 2.19 
Equation 2.19 is called the master equation. $L$ represents a differential operator. The master equation can be solved first to find the modes $\mathbf{H}(\mathbf{r})$ and the corresponding frequencies then Equation 2.17 can be used to recover $\mathbf{E}(\mathbf{r})$ as follows:

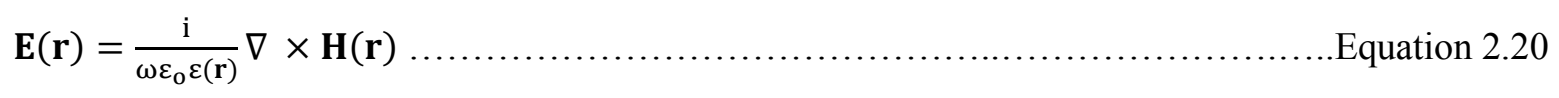

The reason why the problem was formulated in terms of $\mathbf{H}(\mathbf{r})$ and not $\mathbf{E}(\mathbf{r})$ is mainly due to mathematical convenience.

\subsection{THE PHOTONIC BANDGAP}

Photonic crystals have discrete translational symmetry that is not invariant under translations of any distance, but rather, only distances that are a multiple of some fixed step length which is commonly known as the lattice constant. The simplest example of such system is the 1D photonic crystal (Figure 2.1)

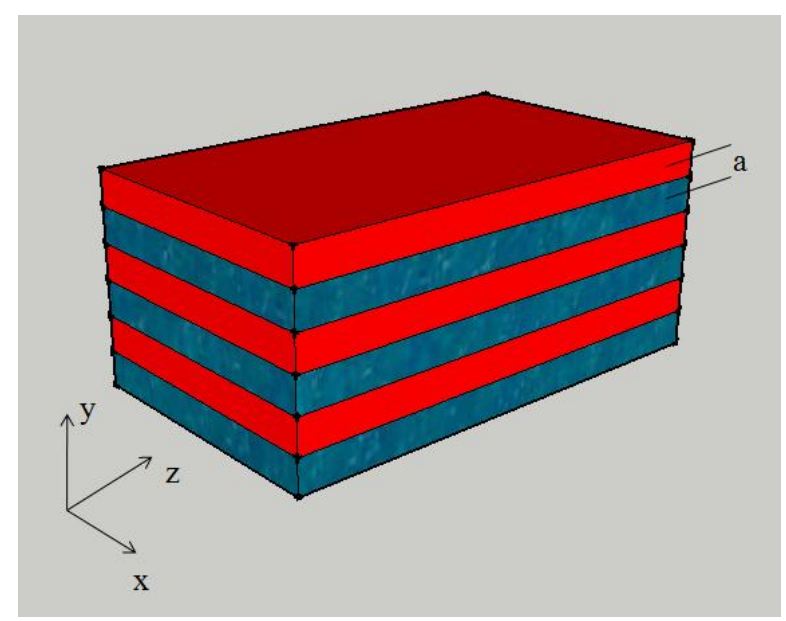

Figure 2.1. A 1D photonic crystal with varying dielectric constant in the $y$ direction. Red layers can represent high refractive index regions while blue layers can represent low refractive index regions

For this system, a continuous translational symmetry can be observed in the $\mathrm{x}$ and $\mathrm{z}$ directions and a discrete translational symmetry in the y direction with a basic step length which is called the lattice constant, $a$. In other words, the dielectric constant is only varying along the y direction. The discrete symmetry will allow us to write the dielectric function in terms of the lattice constant as follows [3-6],

$\varepsilon(\boldsymbol{r})=\varepsilon(\boldsymbol{r} \pm \boldsymbol{a})$ Equation 2.21 
By repeating this translation, we see that $\varepsilon(\boldsymbol{r})=\varepsilon(\boldsymbol{r}+\mathbf{R})$ for any $\mathbf{R}$ that is an integral multiple of $a$; $\mathbf{R}=l \mathbf{a}$ where $l$ is an integer. The discrete periodicity in the y direction leads to a $y$-dependency for $\mathbf{H}$ that is simply the product of a plane wave with a $y$-periodic function. The plane wave is similar to that in free space but modulated by a periodic function because of the periodic lattice as shown in Equation 2.22 [4]

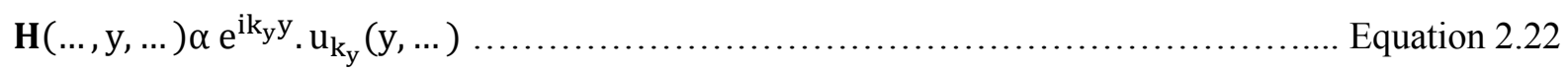

The modes can then be written as follows

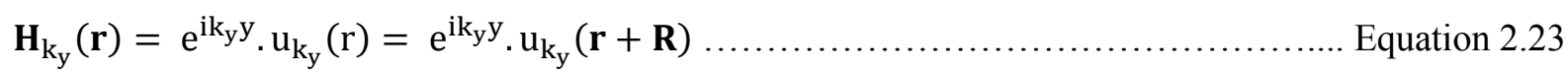

This result is commonly known as Bloch's theorem. In solid states physics, the form of Equation 2.22 is known as a Bloch state [3-6]. Bloch states with a wave vector $\mathrm{k}_{\mathrm{y}}$ and that of a wave vector $\mathrm{k}_{\mathrm{y}}+m b$ are identical. In fact, all the $\mathrm{k}_{\mathrm{y}}$ 's that differ by an integral multiple of $b=\frac{2 \pi}{a}$ are not different from a physical point of view. This allows us to consider only $\mathrm{k}_{\mathrm{y}}$ 's that exist in the range $-\frac{\pi}{a}<k_{y} \leq \pi / a$ which is the region of nonredundant values of $\mathrm{k}_{\mathrm{y}}$. This region is commonly known as the Brillouin zone, which is explained later in this section. In this region, the dielectric is invariant under translations through a multitude of lattice vectors and $\boldsymbol{R}=l a_{1}+m a_{2}+n a_{3}$ for some integers $l, m$, and $n$ [4].

The real space vectors, $a_{1}, a_{2}$, and $a_{3}$, give rise to three primitive reciprocal lattice vectors, $b_{1}, b_{2}$, and $\mathrm{b}_{3}$, defined in such a way that $\boldsymbol{a}_{\boldsymbol{i}} \cdot \boldsymbol{b}_{\boldsymbol{j}}=2 \pi \delta_{i j}$. These reciprocal vectors, which will also be explained later in this section, span a reciprocal lattice of their own which is inherited by wave vectors [3-6].

The modes of a three-dimensional periodic system are Bloch states that can be labeled by a Bloch wave vector $\mathbf{k}=k_{1} b_{1}+k_{2} b_{2}+k_{3} b_{3}$ where $\mathrm{k}$ lies in the Brillouin zone. Each value of the wave vector $\mathbf{k}$ inside the Brillouin zone identifies an eigenstate of $L$ with frequency $\omega(\mathbf{k})$ and an eigenvector $\mathbf{H}_{\mathbf{k}}$ of the form:

$\mathbf{H}_{\mathbf{k}}(\mathbf{r})=e^{i \mathbf{k} \cdot \mathbf{r}} \boldsymbol{u}_{k}(\mathbf{r})$ Equation 2.24

Where $\boldsymbol{u}_{\mathrm{k}}(\mathrm{r})$ is a periodic function on the lattice $\mathbf{u}_{\mathbf{k}}(\mathbf{r})=\boldsymbol{u}_{k}(\mathbf{r}+\mathbf{R})$ for all lattice vectors $\mathbf{R}$. The wave vector $\mathbf{k}$ is a conserved quantity in a periodic system and hence the addition of a reciprocal lattice vector does not change an eigenstate or its propagation direction. This is different from the free-space case, in which all wave vectors represent physically distinct states [3]. 
Equation 2.24 demonstrates how the electromagnetic modes of a photonic crystal with discrete periodicity can be written as Bloch states. All of the information about these modes is given by the wave vector $\mathbf{k}$ and the periodic function $\mathbf{u}_{\mathrm{k}}(\mathbf{r})$. To solve for $\mathbf{u}_{\mathrm{k}}(\mathbf{r})$, the Bloch state can be inserted into the master equation to get the following:

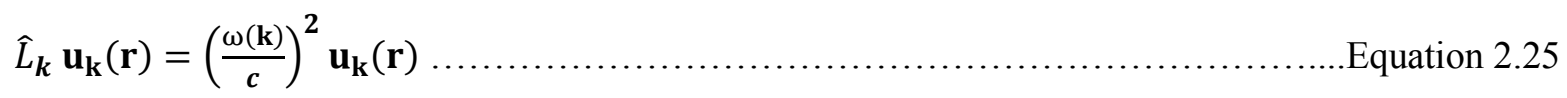

Where $\widehat{L}_{k}$ is the new Hermitian operator defined as $\hat{L}_{k}=(i \mathbf{k}+\nabla) \times \frac{1}{\varepsilon(\mathbf{r})}(i \mathbf{k}+\nabla) \times$

The function $\boldsymbol{u}$ and the mode profiles are determined by the eigenvalue problem which is restricted to a single unit cell of the photonic crystal lattice to create a discrete spectrum of eigenvalues. Hence, for each value of $\mathbf{k}$, an infinite set of modes with discretely spaced frequencies can be found, which we can label by a band index $\mathrm{n}$. And since $\mathbf{k}$ enters as a continuous parameter in $\hat{L}$, the frequency of each band varies continuously as $\mathbf{k}$ varies. Therefore, the modes of a photonic crystal are simply a set of continuous functions $\omega_{n}(\mathbf{k})$ indexed in the order of increasing frequency by the band number. The information contained in these functions is called the band structure of the photonic crystal. Studying the band structure of a crystal supplies us with the most of the information we need to predict its optical properties.

For a given photonic crystal with a periodic dielectric functione(r), we can use powerful software computational tools that solve Equation 2.25 as a standard eigenvalue equation in an iterative minimization technique for each value of $\mathbf{k}$. The electromagnetic variational theorem states that "the lowest frequency mode of a system is the field pattern that minimizes the electromagnetic energy functional," [39] and this is accomplished by concentrating electric field energy in the high dielectric regions. Therefore, the definition of a photonic crystal's dielectric and air bands is analogous to a semiconductor's valence and conduction bands. A band diagram of a two-dimensional infinitely long photonic crystal of triangular lattice of air holes in silicon is shown in Figure 2.2. In this figure, the lower frequency dielectric bands have their electric field energy mostly concentrated in higher dielectric regions, while higher frequency air bands have the electric field energy of their modes concentrated in lower dielectric regions.

The photonic bandgap is that frequency range in the middle in which there are no solutions for the master photonic crystal equation for any $\mathbf{k}$ value as shown in Figure 2.3. The origin of the photonic bandgap arises due to the fact that the lower frequency band's electric field energy becomes concentrated in the higher dielectric regions, and since the electromagnetic variational theorem requires that each allowable mode be orthogonal to the modes below it in frequency, the second band modes would ideally be concentrated in the same high dielectric regions as well [39]. However, the orthogonality requirement 


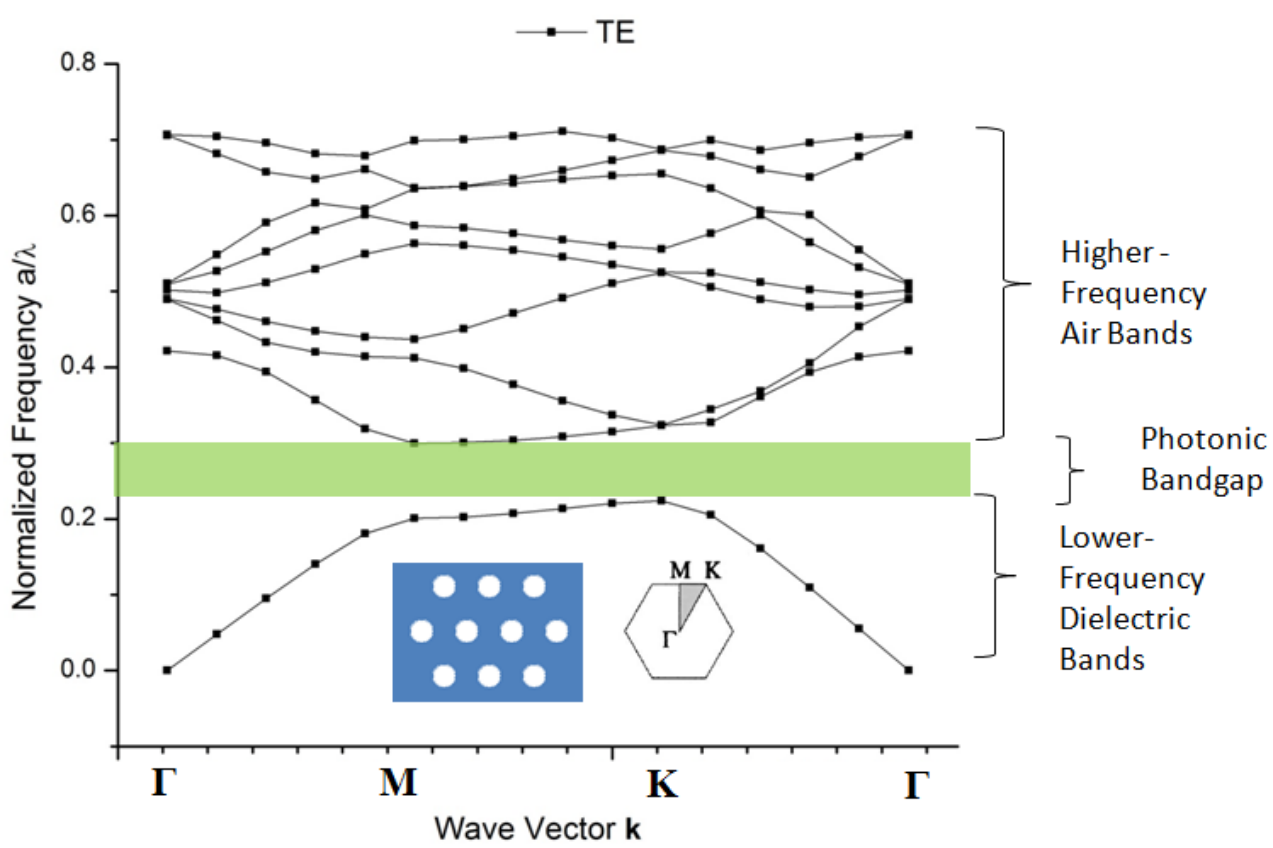

Figure 2.2. TE band diagram of a two-dimensional infinitely-thick photonic crystal of triangular lattice of air holes in silicon. Green band indicate the region of no solutions (photonic bandgap)

causes the electric field energy of this second band of modes to be concentrated in the lower dielectric regions instead. A significant increase in the frequency of the second band compared to the first would then be sufficient to create a region of no modes that is known as the 'photonic bandgap'.

\section{The Reciprocal Lattice}

If $f(\mathbf{r})$ is a periodic function on a lattice with $f(\boldsymbol{r})=f(\boldsymbol{r}+\boldsymbol{R})$ for all vectors $\mathbf{R}$ that connect one lattice point to the next, then the dielectric function $\varepsilon(\mathbf{r})$ in photonic crystals is considered an example of such a function [3]. To analyze any periodic function, it is common to take the Fourier transform and build a periodic function $f(\boldsymbol{r})$ out of plane waves with various wave vectors. Given a lattice with a set of lattice vectors $\mathbf{R}$, all of the reciprocal lattice vectors $G$ can be determined once the condition $G \cdot R$ is some integer multiple of $2 \pi$ for every $\mathbf{R}$. Every lattice vector $\mathbf{R}$ can be written in terms of primitive lattice vectors, which are the smallest set of vectors pointing from one lattice to another. For example, on a simple cubic lattice with a lattice constant $a$, the vectors $\mathbf{R}$ are of the form: $\mathbf{R}=l a_{1} \hat{x}+m a_{2} \hat{y}+n a_{3} \hat{z}$ where $(l, m, n)$ are integers and $\mathrm{a}_{1}, \mathrm{a}_{2}$, and $\mathrm{a}_{3}$ are the primitive lattice vectors [4].

The reciprocal lattice vectors $\{\mathbf{G}\}$ form a lattice of their own. In fact, the reciprocal lattice has a set of primitive vectors $b_{i}$ that will allow $\mathbf{G}$ to be written as $G=l b_{1}+m b_{2}+n b_{3}$. The requirement that $\mathbf{G} \cdot \mathbf{R}=2 \pi N$ will result with the following: [4] 


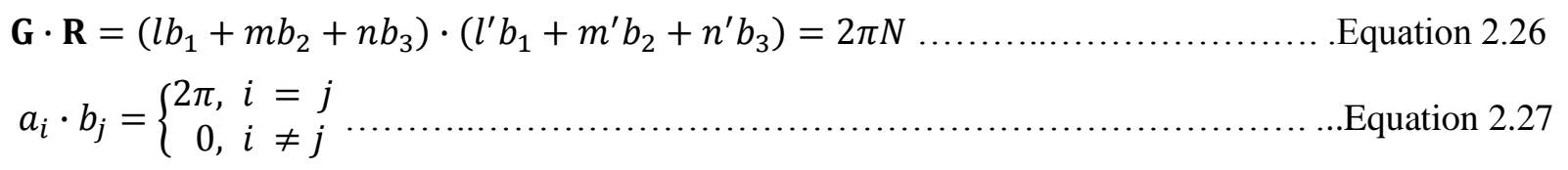

Equation 2.27 can be written as $a_{i} \cdot b_{j}=2 \pi \delta_{\mathrm{ij}}$. From there, given the set $\left\{\mathrm{a}_{1}, \mathrm{a}_{2}, \mathrm{a}_{3}\right\}$, the set $\left\{\mathrm{b}_{1}, \mathrm{~b}_{2}\right.$, $\left.b_{3}\right\}$ must be identified to satisfy the condition of Equation 2.27. One method of resolving this can be done by exploiting a feature of the cross product [4]:

$\mathbf{x} \cdot(\mathbf{x} \times \mathbf{y})=0$

Equation 2.28

Therefore, for any vectors $\mathbf{x}$ and $\mathbf{y}$ we can construct the primitive reciprocal lattice vectors with the following recipe:
$\mathrm{b}_{1}=\frac{2 \pi a_{2} \times a_{3}}{a_{1} \cdot\left(a_{2} \times a_{3}\right)}$
$\mathrm{b}_{2}=\frac{2 \pi a_{3} \times a_{1}}{a_{1} \cdot\left(a_{2} \times a_{3}\right)}$
$\mathrm{b}_{3}=\frac{2 \pi a_{1} \times a_{2}}{a_{1} \cdot\left(a_{2} \times a_{3}\right)}$

\section{The Brillouin Zone}

One interesting feature of Bloch states is that different values of $\mathbf{k}$ can lead to the same mode. For example, a mode with wave vector $\mathbf{k}$ and a mode with a wave vector $\mathbf{k}+\mathbf{G}$ are considered the same mode, if $\mathbf{G}$ is a reciprocal lattice vector. Therefore, when $\mathbf{k}$ is incremented by $\mathbf{G}$, the phase between cells is incremented by $\mathbf{G} * \mathbf{R}$ which is $2 \pi N$ and the result is the same physical mode. This means that there is a redundancy in the label $\mathbf{k}$ [4]. To eliminate this redundancy, a finite zone in reciprocal space must be found in which adding increments of $\mathbf{G}$ will not lead to the same mode. The zone that is closest to $\mathrm{k}=0$ is called the (first) Brillouin zone. And since the work presented here focuses on the square and triangular lattices, the reciprocal lattice vectors and Brillouin zones of each must be identified.

For a square lattices with a lattice constant $\boldsymbol{a}$, the lattice vectors are $a_{1}=a \hat{x}$ and $a_{2}=a \hat{y}$. To use the prescription of identifying reciprocal vectors as stated in the previous section, a third basis $a_{3}$ in the $\mathrm{z}$ direction must be used of any length, since the crystal is homogeneous in that direction. The results are

$$
\mathrm{b}_{1}=\frac{2 \pi}{a} \hat{\mathbf{x}} \quad \mathrm{b}_{2}=\frac{2 \pi}{a} \hat{\mathbf{y}}
$$


The reciprocal lattice is also a square lattice, but with spacing $\frac{2 \pi}{a}$ instead of $\boldsymbol{a}$ and hence the name reciprocal lattice suits this fact very well. The same analogy can be applied to the triangular lattice with lattice vectors $(\hat{\boldsymbol{x}}+\hat{\boldsymbol{y}} \sqrt{3}) a / 2$ and $(\hat{\mathbf{x}}-\hat{\mathbf{y}} \sqrt{3}) a / 2$ to obtain the reciprocal lattice vectors [4]

$$
\mathrm{b}_{1}=\frac{2 \pi}{a}\left(\hat{\mathbf{x}}+\frac{\hat{\mathrm{y}}}{\sqrt{3}}\right) \quad \mathrm{b}_{2}=\frac{2 \pi}{a}\left(\hat{\mathbf{x}}-\frac{\hat{\mathrm{y}}}{\sqrt{3}}\right)
$$

This is a triangular lattice rotated by $90^{\circ}$ with respect to the primitive one with spacing of $\frac{4 \pi}{a \sqrt{3}}$.

To determine the Brillouin zone, the origin point is selected and the area that is closest to this point than any other point in the lattice is shaded. Geometrically, this can be accomplished by drawing perpendicular bisectors of every lattice vector that starts at that origin point. Each bisector divides the lattice into two half-planes and the intersection of all the half-planes that contain origin is the Brillouin zone. The square and triangular primitive lattice vectors, reciprocal vectors, and the Brillouin zones are shown in Figure 2.3.

In some photonic crystal lattices, we need not consider every point of $\mathrm{k}$ inside the Brillouin zone. The smallest region within the Brillouin zone for which the $\omega_{n}(\mathbf{k})$ are not related by symmetry is called the irreducible Brillouin zone. A photonic crystal with the symmetry of a simple square lattice and a square Brillouin zone center at $\mathbf{k}=0$, the irreducible zone is a triangular wedge with $1 / 8$ the area of the full Brillouin zone; the rest of the Brillouin zone consists of redundant copies of the irreducible zone.

\subsubsection{Two-dimensional Photonic Crystals (Infinitely Long)}

A two-dimensional photonic crystal is periodic along two of its axes and homogenous along its third axis (Figure 2.4). Any mode that propagates parallel to the plane of the photonic crystal is invariant under reflections through that plane [4]. The mirror symmetry of the $2 \mathrm{D} \mathrm{PhC}$ lattices allows for the classification of the modes into two polarizations; transverse-electric (TE) modes having $\mathbf{H}$ normal to the plane and $\mathbf{E}$ in the plane and transverse-magnetic (TM) modes have just the opposite of that [4]. The band diagrams for TE and TM modes can be completely different. It is therefore possible to observe a wide photonic bandgap for one polarization and not for the other for a specific lattice. In this section, the band diagram of an infinitely-long photonic crystal will be discussed.

Only very few geometries can be analyzed using exact analytical tools. For most photonic crystals, numerical simulations are required to fully understand the behavior of light that interacts with them. The plane-wave expansion method, which makes use of the fact that normal modes in periodic structures can 


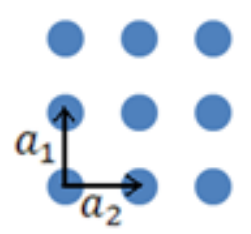

$a_{1}=a \hat{x}$

$a_{2}=a \hat{y}$

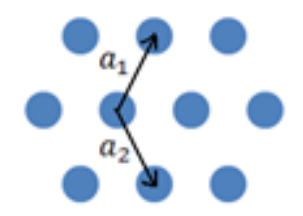

$$
\begin{aligned}
& a_{1}=(\hat{\mathbf{x}}+\hat{\mathbf{y}} \sqrt{3}) a / 2 \\
& a_{2}=(\hat{\mathbf{x}}-\hat{\mathbf{y}} \sqrt{3}) a / 2
\end{aligned}
$$

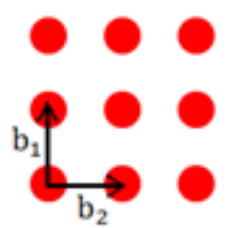

$\mathrm{b}_{1}=\frac{2 \pi}{a} \mathrm{Q}$

$\mathrm{b}_{2}=\frac{2 \pi}{a} \mathrm{y}$

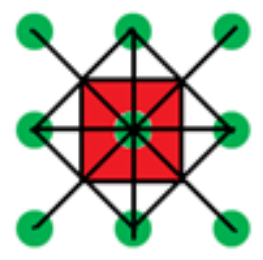

First Brillouin Zose

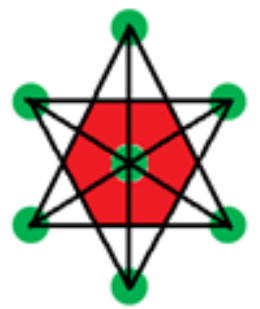

$$
\mathrm{b}_{1}=\frac{2 \pi}{a}\left(\hat{\mathrm{x}}+\frac{\hat{\mathrm{y}}}{\sqrt{3}}\right)
$$

$\mathrm{b}_{2}=\frac{2 \pi}{a}\left(\hat{\mathrm{x}}-\frac{\hat{\mathrm{y}}}{\sqrt{3}}\right)$
First Brillouin Zone

Figure 2.3. The primitive lattice vectors, the reciprocal lattice vectors, and the Brillouin zones for the square (top row) and triangular lattices (bottom row) [4]

be expressed as a superposition of a set of plane waves is the most convenient method to understand this light interaction with periodic $\mathrm{PhC}$ lattices [3-6].

In this method, the master equation is converted to a matrix eigenvalue problem that is solved using standard numerical techniques to obtain the dispersion relations inside a photonic crystal. The eigenfrequency solutions are plotted as functions of the in-plane wave vectors tracing the edges of the irreducible Brillouin zone. The resulting dispersion diagram becomes simply a geographical representation of the frequencies that correspond to waves propagating within a photonic crystal lattice with various wave vectors.

The diagrams for square and triangular lattices of rods of Si surrounded by toluene are shown in Figure 2.5. It is obvious how a complete overlapping bandgap cannot be extracted from either lattice. The minima and maxima of the first and second bands, respectively, determine the bandgap frequencies and almost always occur at the irreducible Brillouin zone edges, and often at a corner. Therefore, the wave vector is normally plotted along the edges of this irreducible Brillouin zone. 


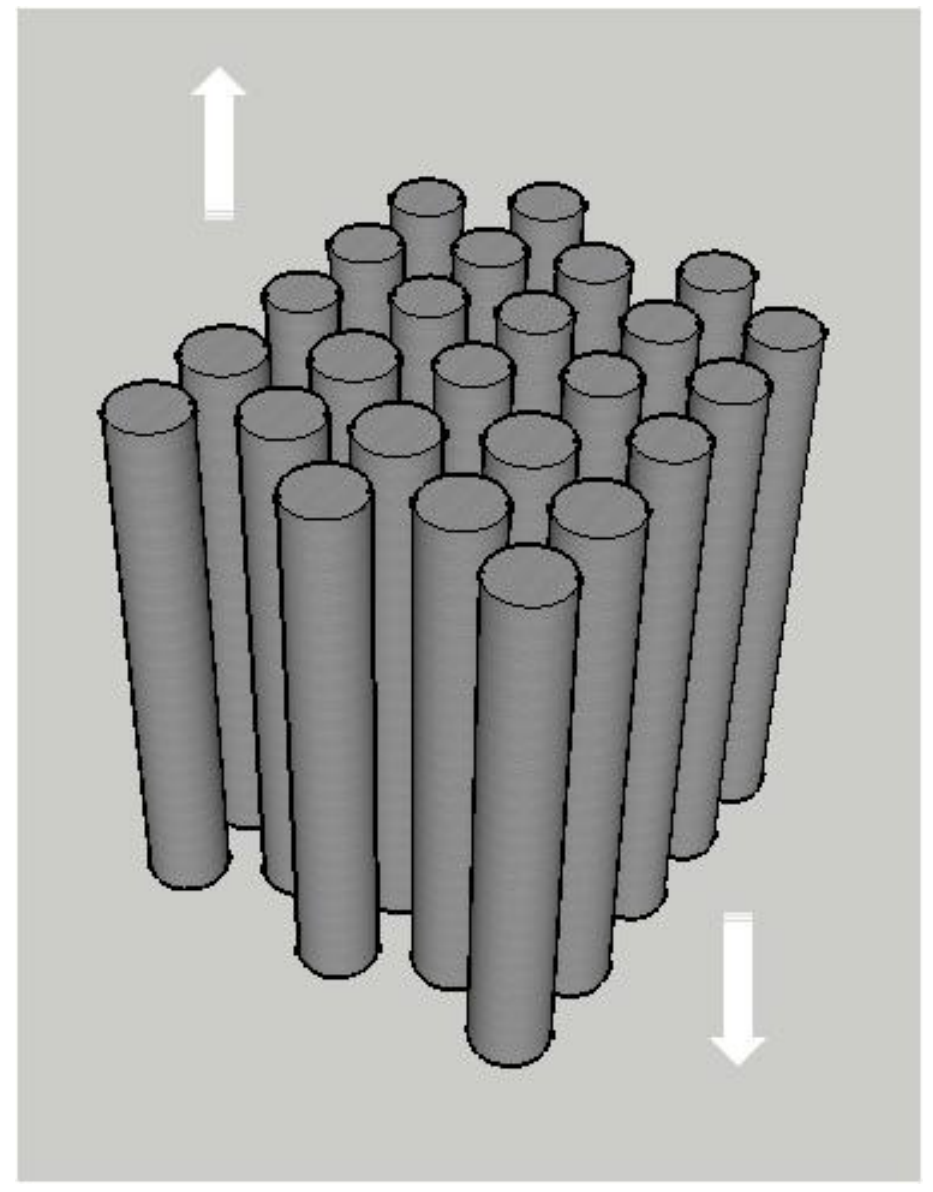

Figure 2.4. Infinitely-long $2 \mathrm{D} \mathrm{PhC}$ with a triangular lattice symmetry of dielectric rods 
Band Diagram for an Infinitely-Long Square Lattice Photonic Crystal of Si $(\varepsilon=12.5)$ pillars in Toluene $(\varepsilon=2.241)$

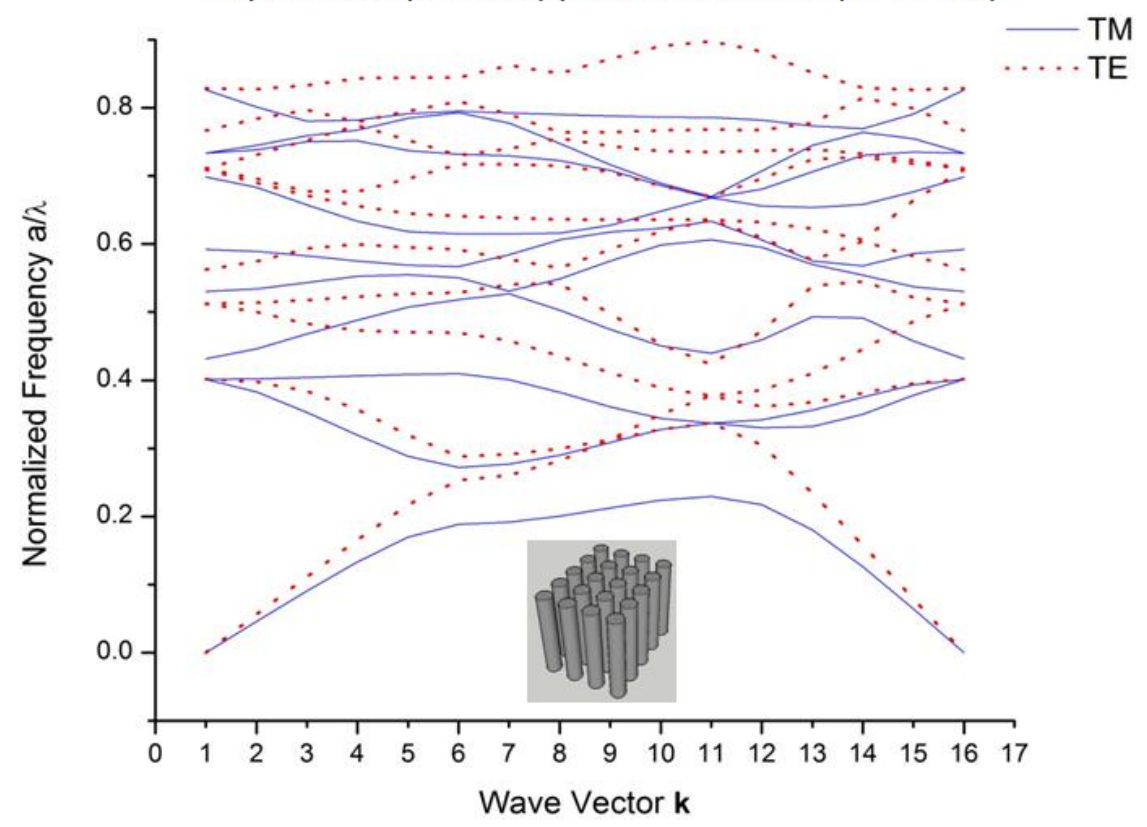

Band Diagram for an Infinitely-Long Triangular Lattice

Photonic Crystal of Si $(\varepsilon=12.5)$ pillars in Toluene $(\varepsilon=2.241)$

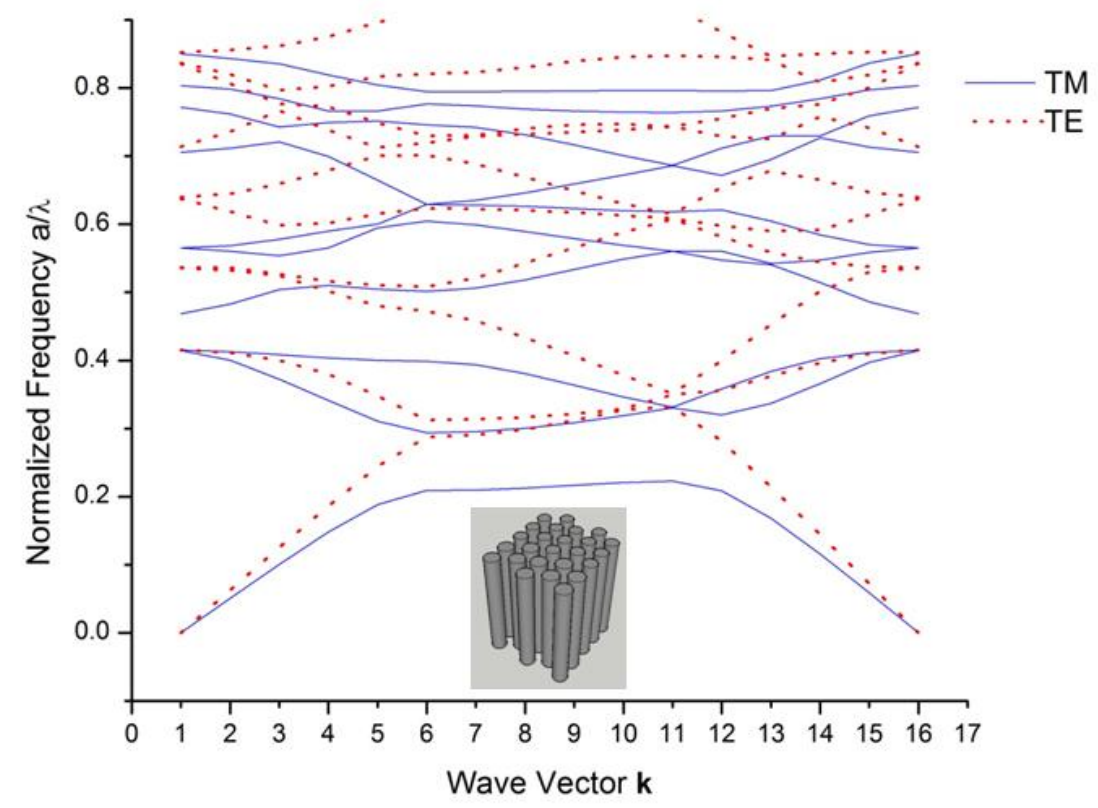

Figure 2.5. PWEM solved using MIT Photonic Bands (MPB) to obtain the dispersion (band) diagram for two different lattices. (top) Square lattice of silicon rods surrounded by toluene and (bottom) triangular lattice of silicon rods surrounded by toluene. Bandgap can 


\subsubsection{Two-dimensional Photonic Crystal Slabs}

Two-dimensional photonic crystal slabs (Figure 2.6) are similar to infinitely-long photonic crystals in their in-plane periodicity. However, two-dimensional calculations cannot be applied directly to finitethickness slab structures. In the previous section, the band diagram of a two-dimensional photonic crystal structure was shown in Figure 2.5 which may be applied to a three-dimensional structure only when these periodic structures extend infinitely in that third dimension [49]. These previous diagrams demonstrated two-dimensional bands that correspond to states with no vertical wave-vector component that is perpendicular to the $\mathrm{PhC}$ plane. When vertical wave vectors are included in the calculations, a continuum of states is produced throughout all frequencies above the first band and this ultimately cancels the bandgap of the two-dimensional structure [47-49]. Moving from an infinitely long structure to one with a finite thickness recreates the band gap in the guided slab modes. The system then becomes fundamentally three-dimensional and distinct from the two dimensional calculations with a new set of parameters that must be considered such as: slab thickness, effective refractive index contrast of the slab and its background, and mirror symmetry of the photonic crystal slab [49].

The band diagram of a PhC slab is calculated by first finding the states of the slab and then the light cone is overlaid as an opaque region on the band diagram [47]. The light cone consists of radiation 'leaky' modes that extend infinitely in the region above and below the slab. This region is normally referred to as the background. Guided modes, on the other hand, are simply the localized states within the slab and exist in the regions of the band diagram that are outside the light cone. The slab guided modes

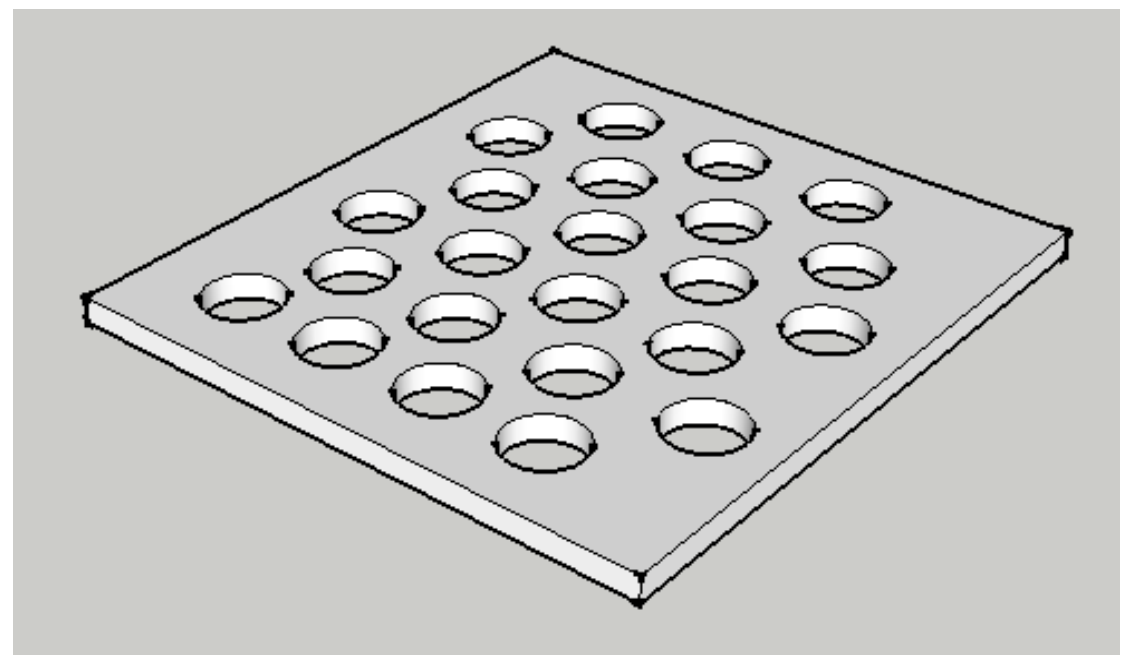

Figure 2.6. A two-dimensional photonic crystal slab 
are computed using preconditioned conjugate-gradient minimization of the Rayleigh quotient in a planewave basis [47]. This requires a periodic cell which is already achieved in the plane of the $\mathrm{PhC}$ and this periodicity is extended to the third dimension by generating a sequence of slabs separated by a background region (low- $\varepsilon$ medium). It is important that this background is of a sufficient thickness so that the periodic vertical slabs do not affect the mode solutions of each other.

The light cone is normally depicted as a uniform shaded area that does not reflect the varying density of states in this region. Figure 2.7 demonstrates the TE band diagram of a slab photonic crystal compared to that of an infinitely-long photonic crystal of a triangular lattice of toluene holes in silicon.

The light cone is the most important feature in Figure 2.8 that distinguishes slabs from infinitelylong photonic crystals. The guided modes that lie below the light cone in the bottom band diagram of Figure 2.7 do not couple with modes in the bulk background. These guided states extend infinitely in the $\mathrm{PhC}$ plane and decay exponentially into the background region [49]. The resulting confinement is somehow similar to the total internal reflection (TIR) in which the guided modes remain in the higher effective index of the silicon slab and become resonant when they reach the edge of the light cone but due to losses effects, they cannot remain resonant permanently within the slab and start to leak to the background. These structures are still referred to "photonic crystals" due to the existence of a photonic bandgap. This bandgap, however, is different than that for an infinitely-long lattice. It contains the range of frequencies in which no "guided" modes exist. Therefore, it is not a real bandgap as there are radiation losses which have a major impact on the overall functionality of the photonic crystal lattice. However, they have also been uniquely utilized by several research groups to successfully develop efficient light emitting diodes (LEDs) and lasers when they managed to control the background radiation using slab photonic crystals with defined bandgaps [3].

The guided modes within the PhC slab are divided into two classes that are not purely TE or TM polarized. The reason is due to the lack of a discrete translational symmetry in the vertical direction. However, when considering the horizontal symmetry plane that bisects the slab, guided modes then can be classified as even or odd with respect to reflections through this plane. These even and odd states are very similar to TE and TM states for the infinitely-long PhCs [48].

As mentioned earlier, the slab thickness plays an important role in determining whether or not a photonic bandgap exists for a specific PhC slab. Thick slabs will cause the generation of higher-order modes that lie slightly above the first band and hence either canceling or severely narrowing the photonic bandgap to a point of no use. On the other hand, thin slabs will be considered a very weak perturbation of the background dielectric constant and therefore guided modes within the slab will hardly exist [49]. Johnson et al [49] proposed a method of calculating the optimal slab thickness. It is suggested to be on the 

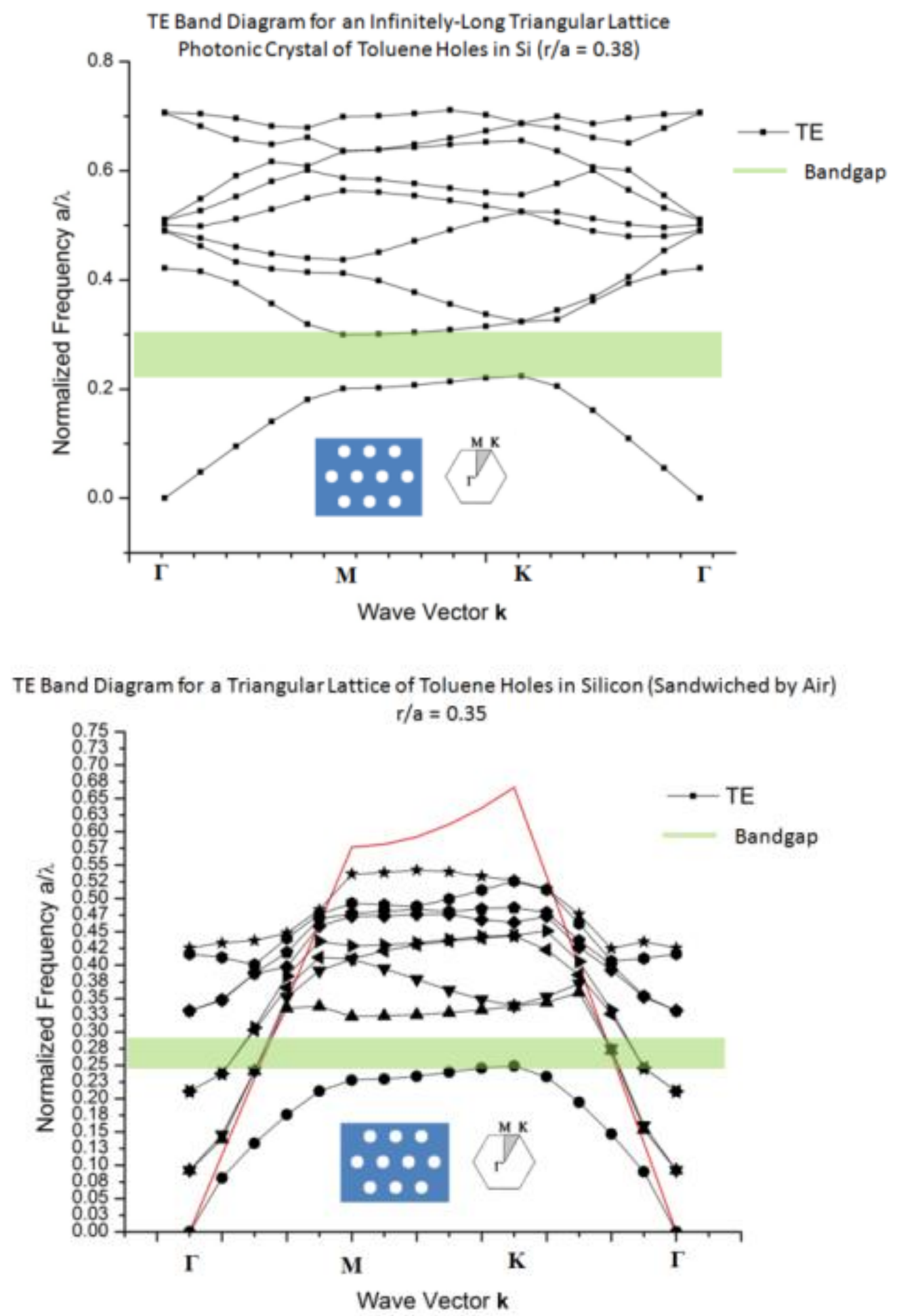

Figure 2.7. TE band diagram of a triangular lattice of an infinitely-long photonic crystal of toluene holes in Si (top)and a triangular lattice of a slab photonic crystal of toluene holes in Si (bottom). Photonic bandgaps are shaded in green. 
order of half the two-dimensional gap-bottom wavelength. The group justified this by explaining that the gap-bottom frequency was used instead of the midgap because the state at the bottom of the gap is the basis for both the state at the lower edge of the slab gap and the excited states at upper edge. When the slab thickness is at least an order of the wavelength, then the small energy barrier will prevent the generation of higher-order states via a nodal plane. On the other hand, slabs of less than half the wavelength in thickness will not be sufficient for the existence of any guided modes [49].

It is also very important to consider the effective refractive index of the background medium above and below the slab because in most applications, specifically in our proposed transducer as will be discussed in the following chapter, this medium will not be air at all times. Therefore, if the slab is sandwiched between some background with an effective refractive index that is lower than that of the slab, index guiding will still allow the generation of guided bands. However, it must be noted that the localization of the guided modes within the slab and the creation of bandgaps means that the substrate must be sufficiently thick (several wavelengths).

The increased effective refractive index surrounding the slab pulls down the frequencies of the guided modes and focuses them under the narrow light cone. However, the guided modes become less localized within the slab and it was measured that $89 \%$ of the energy of the lowest band remains within the height of the slab with a dielectric background of 2, versus $96 \%$ for that of an air background [49].

\subsubsection{Defects in Two-dimensional Photonic Crystals}

It has been discussed previously that a photonic bandgap of a two dimensional photonic crystal represents the range of wavelengths that are prohibited from propagating in the periodicity plane of the photonic crystal. This is mainly due to the fact that the dielectric periodicity of the photonic crystal causes the propagating light to undergo coherent scattering that prevents it from transmitting through the lattice. However, by breaking the lattice symmetry at specific locations, single localized modes or a set of closely spaced modes can be generated within the bandgap. This can be accomplished by either removing a single rod from a lattice of $\mathrm{PhC}$ rods within a specific background or replacing it with another one of different size, shape, or dielectric constant. This perturbation ruins the discrete translational symmetry of the lattice.

The main interest in these defects is the frequencies that they support as well as the associated quality factor (Q-factor) of each mode. As its name indicates, the Q-factor is simply a measure of the quality of this cavity or how efficient it is in storing energy. It is equal to the number of oscillations the cavity can support before the trapped energy decays by $e^{-2 \pi}(\sim 0.2 \%)$ [47]. The total quality factor for the cavity, $Q_{t o t}$, is represented as follows: 
$\frac{1}{Q_{t o t}}=\frac{1}{Q_{1}}+\frac{1}{Q_{2}}$

Equation 2.29

Where $Q_{1}$ is the $\mathrm{Q}$ factor of radiating modes outside of the slab and $Q_{2}$ is the quality factor of the guided modes in the plane of the photonic crystal. The in-plane leakage occurs due to tunneling effects. This leakage can be minimized by increasing the number of un-altered photonic crystal periods surrounding the cavity. The guided-modes' quality factor, $Q_{2}$, then becomes very large and its reciprocal in Equation 2.2, $1 / Q_{2}$, approaches zero making the following approximation valid [47]:

$Q_{t o t} \approx Q_{1}$ Equation 2.30

Another way of measuring a specific cavity's Q-factor is done by examining the sharpness of the cavity's resonance peak. The width of this peak is inversely proportional to the cavity $\mathrm{Q}$ factor,

$Q=\lambda_{0} / \Delta \lambda$ .Equation 2.31

Where $\lambda_{0}$ is the center resonant frequency and $\Delta \lambda$ is the spectral full-width half-maximum (FWHM) of the resonance. A high Q-factor photonic crystal nanocavity is the one that can maintain its trapped photons over more radiation cycles than a lower Q-factor nanocavity but can support much fewer resonant frequencies than the lower Q-factor cavities. These resonant cavities, in general, can be classified as acceptor or donor defects depending on their size and their index of refraction. Acceptor defects are formed by removal of high index material from a specific lattice location as shown in Figure 2.8(a) which represents an enlarged air hole within a two-dimensional triangular lattice of air-holes in silicon. The defect frequencies normally occur near the bottom edge of the photonic bandgap. On the other hand, donor defects are formed by the addition of high index material at a lattice location as shown in Figure 2.8(b). This defect is created by filling out a range of air holes with the same refractive index material as that of the slab, silicon here. Donor defect resonant frequencies normally occur near the top edge of the photonic band gap. 

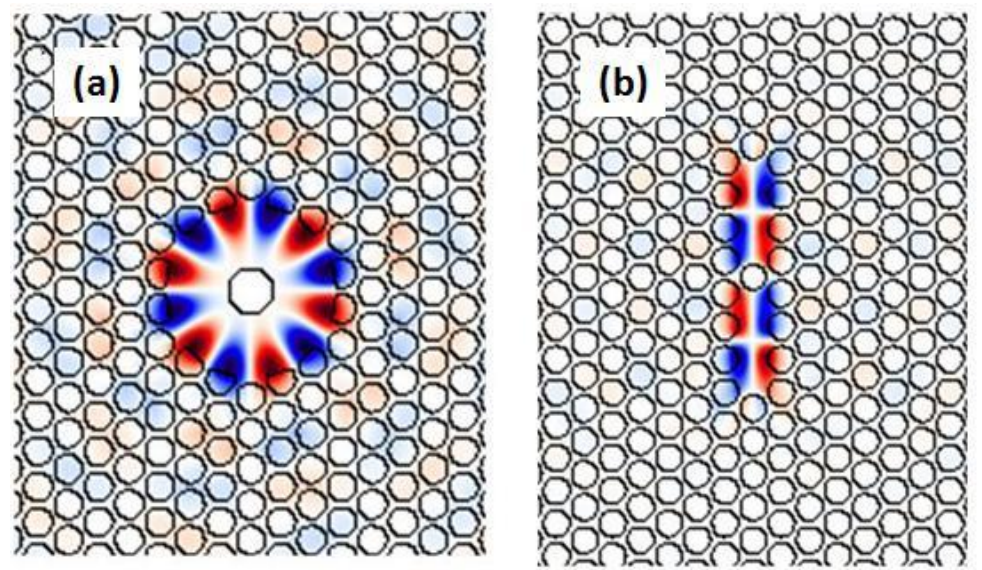

Figure 2.8. (a) a resonant cavity in which the defect is generated by filling in the holes with high dielectric material surrounding a central lower dielectric circular opening. (b) a resonant cavity in which 6 holes on top and bottom of a central hole are filled with high dielectric region. Extending this filling technique to the edge of the photonic crystal can generate a W1 waveguide.

\subsection{PHOTONIC CRYSTAL AS CORE STRUCTURES OF A BIOSENSOR}

Throughout this second chapter, the theory behind the unique characteristics of photonic crystals has been discussed. Utilizing these properties to achieve fluorescence enhancement will be the focus of the modeling, fabrication, and optical characterization results that will be discussed in the next few chapters. The modeling of various designs of semiconductor and polymer photonic crystal lattices is done first using frequency and time-domain simulation tools, MPB and MEEP respectively. These tools will provide us with critical information regarding the parameters of the photonic crystal lattice of each material, the bandgap ranges that they support, as well as the resonant cavity modes and their quality factors. Once this information is obtained, the results are translated into computer-aided-design (CAD) files that can be used to produce photonic crystal structures at the end of several fabrication processes, as will be explained in Chapter 4. The characterization of these structures using either PbS IR-emitting quantum dots (QDs) or fluorescein then follows to prove the concept of how these photonic crystals can become the core structures of an ultra-sensitive biosensor capable of enhancing fluorescence emitted from labeled biomolecules. 


\section{CHAPTER 3: THE MODELING OF PHOTONIC CRYSTAL LATTICES}

\subsection{SILICON BASED PHOTONIC CRYSTALS}

In the second chapter, the origin of the photonic band diagram was discussed. For a given photonic crystal with a periodic dielectric function $\varepsilon(\mathbf{r})$, powerful software computational tools are used to solve Equation 2.25 as a standard eigenvalue equation in an iterative minimization technique for each value of k. MIT Photonic Bands (MPB) is a freely available, frequency domain software tool used to carry out such simulation [50]. The $y$-axis of the diagram represents the normalized frequency, $a / \lambda$ where $a$ is the lattice constant, and the $\mathrm{x}$-axis is wave vector, $\mathbf{k}$.

When a bandgap is resolved for a specific lattice, a two-dimensional triangular lattice of air holes in Silicon for instance, the lattice constant can be calculated as well as the radius of the circular air holes. Infinitely-thick photonic crystal lattices are normally first simulated to have a rough approximation of where the bandgap will be. For most applications, an infinitely-thick photonic crystal lattice is not useful, yet it is a very critical starting point in simulation (Figure 3.1). The simulation results of these infinitelythick photonic crystals will be considered later on when simulating realistic two-dimensional photonic crystal slabs that can be fabricated and optically characterized.
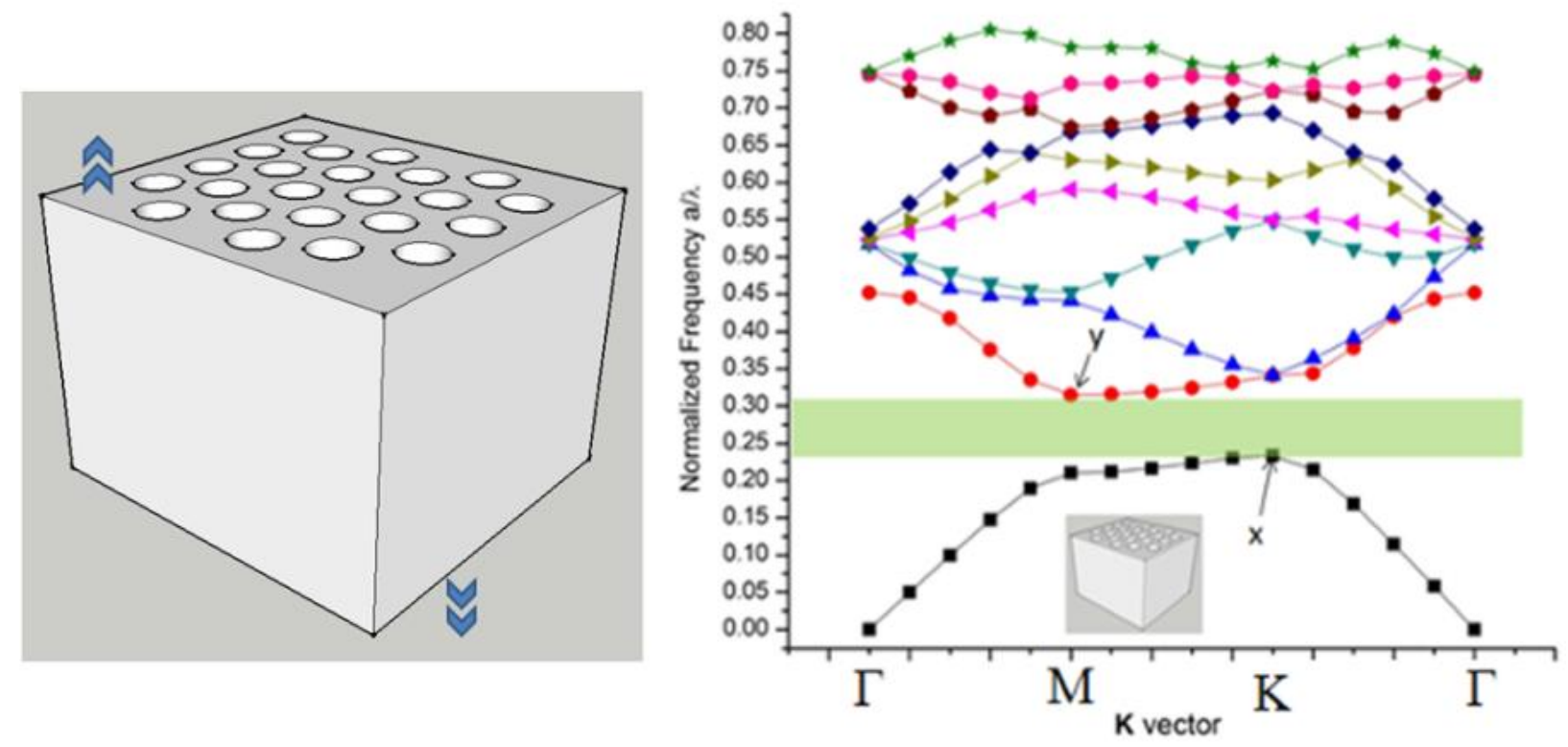

Figure 3.1. (Left) A representation of an infinitely-thick photonic crystal lattice of air holes in silicon. (Right) TE band diagram of an infinitely-thick photonic crystal of toluene holes in Silicon. Point $\mathrm{x}$ is the top of the lowest band while point $y$ represents the bottom of the second band. The difference is the photonic bandgap. 
The bandgap for a given lattice is typically characterized by its width, which can be calculated using the following formula [4] (using Figure 3.1 labels):

$$
\begin{aligned}
& \text { Bandgap Width (\%) } \\
& \begin{array}{r}
\text { Lowest frequency of higher order band - highest frequency of lower order band } \\
\text { Mid - gap frequency } \\
=\frac{x-y}{(x+y) / 2}
\end{array}
\end{aligned}
$$

\subsubsection{Infinitely-Thick Photonic Crystal Lattices of Silicon and Toluene}

For the silicon-based photonic crystal lattices, it is very critical to realize the optimal photonic crystal lattice and the critical parameters that will result with the widest possible complete photonic bandgap. This means that both polarizations should have an overlapping bandgap for a specific range of frequencies, and, the wider the bandgap, the better chance of enhancing more emitted frequencies from labeled biomolecules. However, it is important to note also that the widest possible bandgap for a specific lattice might not be practical since it may require a spacing between its high or low periodic dielectric regions beyond what can be fabricated and this imposes a serious limitation considering the difficulty of achieving sub-100nm $\mathrm{PhC}$ lattices with the available nano-fabrication tools. The most two common symmetric lattices that are investigated in this work are the square and the triangular lattices. Moreover, it is important to consider the possible refractive index of the low- $\varepsilon$ regions within these lattices as they will mostly be occupied by the solvent containing the target biomolecules. Later in Chapter 5, the characterization results will be demonstrated using $\mathrm{PbS}$ quantum dots (QDs) suspended in a toluene solution. Therefore, the low- $\varepsilon$ regions of the triangular and square lattices are assumed to be that of toluene throughout this section.

Modeling results of infinitely-thick lattices of either toluene holes in silicon or silicon pillars surrounded by toluene will be demonstrated for a specific range of radii. The four studied lattices are:

- Infinitely-thick square photonic crystal lattice of toluene holes in silicon

- Infinitely-thick square photonic crystal lattice of silicon pillars surrounded by toluene

- Infinitely-thick triangular photonic crystal lattice of toluene holes in silicon

- Infinitely-thick triangular photonic crystal lattice of silicon pillars surrounded by toluene 
To determine parameters necessary for design and fabrication, a photonic bandgap map will be first plotted. This map demonstrates the width of the TE and TM bandgaps for a range of lattice radii from $0.2 a$ to $0.4 a$. Once a wide bandgap is found for a specific lattice, the band diagram for the TE and TM bands is plotted separately and that specific lattice is indicted with a vertical line on the bandgap map plots. At the end of this section, tables containing numerical values of the bandgap widths and their locations will be demonstrated. Since the characterization of the silicon-based photonic crystal lattices is carried out using IR-emitting Quantum Dots (QDs) with an emission peak at around $1100 \mathrm{~nm}$, the actual lattice parameters that must be fabricated in order to confine this emission wavelength will also be tabulated at the end of each section.

For a square photonic-crystal lattice of toluene holes in an infinitely long theoretical silicon sample, the radius of the holes is varied from $0.2 a$ to $0.4 a$ in increments of 0.01 . The reason for these two boundaries is due to practicality of the fabricated structures once a bandgap is resolved. For example, to enhance an 1100-nm emitted light from the QDs, $0.2 a$ and $0.4 a$ demonstrate the range of radii of the square and triangular lattices that can be fabricated with the current available lithography tools. Beyond these two values, a complete bandgap may be observed, but fabricating a lattice with 'veins' or 'spots' [4] that are only very few tens of nanometers is a very challenging task and might be very unpractical once fabricated. The MPB modeling results of this structure revealed a very narrow bandgap for the TM polarization only between the $8^{\text {th }}$ and $9^{\text {th }}$ bands for a lattice with $r=0.35 a$. The band diagram of this only bandgap in this range is shown in Figure 3.2 (gap indicated with a red arrow).

For a square lattice of silicon pillars surrounded by toluene, bandgaps for both polarizations were observed for all lattice radii between $0.2 a$ and $0.4 a$. Most of the observed TE bandgaps, however, are between the higher bands that did not result with an overlapping complete photonic bandgap. The bandgap maps for both polarizations are shown in Figures 3.3 and 3.4. The largest TM bandgap of $18.57 \%$ was observed between the first and second bands for a lattice with $r=0.24 a$ (between normalized frequencies 0.243862 and 0.293771 ) while the largest TE bandgap of $5.076 \%$ was observed for the same lattice, $r=0.24 a$, but between the $9^{\text {th }}$ and $10^{\text {th }}$ bands. The band diagrams of these two polarizations are demonstrated in Figures 3.5 and 3.6.

For a triangular lattice of toluene holes in silicon, only very narrow bandgaps for the TM polarization were observed for the range of radii between 0.38 and 0.40 . The TE polarization, on the other hand, showed bandgaps for all radii values between $0.2 a$ and $0.4 a$. The bandgap map for each polarization is demonstrated in Figures 3.7 and 3.8 while the band diagram of the highest bandgap lattice for the TM and TE polarizations is demonstrated in Figures 3.9 and 3.10, respectively. However, no overlapping complete photonic bandgap for this lattice was observed. 
Finally, for a triangular lattice of silicon pillars surrounded by toluene, the TM polarization demonstrated bandgaps for the full studied range of radii while the TE bandgap demonstrated higherorder bandgaps for the range between $0.2 a$ and $0.25 a$ and $0.28 a$ and $0.30 a$ only. The bandgap map for each polarization is demonstrated in Figures 3.11 and 3.12 while the band diagram with widest bandgap for the TM and TE polarizations is demonstrated in Figures 3.13 and 3.14, respectively. However, once again, no overlapping complete photonic bandgap for this lattice was observed.

TM Band Diagram of a Square Lattice of Toluene Holes in Si $(r / a=0.35)$

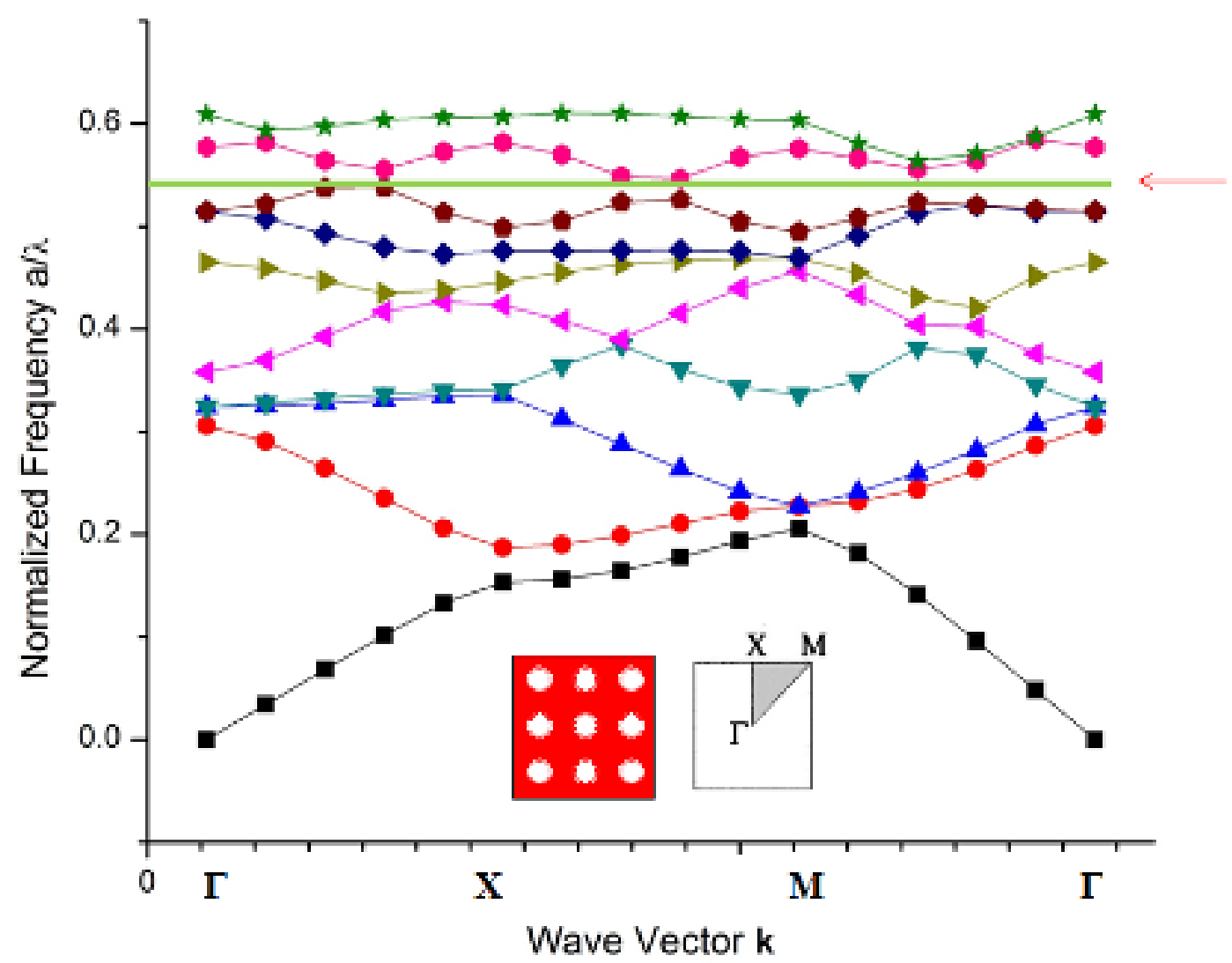

Figure 3.2. TM band diagram of a square lattice of toluene holes in Si. A very narrow bandgap is indicated with the red arrow between the $8^{\text {th }}$ and $9^{\text {th }}$ bands 
TM Bandgap Map for a Square Lattice of Silicon Pillars in Toluene

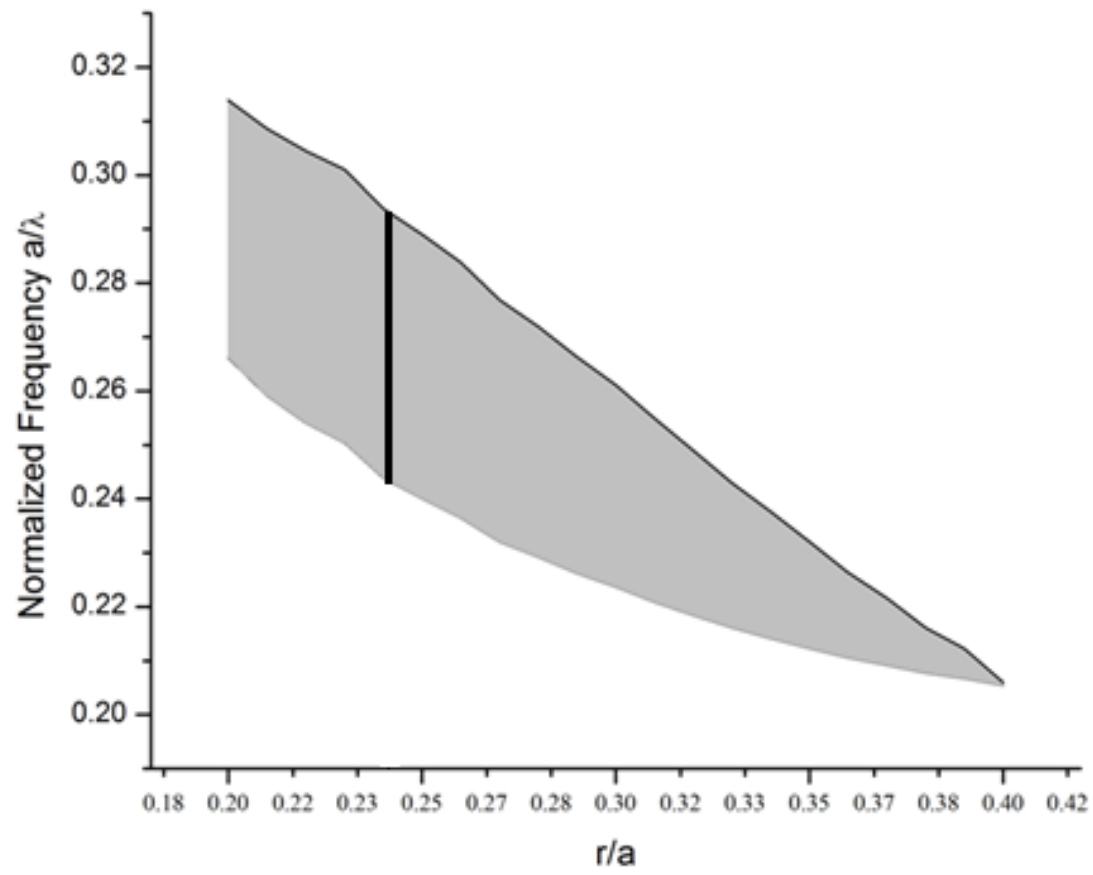

Figure 3.3. TM bandgap map for a square lattice of Si pillars in toluene. The widest observed bandgap was at r/a $=0.24$

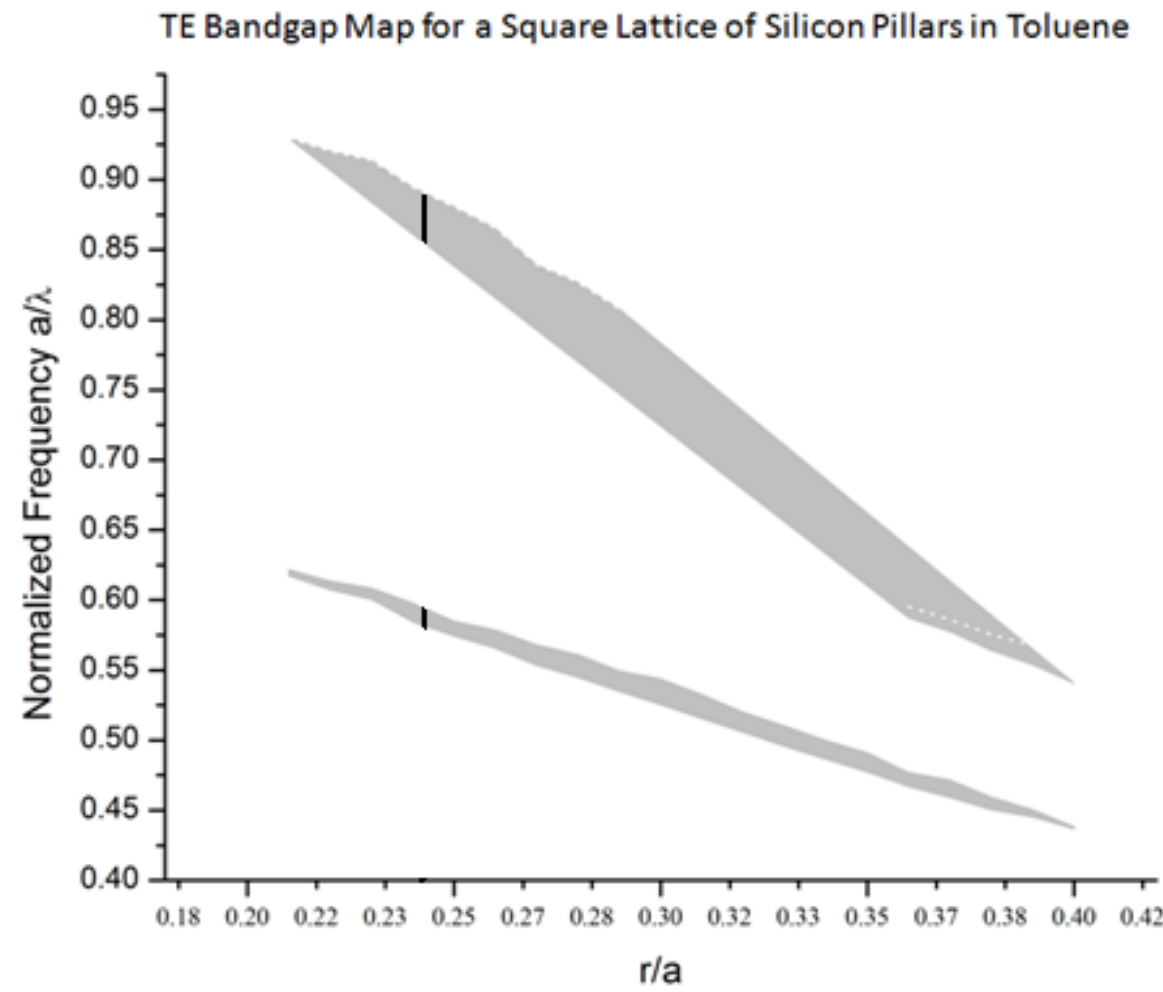

Figure 3.4. TE bandgap map for a square lattice of Si pillars in toluene. The two bandgaps at $\mathrm{r} / \mathrm{a}=0.24$ are indicated by a vertical line. 


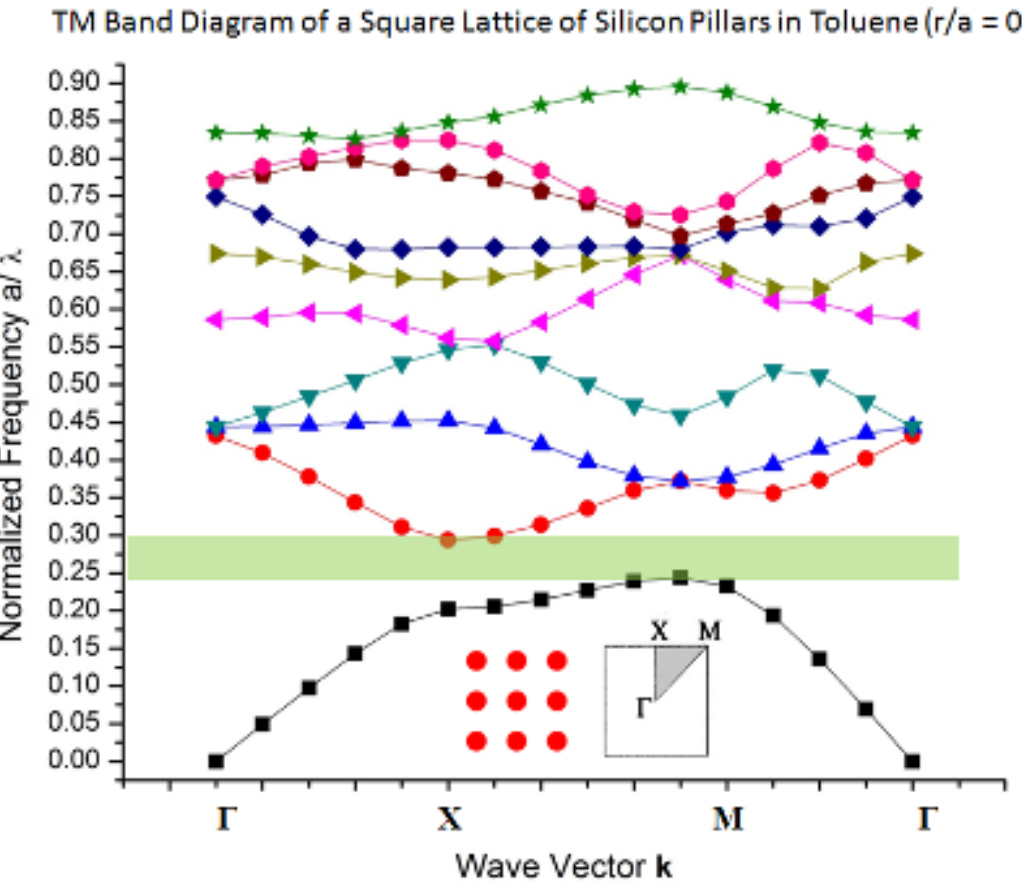

Figure 3.5. TM band diagram for $\mathrm{r} / \mathrm{a}=0.24$ with an $18.57 \%$ bandgap width between the first two bands

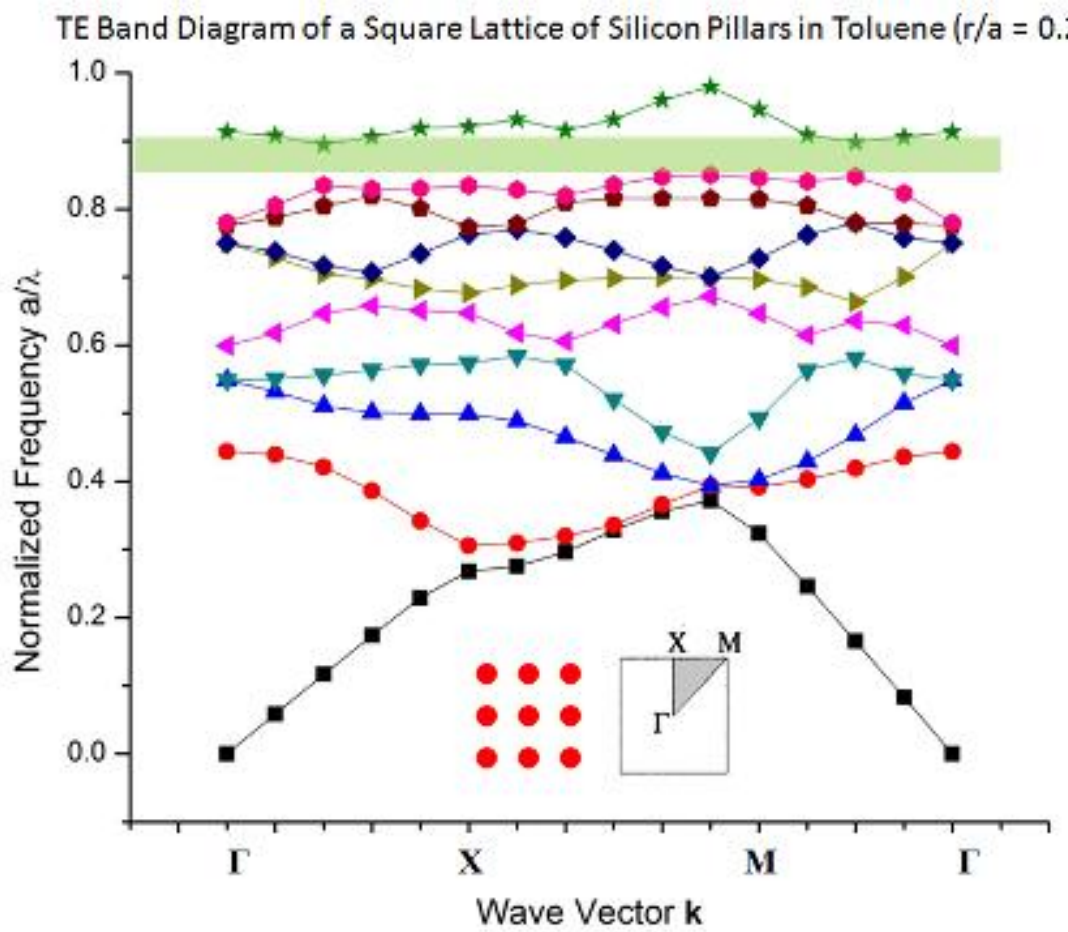

Figure 3.6. TE band diagram for $\mathrm{r} / \mathrm{a}=0.24$ with an $5.076 \%$ bandgap width between the $9^{\text {th }}$ and $10^{\text {th }}$ bands 


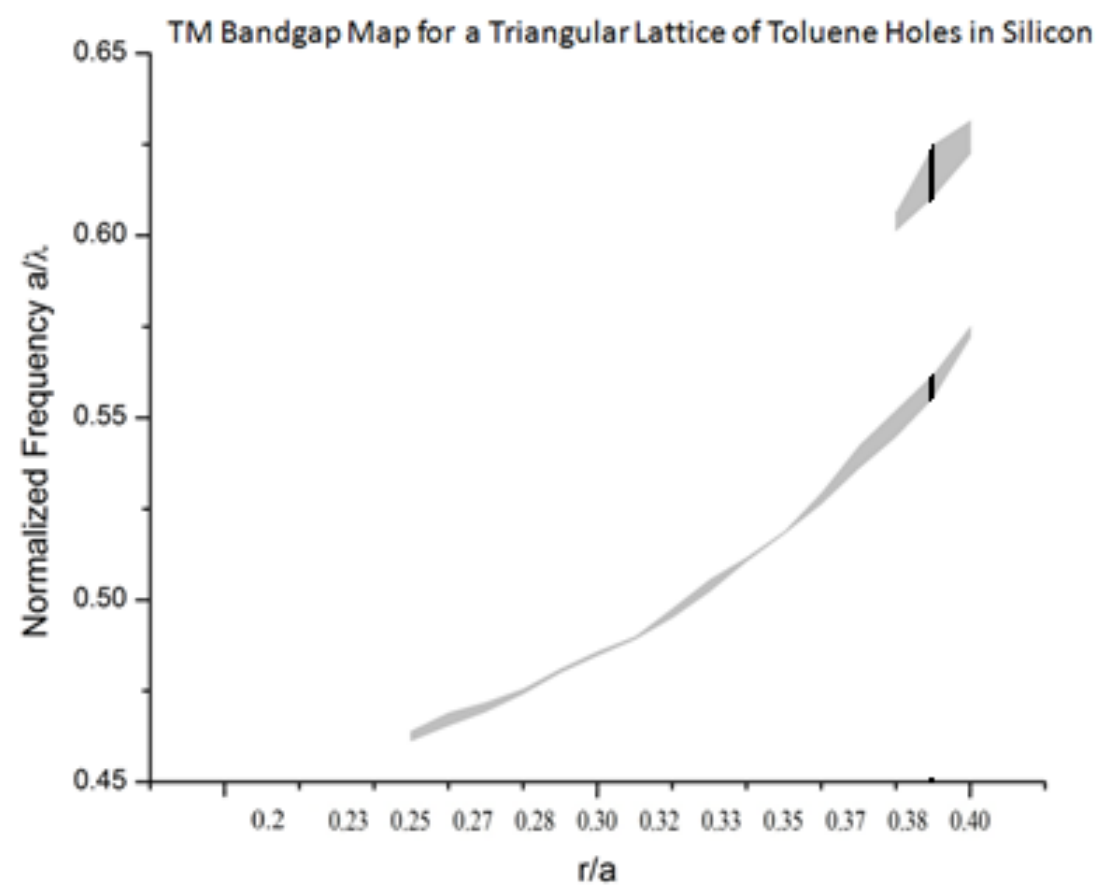

Figure 3.7. TM bandgap map of a triangular lattice of toluene holes in silicon. The widest observed bandgap was at $\mathrm{r} / \mathrm{a}=0.39$

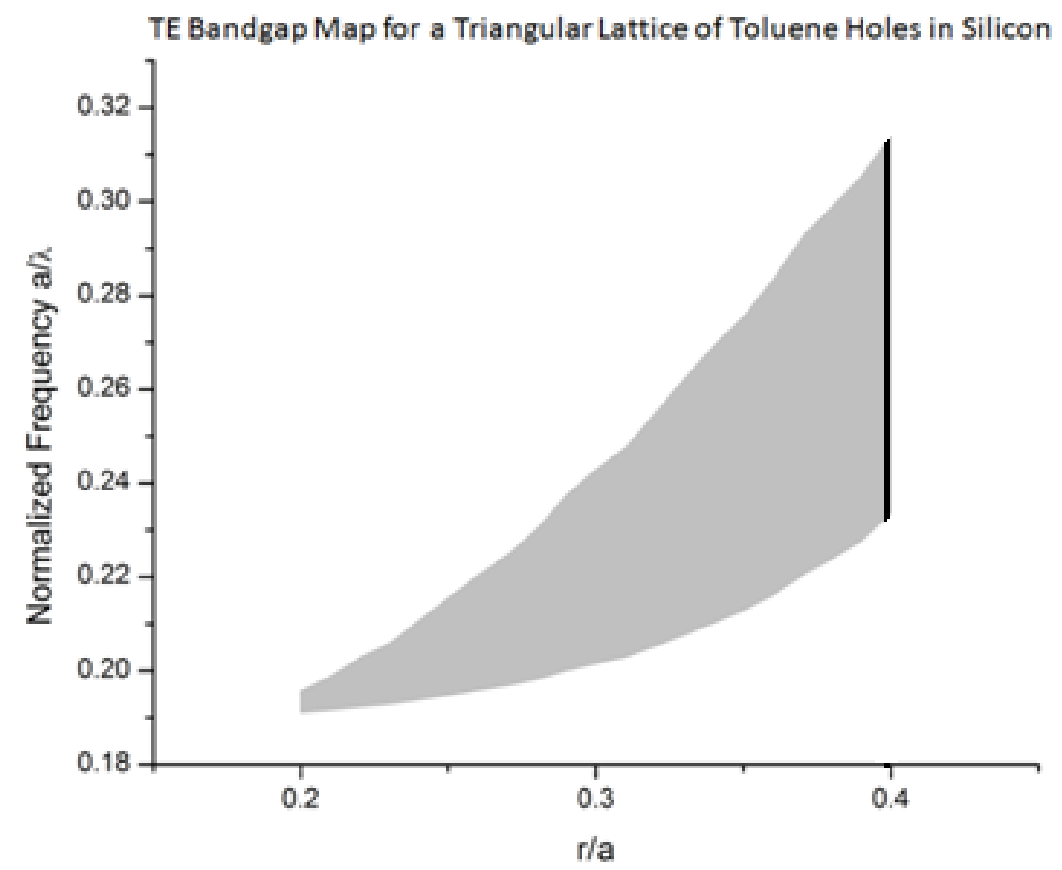

Figure 3.8. TM bandgap map of a triangular lattice of toluene holes in silicon.

The widest observed bandgap was at $\mathrm{r} / \mathrm{a}=0.4$ 


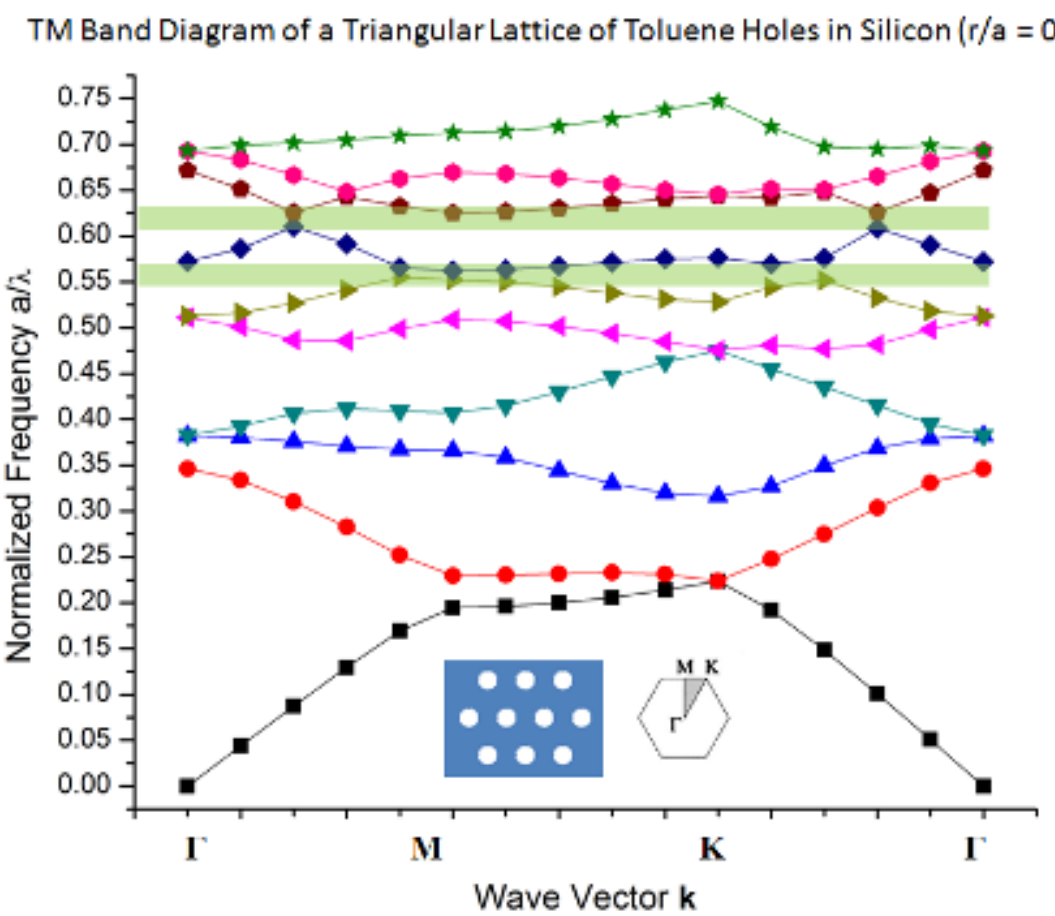

Figure 3.9. TM band diagram demonstrating two bandgaps between the higher-order bands ( 6 and 7 , and 8 and 9) for a triangular photonic crystal lattice of toluene holes in silicon with $\mathrm{r} / \mathrm{a}=0.39$

TE Band Diagram of a Triangular Lattice of Toluene Holes in Silicon ( $r / a=0.4)$

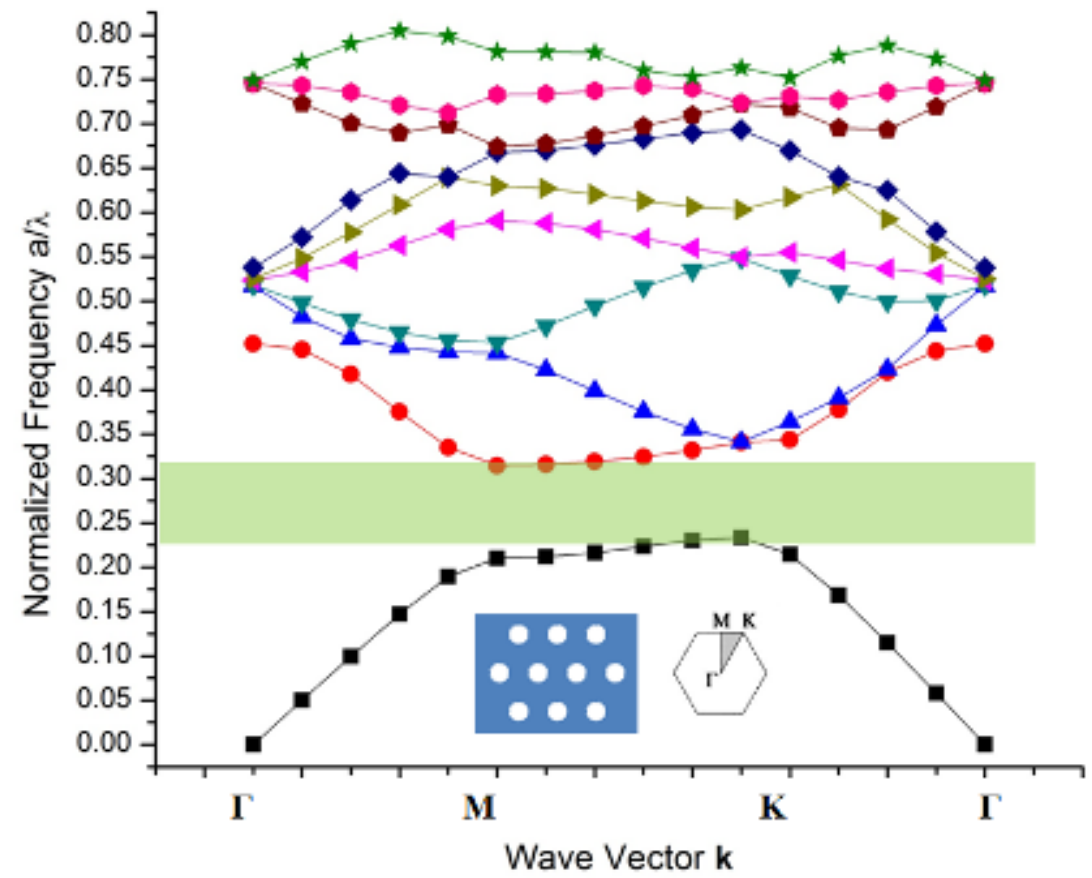

Figure 3.10. TE band diagram demonstrating a bandgap of size $29.59 \%$ between the first two bands for a triangular lattice of toluene holes in silicon with $\mathrm{r} / \mathrm{a}=0.4$ 


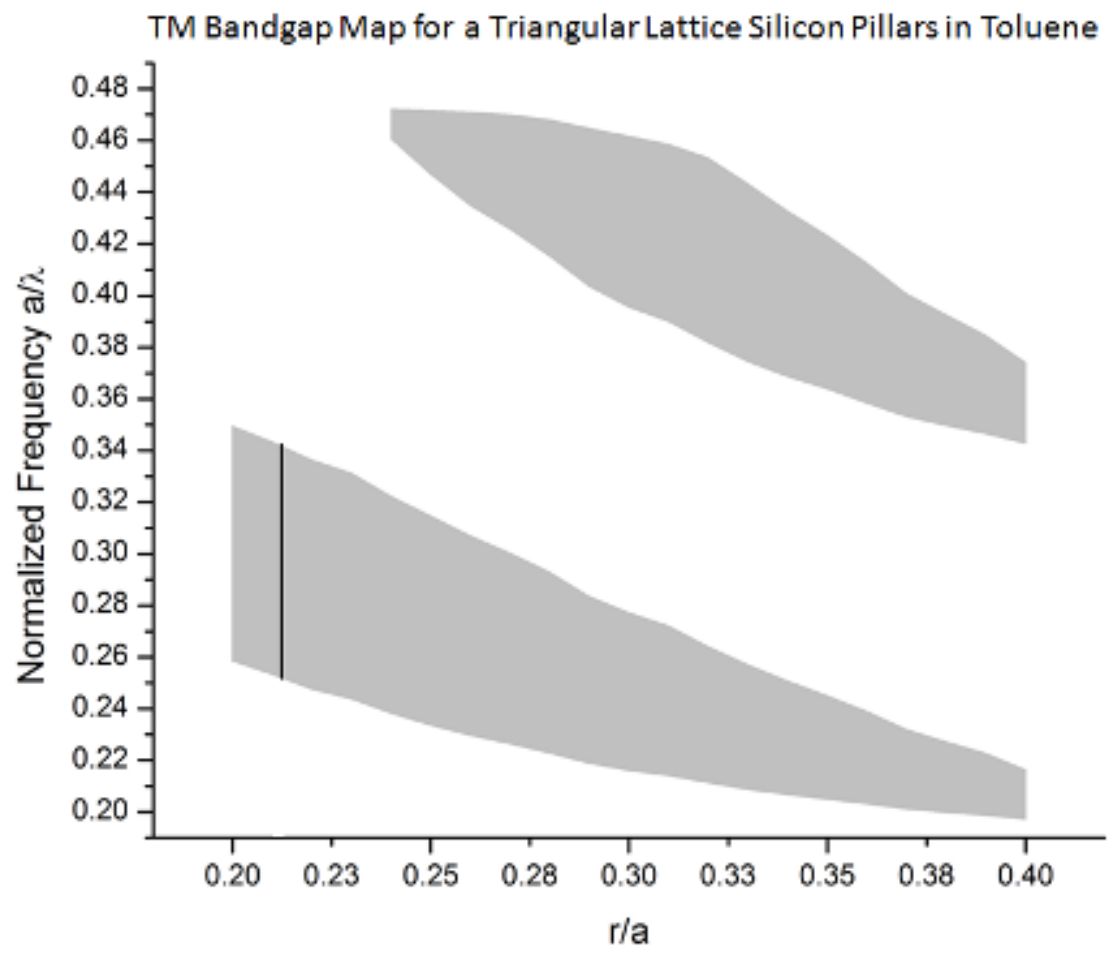

Figure 3.11. TM bandgap map for a triangular lattice of silicon pillars surrounded by toluene. The widest observed bandgap for this lattice was found at $\mathrm{r} / \mathrm{a}=0.22$

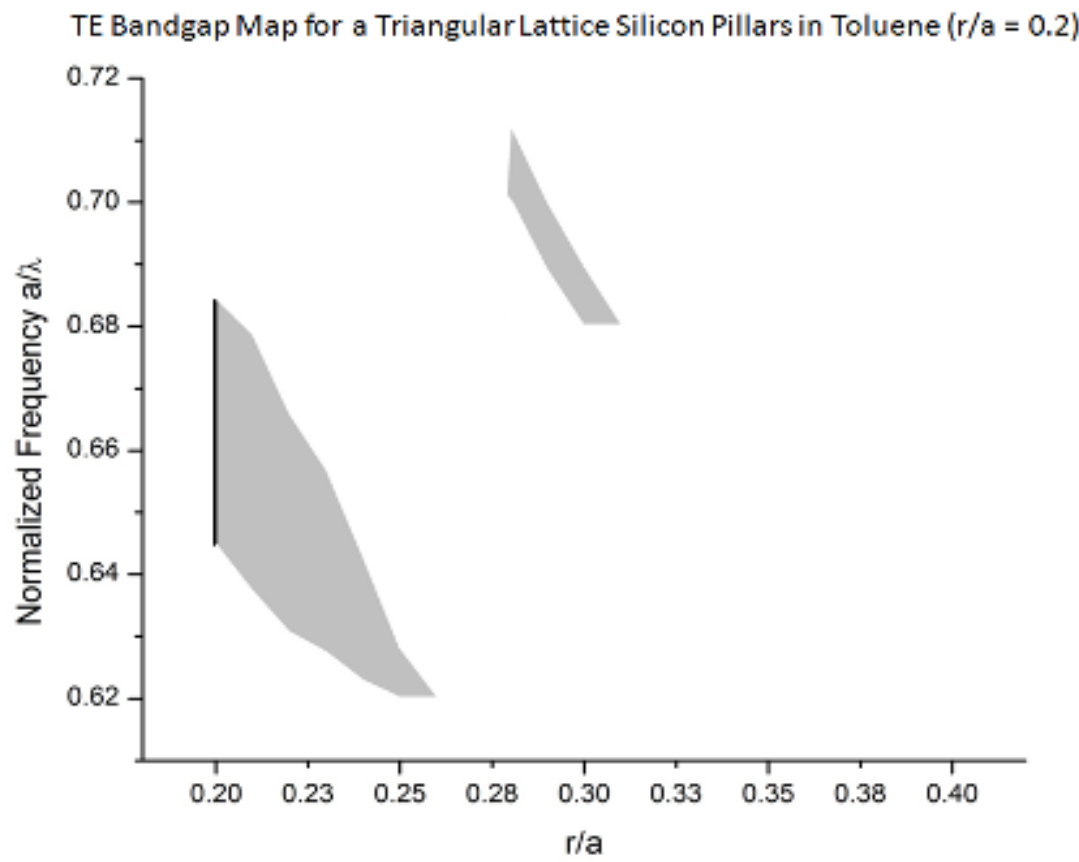

Figure 3.12. TE bandgap map for a triangular lattice of silicon pillars surrounded by toluene. The widest observed bandgap for this lattice was found at $\mathrm{r} / \mathrm{a}=0.2$ 
TM Band Diagram of a Triangular Lattice of Silicon Pillars in Toluene $(r / a=0.22 a)$

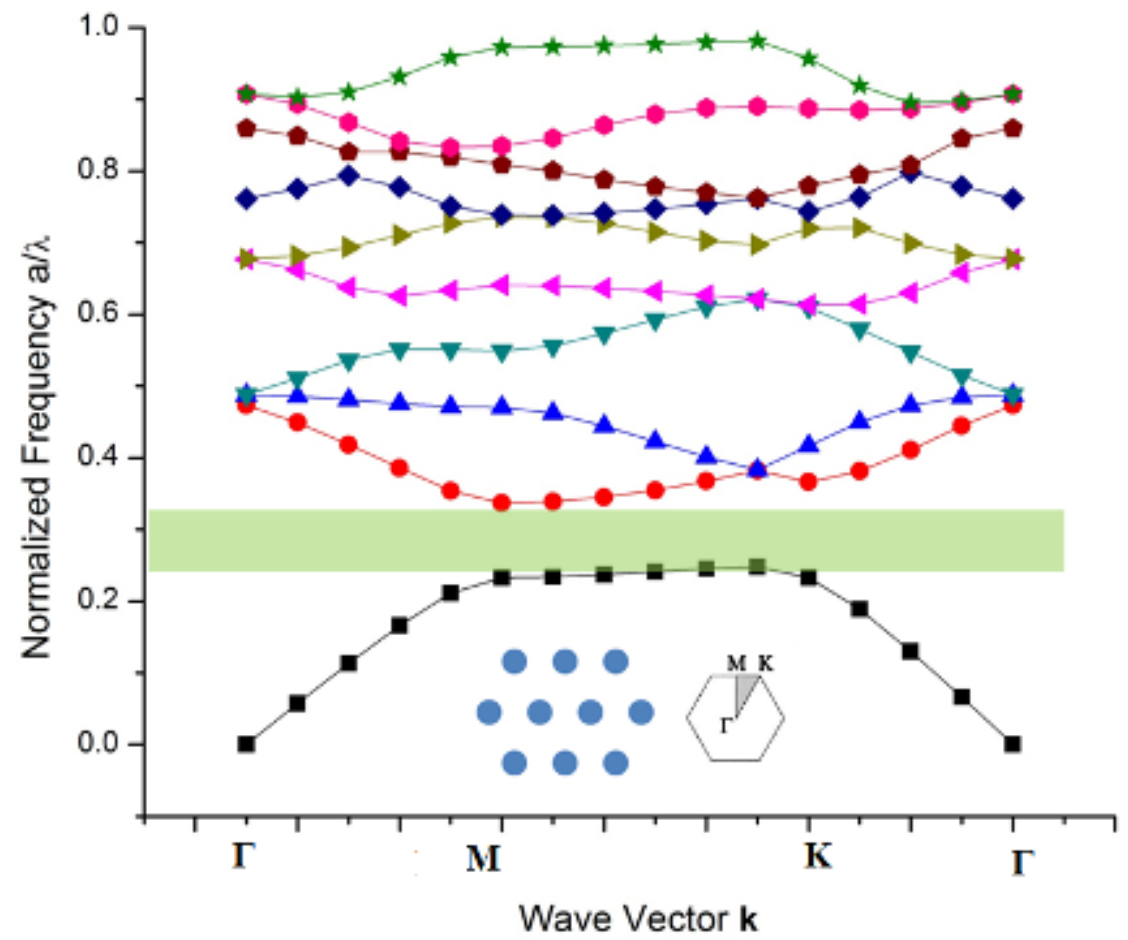

Figure 3.13. TM Band diagram of a triangular lattice of silicon pillars in toluene with r/a $=0.2$

TE Band Diagram of a Triangular Lattice of Silicon Pillars in Toluene $(r=0.20 \mathrm{a})$

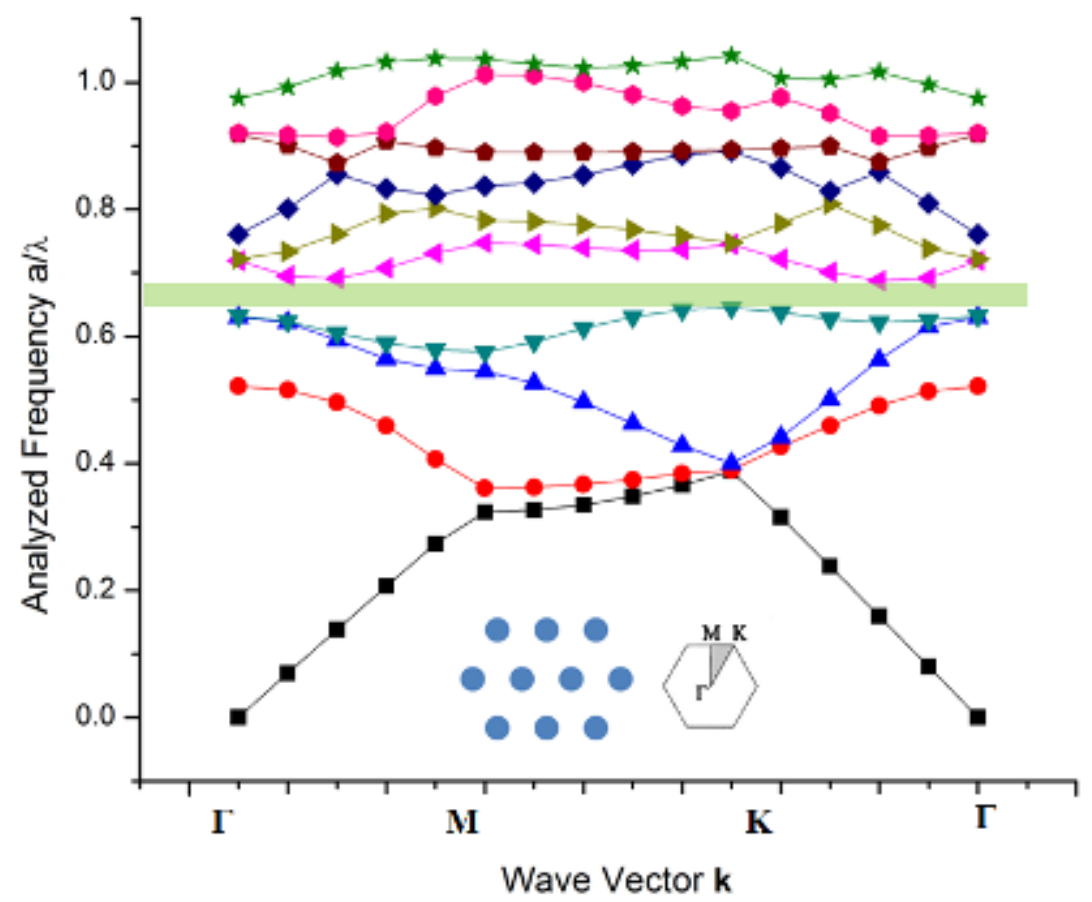

Figure 3.14. TE band diagram of the largest TE bandgap between the $4^{\text {th }}$ and $5^{\text {th }}$ bands for a triangular lattice of silicon pillars surrounded by toluene with $\mathrm{r} / \mathrm{a}=0.2$ 
Tables 3.1-3.4 demonstrate detailed information regarding the bandgap size for each of the previous lattices as well as the range of frequencies of the bandgaps.

Table 3.1. Summarized modeling results for the lattice of a square lattice of toluene holes in silicon

\begin{tabular}{|c|c|c|c|c|c|c|c|c|}
\hline \multicolumn{9}{|c|}{ Square Lattice of Toluene Holes in Silicon } \\
\hline \multicolumn{9}{|c|}{ Si_Eps=12.5036 Toluene_Eps=2.24109 } \\
\hline $\mathrm{r} / \mathrm{a}$ & \multicolumn{4}{|c|}{ TM } & \multicolumn{4}{|c|}{$\mathrm{TE}$} \\
\hline & Bands & bottom & top & Bandgap Width (\%) & Bands & bottom & top & Bandgap Width (\%) \\
\hline 0.2 & $\mathrm{~N} / \mathrm{A}$ & & & & $\mathrm{N} / \mathrm{A}$ & & & \\
\hline 0.21 & N/A & & & & N/A & & & \\
\hline 0.22 & N/A & & & & N/A & & & \\
\hline 0.23 & N/A & & & & N/A & & & \\
\hline 0.24 & N/A & & & & N/A & & & \\
\hline 0.25 & $\mathrm{~N} / \mathrm{A}$ & & & & N/A & & & \\
\hline 0.26 & $\mathrm{~N} / \mathrm{A}$ & & & & N/A & & & \\
\hline 0.27 & N/A & & & & N/A & & & \\
\hline 0.28 & $\mathrm{~N} / \mathrm{A}$ & & & & N/A & & & \\
\hline 0.29 & N/A & & & & N/A & & & \\
\hline 0.3 & N/A & & & & N/A & & & \\
\hline 0.31 & N/A & & & & N/A & & & \\
\hline 0.32 & N/A & & & & N/A & & & \\
\hline 0.33 & N/A & & & & N/A & & & \\
\hline 0.34 & $\mathrm{~N} / \mathrm{A}$ & & & & N/A & & & \\
\hline 0.35 & 8and9 & 0.538 & 0.546198 & 1.50129 & N/A & & & \\
\hline 0.36 & $\mathrm{~N} / \mathrm{A}$ & & & & N/A & & & \\
\hline 0.37 & N/A & & & & N/A & & & \\
\hline 0.38 & $\mathrm{~N} / \mathrm{A}$ & & & & N/A & & & \\
\hline 0.39 & $\mathrm{~N} / \mathrm{A}$ & & & & N/A & & & \\
\hline 0.4 & $\mathrm{~N} / \mathrm{A}$ & & & & N/A & & & \\
\hline
\end{tabular}


Table 3.2. Summarized modeling results for the lattice of silicon pillars in toluene

\begin{tabular}{|c|c|c|c|c|c|c|c|c|c|}
\hline \multicolumn{10}{|c|}{ Square Lattice of Silicon Pillars in Toluene } \\
\hline \multicolumn{10}{|c|}{ Si_Eps=12.5036 Toluene_Eps=2.24109 } \\
\hline \multirow[t]{2}{*}{$r / a$} & \multicolumn{4}{|c|}{ TM } & \multicolumn{4}{|c|}{ TE } & \\
\hline & Bands & bottom & top & $\begin{array}{c}\text { Bandgap } \\
\text { Width (\%) }\end{array}$ & Bands & bottom & top & $\begin{array}{c}\text { Bandgap } \\
\text { Width (\%) }\end{array}$ & \\
\hline \multirow[t]{2}{*}{0.2} & 1and2 & 0.266183 & 0.31385 & 16.201 & $\mathrm{~N} / \mathrm{A}$ & & & & \\
\hline & 4and5 & 0.579826 & 0.589913 & 1.72477 & $\mathrm{~N} / \mathrm{A}$ & & & & \\
\hline \multirow[t]{2}{*}{0.21} & 1and2 & 0.259163 & 0.308643 & 17.4284 & 4and5 & 0.617409 & 0.623066 & 0.912016 & \\
\hline & 4and5 & 0.573098 & 0.576097 & 0.521958 & 9and10 & 0.916027 & 0.92954 & 1.46495 & \\
\hline \multirow[t]{2}{*}{0.22} & 1and2 & 0.254084 & 0.304445 & 18.033669 & 4and5 & 0.60739 & 0.614736 & 1.20067 & \\
\hline & & & & & 9and10 & 0.898868 & 0.920398 & 2.36688 & \\
\hline \multirow[t]{2}{*}{0.23} & 1and2 & 0.250442 & 0.301016 & 18.341879 & 4and 5 & 0.60047 & 0.609805 & 1.54139 & \\
\hline & & & & & 9and10 & 0.8835 & 0.913702 & 3.3609 & \\
\hline \multirow[t]{2}{*}{0.24} & 1and2 & 0.243862 & 0.293771 & 18.566371 & 4and5 & 0.58448 & 0.599213 & 2.488984 & \\
\hline & & & & & 9and10 & 0.850177 & 0.894461 & 5.076521 & \\
\hline \multirow[t]{2}{*}{0.25} & 1and2 & 0.240131 & 0.289018 & 18.47721 & 4and5 & 0.57444 & 0.586376 & 2.0549617 & \\
\hline & 4and5 & 0.547848 & 0.552618 & 0.86705 & 9and10 & 0.83985 & 0.880414 & 4.715299 & \\
\hline \multirow[t]{2}{*}{0.26} & 1and2 & 0.236485 & 0.283859 & 18.208542 & 4and5 & 0.565709 & 0.579929 & 2.482338 & \\
\hline & 4and5 & 0.542883 & 0.545721 & 0.521389 & 9and10 & 0.832681 & 0.865229 & 3.833866 & \\
\hline \multirow[t]{2}{*}{0.27} & 1and2 & 0.23208 & 0.276875 & 17.60266 & 4and5 & 0.553278 & 0.56936 & 2.864903 & \\
\hline & 3and4 & 0.417856 & 0.431847 & 3.29323 & 9and10 & 0.823432 & 0.839236 & 1.90118 & \\
\hline \multirow[t]{2}{*}{0.28} & 1and2 & 0.229216 & 0.271887 & 17.03089 & 4and5 & 0.544786 & 0.562308 & 3.16528 & \\
\hline & 3and4 & 0.409355 & 0.431426 & 5.250082 & 9and10 & 0.814902 & 0.826424 & 1.40398 & \\
\hline \multirow[t]{2}{*}{0.29} & 1and2 & 0.226299 & 0.266356 & 16.261509 & 4and5 & 0.534837 & 0.550712 & 2.92474 & \\
\hline & 3and4 & 0.400675 & 0.430693 & 7.221346 & 9and10 & 0.805947 & 0.807679 & 0.214659 & \\
\hline \multirow[t]{2}{*}{0.3} & 1and2 & 0.223771 & 0.261144 & 15.414543 & 4and5 & 0.525517 & 0.545187 & 3.674361 & \\
\hline & 3and4 & 0.393123 & 0.427719 & 8.42957 & & & & & \\
\hline \multirow[t]{2}{*}{0.31} & 1and2 & 0.220937 & 0.254932 & 14.287791 & 4and5 & 0.515277 & 0.533876 & 3.54555 & \\
\hline & 3and4 & 0.384623 & 0.419656 & 8.711678 & & & & & \\
\hline \multirow[t]{2}{*}{0.32} & 1and2 & 0.21844 & 0.248975 & 13.065367 & 4and5 & 0.505463 & 0.521381 & 3.100426 & \\
\hline & 3and 4 & 0.377075 & 0.411009 & 8.61154 & & & & & \\
\hline \multirow[t]{2}{*}{0.33} & 1and2 & 0.216132 & 0.242942 & 11.680195 & 4and5 & 0.49563 & 0.511406 & 3.13329 & \\
\hline & 3and4 & 0.370042 & 0.402192 & 8.32654 & & & & & \\
\hline \multirow[t]{2}{*}{0.34} & 1and2 & 0.214207 & 0.237655 & 10.378462 & 4and5 & 0.486346 & 0.501221 & 3.012436 & \\
\hline & 3and4 & 0.36414 & 0.393935 & 7.858907 & & & & & \\
\hline \multirow[t]{2}{*}{0.35} & 1and2 & 0.212338 & 0.232067 & 8.878968 & 4and5 & 0.477416 & 0.492525 & 3.11549 & \\
\hline & 3and4 & 0.358361 & 0.384924 & 7.147452 & & & & & \\
\hline 0.36 & 1and2 & 0.210566 & 0.226314 & 7.20944 & 4and5 & 0.466899 & 0.478336 & 2.419912 & \\
\hline & 3and4 & 0.352796 & 0.374279 & 5.909492 & 6and7 & 0.58739 & 0.595339 & 1.343331 & \\
\hline 0.37 & 1and2 & 0.20922 & 0.221618 & 5.755349 & 4and5 & 0.458999 & 0.473003 & 3.00523 & \\
\hline & 3and4 & 0.348573 & 0.36754 & 5.299134 & 6and7 & 0.577412 & 0.58613 & 1.498433 & \\
\hline 0.38 & 1and2 & 0.207738 & 0.216063 & 3.928922 & 4and5 & 0.450229 & 0.460606 & 2.278535 & \\
\hline & 3and4 & 0.343822 & 0.357206 & 3.818557 & 6and7 & 0.563967 & 0.575427 & 2.011651 & \\
\hline 0.39 & 1and2 & 0.206799 & 0.212267 & 2.6096 & 4and5 & 0.445169 & 0.451897 & 1.499959 & \\
\hline & 3and 4 & 0.340756 & 0.349414 & 2.50872 & 6and7 & 0.554024 & 0.567556 & 2.4129322 & \\
\hline 0.4 & 1and2 & 0.205428 & 0.205986 & 0.2712369 & 4and5 & 0.436745 & 0.439588 & 0.648997 & \\
\hline & 3and4 & 0.336294 & 0.339974 & 1.088481 & 6and7 & 0.540916 & 0.550746 & 1.80087 & \\
\hline
\end{tabular}


Table 3.3. Summarized simulation results of the bandgap for both polarizations of a triangular lattice of toluene holes in silicon

\begin{tabular}{|c|c|c|c|c|c|c|c|c|}
\hline \multicolumn{7}{|c|}{ Triangular Lattice of Toluene Holes in Silicon } \\
\hline \multicolumn{9}{|c|}{ Si_Eps=12.5036 Toluene_Eps=2.24109 } \\
\hline r/a & \multicolumn{7}{|c|}{} & \multicolumn{3}{|c|}{ TE } \\
\hline & Bands & bottom & top & Bandgap Width (\%) & Bands & bottom & top & Bandgap Width (\%) \\
\hline 0.2 & N/A & & & & 1and2 & 0.191201 & 0.196046 & 2.502657 \\
\hline 0.21 & N/A & & & & 1and2 & 0.191675 & 0.199226 & 3.863429 \\
\hline 0.22 & N/A & & & & 1and2 & 0.192325 & 0.203213 & 5.503 \\
\hline 0.23 & N/A & & & & 1and2 & 0.192857 & 0.206195 & 6.684539 \\
\hline 0.24 & N/A & & & & 1and2 & 0.193771 & 0.211155 & 8.58631 \\
\hline 0.25 & 6and7 & 0.4614 & 0.46439 & 0.6294 & 1and2 & 0.194732 & 0.215951 & 10.33362 \\
\hline 0.26 & 6and7 & 0.4655 & 0.4694 & 0.88115 & 1and2 & 0.195799 & 0.220836 & 12.01871 \\
\hline 0.27 & 6and7 & 0.46938 & 0.47223 & 0.60632 & 1and2 & 0.196779 & 0.225175 & 13.45905 \\
\hline 0.28 & 6and7 & 0.474238 & 0.475944 & 0.35912 & 1and2 & 0.198096 & 0.230722 & 15.21691 \\
\hline 0.29 & 6and7 & 0.4801 & 0.4814 & 0.266 & 1and2 & 0.199978 & 0.237929 & 17.33297 \\
\hline 0.3 & 6and7 & 0.4848 & 0.4862 & 0.299022 & 1and2 & 0.201445 & 0.243368 & 18.84992 \\
\hline 0.31 & 6and7 & 0.48919 & 0.49023 & 0.213 & 1and2 & 0.202844 & 0.24801 & 20.03573 \\
\hline 0.32 & 6and7 & 0.4951 & 0.498 & 0.58436 & 1and2 & 0.205147 & 0.25541 & 21.82697 \\
\hline 0.33 & 6and7 & 0.50237 & 0.50597 & 0.71554 & 1and2 & 0.207628 & 0.26281 & 23.45985 \\
\hline 0.34 & 6and7 & 0.5106 & 0.512 & 0.2752 & 1and2 & 0.210136 & 0.26977 & 24.85557 \\
\hline 0.35 & 6and7 & 0.51817 & 0.51911 & 0.182 & 1and2 & 0.212751 & 0.275989 & 25.87793 \\
\hline 0.36 & 6and7 & 0.52633 & 0.52981 & 0.65877 & 1and2 & 0.216038 & 0.283998 & 27.18188 \\
\hline 0.37 & 6and7 & 0.53614 & 0.542693 & 1.214639 & 1and2 & 0.220322 & 0.293096 & 28.34865 \\
\hline 0.38 & 6and7 & 0.54479 & 0.55253 & 1.4106 & 1and2 & 0.223835 & 0.299493 & 28.91405 \\
\hline & 7and8 & 0.60143 & 0.60698 & 0.91855 & & & & \\
\hline 0.39 & 6and7 & 0.555407 & 0.562184 & 1.212787 & 1and2 & 0.227694 & 0.306133 & 29.38733 \\
\hline & 7and8 & 0.610619 & 0.625204 & 2.434075 & & & & \\
\hline 0.4 & 6and7 & 0.572156 & 0.575489 & 0.580822 & 1and2 & 0.233588 & 0.31471 & 29.59048 \\
\hline & 7and8 & 0.622462 & 0.631943 & 1.51155 & & & & \\
\hline
\end{tabular}


Table 3.4. Summarized simulation results of the bandgap for both polarizations of a triangular lattice of silicon pillars in toluene

\begin{tabular}{|c|c|c|c|c|c|c|c|c|}
\hline \multicolumn{9}{|c|}{ Triangular Lattice of Silicon Pillars in Toluene } \\
\hline \multicolumn{9}{|c|}{ Si_Eps $=12.5036$ Toluene_Eps $=2.24109$} \\
\hline \multirow[t]{2}{*}{$\mathrm{r} / \mathrm{a}$} & \multicolumn{4}{|c|}{ TM } & \multicolumn{4}{|c|}{ TE } \\
\hline & Bands & bottom & top & Bandgap Width (\%) & Bands & bottom & top & Bandgap Width (\%) \\
\hline 0.2 & 1and2 & 0.258599 & 0.350124 & 30.0711 & 4and5 & 0.645221 & 0.684552 & 6.35225 \\
\hline 0.21 & 1and2 & 0.253279 & 0.344029 & 30.38629 & 4and5 & 0.637625 & 0.678736 & 6.24615 \\
\hline 0.22 & 1and2 & 0.247513 & 0.337004 & 30.6204 & 4and5 & 0.631007 & 0.665944 & 5.387637 \\
\hline \multirow[t]{2}{*}{0.23} & 1and2 & 0.243852 & 0.331955 & 30.6015 & 4and5 & 0.627753 & 0.656859 & 4.531557 \\
\hline & & & & & 9and10 & 0.940269 & 0.949892 & 1.018237 \\
\hline \multirow[t]{2}{*}{0.24} & 1and2 & 0.238182 & 0.322969 & 30.21606 & 4and5 & 0.623176 & 0.642779 & 3.09701 \\
\hline & 3and4 & 0.46052 & 0.472789 & 2.629218 & & & & \\
\hline \multirow[t]{2}{*}{0.25} & 1and2 & 0.233604 & 0.315312 & 29.7706 & 4and5 & 0.620423 & 0.628213 & 1.247668 \\
\hline & 3and4 & 0.44694 & 0.47242 & 5.543039 & & & & \\
\hline \multirow[t]{2}{*}{0.26} & 1and2 & 0.229625 & 0.30766 & 29.04771 & $\mathrm{~N} / \mathrm{A}$ & & & \\
\hline & 3and4 & 0.434887 & 0.471649 & 8.110488 & & & & \\
\hline \multirow[t]{2}{*}{0.27} & 1and2 & 0.226349 & 0.301005 & 28.31307 & $\mathrm{~N} / \mathrm{A}$ & & & \\
\hline & 3and4 & 0.425661 & 0.470626 & 10.0335 & & & & \\
\hline \multirow[t]{2}{*}{0.28} & 1and2 & 0.222796 & 0.293628 & 27.43194 & 6and7 & 0.701046 & 0.712512 & 1.62262 \\
\hline & 3and4 & 0.415145 & 0.468773 & 12.13397 & & & & \\
\hline \multirow[t]{3}{*}{0.29} & 1and2 & 0.218838 & 0.284269 & 26.01079 & 6and7 & 0.689554 & 0.700188 & 1.530691 \\
\hline & 3and4 & 0.403498 & 0.465434 & 14.25568 & & & & \\
\hline & 6and7 & 0.60925 & 0.628147 & 3.054344 & & & & \\
\hline \multirow[t]{3}{*}{0.3} & 1and2 & 0.216164 & 0.27781 & 24.95903 & 6and7 & 0.680473 & 0.6898 & 1.374627 \\
\hline & 3and4 & 0.395542 & 0.462277 & 15.55927 & & & & \\
\hline & 6and7 & 0.59665 & 0.626652 & 4.905006 & & & & \\
\hline \multirow[t]{3}{*}{0.31} & 1and2 & 0.214155 & 0.272729 & 24.06091 & N/A & & & \\
\hline & 3and4 & 0.389998 & 0.459282 & 16.31588 & & & & \\
\hline & 6and7 & 0.587044 & 0.625064 & 6.27346 & & & & \\
\hline \multirow[t]{3}{*}{0.32} & 1and2 & 0.211281 & 0.264679 & 22.43797 & $\mathrm{~N} / \mathrm{A}$ & & & \\
\hline & 3and4 & 0.381755 & 0.453924 & 17.27187 & & & & \\
\hline & 6and7 & 0.571702 & 0.620824 & 8.23835 & & & & \\
\hline \multirow[t]{3}{*}{0.33} & 1and2 & 0.208776 & 0.25769 & 20.97185 & $\mathrm{~N} / \mathrm{A}$ & & & \\
\hline & 3and4 & 0.374321 & 0.444036 & 17.03779 & & & & \\
\hline & 6and7 & 0.559392 & 0.615384 & 9.53253 & & & & \\
\hline \multirow[t]{3}{*}{0.34} & 1and2 & 0.206766 & 0.251563 & 19.54781 & $\mathrm{~N} / \mathrm{A}$ & & & \\
\hline & 3and4 & 0.368576 & 0.433508 & 16.19075 & & & & \\
\hline & 6and7 & 0.548763 & 0.599085 & 8.768008 & & & & \\
\hline 0.35 & 1and2 & 0.204984 & 0.245765 & 18.09446 & N/A & & & \\
\hline & 3and4 & 0.363849 & 0.424077 & 15.28765 & & & & \\
\hline & 6and7 & 0.538937 & 7.944803 & 7.944803 & & & & \\
\hline 0.36 & 1and2 & 0.203106 & 0.239675 & 16.51801 & $\mathrm{~N} / \mathrm{A}$ & & & \\
\hline & 3and4 & 0.358359 & 0.413316 & 14.24327 & & & & \\
\hline & 6and7 & 0.529298 & 0.567046 & 6.886245 & & & & \\
\hline 0.37 & 1and2 & 0.201167 & 0.232677 & 14.52603 & N/A & & & \\
\hline & 3and4 & 0.353063 & 0.401372 & 12.80687 & & & & \\
\hline & 6and7 & 0.518226 & 0.546727 & 5.333937 & & & & \\
\hline 0.38 & 1and2 & 0.199885 & 0.227847 & 13.07479 & $\mathrm{~N} / \mathrm{A}$ & & & \\
\hline & 3and4 & 0.349615 & 0.393234 & 11.74369 & & & & \\
\hline & 6and7 & 0.511226 & 0.533616 & 4.285838 & & & & \\
\hline 0.39 & 1and2 & 0.198716 & 0.223416 & 11.70253 & N/A & & & \\
\hline & 3and4 & 0.346505 & 0.385304 & 10.60355 & & & & \\
\hline & 6and7 & 0.50508 & 0.522527 & 3.39551 & & & & \\
\hline 0.4 & 1and2 & 0.19725 & 0.217087 & 9.575288 & $\mathrm{~N} / \mathrm{A}$ & & & \\
\hline & 3and4 & 0.342697 & 0.37488 & 8.969976 & & & & \\
\hline & 6and7 & 0.496327 & 0.503175 & 1.370387 & & & & \\
\hline
\end{tabular}




\subsubsection{Analyzing Infinitely-Thick Photonic Crystal Lattices of Silicon and Toluene}

In the previous section, we considered different lattices of silicon and toluene as the high and low dielectric region within their lattices, respectively. The band structure of silicon pillars in toluene of both triangular and square lattices were demonstrated in Figures 3.4-3.5 and 3.12-3.13. The horizontal axis demonstrates the value of the in-plane wave vector that is moved along the edges of the irreducible Brillouin zone. Those edges are selected because the minima and maxima frequency points of a given bandgap almost always occur at these edges. In the next section, however, it will be demonstrated that this is not the always the case when considering slab photonic crystals sandwiched between low dielectric regions other than air.

For the square and triangular lattices of silicon pillars surrounded by toluene background, it can be clearly noticed that a complete and wide TM bandgaps were resolved. In contrast, for the TE modes there were very narrow bandgaps. The reason for this is that the field associated with the lowest TM modes of the dielectric band (or low frequency bands) is very highly concentrated in the silicon pillars. On the other hand, the field pattern of the air bands (high frequency bands) is highly concentrated in the toluene regions surrounding the silicon pillars. This contrast in field concentrations of consecutive TM modes leads to the observed wide TM photonic bandgaps. Alternatively, the concentration contrast between the electric field of the high and low consecutive TE modes in the high and low dielectric regions is not as strong as that for the TM polarization modes. In fact, the TE modes in these lattices were forced to penetrate the toluene regions as the field lines cross the dielectric boundaries. This is the reason why wide TE bandgaps for these lattices were not observed.

The other lattices of toluene holes in silicon presented a more dispersed distribution of high dielectric material, and hence, it allowed the TE field lines to propagate along these regions. Therefore, the opposite applies to these lattices and wide TE bandgaps were resolved instead of TM.

Since complete photonic bandgaps for both polarizations were not observed in any of the studied lattices, those lattices that presented wide bandgaps in either polarizations are going to be studied further when slab photonic crystals are considered. These lattices were:

- Square lattice of silicon pillars surrounded by toluene with $\mathrm{r} / \mathrm{a}=0.24$

- Triangular lattice of toluene holes in silicon with $\mathrm{r} / \mathrm{a}=0.35$

- Triangular lattice of silicon pillars surrounded by toluene with $\mathrm{r} / \mathrm{a}=0.22$

The dimensions of each lattice to be considered for fabrication are explained in tables 3.5-3.8. 
Table 3.5. Actual lattice parameters for a square lattice of infinitely-high silicon pillars surrounded by toluene

\begin{tabular}{|c|c|c|c|c|c|c|}
\hline \multicolumn{5}{|c|}{ Square Lattice of Silicon Pillars Surrounded by Toluene } \\
\hline $\mathrm{r} / \mathrm{a}$ & Gap bottom frequency $(\mathrm{a} / \lambda)$ & Gap Top Frequency $(\mathrm{a} / \lambda)$ & Mid Gap $(\mathrm{a} / \lambda)$ & Actual Lattice Constant $(\mathrm{a})=1100 \mathrm{~nm} *$ Mid Gap & Actual Radius $(\mathrm{r})=\mathrm{a} * 0.24$ \\
\hline 0.24 & 0.243862 & 0.293771 & 0.2688165 & $295.69815 \mathrm{~nm}$ & $70.967556 \mathrm{~nm}$ \\
\hline
\end{tabular}

Table 3.6. Actual lattice parameters for a triangular lattice of infinitely-thick toluene holes in silicon

\begin{tabular}{|c|c|c|c|c|c|}
\hline \multicolumn{5}{|c|}{ Triangular Lattice of Air Holes in Silicon } \\
\hline $\mathrm{r} / \mathrm{a}$ & Gap bottom frequency $(\mathrm{a} / \mathrm{\lambda})$ & Gap Top Frequency $(\mathrm{a} / \lambda)$ & Mid Gap $(\mathrm{a} / \lambda)$ & Actual Lattice Constant $(\mathrm{a})=1100 \mathrm{~nm} * \mathrm{Mid}$ Gap & Actual Radius $(\mathrm{r})=\mathrm{a} * 0.35$ \\
\hline 0.35 & 0.212751 & 0.275989 & 0.24437 & $268.807 \mathrm{~nm}$ & $94.08245 \mathrm{~nm}$ \\
\hline
\end{tabular}

Table 3.7. Actual lattice parameters for a triangular lattice of silicon pillars surrounded by toluene

\begin{tabular}{|c|c|c|c|c|c|}
\hline \multicolumn{5}{|c|}{ Triangular Lattice of Sillicon Pillars Surrounded by Toluene } \\
\hline $\mathrm{r} / \mathrm{a}$ & Gap bottom frequency $(\mathrm{a} / \lambda)$ & Gap Top Frequency $(\mathrm{a} / \lambda)$ & Mid Gap $(\mathrm{a} / \lambda)$ & Actual Lattice Constant $(\mathrm{a})=1100 \mathrm{~nm} *$ Mid Gap & Actual Radius $(\mathrm{r})=\mathrm{a} * 0.35$ \\
\hline 0.22 & 0.247513 & 0.337004 & 0.2922585 & $321.48435 \mathrm{~nm}$ & $70.726557 \mathrm{~nm}$ \\
\hline
\end{tabular}




\subsubsection{Finite-Thickness Slab Photonic Crystals of Silicon, Toluene, and Air}

In the previous section, we started by modeling infinitely thick photonic crystal lattices to have an idea of where the photonic bandgaps for each lattice can be found. If an infinitely-thick photonic crystal lattice demonstrated no bandgap or very narrow bandgaps in either polarization, then this lattice will mostly not posses any bandgap effects when a finite thickness is introduced in the vertical dimension. Further, published results indicate that moving from an infinitely thick photonic crystals to slab photonic crystals causes a shrinkage of the bandgap by at least 40\% [47-49].

Two-dimensional photonic crystal slabs (Figure 2.7) are similar to infinitely-thick photonic crystals in their in-plane periodicity. However, two-dimensional calculations cannot be applied directly to finitethickness slab structures. Moving from an infinitely long structure to one with a finite thickness recreates the band gap in the guided slab modes. The modeled material system then becomes fundamentally threedimensional and distinct from the two dimensional calculations with a new set of parameters that must be considered such as: slab thickness, effective refractive index contrast of the slab and the background above and below the slab, and mirror symmetry of the photonic crystal slab in the vertical direction [46].

When modeling photonic crystal slabs for practical applications, it is also very important to consider the effective refractive index of the background medium above and below the slab because, in most applications (specifically in our proposed transducer as will be discussed in the following chapter) this medium can be air, water, or any other solution. However, since the PbS Quantum Dots (QDs) are suspended in toluene when characterizing the photonic crystal structures, the low dielectric regions are assumed to have the refractive index of toluene.

The photonic crystal parameters that were extracted from modeling infinitely-thick photonic crystals were used when modeling the finite-thickness slabs. Specifically, the main interest is on the three lattices that demonstrated the widest bandgaps. These lattices are:

- Square lattice of silicon pillars surrounded by toluene with $\mathrm{r} / \mathrm{a}=0.24$

- Triangular lattice of toluene holes in silicon with $\mathrm{r} / \mathrm{a}=0.35$

- Triangular lattice of silicon pillars surrounded by toluene with $\mathrm{r} / \mathrm{a}=0.22$

For each lattice, the slab thickness was varied between $h=0.5 \mathrm{a}$ to $\mathrm{h}=1.2 \mathrm{a}$ in increments of 0.1 to determine whether these slabs posses a bandgap or not. However, it is also important to point out that some finite-thickness slabs, i.e. lattices of silicon pillars in air, are not realistic for our application. For example, a triangular lattice of silicon pillars surrounded by toluene cannot be free standing when 'sandwiched' by toluene. Therefore, $\mathrm{SiO}_{2}$ was chosen to be the material below these slabs. When fabricated, the photonic crystal can either be made in the silicon layer above the silicon dioxide layer or extruded to the $\mathrm{SiO}_{2}$ layer below it. Very minor modifications to the band diagram are expected to be 
observed since the dielectric constants of toluene and $\mathrm{SiO}_{2}$ are very close to each other. On the other hand, those lattices of toluene holes in silicon can indeed be fabricated to be free-standing structures sandwiched between toluene. Figures 3.15-3.22 demonstrate the band diagrams of the TE- and TM-like polarizations for square and triangular lattices of silicon pillars lattices surrounded by toluene and sandwiched in between two $\mathrm{SiO}_{2}$ layers. The band diagrams are shown for those specific lattices with the widest bandgap in their infinitely-thick representation. The results presented here are summarized for the thinnest and thickest slabs in the range between $0.5 a$ and $1.2 a$.

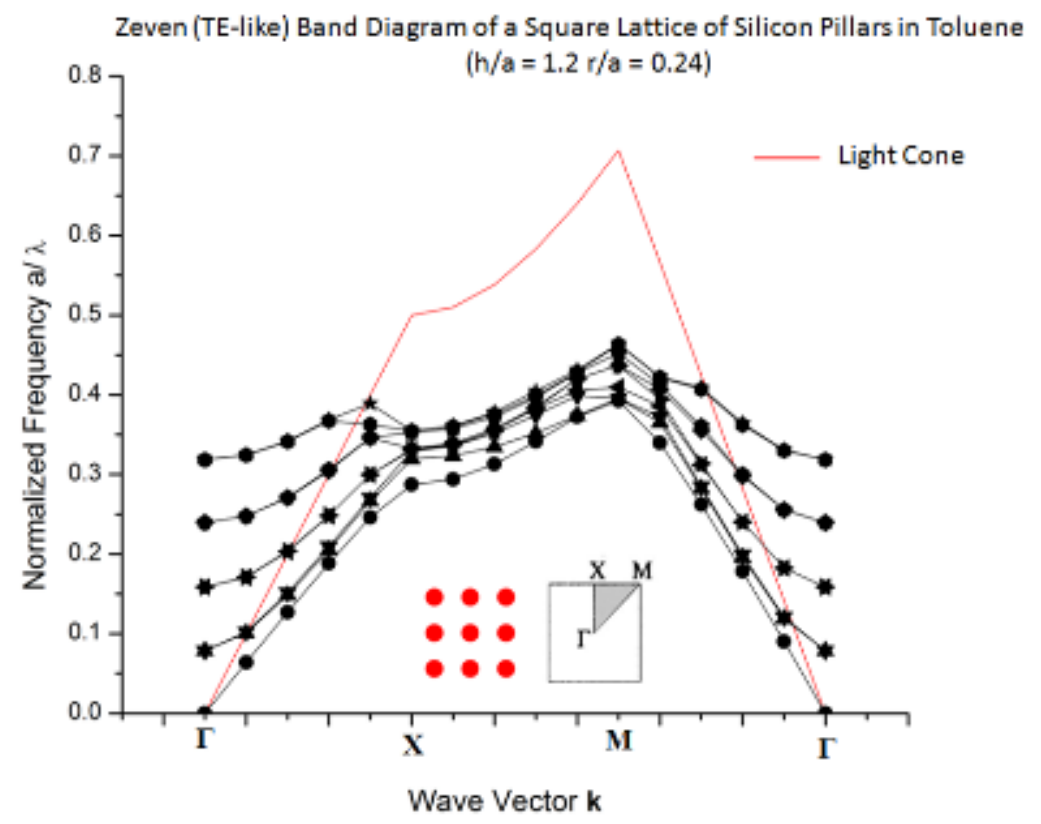

Figure 3.15. TE-like band diagram for a square lattice of Si pillars in Toluene demonstrating no bandgap within the guided modes below the light cone can be observed. 


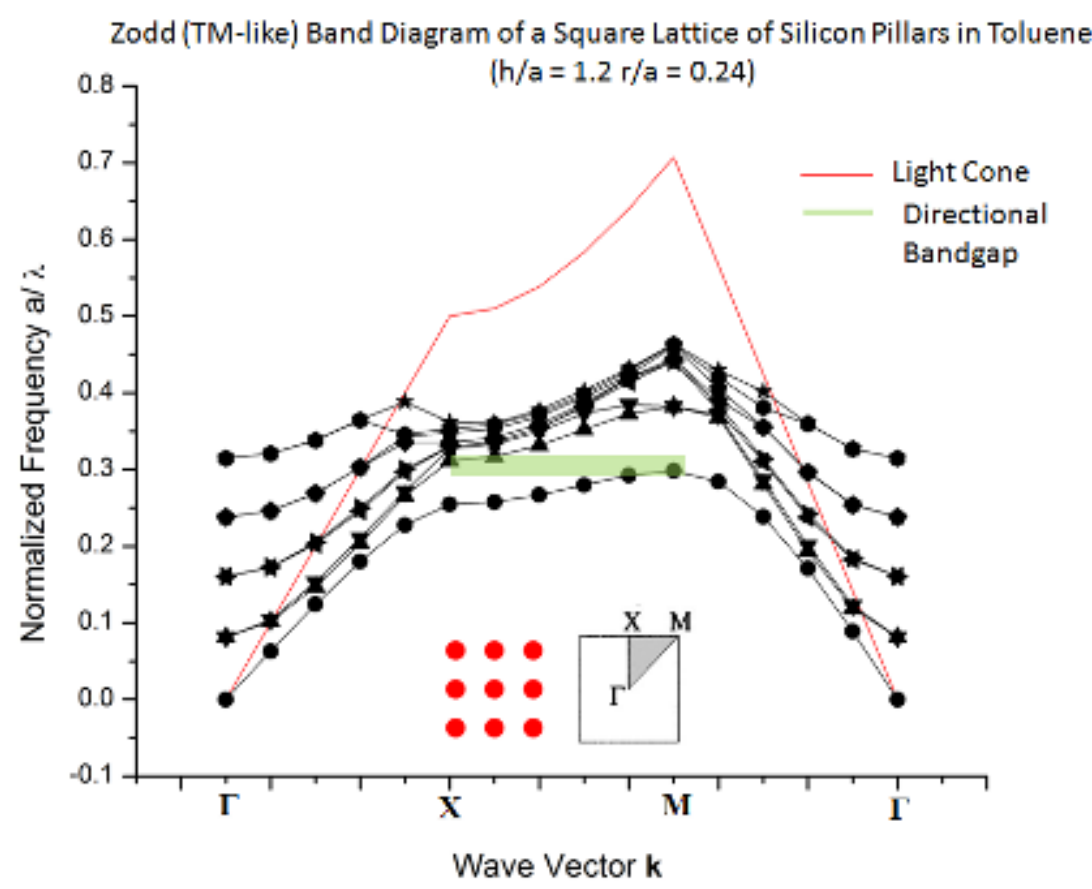

Figure 3.16. Zodd (TM-like) band diagram of a slab photonic crystal $(\mathrm{h}=1.2 a)$ square lattice of silicon pillars immersed in toluene. Only a directional bandgap can be observed between the $\mathrm{X}$ and $\mathrm{M}$ points.

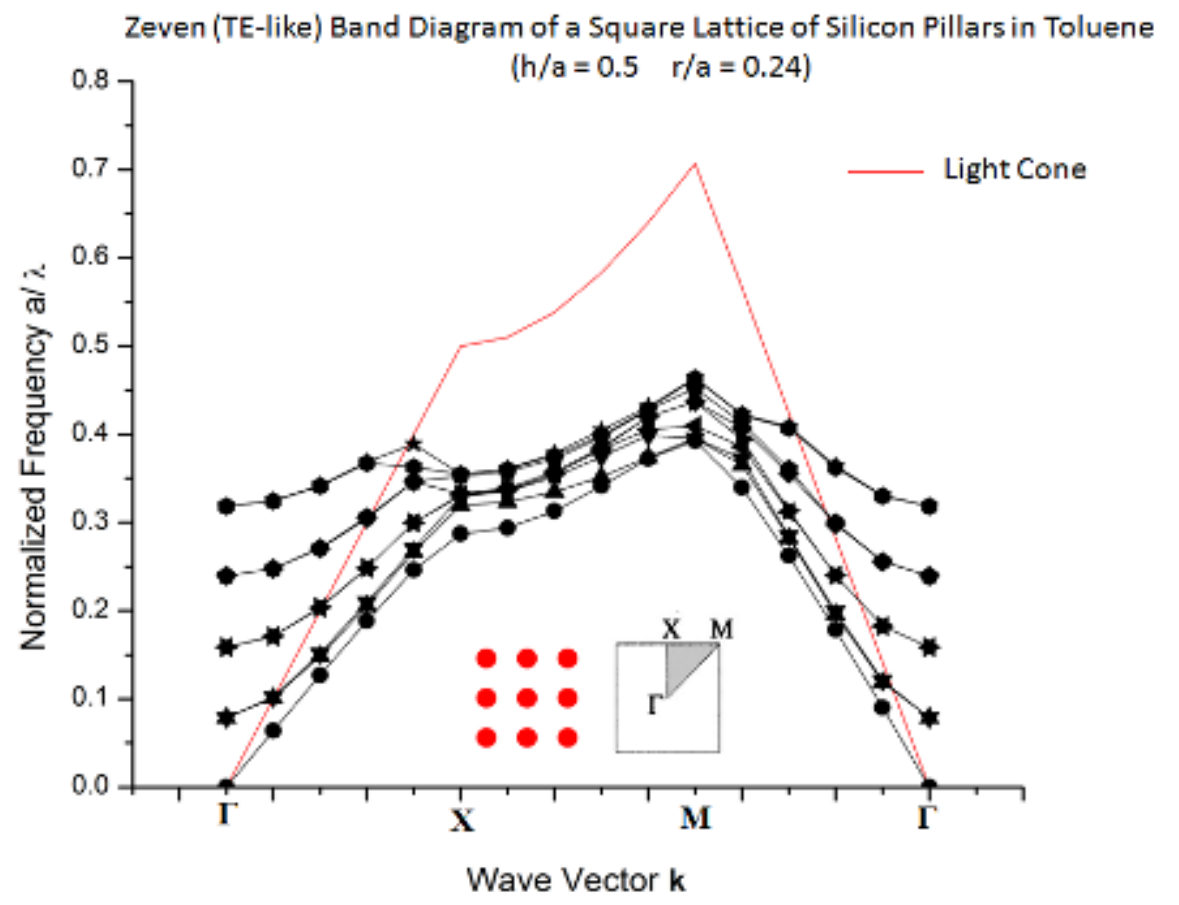

Figure 3.17. Zeven (TE-like) band diagram of a slab photonic crystal square lattice $(\mathrm{h}=0.5 a$ ) of silicon pillars in toluene. No bandgap can be resolved under the light cone for the guided modes. 


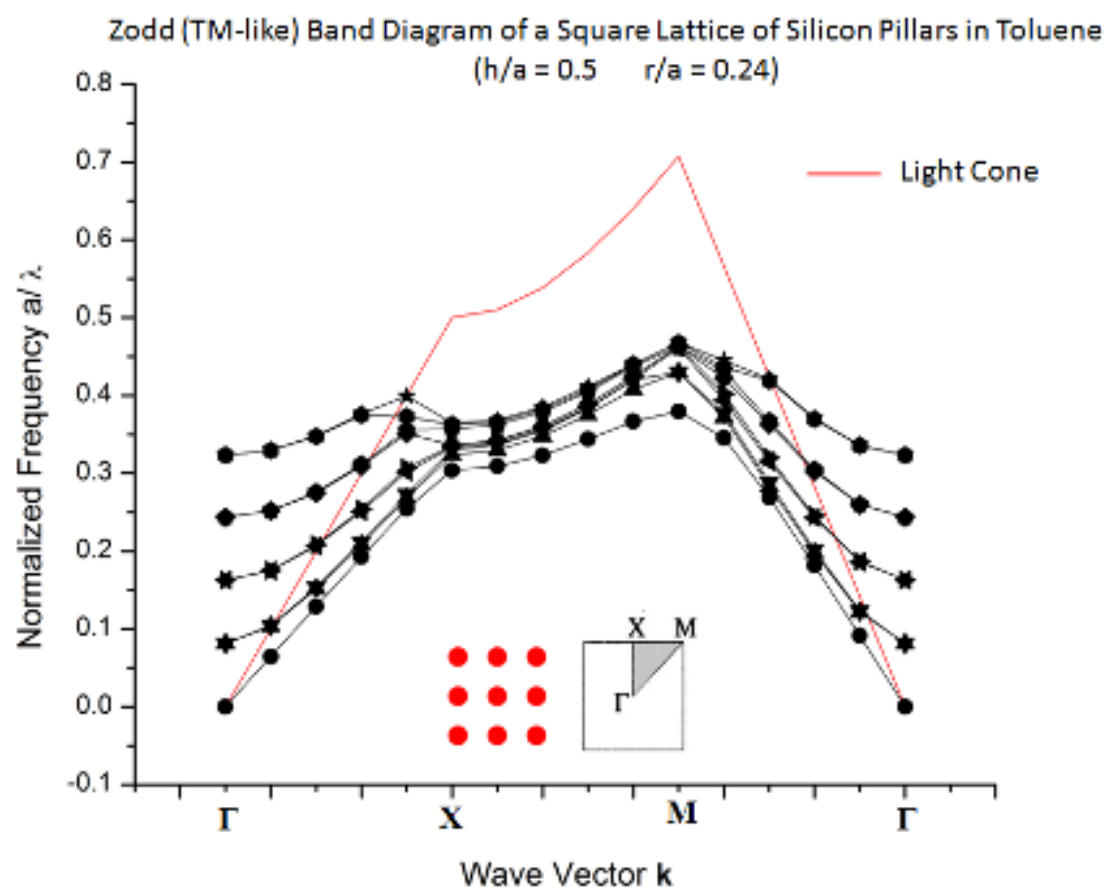

Figure 3.18. Zodd (TM-like) band diagram of a slab photonic crystal $(\mathrm{h}=0.5 a)$ square lattice of silicon pillars in toluene. Neither direction nor a complete bandgap can be observed for the guided modes.

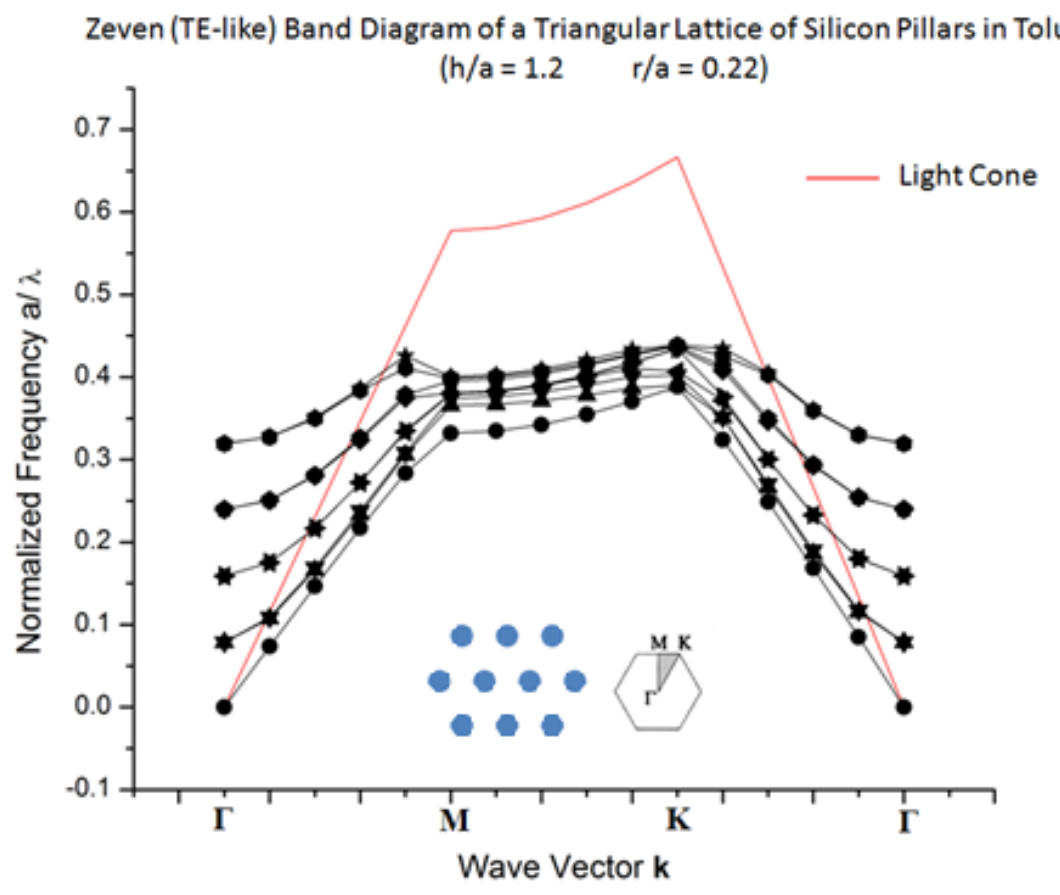

Figure 3.19. Zeven (TE-like) band diagram of a slab photonic crystal triangular lattice $(\mathrm{h}=1.2 a)$ of silicon pillars in toluene. No bandgap can be resolved under the light cone for the guided modes. 


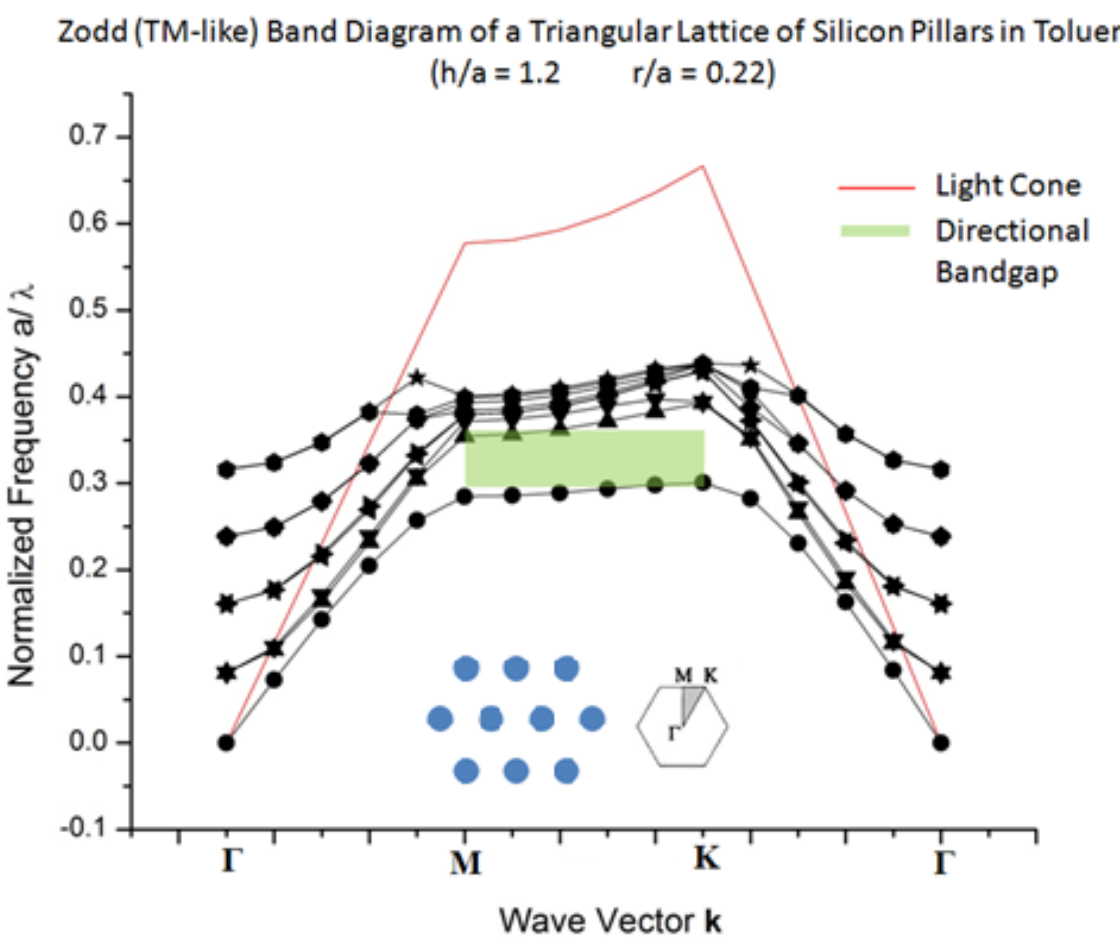

Figure 3.20. Zodd (TM-like) band diagram of a slab photonic crystal triangular lattice $(\mathrm{h}=1.2 a)$ of silicon pillars in toluene. Only a directional bandgap can be resolved in the M to K direction.

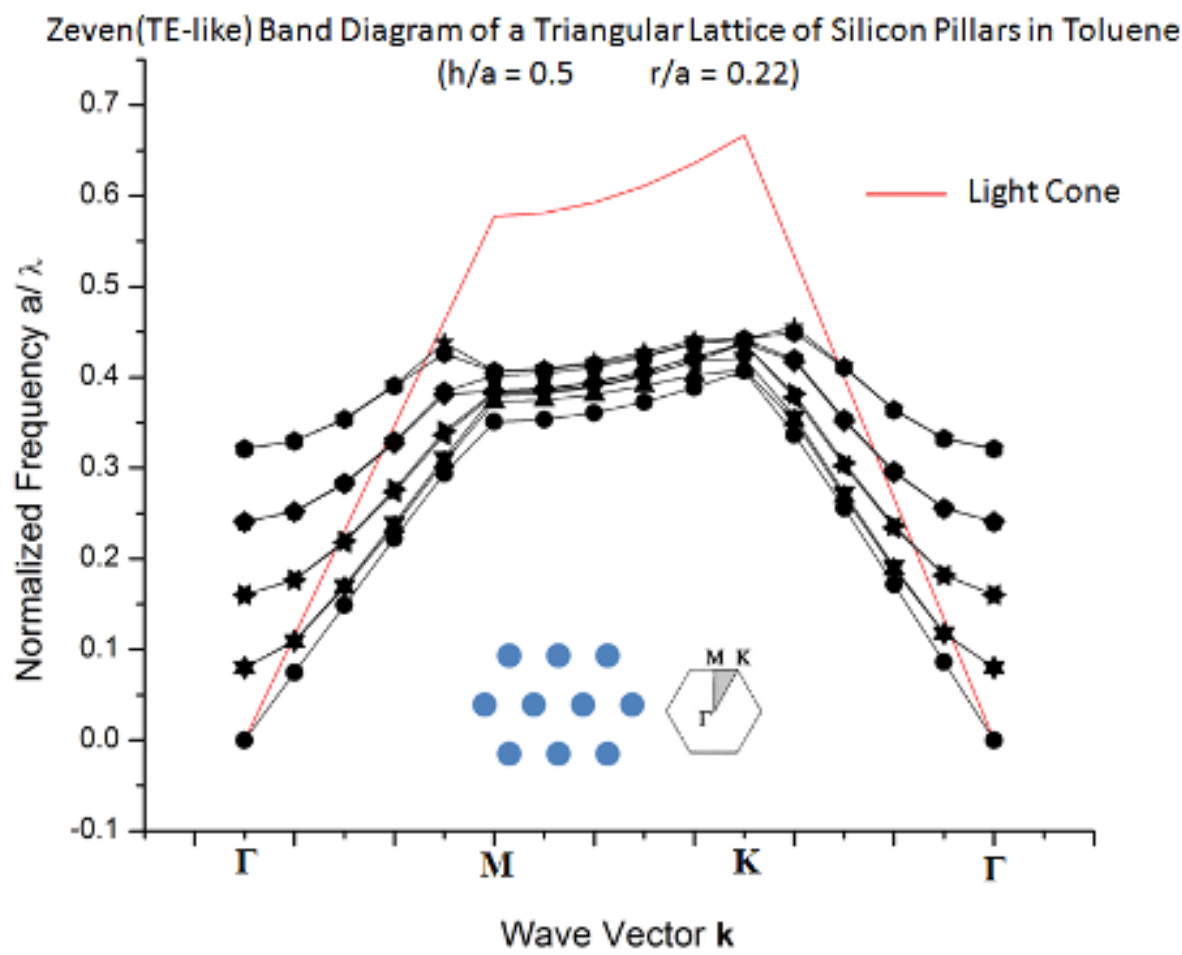

Figure 3.21. Zeven (TE-like) band diagram of a slab photonic crystal triangular lattice $(\mathrm{h}=0.5 a)$ of silicon pillars in toluene. No bandgap can be resolved under the light cone for the guided modes 


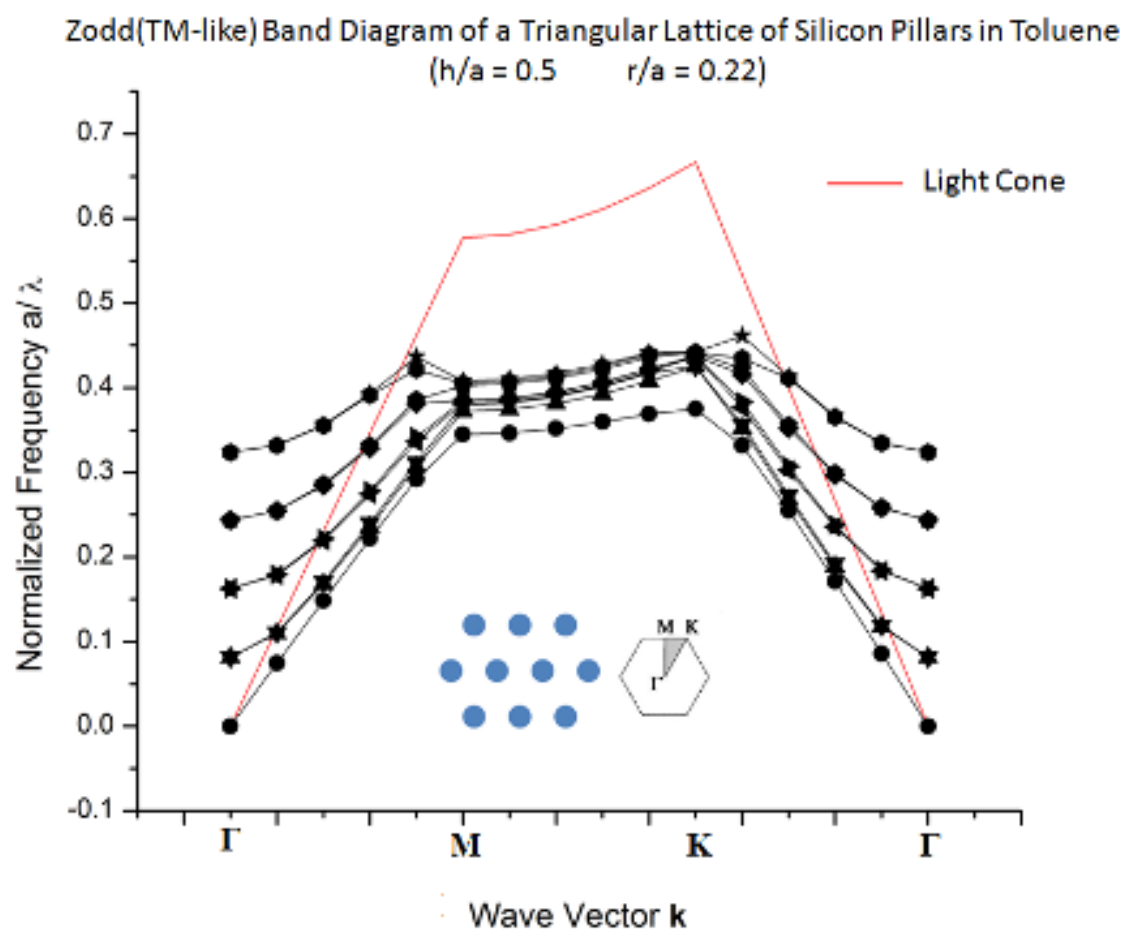

Figure 3.22. Zodd (TM-like) band diagram of a slab photonic crystal triangular lattice $(\mathrm{h}=0.5 a)$ of silicon pillars in toluene. No bandgap can be resolved under the light cone for the guided modes.

Figures 3.23-3.26 demonstrate the band diagrams of the TE- and TM-like polarizations for triangular lattices of toluene holes within silicon and sandwiched in between two $\mathrm{SiO}_{2}$ layers. The band diagrams are shown for those specific lattices, $\mathrm{r} / \mathrm{a}=0.36$, as they demonstrated the widest bandgaps in their infinitely-thick representation. Those diagrams are shown for a slab of the maximum considered thickness, $1.2 a$, and again for a slab with the minimum considered thickness, $0.5 \mathrm{a}$. 


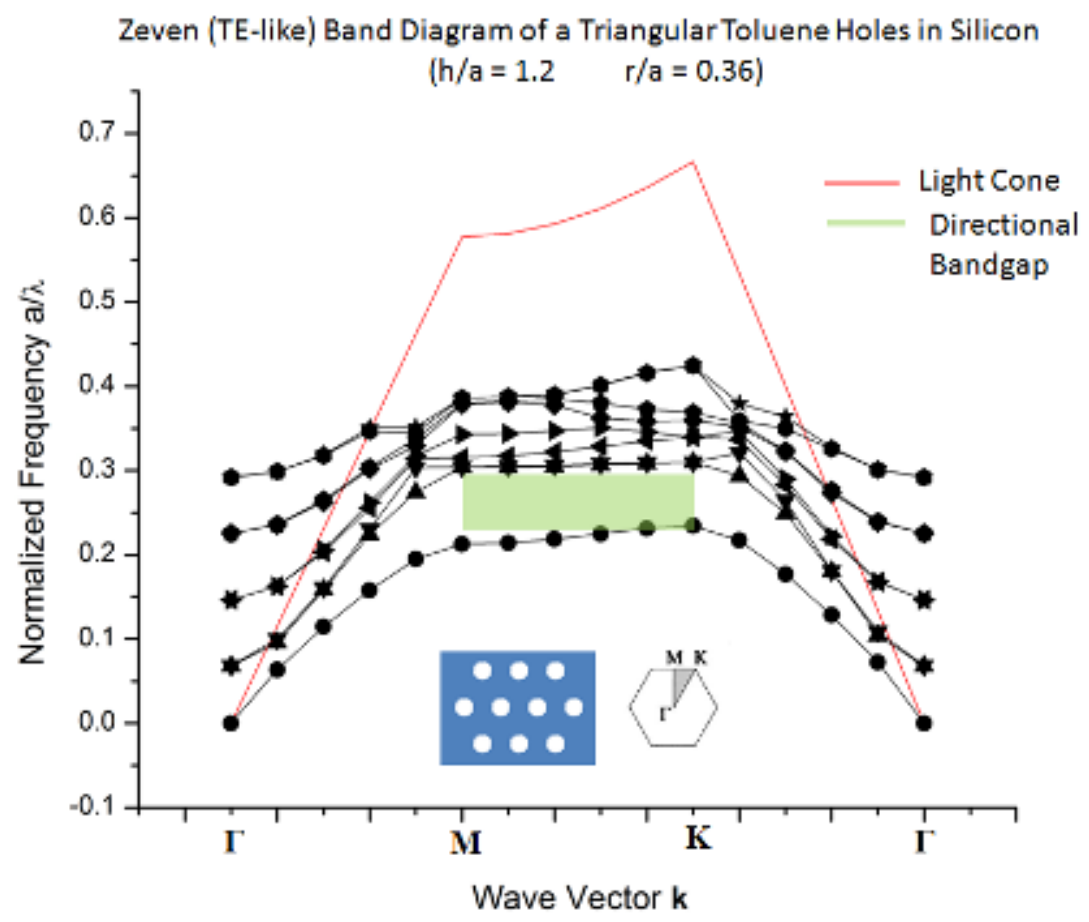

Figure 3.23. Zeven (TE-like) band diagram of a slab photonic crystal triangular lattice $(\mathrm{h}=1.2 a)$ of toluene holes in silicon. Only a directional bandgap between $\mathrm{M}$ and $\mathrm{K}$ can be resolved for the guided modes.

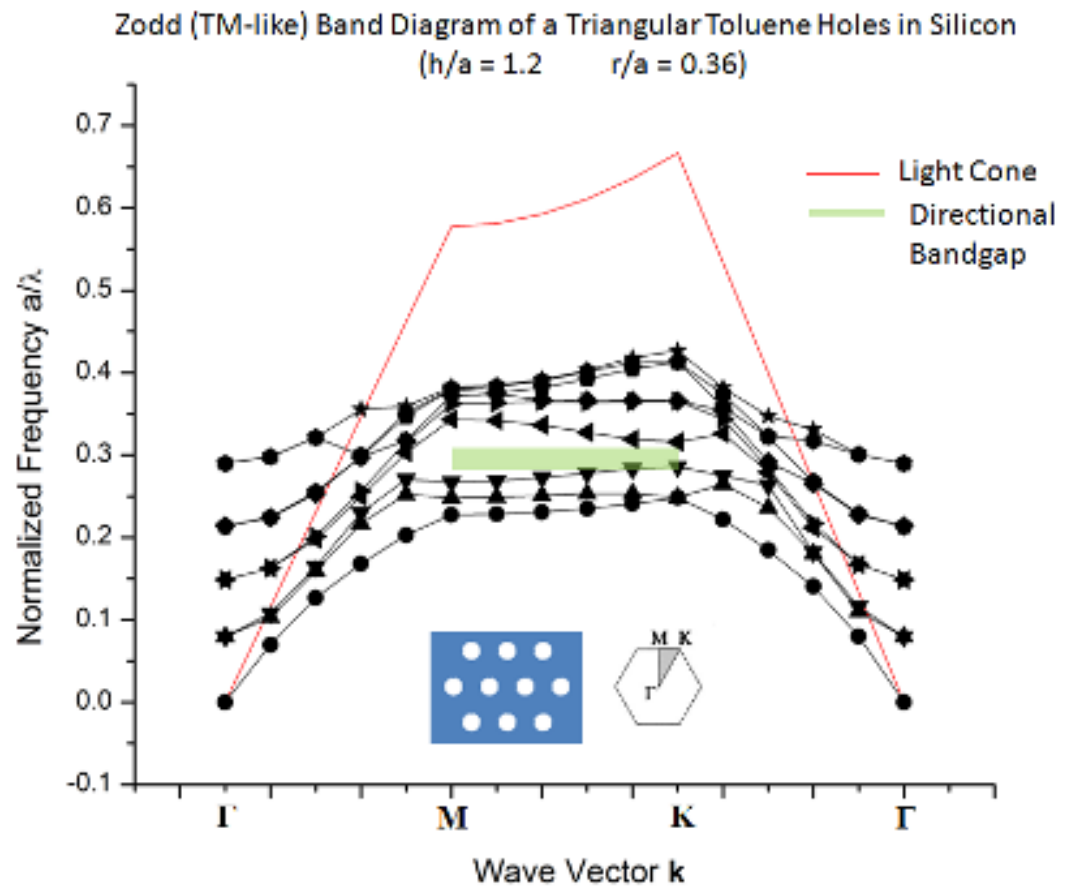

Figure 3.24. Zodd (TM-like) band diagram of a slab photonic crystal triangular lattice $(\mathrm{h}=1.2 a)$ of toluene holes in silicon. Only a very narrow directional bandgap between $\mathrm{M}$ and $\mathrm{K}$ can be resolved for the guided modes. 


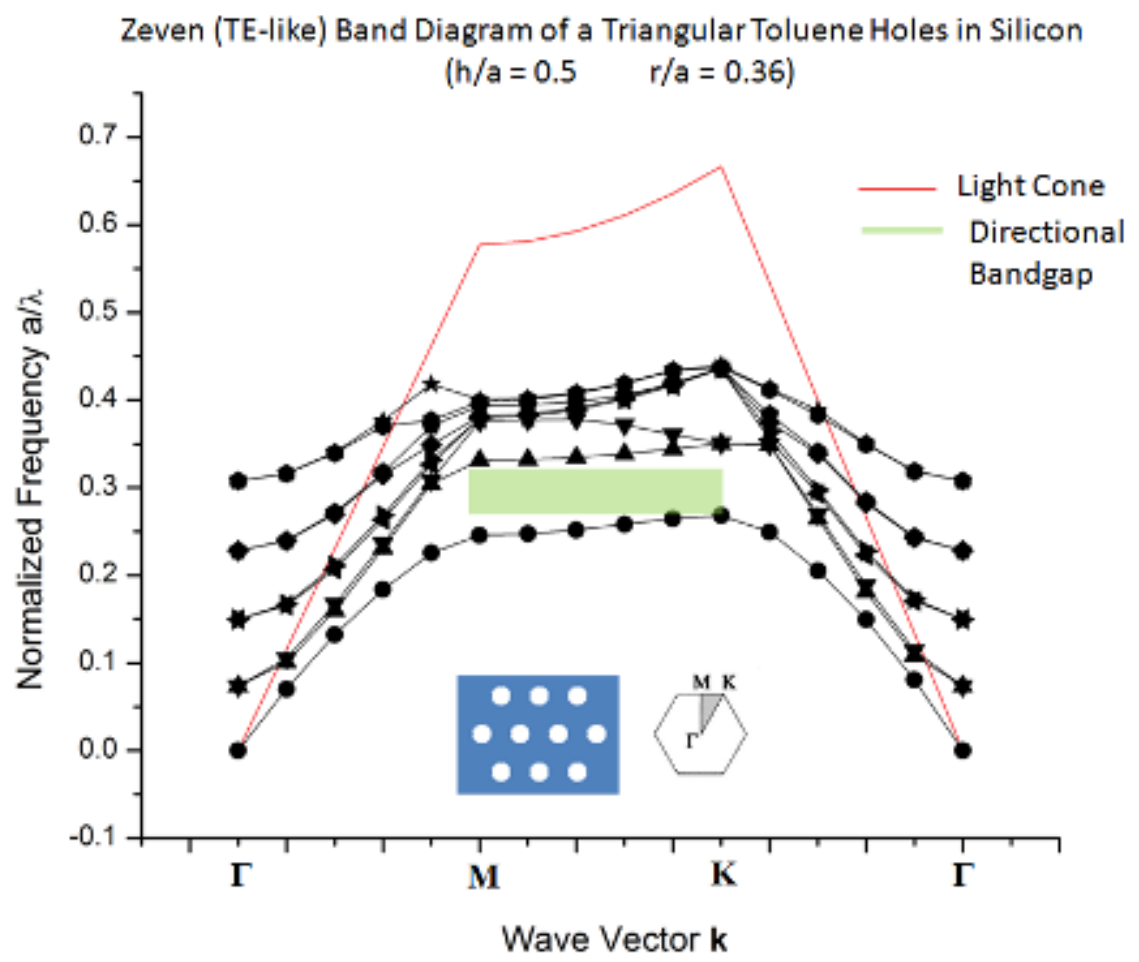

Figure 3.25. Zeven (TE-like) band diagram of a slab photonic crystal triangular lattice $(\mathrm{h}=0.5 a)$ of toluene holes in silicon. Only a directional bandgap between $\mathrm{M}$ and $\mathrm{K}$ can be resolved for the guided modes.

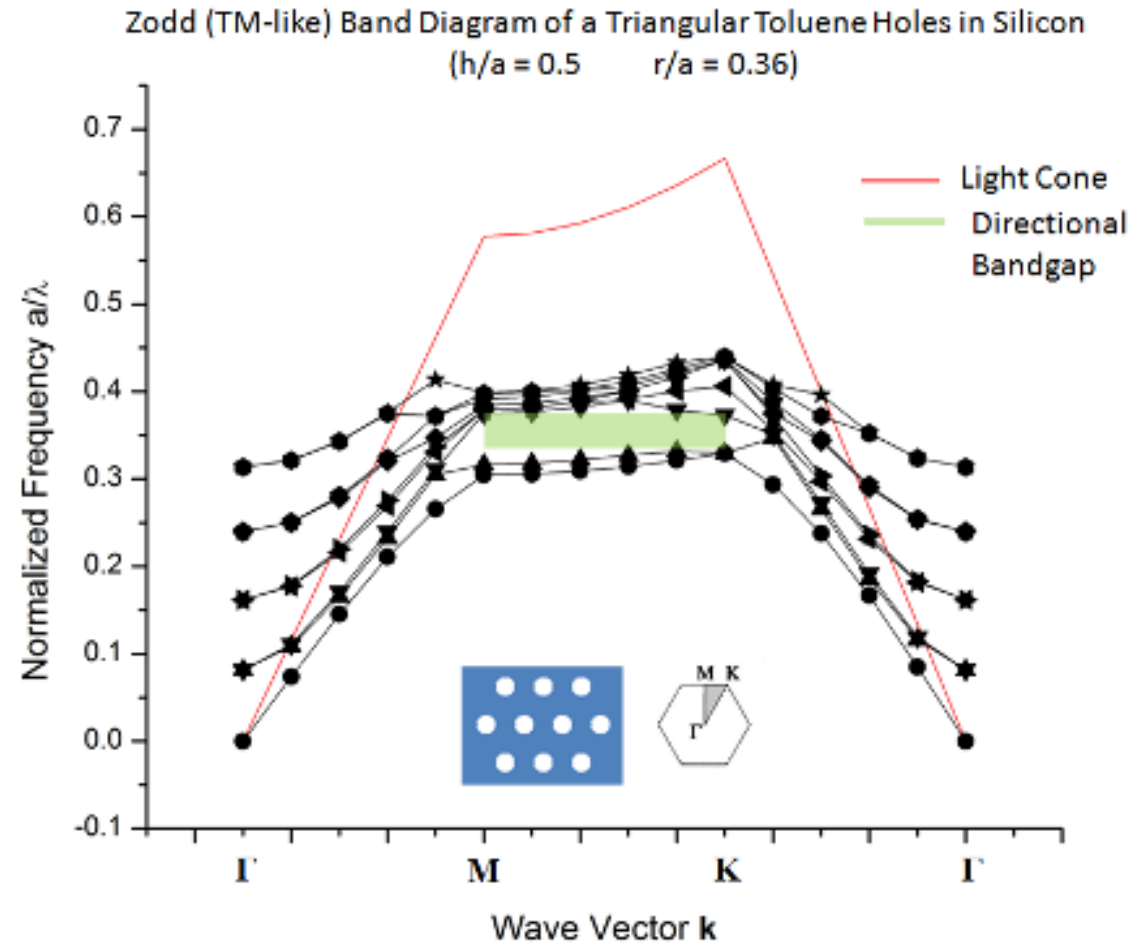

Figure 3.26. Zodd (TM-like) band diagram of a slab photonic crystal triangular lattice $(\mathrm{h}=0.5 a)$ of toluene holes in silicon. Only a directional bandgap between $\mathrm{M}$ and $\mathrm{K}$ can be resolved for the guided modes. 
Due to the lack of a complete photonic bandgap in the previous set of triangular photonic crystal lattices, these lattices were modeled again but this time with an air background below and above the slab. This, as shown in Figures 3.26-3.28, produced complete bandgaps for the guided modes as bands started to lie outside of the light cone near the edges of the irreducible Brillouin zone. Figure 3.27 shows a bandgap map for the range of slab thicknesses between $0.5 a$ and 1.2a. Figures 3.28 and 3.29 demonstrate the TE and TM-like band diagrams for a slab thickness of $0.8 \mathrm{a}$ with a reasonable $13.48 \%$ bandgap for the TE-like modes and $4.69 \%$ for the TM-like modes. The actual extracted data for these lattices are demonstrated in tables 3.8 and 3.9.

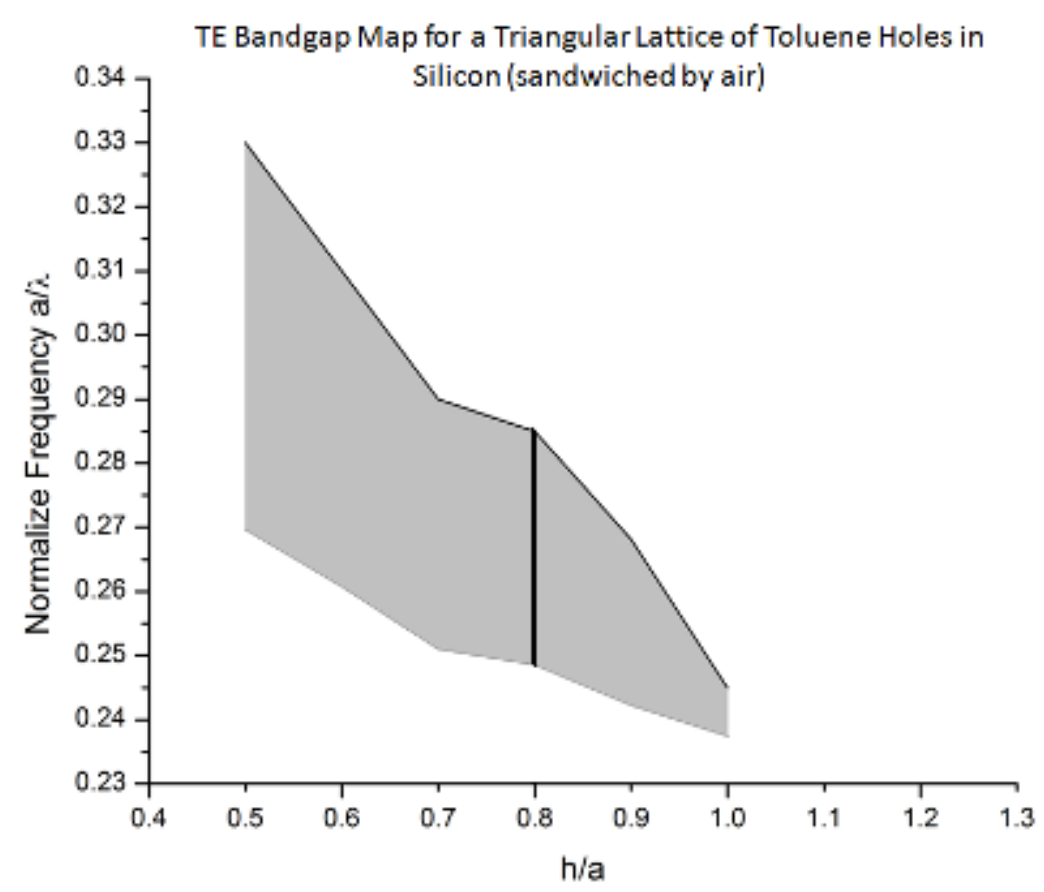

Figure 3.27. TE bandgap for a triangular lattice of slab photonic crystal of toluene holes in silicon sandwiched by air background 


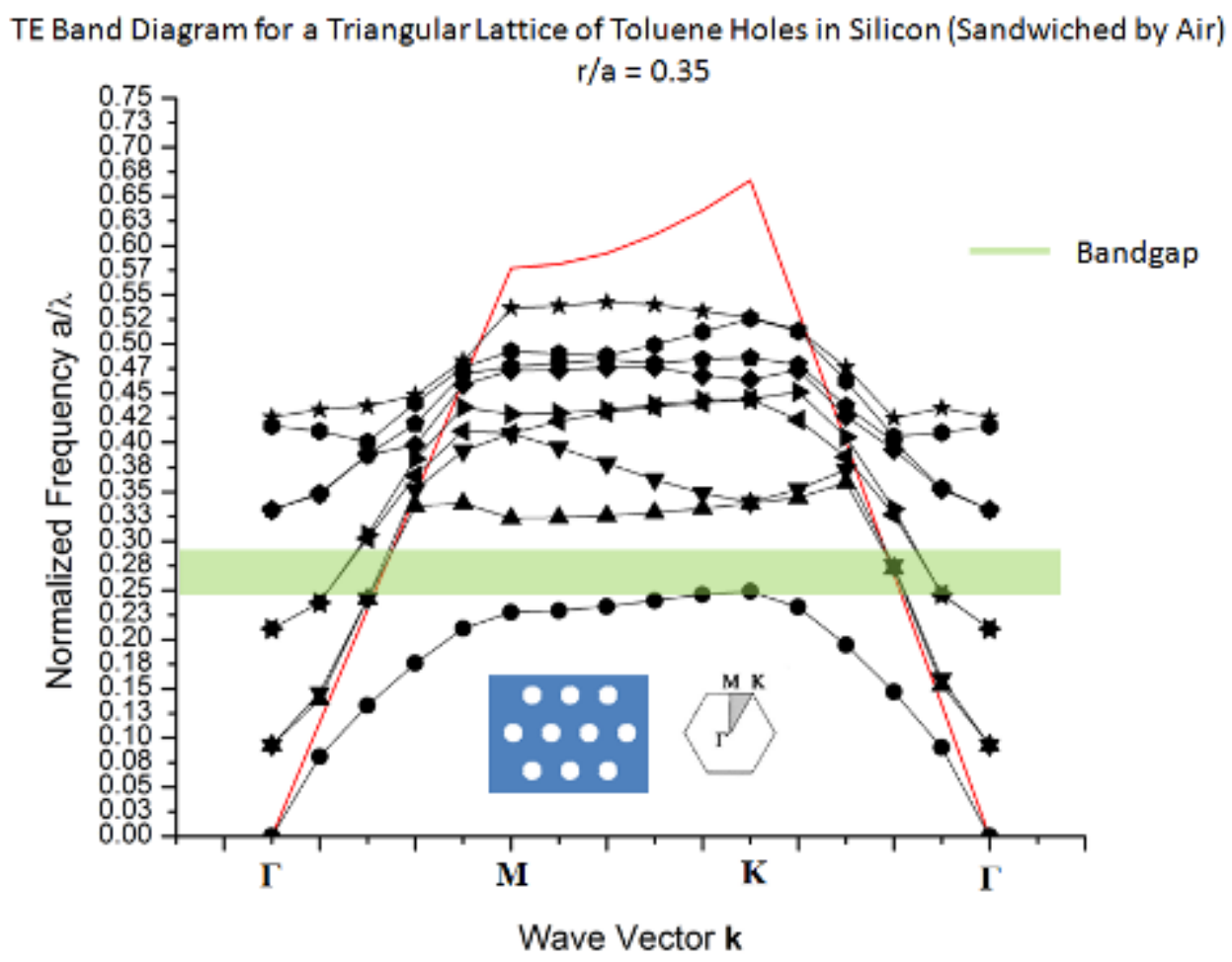

Figure 3.28. TE band diagram for a triangular slab photonic crystal lattice of toluene holes in silicon sandwiched by air. A bandgap of size $13.4 \%$ can be observed.

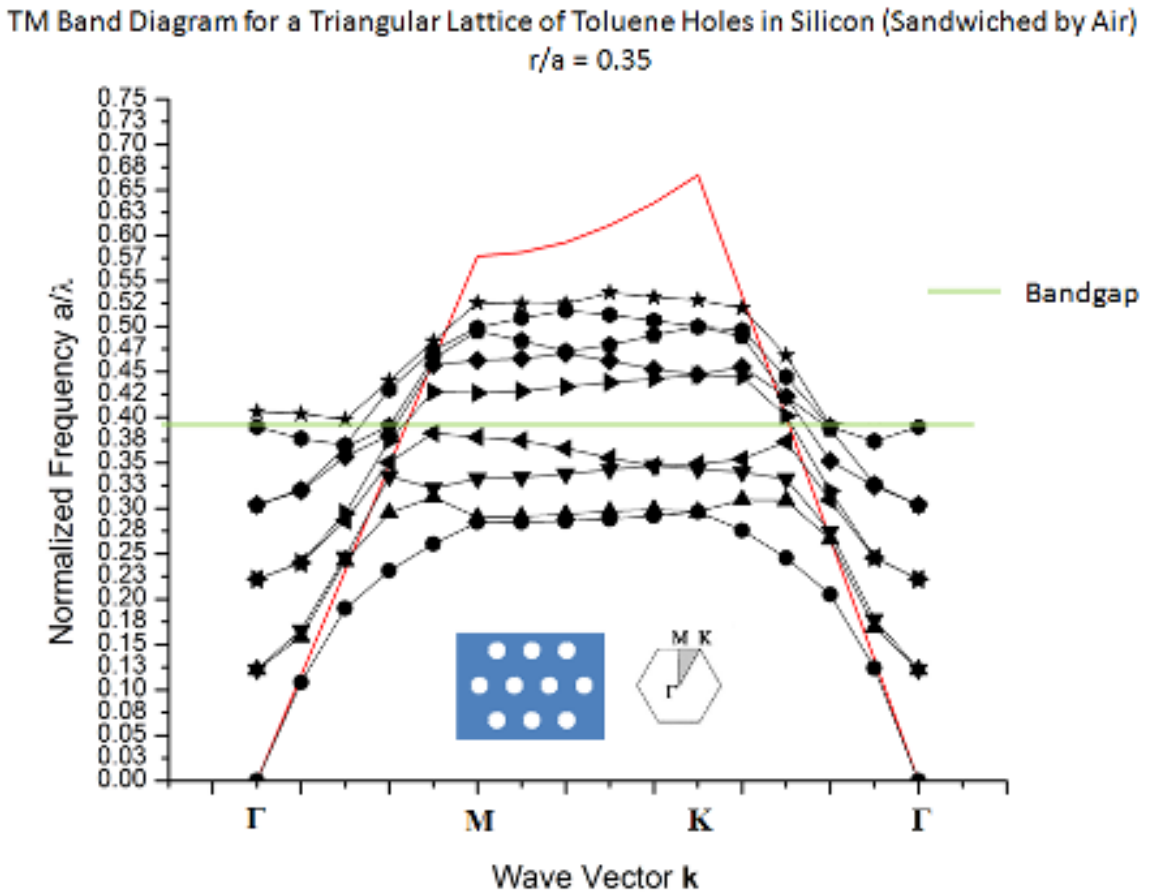

Figure 3.29. TM band diagram for a triangular slab photonic crystal lattice of toluene holes in silicon sandwiched by air. A bandgap of size $4.8 \%$ can be observed between the $4^{\text {th }}$ and $5^{\text {th }}$ bands 
Table 3.8. Table summarizing the bandgap measurement results for TE-like modes of a slab triangular lattice of toluene holes in silicon (sandwiched by air). $\mathrm{r} / \mathrm{a}=0.35 \mathrm{a}$

\begin{tabular}{|c|c|c|c|c|c|}
\hline Height & \multicolumn{5}{|c|}{ Zeven (TE-like) } \\
\hline & Bands & $\begin{array}{c}\text { Gap Bottom Frequency } \\
(\mathrm{a} / \boldsymbol{\lambda})\end{array}$ & Gap Top Frequency $(\mathrm{a} / \boldsymbol{\lambda})$ & Width(\%) & Mid-Gap Frequency $(\mathrm{a} / \boldsymbol{\lambda})$ \\
\hline 0.5 & 1 and2 & 0.269 & 0.33 & 20.3672788 & 0.2995 \\
\hline 0.6 & 1 and 2 & 0.26 & 0.31 & 17.54385965 & 0.285 \\
\hline 0.7 & 1 and 2 & 0.251 & 0.29 & 14.41774492 & 0.2705 \\
\hline 0.8 & 1 and2 & 0.249 & 0.285 & 13.48314607 & 0.267 \\
\hline 0.9 & 1 and2 & 0.242 & 0.2685 & 10.38197845 & 0.25525 \\
\hline 1 & 1 and2 & 0.238 & 0.245 & 2.898550725 & 0.2415 \\
\hline 1.2 & N/A & & & & \\
\hline
\end{tabular}

Table 3.9. Table summarizing the bandgap measurement results for TE-like modes of a slab triangular lattice of toluene holes in silicon (sandwiched by air). $\mathrm{r} / \mathrm{a}=0.35$

\begin{tabular}{|c|c|c|c|c|c|}
\hline Height & \multicolumn{5}{|c|}{ Zodd (TM-like) } \\
\hline & Bands & $\begin{array}{l}\text { Gap Bottom Frequency } \\
\qquad(a / \lambda)\end{array}$ & Gap Top Frequency $(a / \lambda)$ & Width(\%) & Mid-Gap Frequency $(a / \lambda)$ \\
\hline $0.5 \mathrm{a}$ & 4 and 5 & 0.438 & 0.46 & 4.899777283 & \begin{tabular}{|c|}
0.449 \\
\end{tabular} \\
\hline $0.6 \mathrm{a}$ & $\mathrm{N} / \mathrm{A}$ & & & & \\
\hline $0.7 \mathrm{a}$ & $\mathrm{N} / \mathrm{A}$ & & & & \\
\hline $0.8 \mathrm{a}$ & 4 and 5 & 0.383 & 0.401401 & 4.691732927 & 0.3922005 \\
\hline $0.9 \mathrm{a}$ & $\mathrm{N} / \mathrm{A}$ & & & & \\
\hline $1 \mathrm{a}$ & 4 and5 & 0.361 & 0.385 & 6.434316354 & 0.373 \\
\hline $1.2 \mathrm{a}$ & $\mathrm{N} / \mathrm{A}$ & & & & \\
\hline
\end{tabular}

\subsubsection{Analyzing Slab Photonic Crystals of Silicon, Toluene, and Air}

Throughout section 3.3.3, modeling results of the slab photonic crystal lattices that demonstrated wide bandgap in their infinitely thick lattices were demonstrated. The band diagram of all lattices sandwiched between toluene or $\mathrm{SiO}_{2}$ lacked a complete photonic bandgap or even complete TE- or TMlike photonic bandgaps for all wave vector $\mathbf{k}$ directions. Only directional photonic bandgaps could be resolved between the $\mathrm{X}$ and $\mathrm{M}$ edges and the $\mathrm{M}$ and $\mathrm{K}$ edges of the irreducible Brillouin zone for the square and triangular lattices, respectively. This is due to the higher refractive index of the background that creates mode solutions within the bandgap which cancels its complete existence. This may indeed be a problem for our biosensor design since it is expected to operate with liquid solutions that have higher refractive index than that of air. However, when carrying out the characterization experiment, as will be discussed in chapter 5, partial directional photonic bandgaps still demonstrated a resonance effect and an emission enhancement when the IR-emitting quantum dots were excited from within the PhC slab.

Complete photonic bandgaps can be observed if the background material above and below the slab is changed to that of air. In Table 3.10, summarized results of the actual dimensions of the lattice with a 
complete TE bandgap can be found. To calculate the actual lattice constant for a lattice with an r/a that is equal to 0.35 , a point within the photonic bandgap, normally the mid-gap, is considered. That point represents a normalized frequency value that is equal to the lattice constant divided by the wavelength to be controlled $(\mathrm{a} / \lambda)$. For example, from Figure 3.28, the mid-gap point is approximately 0.267 . Therefore, the lattice constant of triangular photonic crystal lattice that would prohibit $1100 \mathrm{~nm}$ light from propagating through it is:

$$
\begin{gathered}
\frac{a}{\lambda}=0.267 \\
\frac{a}{1100}=0.267 \\
a=1100 \times 0.267=293.7 \mathrm{~nm}
\end{gathered}
$$

The radius is $0.35 a$. Therefore, it is equal to $102.79 \mathrm{~nm}$ and the thickness $(0.8 \mathrm{a})$ is equal to $234.9 \mathrm{~nm}$.

\begin{tabular}{|c|c|c|c|c|c|c|c|}
\hline \multicolumn{8}{|c|}{$\begin{array}{c}\text { TriangularLattice of Toluene Holes in Silicon (Sandwiched by Air) } \\
\text { Zeven(TE-like), r/a }=0.35\end{array}$} \\
\hline Height $\mid$ Bands & Gap Bottom Frequency $(a / \lambda)$ & Gap Top Frequency $(a / \lambda)$ & Width(\%) & Mid-Gap Frequency $(\mathrm{a} / \mathrm{\lambda})$ & Lattice Canstant(a) [nm] & $\operatorname{radius}(\mathrm{r})[\mathrm{nm}]$ & Height $(\mathrm{h})[\mathrm{nm}]$ \\
\hline \begin{tabular}{|l|l|}
$0.5 \mathrm{a}$ & 1 and 2 \\
\end{tabular} & \begin{tabular}{|c|}
0.269 \\
\end{tabular} & 0.33 & 20.3672788 & 0.2995 & 329.45 & 115.3075 & 164.725 \\
\hline \begin{tabular}{l|l|}
$0.6 \mathrm{a}$ & 1 and 2 \\
\end{tabular} & 0.26 & 0.31 & 17.54385965 & 0.285 & 313.5 & 109.725 & 188.1 \\
\hline \begin{tabular}{l|l|}
$0.7 \mathrm{a}$ & 1 and 2 \\
\end{tabular} & 0.251 & 0.29 & 14.41774492 & 0.2705 & 297.55 & 104.1425 & 208.285 \\
\hline \begin{tabular}{l|l|}
$0.8 \mathrm{a}$ & 1 and 2 \\
\end{tabular} & 0.249 & 0.285 & 13.48314607 & 0.267 & 293.7 & 102.795 & 234.96 \\
\hline \begin{tabular}{l|l|}
$0.9 \mathrm{a}$ & 1 and 2 \\
\end{tabular} & 0.242 & 0.2685 & 10.38197845 & 0.25525 & 280.775 & 98.27125 & 252.6975 \\
\hline \begin{tabular}{|l|l|}
$1 \mathrm{a}$ & 1 and 2 \\
\end{tabular} & 0.238 & 0.245 & 2.898550725 & 0.2415 & 265.65 & 92.9775 & 265.65 \\
\hline \begin{tabular}{l|l|}
$1.2 \mathrm{a}$ & $\mathrm{N} / \mathrm{A}$ \\
\end{tabular} & & & & & & & \\
\hline
\end{tabular}
These results for the different slab thicknesses are summarized in Table 3.10.

Table 3.10. Dimensions of a triangular lattice of toluene holes in silicon and sandwiched by air

\subsubsection{Time Domain Modeling of a Slab Photonic Crystal Lattice}

MIT Electromagnetic Equation Propagation (MEEP) is a free, finite difference time domain (FDTD), Linux-based software package that can be used to model electromagnetic systems [51]. Previously, MPB was used to search for photonic bandgaps for specific finite-thickness photonic crystal slabs. The lattices that demonstrated photonic bandgap effects are simulated using MEEP to perform numerical calculations and measurements that, to some extent, can mimic real-world measurements. In this section, it is used to demonstrate the bandgap effect and the light confinement capability of photonic crystal lattices in high quality factor resonant cavities.

In the previous sections, it has been demonstrated that for any wavelength (frequency) which falls within the photonic bandgap of a specific lattice, the wave vector associated with the photon traveling at that wavelength through the lattice plane will not be allowed to propagate. Instead, after several periods of 
the lattice, that photon is expected to be confined for several emission periods before leaking out of the plane of the photonic crystal. To demonstrate this effect in MEEP, a finite-thickness photonic crystal slab from the previous section is considered. A triangular lattice of toluene holes inside Silicon sandwiched by air background below and above the slab demonstrated a bandgap between the normalized frequencies 0.251 and 0.29 for a slab of height $h=0.7 a$ and radius $r=0.35 a$. The band diagram for this slab is shown in Figure 3.30

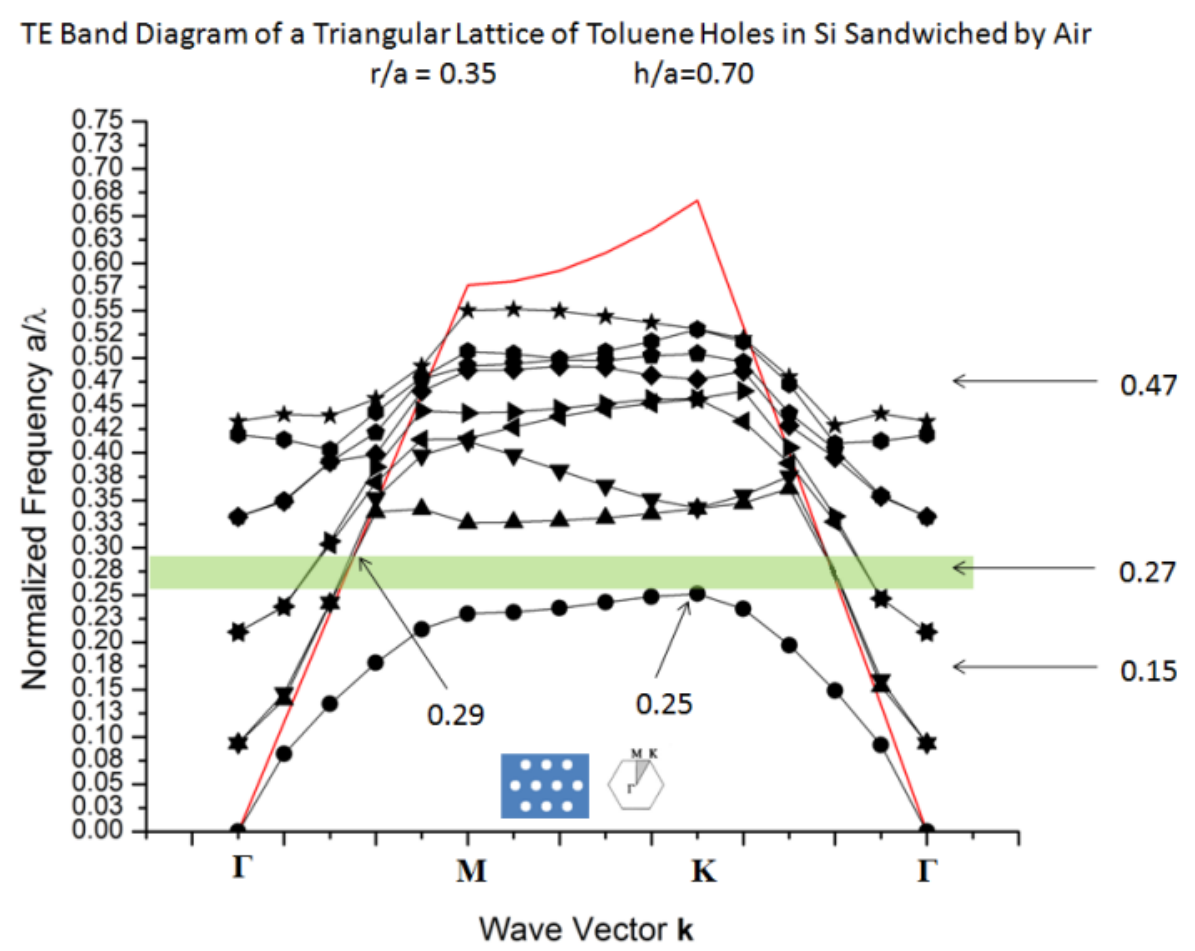

Figure 3.30. TE band diagram of a finite thickness slab of a triangular lattice of toluene holes in Silicon sandwiched by air $(\mathrm{h} / \mathrm{a}=0.7 \mathrm{r} / \mathrm{a}=0.35)$.

In Figure 3.30, the green box indicates the bandgap frequencies for the guided modes under the light cone. To demonstrate the effect of the photonic bandgap on propagating electromagnetic waves, three frequencies are studied: $\sim 0.27,0.15$, and 0.47 . The 0.27 normalized frequency falls inside the bandgap, while the 0.15 and 0.47 normalized frequencies fall below and above the bandgap for this lattice in the dielectric and air bands, respectively. By tailoring the frequency width of a point source emitting a Gaussian spectrum in the central hole of this lattice with 22 periods of holes in the $\mathrm{X}$ and $\mathrm{Y}$ directions (the $\mathrm{PhC}$ plane), the simulated emission can very closely approximate the actual behavior of an emission from a quantum dot inside this exact same hole in an actual fabricated structure. The electric field in the plane of the photonic crystal for each of these three frequencies is demonstrated in Figure 3.31. 
From Figure 3.31, it can be very clearly observed that the electric field intensity for those frequencies that fall outside of the bandgap propagate easily through the photonic crystal plane and leak out of the lattice. In fact, the photonic crystal lattice to waves corresponding to these frequencies will be just like any homogeneous medium that has no effect on its propagation path. On the other hand, when the Gaussian point source in the center of the lattice is designed to emit a frequency that falls within the bandgap (0.278), after $\sim 14$ periods of the photonic crystal lattice in any direction, this emission will no longer be able to propagate and become confined around that source and within the photonic crystal
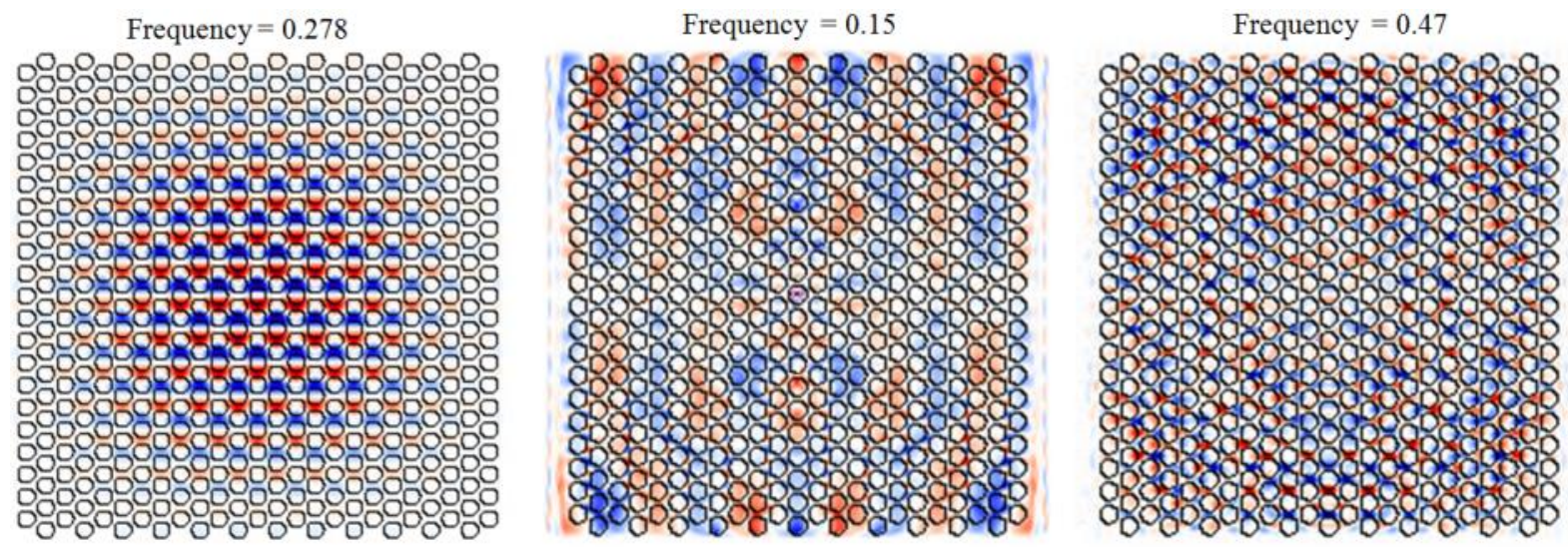

Figure 3.31. Electric field concentration in the plane of the photonic crystal for three frequencies: $0.278, .015$, and 0.47

plane. This effect is called the 'photonic bandgap effect' since the frequency of emission falls within the photonic bandgap.

When altering the periodicity of the photonic crystal by introducing defects in the center surrounding the emission location, the defect mode can be very carefully tuned to create mode solutions within the photonic bandgap. In other words, light can be confined into much smaller designed regions with very high quality factors. The defect discussed here is an L7 defect in which 7 adjacent lattice locations are removed from the central column and one single opening in the center is left but with a smaller r/a ratio that the rest of the lattice. Figure 3.32 demonstrates this defect introduction method. For frequencies that are outside of the photonic bandgap, the defect region has no effect on the propagating wavelength. However, for those frequencies within the bandgap, the defect region will confine light spatially with high quality factors. The quality factor for the defect demonstrated in this Figure is recorded to be around 565. Smaller and higher quality factor defects can be designed. However, for the objective of being able to easily observe the defect region in a regular microscope setting with a low resolution IR camera, larger defects are more desirable.

MEEP can also be used to predict the fluorescence enhancement value of emission within a resonant cavity inside a photonic crystal when compared to an emission without a photonic crystal. A flux plane in 
the vertical direction above the slab is used in the MEEP simulations to collect and sum energy flux until the fields are off and therefore approximating a real-world, time-integrated detector. The flux plane in the simulations is designed with an area that is equal to that of the computational region. In other words, it is completely covering the area above the photonic crystal lattice. Thee flux plane is placed 3 lattice constants above the slab when a photonic crystal lattice is used and 3 lattice constants above the Gaussian point source when there is no photonic crystal. The collected flux around the resonant frequency for both
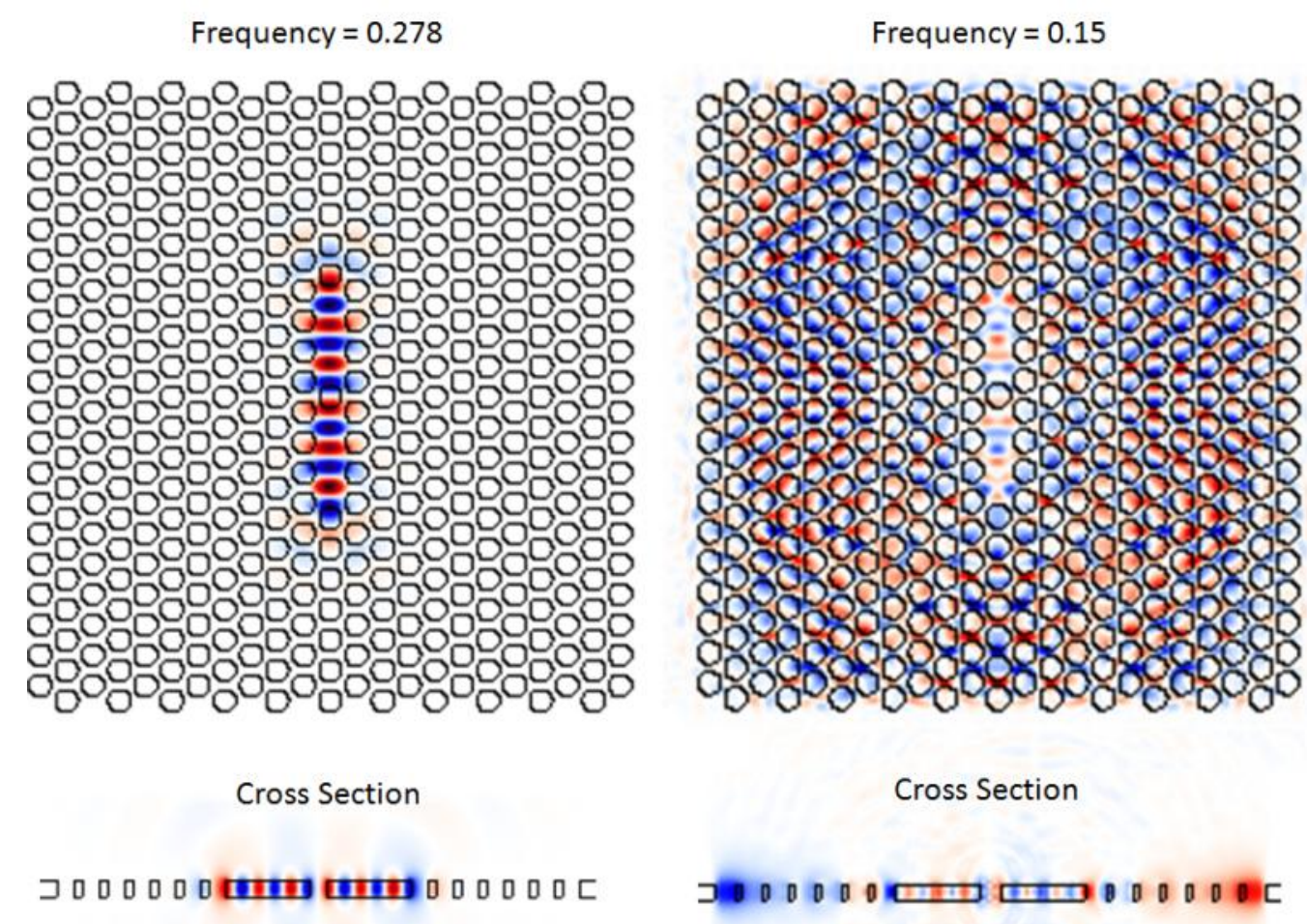

Figure 3.32. Light confinement within a L7 defect for frequencies that fall inside the bandgap only

cases is recorded and approximately 8-fold enhancement can be observed when emission is confined within a resonant cavity and forced to leak out vertically toward the detector. Figure 3.33 summarizes these modeling results. 


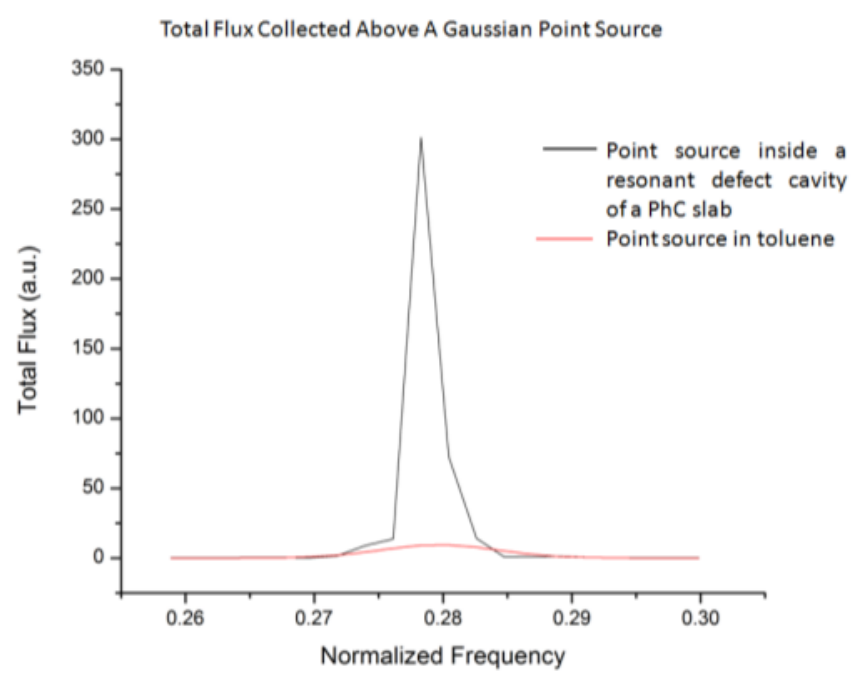

Figure 3.33. Total flux as a function of normalized frequency

\subsection{POLY(DIMETHYLSILOXANE) (PDMS)-BASED PHOTONIC CRYSTALS}

Thin film semiconductor materials are commonly utilized as substrates in the fabrication of $\mathrm{PhCs}$ utilized in sensing platforms due to the large refractive index contrast between these materials and the aqueous solution of the target analytes. This large refractive index contrast makes it easier to design a $\mathrm{PhC}$ that has a complete photonic bandgap. However, one drawback of the use of solid thin films for PhC lattices in biosensors is the increased cost of integrating small pieces of solid materials with polymerbased microfluidic structures. Microfluidic structures in biosensors are primarily fabricated via molding processes in biocompatible materials, which are not readily amenable to the inclusion of sections of semiconductor wafer material containing suspended $\mathrm{PhC}$ films less than 500nm thick. It can be accomplished, but this integration step will significantly increase the final cost of system that utilizes this type of transducer, making them less attractive from a marketing standpoint due to the disposable nature of this type of chem/biosensor component.

$\mathrm{PhC}$ lattices fabricated in polymer materials can provide solutions to the previous limitations. Polymer materials are relatively cheaper than semiconductor materials and can be mass produced using much simpler fabrication processes. However, polymer materials generally have low refractive index which can significantly reduce the desirable effects of the unique light manipulative properties of their $\mathrm{PhC}$ lattices due to the low refractive index contrast between regions of different materials. Therefore, a set of simulations have been carried out to search for a photonic crystal lattice with a complete or partial photonic bandgap in poly(dimethylsiloxane) (PDMS). PDMS is a central component of biocompatible 
microfluidic channels and it has been the material of choice whenever micromolding is carried out. The nanomlding techniques developed to fabricate these lattices in PDMS are discussed in Chapter 4.

The refractive index of PDMS is around 1.43 when mixed and cured with a curing agent at a ratio of 15:1 (PDMS:curing agent). Therefore, frequency-domain modeling using MPB was carried out to reach a conclusion of whether or not PhC lattices composed of this material posses a bandgap. Four lattices were studied:

- Infinitely-thick square photonic crystal lattice of air holes in PDMS

- Infinitely-thick square photonic crystal lattice of PDMS pillars surrounded by air

- Infinitely-thick triangular photonic crystal lattice of air holes in PDMS

- Infinitely-thick triangular photonic crystal lattice of PDMS pillars surrounded by air

MPB modeling of both square lattices of air holes in PDMS and PDMS pillars surrounded by air resulted in no bandgaps for the range of radii between $0.2 a$ to $0.4 a$. This means that light is fully transmitted through these photonic crystal lattices without any attenuation. On the other hand, triangular lattices of air holes in PDMS, as well as PDMS pillars in air, both demonstrated the existence of narrow bandgaps that could be tuned for either surface or resonant-cavity based enhancement. The gap map for each of these lattices, as well as the band diagram for those lattices that demonstrated the widest bandgaps, are shown in Figures 3.34-3.41.

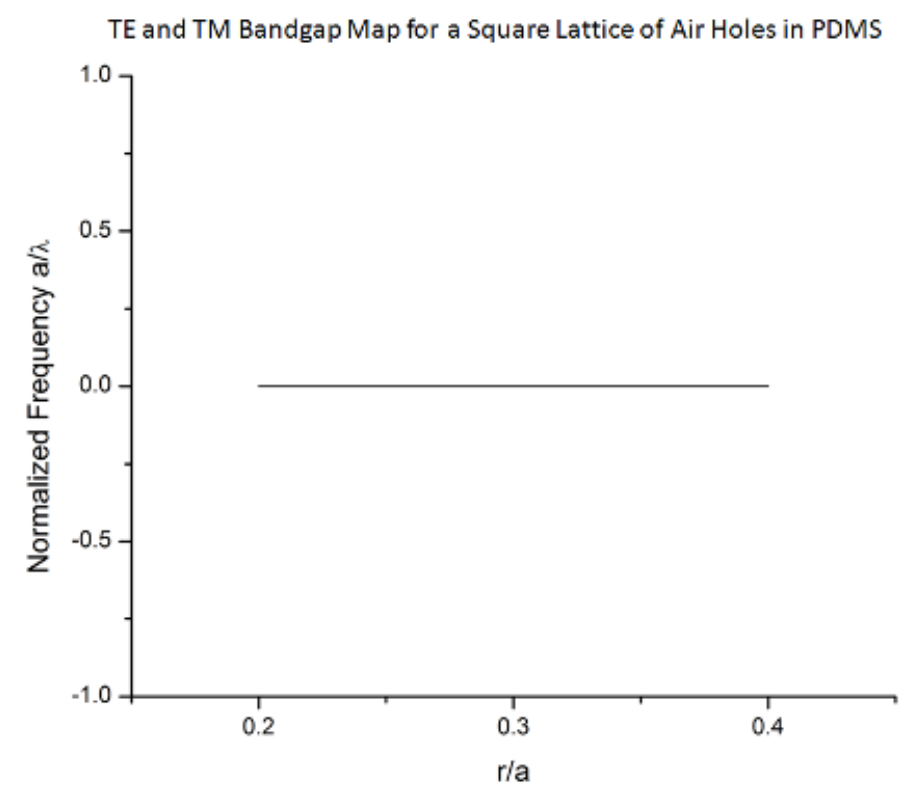

Figure 3.34. TE and TM bandgap map for a square lattice of air holes in PDMS demonstrating no bandgap for radii between $0.2 a$ and $0.4 a$ 


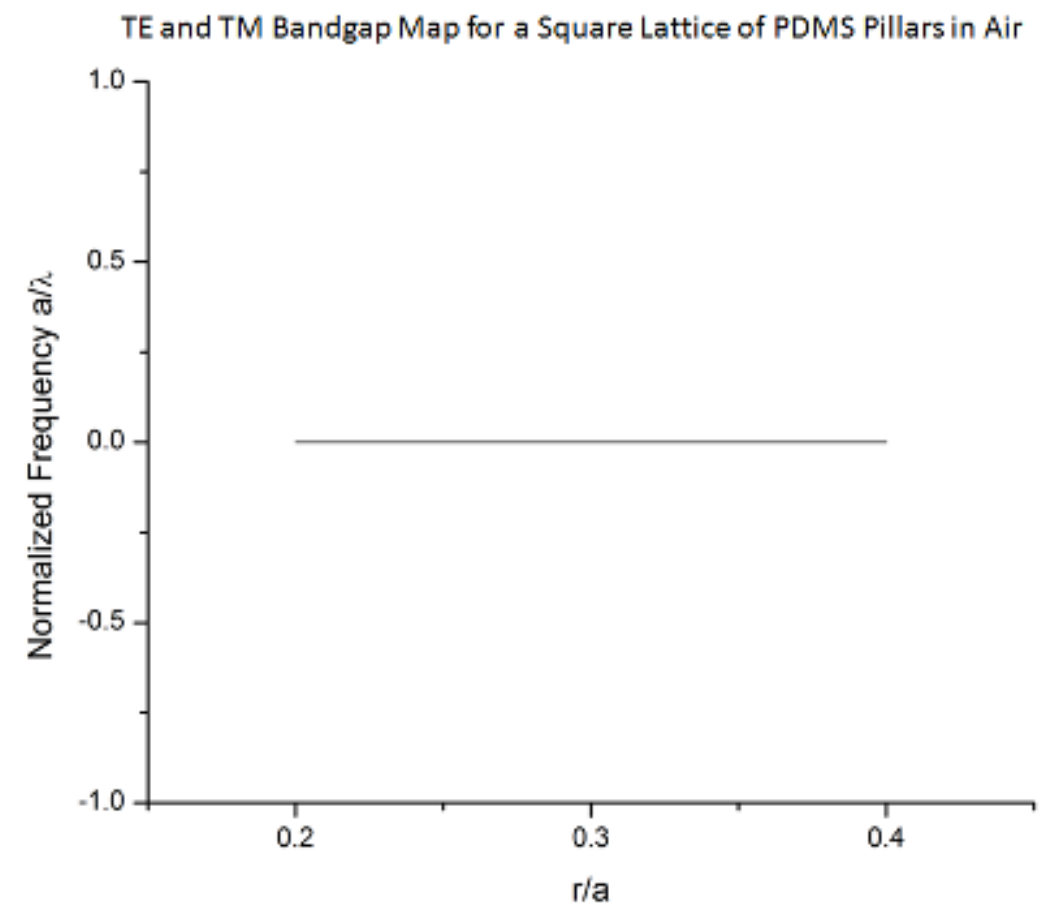

Figure 3.35. TE and TM bandgap map for a square lattice of PDMS pillars in air demonstrating no bandgaps for radii between $0.2 a$ and $0.4 a$

TM Bandgap Map for a Triangular Lattice of Air Holes in PDMS

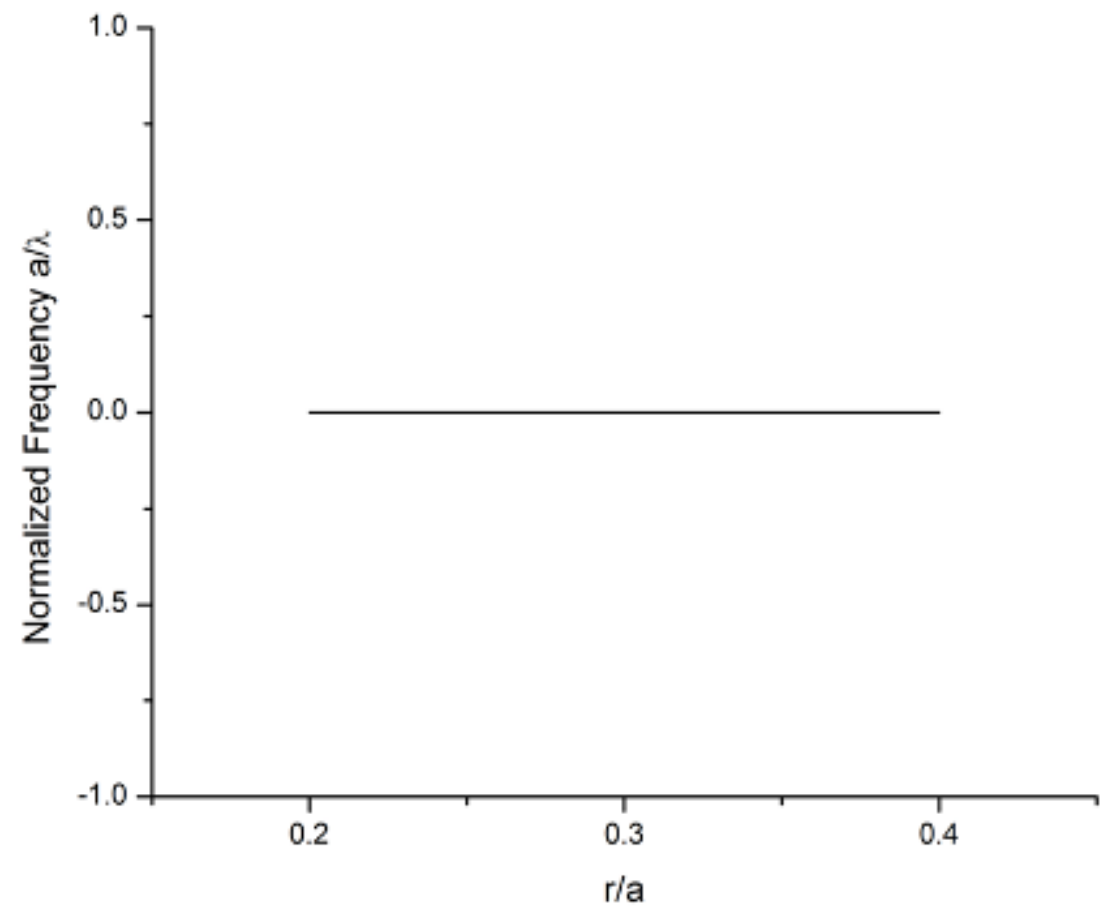

Figure 3.36. TM bandgap map for a triangular lattice of air holes in PDMS demonstrating no bandgaps for radii range between $0.2 a$ and $0.4 a$ 


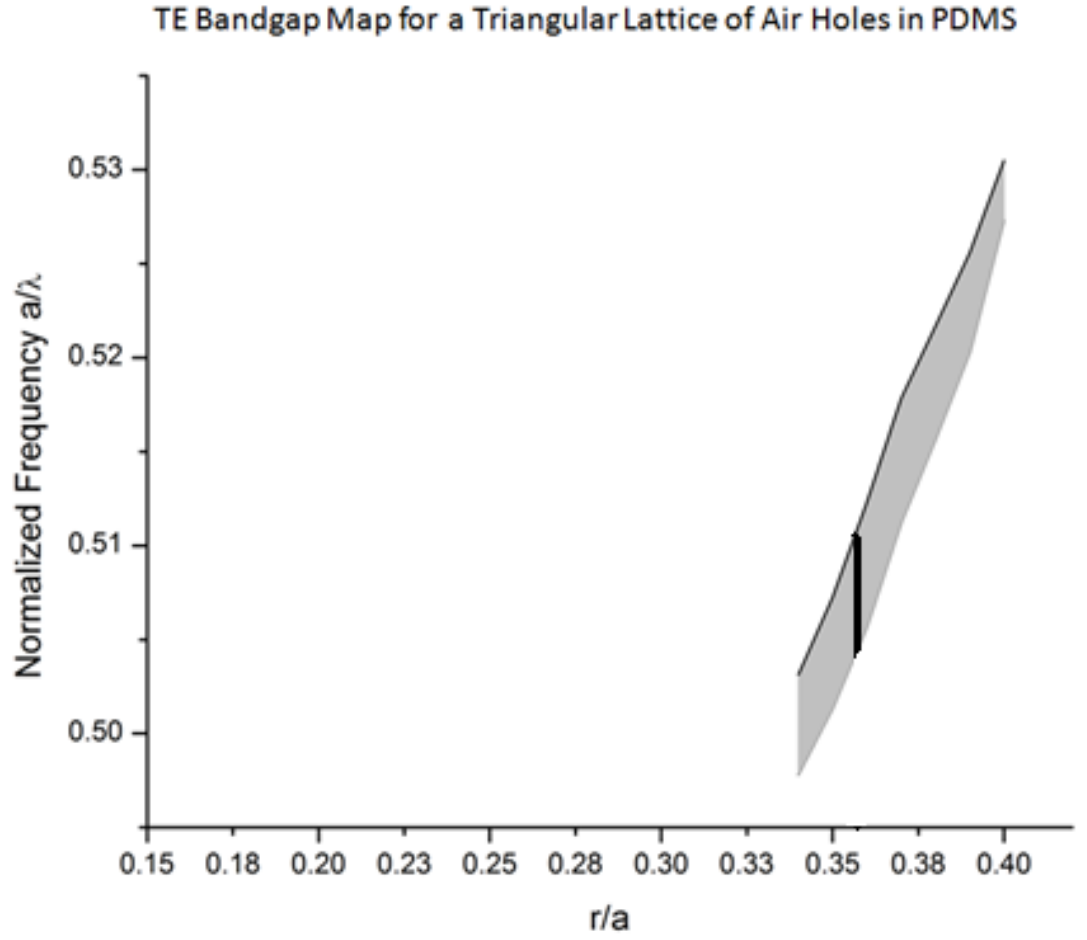

Figure 3.37. TE bandgap map for a triangular lattice of air holes in PDMS

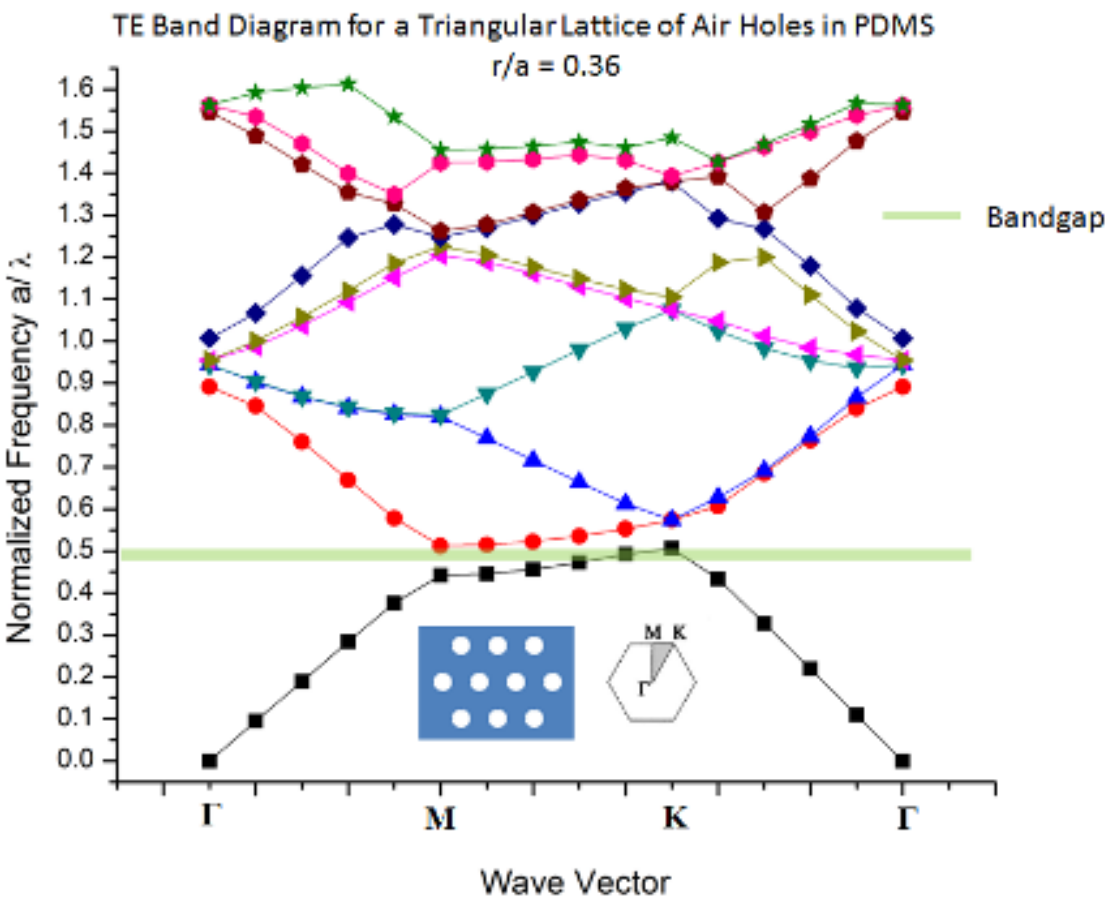

Figure 3.38. TE band diagram of a triangular lattice of air holes in PDMS demonstrating a narrow bandgap $(1.304 \%)$ between the first and second bands for a lattice with $\mathrm{r} / \mathrm{a}=0.36$ 
TM Bandgap Map for a Triangular Lattice of PDMS Pillars in Air

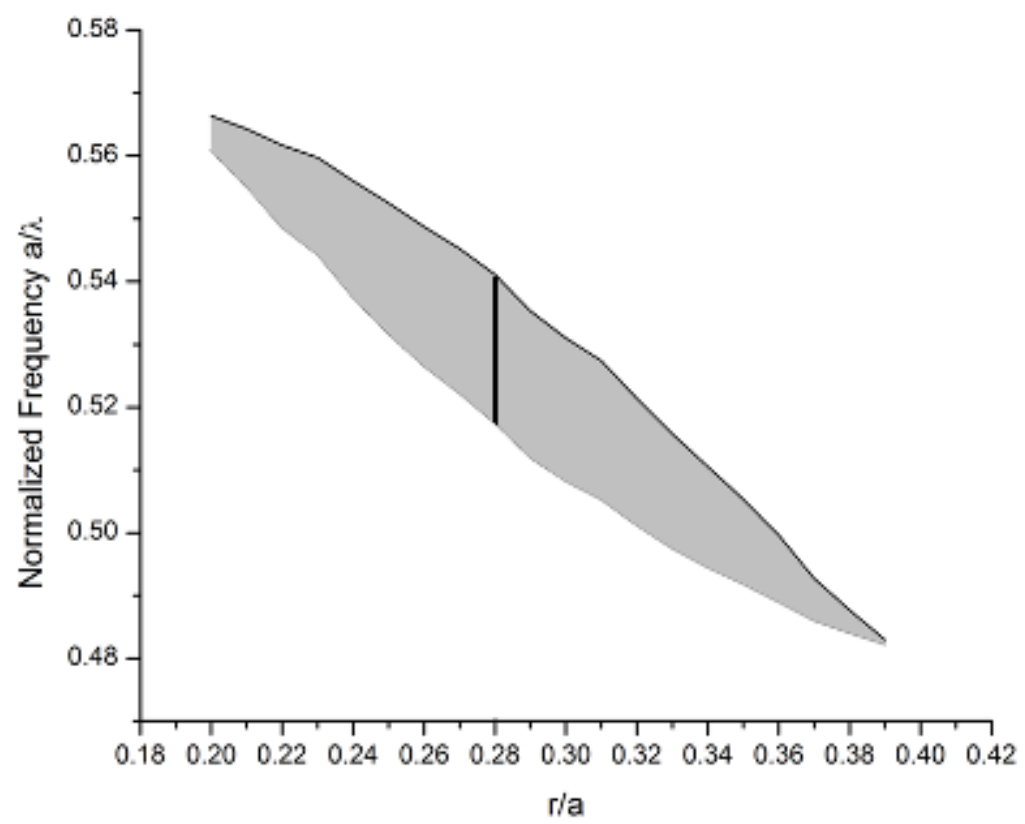

Figure 3.39. Bandgap map of a triangular lattice of PDMS pillars in air. A $4.461 \%$ bandgap at $\mathrm{r} / \mathrm{a}=0.28$ can be observed

TE Bandgap Map for a Triangular Lattice of PDMS Pillars in Air

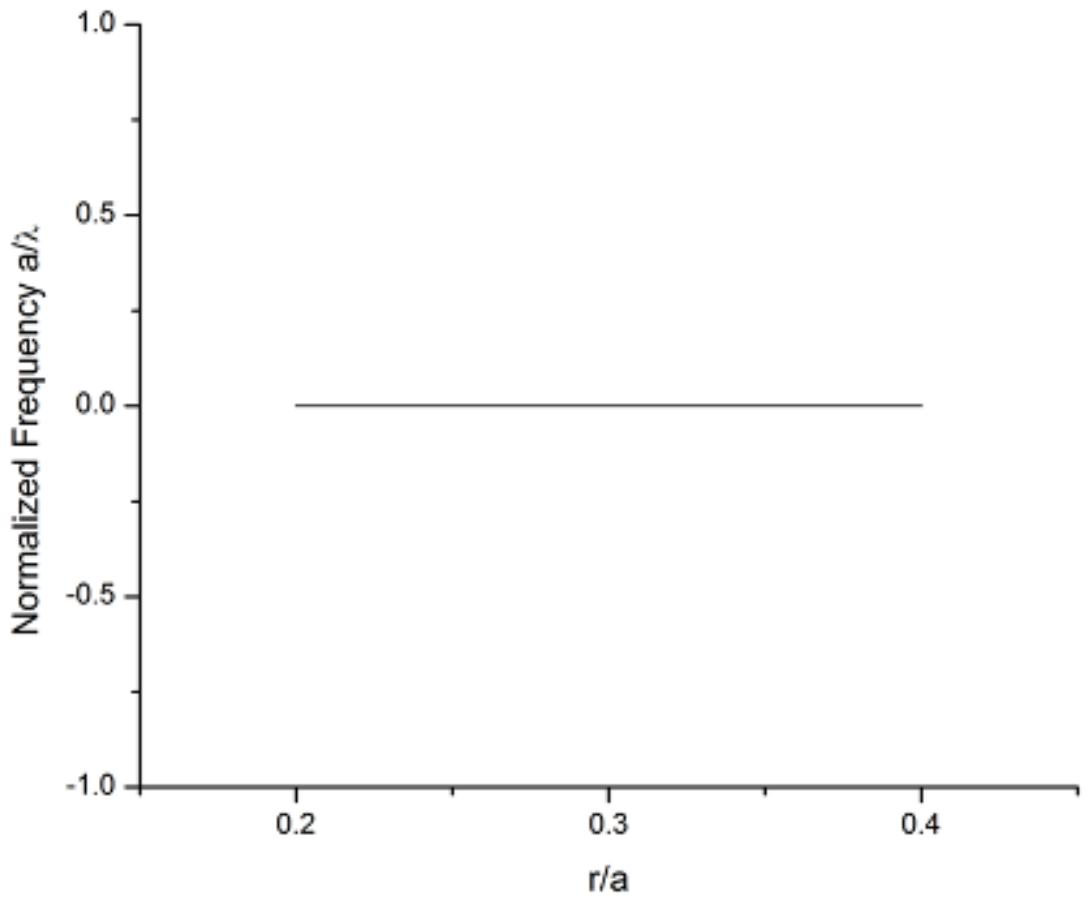

Figure 3.40. TE bandgap map for a triangular lattice PDMS pillars in air. No bandgap is observed 


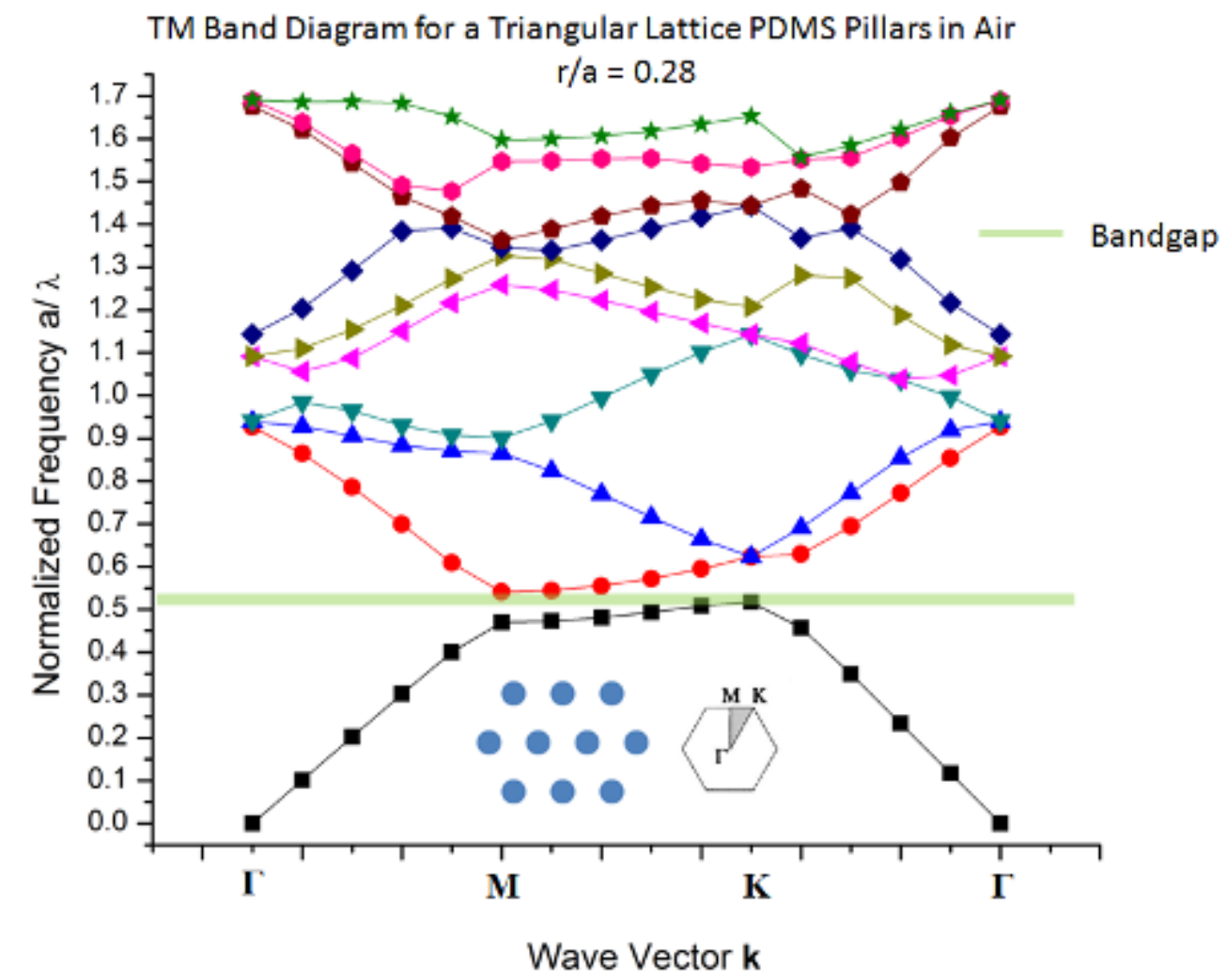

Figure 3.41. TM band diagram of a triangular lattice of PDMS pillars in air demonstrating a bandgap of $4.461 \%$ at $\mathrm{r} / \mathrm{a}=0.28$

\subsubsection{Analyzing Infinitely-Thick Photonic Crystal Lattices of PDMS and Air}

It is very clear from the modeling results that the low refractive index contrast between the high and low dielectric regions of a photonic crystal lattice in PDMS makes it almost impossible to design a lattice with a complete bandgap. However, two lattices demonstrated partial bandgaps. They were:

- Triangular photonic crystal lattice of air holes in PDMS

- Triangular lattice of PDMS pillars surrounded by air

If the low dielectric regions in these previous two lattices replaced by that of any material with a refractive index higher than 1 , bandgaps will no longer exist. The low refractive index contrast in these lattices prevents the separation of the electric or the magnetic field of the propagating electromagnetic wave in the different regions of the lattice, hence no range of prohibited wavelengths will be created. However, for the two previous lattices, it is still desirable to calculate the actual parameters of these 
lattices. Here, the IR-emitting quantum dots are replaced with a visible wavelength emission such as that of fluorescein $(515 \mathrm{~nm})$.

For a triangular lattice of air holes in PDMS, the widest TE bandgap was observed at a lattice with an $r / a$ ratio of 0.36 . Therefore, if the theoretical radius is $0.75 a$, then the actual lattice constant $a$ must be $2.083 a$. This is simply a scaling of the lattice constant so that it can be fabricated while at the same time its dimensions are still in the sub-micron scale to allow control over visible range wavelengths. The actual parameters for this lattice are summarized in Table 3.11.

Table 3.11. Actual lattice parameters of a triangular lattice of air holes in PDMS

\begin{tabular}{|c|c|c|c|c|c|c|c|c|c|}
\hline \multicolumn{10}{|c|}{ PDMS - Triangular Lattice of Air Holes in PDMS } \\
\hline \multicolumn{10}{|c|}{$\mathrm{r} / \mathrm{a}=0.36[$ Scaling: $\mathrm{r}=0.75, \mathrm{a}=2.0833]$} \\
\hline r/a & Bands & $\begin{array}{c}\text { Gap } \\
\text { Bottom } \\
\text { Frequency } \\
(\mathrm{a} / \lambda)\end{array}$ & $\begin{array}{l}\text { Gap Top } \\
\text { Frequency } \\
(\mathrm{a} / \lambda)\end{array}$ & $\begin{array}{l}\text { Mid-gap } \\
\text { Frequency } \\
(\mathrm{a} / \lambda)\end{array}$ & $\begin{array}{c}\text { Gap } \\
\text { Width } \\
(\%)\end{array}$ & $\begin{array}{c}\text { Desired } \\
\text { Wavelength } \\
(\lambda)[\mathrm{nm}]\end{array}$ & $\begin{array}{l}\text { Lattice } \\
\text { Constant } \\
\text { (a) }[\mathrm{nm}]\end{array}$ & $\begin{array}{c}\text { Radius } \\
(\mathrm{r}) \\
{[\mathrm{nm}]}\end{array}$ & $\begin{array}{c}\text { Slab } \\
\text { Thickness } \\
\text { (h) }[\mathrm{nm}]\end{array}$ \\
\hline 0.36 & 1 and 2 & 0.505683 & 0.512 & 0.509 & 1.3039 & 515 & 546.116 & 196.601 & N/A \\
\hline
\end{tabular}

For a triangular lattice of PDMS pillars in air, the widest TM bandgap was observed at a lattice with an $r / a$ ratio of 0.28 . Therefore, if the theoretical radius is $0.75 a$, then the actual lattice constant $a$ must be 2.678a. The actual dimensions for this lattice are summarized in Table 3.12.

Table 3.12. Actual lattice parameters of a triangular lattice of PDMS pillars surrounded by air

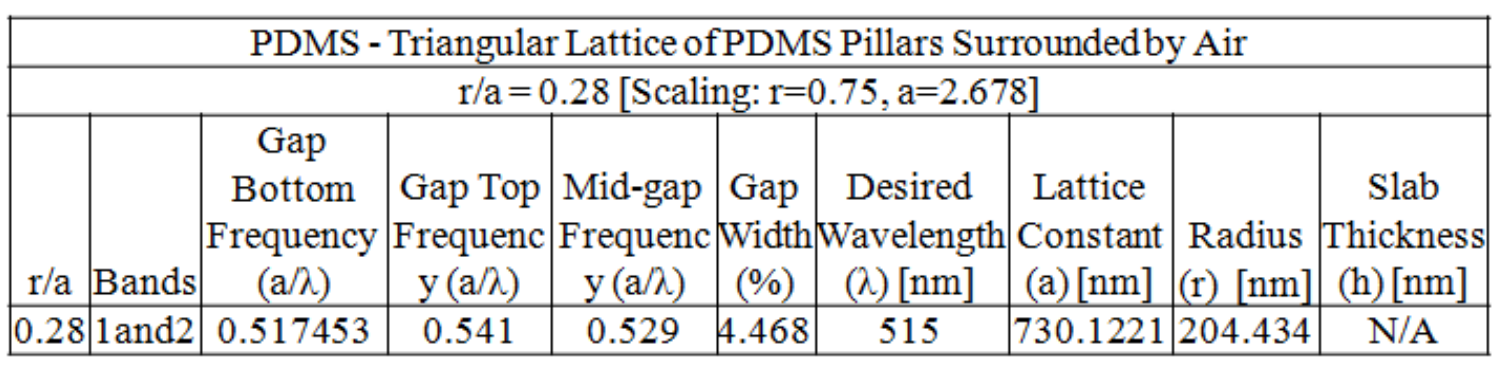




\subsection{EPOXY-BASED PHOTONIC CRYSTALS}

In the previous section, the disadvantages of incorporating thin-film-semiconductor-based PhC lattice as the core structures within their biosensors have been discussed. Another possible polymer material to be considered for molding photonic crystal lattices into is epoxy, Epoxy Technology®. Epoxy's refractive index is approximately 1.5168, which is higher than that of PDMS and can also be made biocompatible. In chapter 4, a novel nanomolding process to produce submicron photonic crystal lattices in epoxy is discussed.

In order to reach a conclusion of whether or not Epoxy is suitable as the substrate material for the $\mathrm{PhC}$ lattices, frequency-domain simulations through MPB were carried out once again. Throughout this section, the bandgap map of 4 different lattices will be demonstrated as well as the band diagrams of the highest bandgaps recorded for each lattice. The four lattices are

- Infinitely-thick square photonic crystal lattice of air holes in Epoxy

- Infinitely-thick square photonic crystal lattice of Epoxy pillars surrounded by air

- Infinitely-thick triangular photonic crystal lattice of air holes in Epoxy

- Infinitely-thick triangular photonic crystal lattice of Epoxy pillars surrounded by air

Once again, square lattices of air holes in Epoxy and Epoxy pillars surrounded by air both demonstrated no bandgaps for the range of radii between $0.2 a$ to $0.4 a$. This means that light is fully transmitted through these photonic crystal lattices without any attenuation. On the other hand, triangular lattices of air holes in Epoxy as well as Epoxy pillars in air both demonstrated the existence of bandgaps that could be tuned for either surface or resonant-cavity based enhancement. The gap map for each of these lattices as well the band diagram that demonstrated the widest bandgaps are shown in Figures 3.423.49 . 
TM and TE Bandgap Map for a Square Lattice of Air Holes in Epoxy

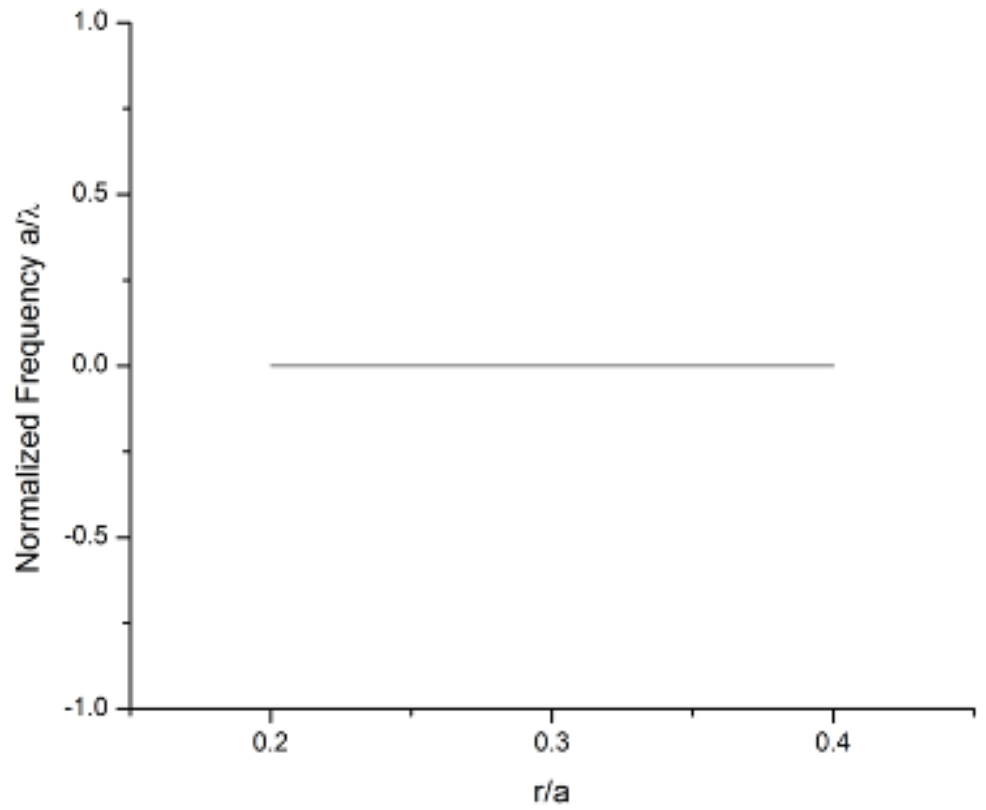

Figure 3.42. TM and TE bandgap map for a square lattice of Air Holes in Epoxy demonstrating no bandgaps of any size for the radii range between $0.2 a$ and $0.4 a$

TM and TM Bandgap Map for a Square Lattice of Epoxy Pillars in Air

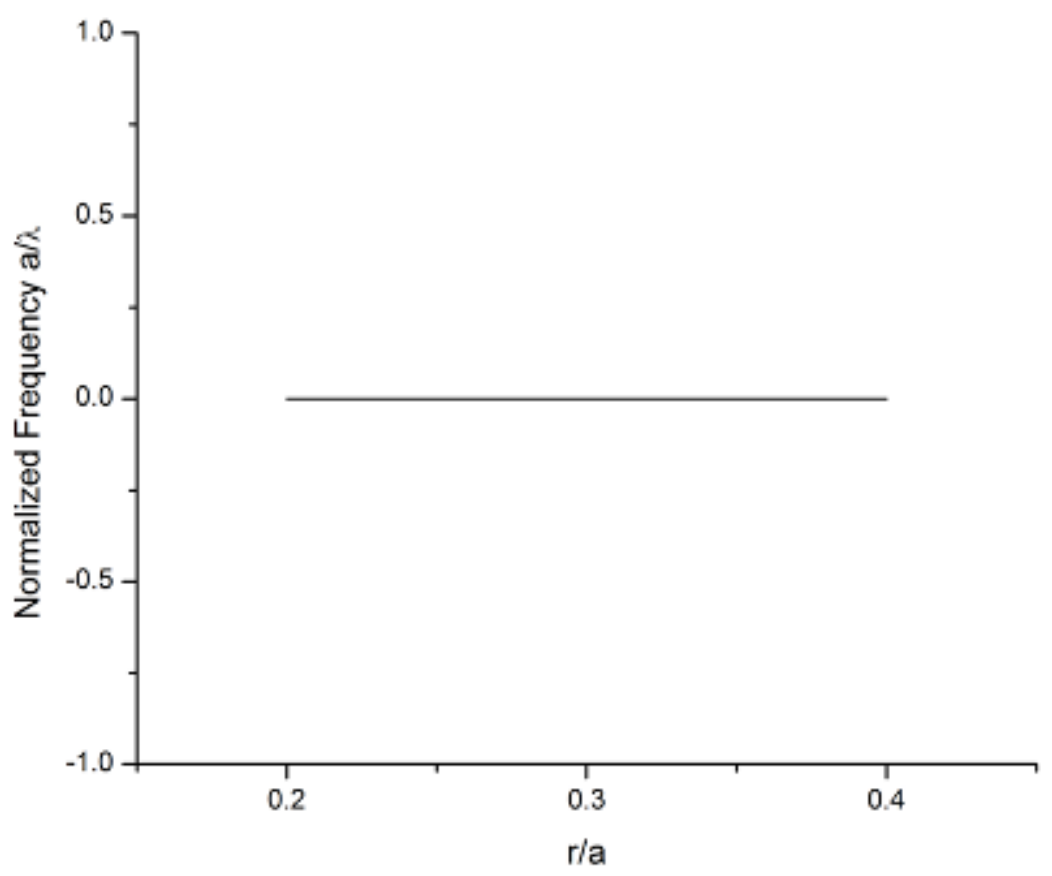

Figure 3.43. TM and TE bandgap map for a square lattice of Epoxy pillars in air demonstrating no bandgaps of any size for the radii range between $0.2 a$ and $0.4 a$ 


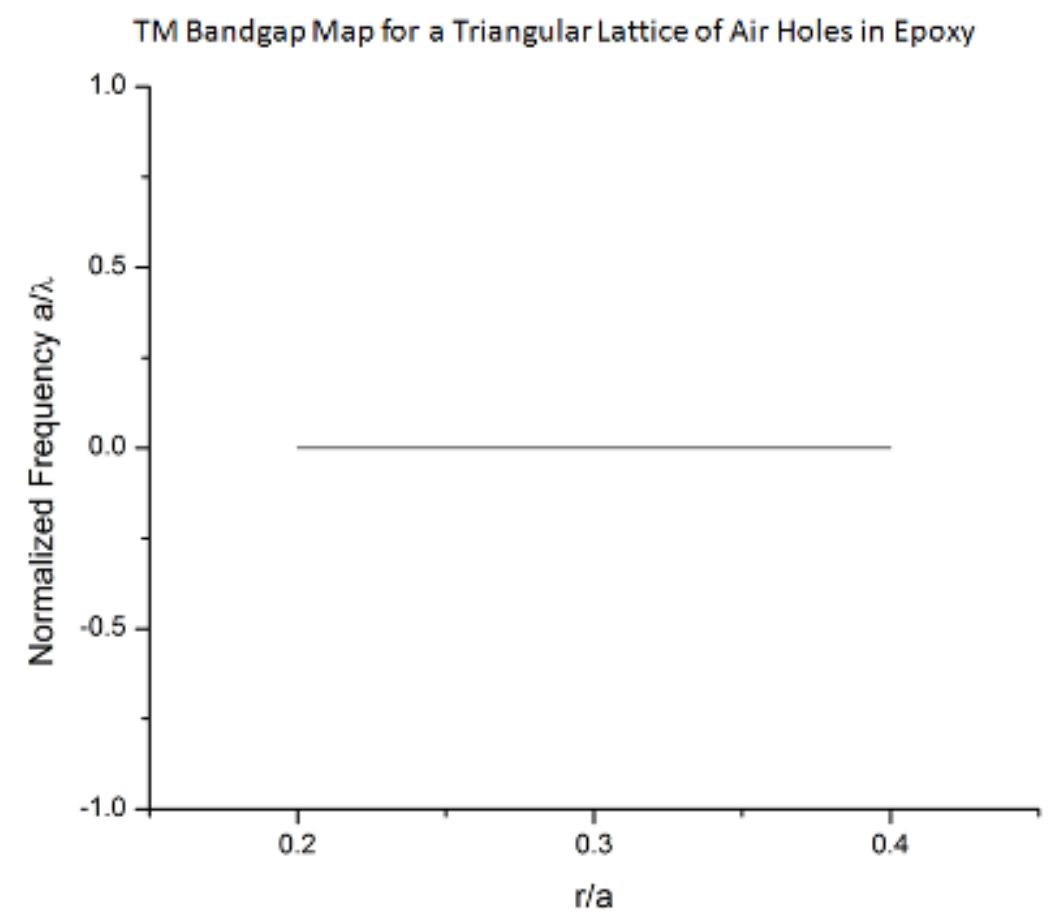

Figure 3.44. TM band diagram of a triangular lattice of air holes in Epoxy. No bandgap was observed for the radii range between $0.2 a$ and $0.4 a$

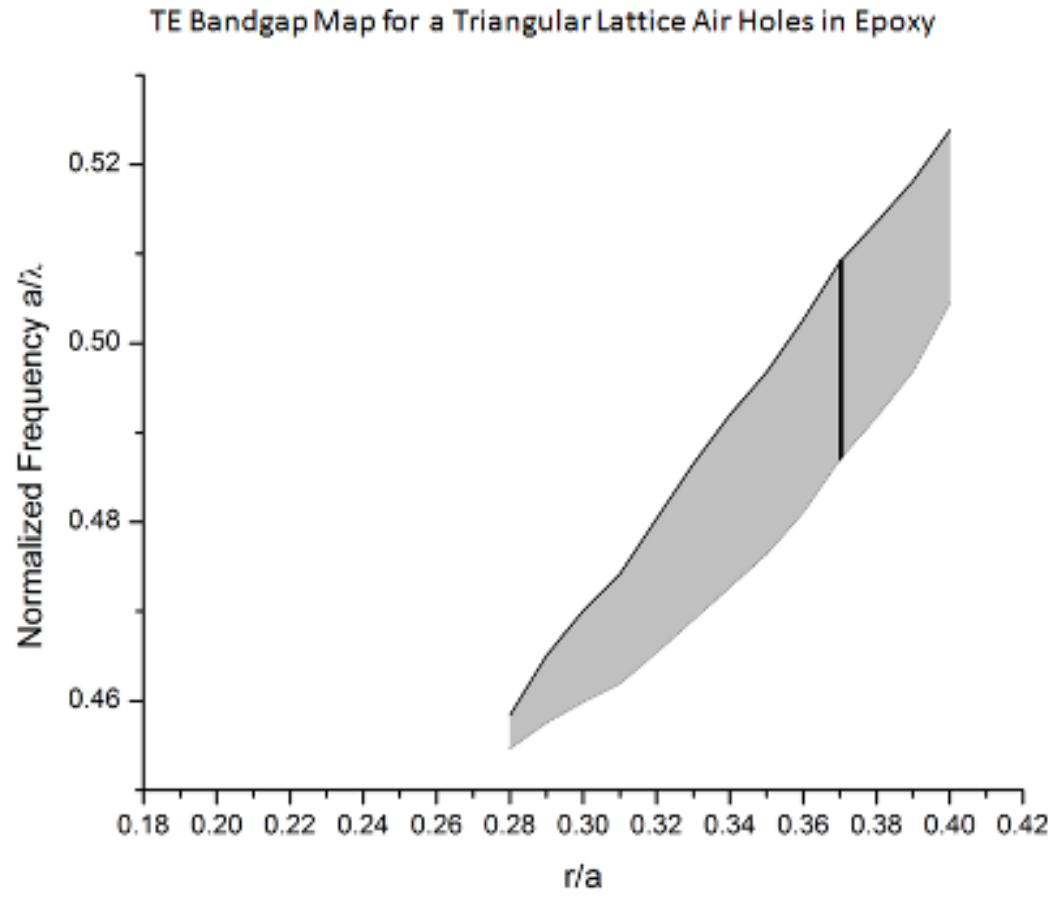

Figure 3.45. TE band diagram of a triangular lattice of air holes in Epoxy. A bandgap of size $4.41 \%$ was observed for a lattice with $\mathrm{r} / \mathrm{a}=0.37$ 


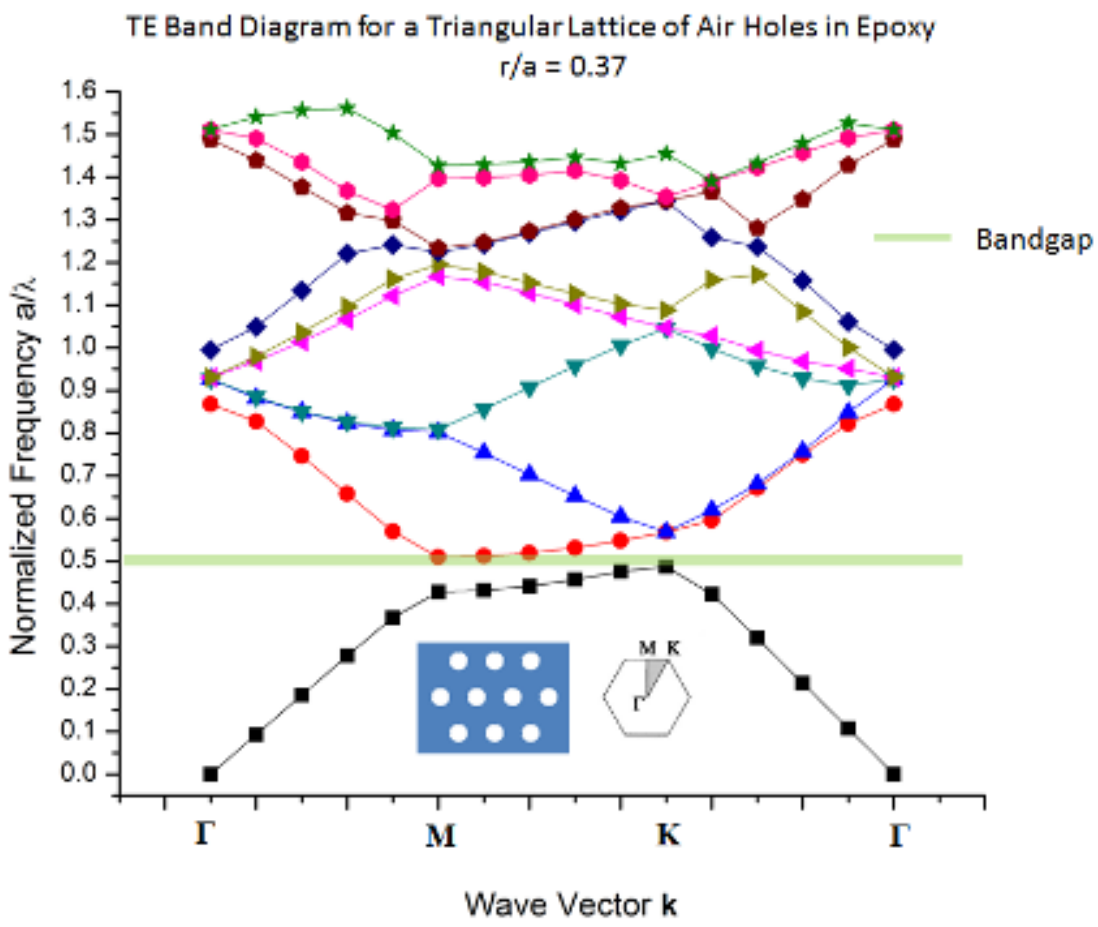

Figure 3.46. TE band diagram for a triangular lattice of air holes in epoxy with r/a $=0.37$

TM Bandgap Map for a Triangular Lattice of Epoxy Pillars in Air

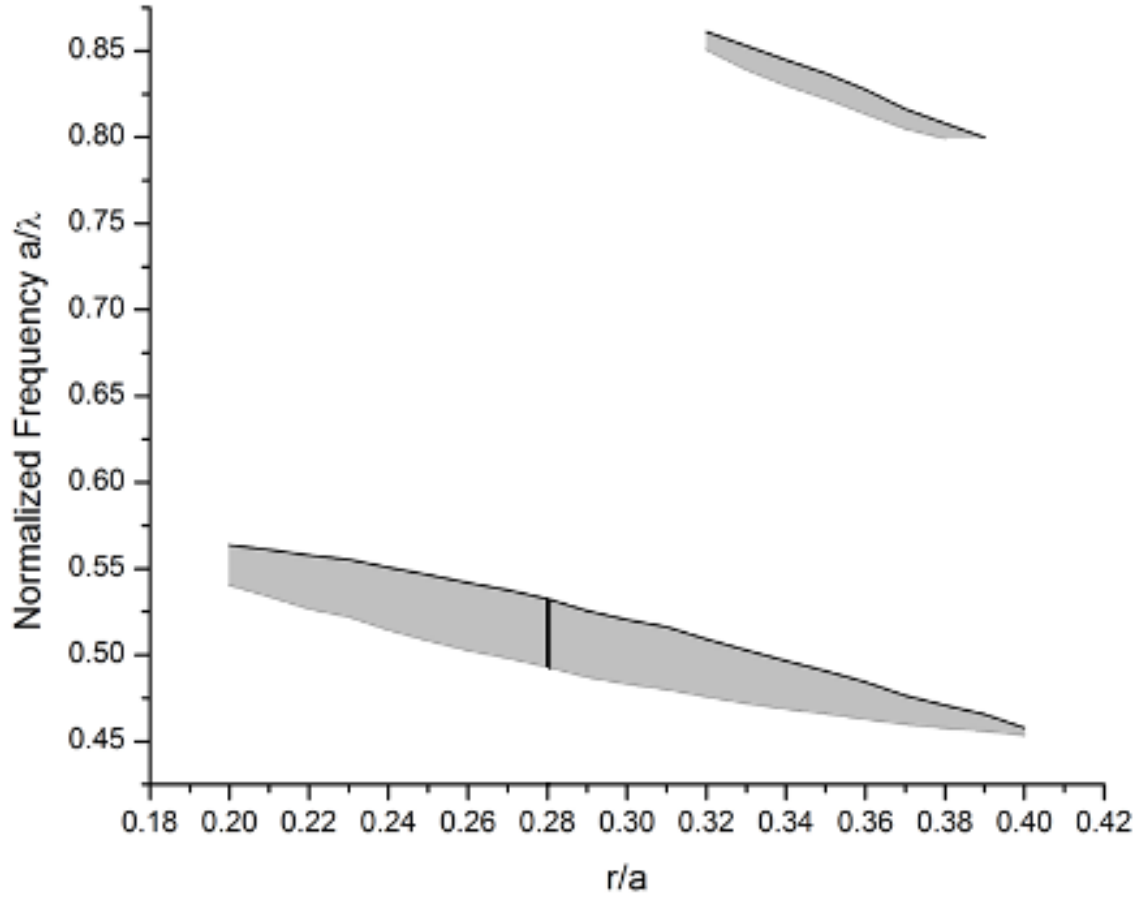

Figure 3.47. TM bandgap map for a triangular lattice of epoxy pillars in air 


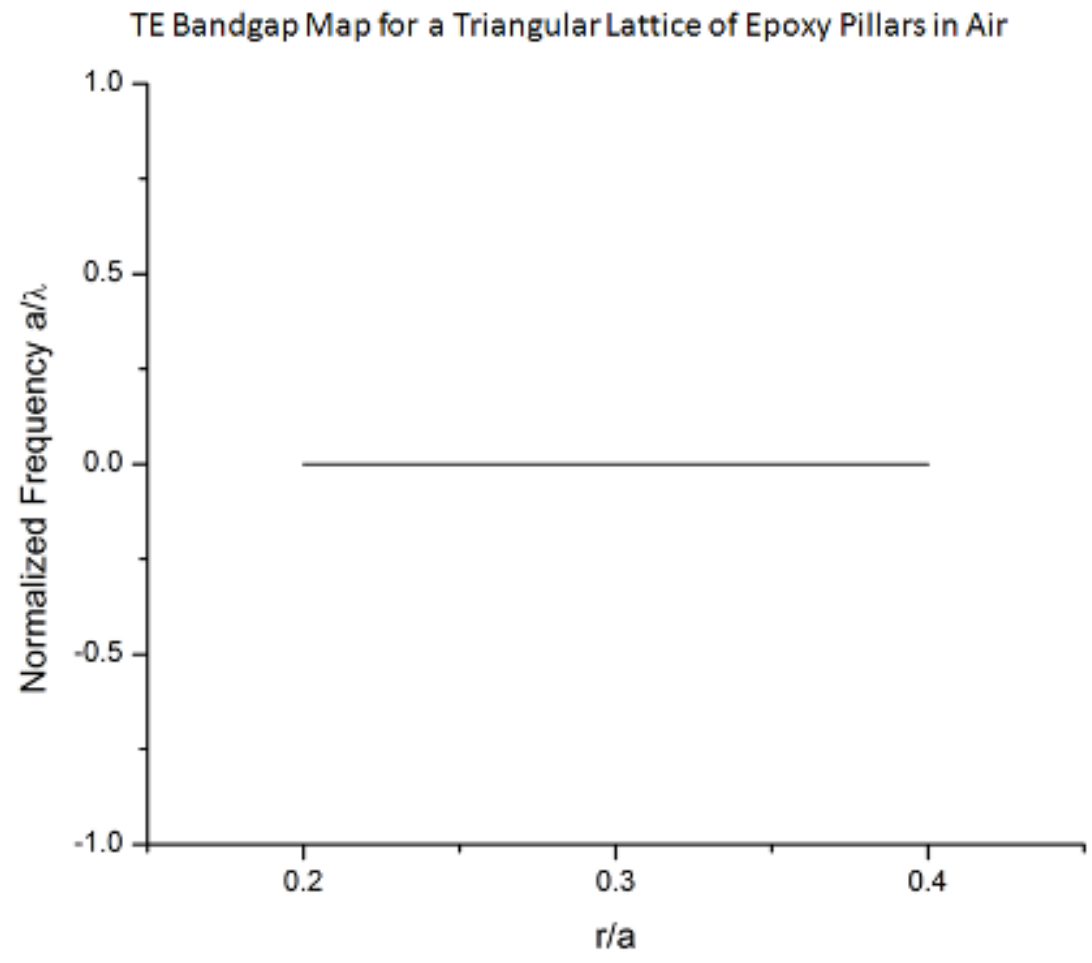

Figure 3.48. TM bandgap map for a triangular lattice of epoxy pillars in air. No bandgap was observed for the radii range $0.2 a$ to $0.4 a$

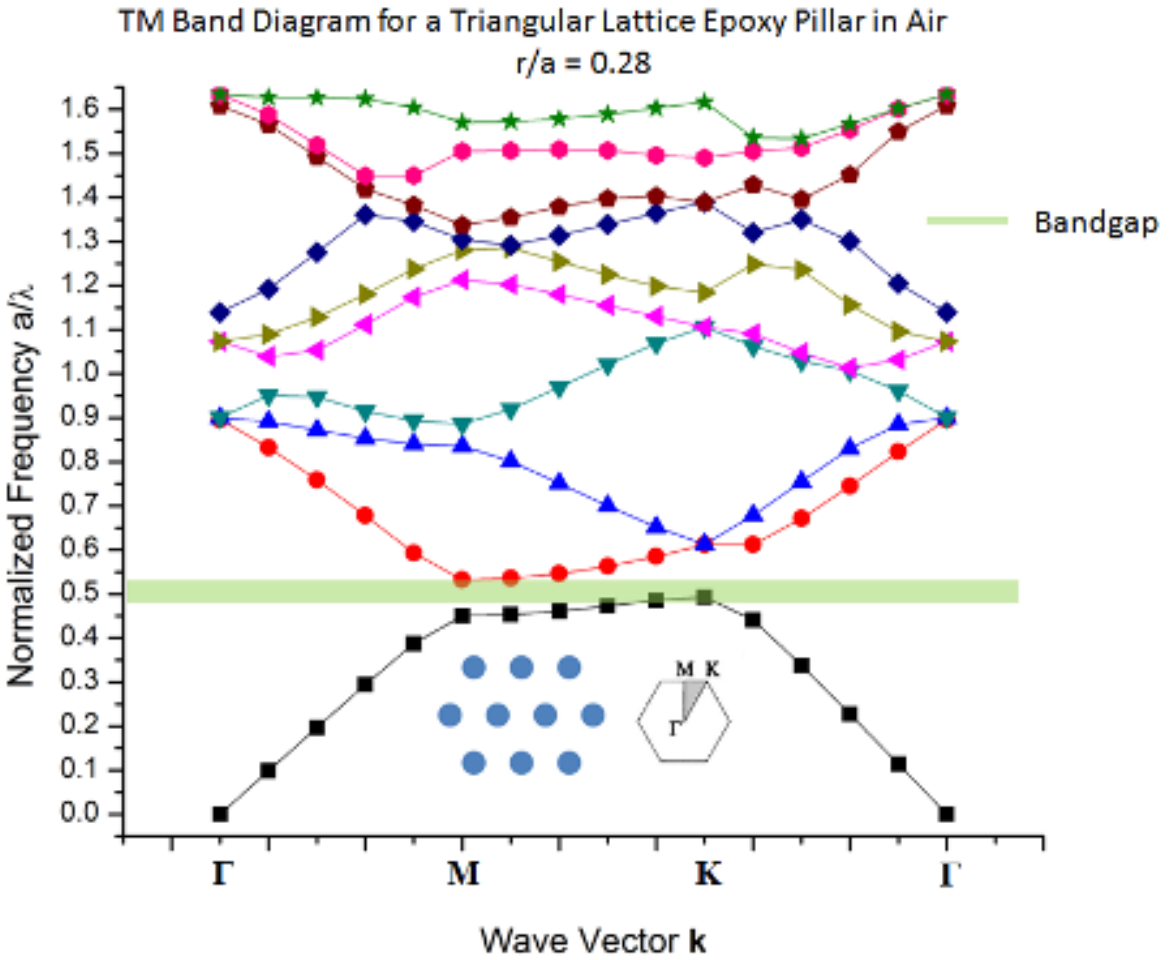

Figure 3.49. TM band diagram for a triangular lattice of epoxy pillars in air with $\mathrm{r} / \mathrm{a}=0.28$ and a bandgap of $7.63 \%$ between the first and second bands 


\subsubsection{Analyzing Infinitely-Thick Photonic Crystal Lattices of Epoxy and Air}

The two lattices that demonstrated partial bandgaps for either the TE or the TM polarizations were:

- Triangular photonic crystal lattice of air holes in Epoxy

- Triangular lattice of Epoxy pillars surrounded by air

If the low dielectric regions in these previous two lattices replaced by that of any material with a refractive index higher than 1, bandgaps will no longer exist. The low refractive index contrast in these lattices prevents the separation of the electric or the magnetic field of the propagating electromagnetic wave in the different regions of the lattice, hence no range of prohibited wavelengths will be created. However, for the two previous lattices, it is still desirable to calculate the actual parameters of these lattices. Here, the IR-emitting quantum dots are replaced with a visible wavelength emission such as that of fluorescein $(515 \mathrm{~nm})$.

For a triangular lattice of air holes in Epoxy, the widest TE bandgap was observed at a lattice with an $r / a$ ratio of 0.37 . Therefore, if the theoretical radius is $0.75 a$, then the actual lattice constant $a$ must be 2.027a. This is simply a scaling factor of the lattice constant. It will allow the lattice to be molded in the nanoscale while at the same time prohibiting the propagation of visible range wavelengths. Table 3.13 summarized the parameters of this lattice.

Table 3.13. Actual lattice parameters of a triangular lattice of air holes in epoxy

\begin{tabular}{|c|c|c|c|c|c|c|c|c|}
\hline \multicolumn{9}{|c|}{ Epoxy - Triangular Lattice of Air Holes in Epoxy } \\
\hline \multicolumn{9}{|c|}{$\mathrm{r} / \mathrm{a}=0.37$ [Scaling: $\mathrm{r}=0.75, \mathrm{a}=2.027]$} \\
\hline r/a Bands & $\begin{array}{c}\text { Gap } \\
\text { Bottom } \\
\text { Frequency } \\
(\mathrm{a} / \lambda)\end{array}$ & $\begin{array}{c}\text { Gap Top } \\
\text { Frequency } \\
(\mathrm{a} / \lambda)\end{array}$ & $\begin{array}{c}\text { Mid-gap } \\
\text { Frequency } \\
(\mathrm{a} / \lambda)\end{array}$ & $\begin{array}{l}\text { Gap } \\
\text { Width } \\
(\%)\end{array}$ & $\begin{array}{c}\text { Desired } \\
\text { Wavelength } \\
\text { (ג) [nm] }\end{array}$ & $\begin{array}{c}\text { Lattice } \\
\text { Constant } \\
\text { (a) }[\mathrm{nm}]\end{array}$ & $\mid \begin{array}{c}\text { Radius } \\
(\mathrm{r}) \\
{[\mathrm{nm}]}\end{array}$ & $\begin{array}{c}\text { Slab } \\
\text { Thickness (h) } \\
\text { [nm] }\end{array}$ \\
\hline \begin{tabular}{|l|l|}
0.37 & 1 and 2 \\
\end{tabular} & 0.487 & 0.509 & 0.498 & 4.408 & 515 & 519.979 & 192.392 & N/A \\
\hline
\end{tabular}

For a triangular lattice of Epoxy pillars in air, the widest TM bandgap was observed at a lattice with an $r / a$ ratio of 0.28 . Therefore, if the theoretical radius is $0.75 a$, then the actual lattice constant $a$ must be 2.678 $\boldsymbol{a}$. The actual lattice parameters are summarized in Table 3.14. 
Table 3.14 Actual lattice parameters of a triangular lattice of Epoxy pillars in air

\begin{tabular}{|c|c|c|c|c|c|c|c|c|c|}
\hline \multicolumn{10}{|c|}{ Epoxy - Triangular Lattice Epoxy Pillars Surrounded by Air } \\
\hline \multicolumn{10}{|c|}{$r / a=0.28[$ Scaling: $r=0.75, a=2.678]$} \\
\hline $\mathrm{r} / \mathrm{a}$ & Bands & $\begin{array}{c}\text { Gap } \\
\text { Bottom } \\
\text { Frequency } \\
(a / \lambda)\end{array}$ & $\begin{array}{c}\text { Gap Top } \\
\text { Frequency } \\
(a / \lambda)\end{array}$ & $\begin{array}{c}\text { Mid-gap } \\
\text { Frequency } \\
(a / \lambda)\end{array}$ & $\begin{array}{c}\text { Gap Width } \\
(\%)\end{array}$ & $\begin{array}{l}\text { Desired Wavelength } \\
(\lambda)[\mathrm{nm}]\end{array}$ & $\begin{array}{l}\text { Lattice } \\
\text { Constant } \\
\text { (a) }[\mathrm{nm}]\end{array}$ & $\begin{array}{l}\text { Radius } \\
\text { (r) }[\mathrm{nm}]\end{array}$ & $\begin{array}{c}\text { Slab } \\
\text { Thickness } \\
\text { (h) }[\mathrm{nm}]\end{array}$ \\
\hline 0.28 & 1and2 & 0.493 & 0.532 & 0.512 & 7.631 & 515 & 707.509 & 198.102 & $\mathrm{~N} / \mathrm{A}$ \\
\hline
\end{tabular}




\section{CHAPTER 4: FABRICATION OF SEMICONDUCTOR- AND POLYMER- BASED PHOTONIC CRYSTAL LATTICES}

\subsection{INTRODUCTION}

In the previous chapter, the modeling tools used to predict the behavior of electromagnetic waves as they propagate through the periodic photonic crystal lattices were discussed. These tools provided the critical photonic crystal dimensions that must be known in order to fabricate a lattice that can potentially manipulate the flow of specific desired wavelengths. Tables summarizing the actual dimensions of the lattices indicate that they all possess sub-micron dimensions. Therefore, photolithography processes can no longer be used due to the diffraction limit of light at these sub-micron scales. Instead, E-beam Lithography (EBL) is used to fabricate the nano-scale structures. The process to fabricate these nanoscale structures starts with an e-beam lithography step, but requires a series of subsequent steps involving metal mask deposition, lift-off, etching, etc.

Throughout this chapter, the EBL process as well as the subsequent steps of metal deposition and plasma etching is first explained. Afterwards, the developed recipes to fabricate nano-scale photonic crystal lattices in Silicon, Silicon on Insulator (SOI), PDMS, and Epoxy are discussed.

\subsection{ELECTRON-BEAM LITHOGRAPHY}

The term 'lithography' in semiconductor science is used to refer to the microfabrication processes that involve the use of ultraviolet light, or photons, to expose a photo-sensitive material called photoresist and create a desired pattern that can be transferred to an underlying substrate in subsequent fabrication steps [52-54]. The range of wavelengths that are used to do this exposure is normally between 193 and $436 \mathrm{~nm}$. At length scales comparable to these wavelengths, however, the diffraction limit of conventional optics becomes an issue, making patterns at such scales difficult produce with optical lithography [52-54].

E-beam Lithography is a very precise, high-resolution patterning method capable of overcoming the previous diffraction limit of photolithography and produce structures on a scale down to $1 / 100$ of a visible photon wavelength $[55,56]$. Unlike photons, electrons' associated waves have wavelengths of very few angstroms, which are several orders of magnitude shorter than the optical wavelengths. The low mass of electrons allows them to penetrate a resist layer to dissipate their energy and then become scattered in a grounded substrate. Therefore, when the EBL techniques first emerged, it was predicted to replace photolithography in VLSI manufacturing when PMMA (Poly Methyl Methacrylate) was first introduced 
as an electron-beam resist. However, the low throughput of this method compared to regular photolithography prevented it from being fully integrated into the mass IC production.

Unlike photolithography, the EBL process can be done without the need of any actual physical photomasks. Its 'direct write' characteristic allows the creation of the desired pattern in a computer aided design (CAD) file. Software control over the EBL hardware components translates this designed CAD pattern into a writing 'path' where the electron beam scans the surface as designed by the file. The extremely high resolution of this method and the direct write flexibility together allowed the method to become an initial tool for nano-scale patterning. Both photolithography and EBL are designed to control particles, photons and electrons respectively, and focus them to a very concentrated energy field for writing. Therefore, it is fair to say that there are a lot of similarities between the two methods in their optical components. EBL systems use electrostatic and magnetic lenses to focus its electron beam. These lenses operate to deflect electrons and slow their speed as they penetrate through different electromagnetic fields. The source of electrons comes from the electron gun which is made of a cathode and a lens. The electrons are produced by the cathode and then focused by the lens to become a beam.

The main components of an EBL system's column are demonstrated in Figure 4.1. The electron gun is one of the main components as it is the source of the electrons. These electrons are normally accelerated to a kinetic energy between $10 \mathrm{keV}$ and $100 \mathrm{keV}$ [57]. If the gun cathode component is of a field emission type, this portion is normally designated a separate continuous vacuum system to maintain the lowest number of gaseous particles before the beam is released and hence reduce beam deflection at that early stage. Once the beam is created, the gun alignment system is then assembled along a 1 meter vertical column that is very precisely designed to ensure a well defined beam when reaching the sample. The condenser lens is the first of these alignment components. Its main purpose is to converge and condense the emitted electron beam from the cathode. The beam blanker is placed next in the path of the beam to allow control over the switching mechanism of the beam when it is required to expose only specific regions at specific times. This blanking mechanism is not mechanical as its name may indicate. Instead, it is simply an electrostatic deflector that deflects the beam off its axis when it is desired to not expose a specific region. Zoom lens then adjusts the focal plane followed by astigmatism correction of the beam that is done by the Stigmator [57].

Once the beam is aligned and focused, apertures then allow control over its width. The smaller the angle which the beam makes after the aperture the less spread out the beam is. Smaller angles also produce better electron optical resolution. Therefore, small apertures are normally used when high resolution writing is required. After passing through the aperture, the beam is then focused by the last lens 


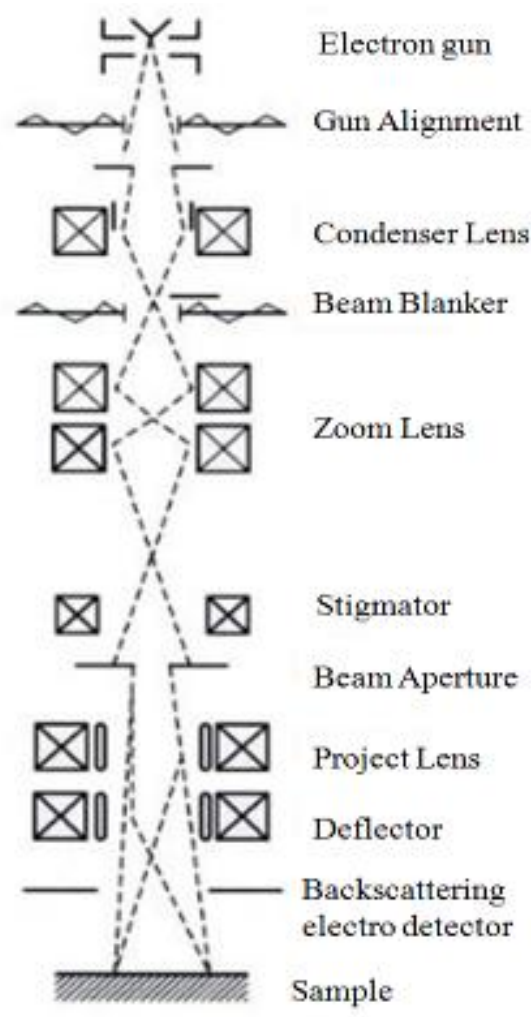

Figure 4.1. The electron optical components inside the column of an EBL system [57]

component of the column which is the Projection lens. Electrostatic or magnetic deflectors are then placed to allow for control of the beam deflection when electrical signals are produced that correspond to a specific pattern [57].

When performing an EBL procedure, several parameters must be set to allow for a high resolution pattern. The most important one is the beam spot size as it determines the resolution of the overall pattern and the smallest exposed features that can be created. The acceleration voltage is another parameter that must be set according to the chosen e-beam resist and its thickness. Higher acceleration voltages are normally used to produce higher resolution features in thick resists as the proximity effect is reduced. The beam current must also be set carefully. It controls the speed of the lithography process and therefore, higher beam currents are capable of exposing more features at a given time. However, the beam current value is normally increased when high throughput is desired but smaller beam current values are usually desired as slower exposure can produce higher resolution patterns. Finally, the scanning field is the parameter that determines the largest area that can be written without the need to stitch several portions of the desired pattern together. 


\subsection{ELECTRON-BEAM EVAPORATION}

E-beam evaporation is a technique of depositing thin materials on a substrate through the use of a high energy electron beam that causes these materials to evaporate. A wide variety of materials such as: refractory metals, low-vapor-pressure metals, alloys, and oxides can be evaporated using this technique $[57,58]$. The method concentrates an electron beam in a crucible containing the desired material to be deposited, which creates a large amount of heat capable of evaporating the target and producing high deposition rates. This method has advantages over thermal evaporation techniques including lower temperature processing. High temperatures are often undesirable due to contamination and the effects of high temperatures on lift off processes involving e-beam resists.

The main components of an e-beam evaporation system are demonstrated in Figure 4.2. These include a tungsten thermionic electron emitter, an electromagnetic coil steering the electron beam by producing a transverse magnetic field, a high voltage DC power supply, and a crucible or hearth where the target material is kept [58]. When a high voltage is applied to the tungsten filament, electrons are generated thermionically from the filament. These electrons are emitted in a random manner and therefore they must be directed in a specific path in order for the evaporation of the target material to take place. Therefore, a deflection/focusing of this beam is achieved when the electromagnetic coils generate a magnetic field capable of shaping the path of the beam. The electron beam then bombards the source material, transferring its kinetic energy to heat upon impact. However, it is important to point out that the energy given off by a single electron upon impact is very small, which is not sufficient to produce the desired heat. Instead, this heat is generated due to the large number of focused electrons hitting the surface continuously making the total energy sufficient to melt and evaporate the target.

The power supply voltage for this procedure is typically between 10 to $30 \mathrm{kV}$ with a power ranging from 10 to $30 \mathrm{~kW}$ and it is always carried out under a vacuum of $10^{-5}$ torr or less. This high vacuum ensures minimal chamber contamination and prevents gas collisions between evaporated molecules and other particles in the chamber that will severely affect the smoothness of the deposited material. Moreover, to prevent the filament coating from getting contaminated, the filament is typically located out-

of-sight of the evaporated material. Source materials are held in a $\mathrm{Cu}$ hearth, which is water cooled to prevent its outgassing, melting, and alloying with molten deposition material [58]. 


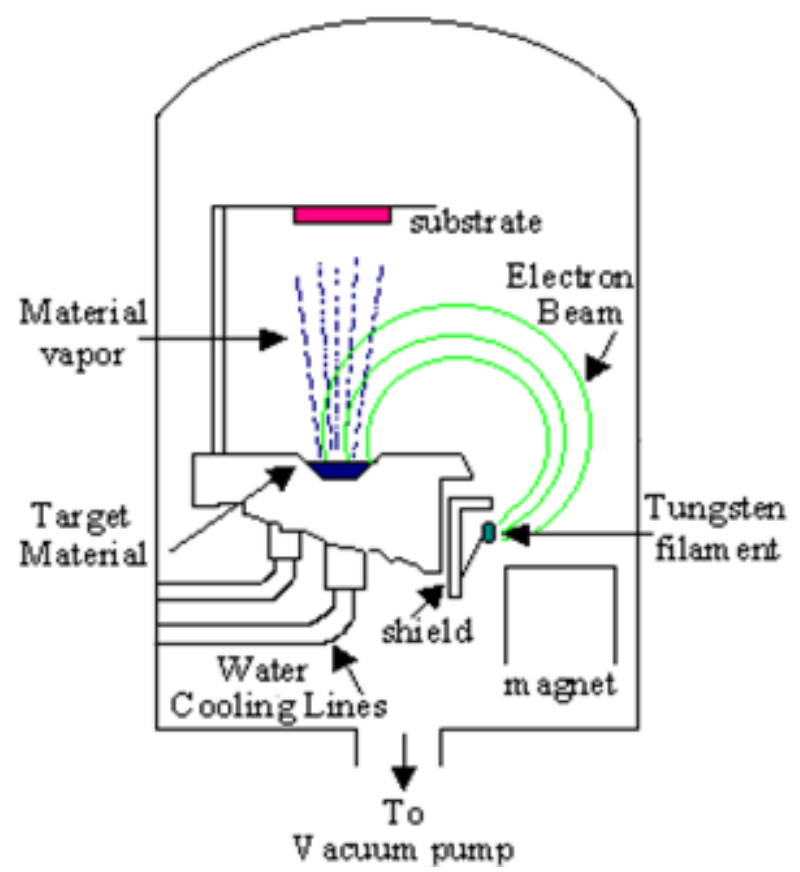

Figure 4.2. E-beam Evaporation system components [59]

\subsection{PLASMA ETCHING}

Early Integrated Circuit (IC) manufacturing was highly dependent on wet etching techniques due to their high selectivity, simplicity, and low cost. Selectivity is a measure of the etch depth in a desired substrate to its mask for a fixed period of time (normally one minute). However, the isotropic etching profiles and undercutting of the mask in wet etching techniques imposed critical limitations to device yield and reliability. Moreover, as IC manufacturing pushed toward more dense chips incorporating submicron structures, accurate transfer of the mask pattern to the underlying substrate material becomes a crucial step that wet etching techniques failed to provide $[40,60]$. To overcome these limitations, plasma etching was introduced as an efficient technique capable of providing precise control over aspect ratio, selectivity, and uniformity of the etched surfaces [60].

A plasma reactor, illustrated in Figure 4.3, consists of two separated electrodes. One of these two electrodes can be the interior of the etching chamber. RF power is used in the majority of the plasma etching systems instead of DC power to ignite the etching plasma is mainly to prevent any possible charge accumulation when dielectric samples are used [61]. If a sufficient high frequency voltage is applied between the two electrodes, an electric field is generated, and when a specific gas is introduced into the 

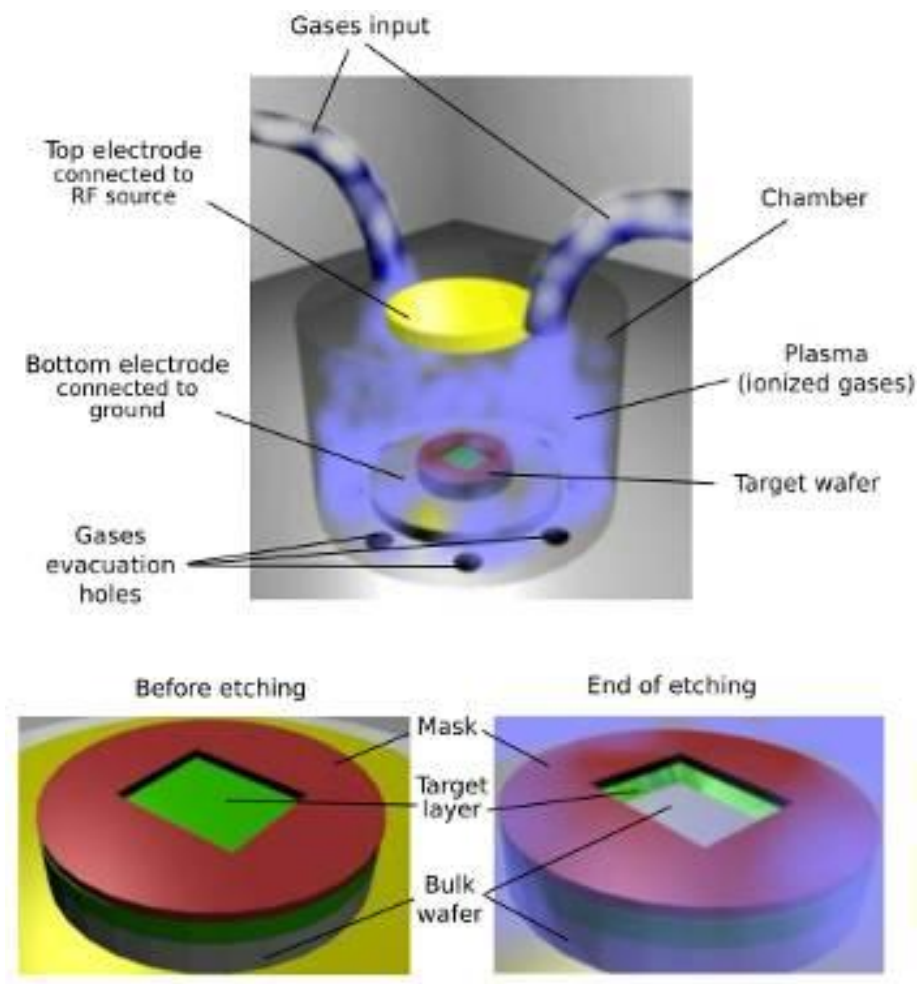

Figure 4.3. Schematic of plasma etching [61]

chamber, plasma can be generated. Plasma is a partially ionized gas that produces an obvious glow when discharging inside the chamber. Therefore, if a sample happens to be placed inside the same chamber, as shown in Figure 4.3, the uncovered region of the sample surface becomes exposed to the reactive species of the plasma and etching of the surface takes place [60].

The four basic methods of plasma etching are:

- Chemical Plasma Etching

- Sputtering Plasma Etching

- Reactive Ion Enhanced Etching

- Protective Ion-Enhanced Etching.

In the chemical based etching, thermalized neutral radicals chemically combine with molecules of the substrate's top surface to generate volatile products that can be removed from the sample. The gas mixture in this method is chosen to generate specific reactive species [62]. This method provides good etch rates and minimizes the plasma-induced damage. However, it is not a directional etching technique and isotropic etch profiles are commonly observed when this method is used. In Sputtering-based plasma 
etch, energetic ions formed in the plasma are accelerated by the applied field toward the sample surface to cause a physical bombardment that etches the exposed surface. This method produces anisotropic profiles but it can also result with damaged or rough surfaces due to the physical bombardment effect that it involves [62].

In the Energetic-Ion-Enhanced mechanism, a combination of particles from the previous two methods is generated to produce etch rates that exceed those of chemical and sputtering rates. It is normally referred to as Reactive Ion Etching (RIE) [62]. The main advantage of this mechanism is that it results with highly anisotropic etch profiles and can be used to remove polymer by-products [62].

Finally, in Protective Ion-Enhanced Plasma Etching, an inhibitor film coats the inner walls of the etched features to generate a layer that blocks the etching of the sidewall to lead to an increased anisotropy. This mainly happens due to the fact that the generated plasma normally contains neutral etchant species that adsorb to surface of the sample continuously. As etching takes place, ions remove the inhibitor from the horizontal surfaces leaving behind the protective film untouched to result with highly anisotropic etch profiles [62].

\subsection{SILICON-BASED PHOTONIC CRYSTALS}

The main tools that were used to fabricate photonic crystal structures in silicon were explained in the earlier sections of this chapter. These tools were used in a specific sequence in order to fabricate the final photonic crystal structure in silicon. A top-down approach was followed for most of the processes that will be discussed in this section. This method started by first creating a DesignCAD file using the Nanometer Pattern Generation System (NPGS) software tool that controlled the behavior of the e-beam lithography system [63]. The modeling results discussed in Chapter 3 helped in generating this DesignCAD file containing the desired photonic crystal lattice dimensions that posses a photonic bandgap. Negative or positive tone e-beam resists were then spin-coated the top surface of the sample with desired thicknesses as different spin speeds correspond to different thicknesses. The patterns were then written in this top e-beam sensitive polymer layer and then developed before several other metal mask deposition, lift-off, and etching steps took place to produce the final structures that could be optically characterized.

Two methods will be explained in detail in the next two sections. The first is a recipe that is adapted from [43] to produce suspended photonic crystal lattices of air holes in Silicon by starting first with a negative tone e-beam resist, ma-N 2403. The second will be the opposite of the first, which is to create silicon rods (pillars) surrounded by air using a positive tone resist, PMMA 950K A3. Exposure and spin recipes have been already developed in [43]. However, optimizing these recipes to the Silicon-on- 
Insulator and regular Silicon samples will be discussed in detail. SEM as well as descriptive images of each fabrication step along the process from end to finish will be demonstrated.

The fabrication processes explained in the following two sections involve several steps and lift off processes that can affect negatively on the overall quality of the final fabricated structures. Therefore, in order to minimize the number of the fabrication steps to produce final $\mathrm{PhC}$ structure in silicon and improve the side-wall quality of these structures, a negative tone resist Hydrogen-Silsesquioxane is used. This resist can serve as a hard etching oxide mask once treated with Oxygen plasma.

\subsubsection{Fabrication of Symmetric Photonic Crystal Lattices of Air Holes in Silicon}

In order to fabricate symmetric photonic crystal lattices, commercially available Silicon-on-Insulator wafers were used. These wafers are made of a bulk silicon substrate and a thin top silicon layer that sandwich an approximately $1-\mu$ m layer of smooth $\mathrm{SiO}_{2}$. Figure 4.4 demonstrates a tilted cross section image of one of these wafers.

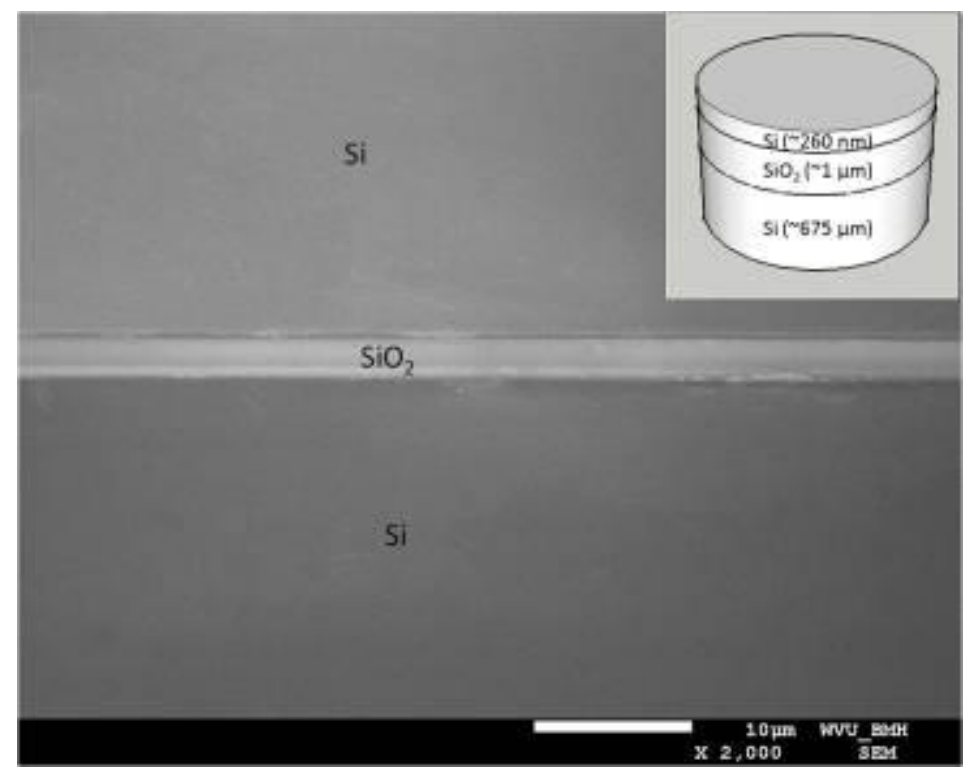

Figure 4.4. A tilted SEM image demonstrating the different layers of an SOI wafer. Top right corner inset demonstrates a description of the thickness of each layer

The symmetry was achieved when the final photonic crystal structure was fabricated in the top silicon layer and the underlying sacrificial $\mathrm{SiO}_{2}$ layer was etched away. This created a channel of air below the slab that could be filled with any other liquid material when flow measurements were carried out. The process to create these symmetric structures is quite complex and involves several steps. This is mainly due to the fact that the thin e-beam resists used in these processes have poor dry etch resistance 
and very poor selectivity when used alone as masks to etch Si. Therefore, metal mask deposition and liftoff processes were done to achieve a high enough depth in the Si substrate upon etching.

The fabrication started with a regular degreasing (cleaning) step in which the sample were sonicated for 5 minutes in Acetone and then immersed in Methanol for another 5 minutes. The sample was then rinsed with DI water for 5 minutes and then dried with a steady Nitrogen flow produced by a nitrogen gun. The sample was then put in an oven set $120^{\circ} \mathrm{C}$ for 20 minutes for a dehydration step. At this time the sample surface was ready to be coated with the e-beam resist. In order to produce holes in silicon, a negative tone e-beam resist was used to create pillars in the resist. This is simply due to a later lift-off process that was carried out to create the inverse of that to be transferred to the underlying substrate.

The negative tone e-beam resist selected for this process was ma-N 2403 purchased from Micro Resist Technology. This resist has an excellent thermal stability with pattern resolution down to $50 \mathrm{~nm}$. It is also very easy to remove and can be exposed in a range of energies between $20-50 \mathrm{keV}$ [64]. The negative-tone behavior of this resist is explained in Figure 4.5.
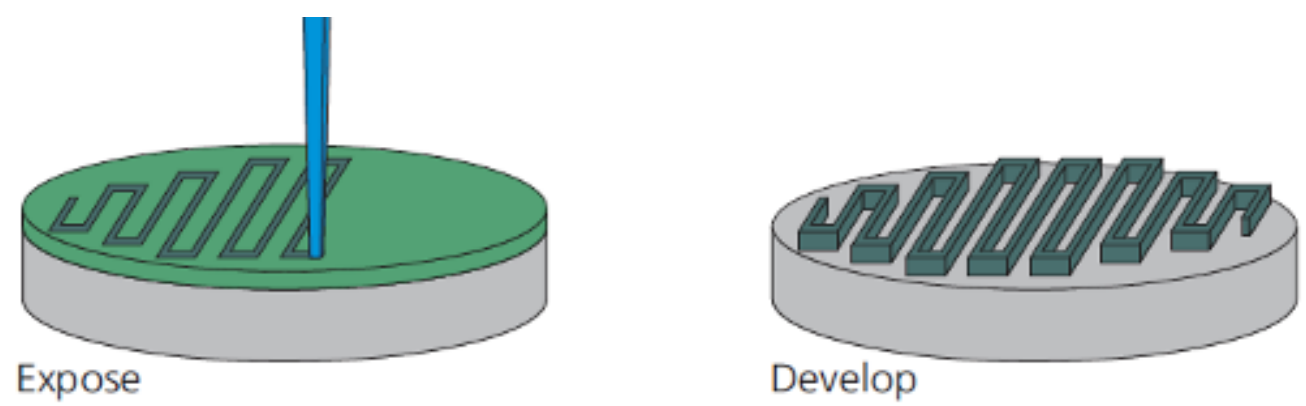

Figure 4.5. The negative-tone e-beam resist pattern development mechanism [64]

The ma-N 2403 was spun on the wafer for 50 seconds at $5000 \mathrm{rpm}$. A soft bake step was then done to remove solvents and make the resist more sensitive to the electron beam. This baking step was done on a hot plate set to $95^{\circ} \mathrm{C}$ for 3 minutes to achieve a final thickness of approximately $240 \mathrm{~nm}$ on top of the $\mathrm{Si}$ sample. The EBL process then began. A JEOL 7600 SEM system with e-beam lithography capability was used to accomplish this step.

The pattern created in DesignCAD with submicron lattice dimensions was loaded to the NPGS software and converted into a run file that controlled the deflectors and the beam blanker by sending electric signals to the SEM. A detailed explanation of pattern preparation using the DesignCAD tool and the e-beam lithography procedure is explained in Appendices A and B, respectively. Once the beam was focused, the writing of the pattern was done at a dose range between $58-64 \mu \mathrm{C} / \mathrm{cm}^{2}$. This was the dose range that was found to be optimal to produce the best patterns on SOI wafers. The EBL write was then 
followed by an immediate development step in ma-D 525 for 65 seconds without agitation. To prevent overdevelopment from happening, the sample was then immediately immersed in water for at least 30 seconds and then dried with a slow flow of $\mathrm{N}_{2}$ produced by a regular Nitrogen gun.

At this stage, the sample contained a pattern that could easily be observed in an optical microscope. Submicron features, of course, were not visible. However, an idea of how well the development/exposure processes could be examined based on the shape of resist edges. The pattern was a two dimensional photonic crystal lattice of ma-N pillars surrounded by air, which is the inverse of what the desired final $\mathrm{PhC}$ lattice. Therefore, a thin layer of Ni was deposited using a Temescal BJD 2000 E-beam Evaporator to a thickness of approximately $30 \mathrm{~nm}$. Nickel was selected due to the high $\mathrm{Ni} / \mathrm{Si}$ etching selectivity which was approximately 1:15 at a $150 \mathrm{~W}$ RIE power [40]. Therefore, very thin layers of Ni were capable of withstanding prolonged dry etching procedures and achieved depths of several hundreds of nanometers in $\mathrm{Si}$.

The sample was then immersed in Remover PG (MicroChem ${ }^{\circledR}$ ) set to $65^{\circ} \mathrm{C}$ for 15 minutes followed by a two -second sonication step ensure the full removal of the polymer. The Remover PG removed the ma-N polymer pillars and therefore lifted off the deposited layer of $\mathrm{Ni}$ on top of them. This created holes in the thin Ni layer which was used as an etching mask when the pattern was transferred to the underlying thin silicon layer. The etching procedure was done using a $\mathrm{CF}_{4} / \mathrm{O}_{2}$ gas mixture plasma using a MinilockPhantom III ICP-RIE system. The etching parameters were: $400 \mathrm{~W}$ ICP power, $150 \mathrm{~W}$ RIE power, 10 mTorr chamber pressure, $27 / 3 \mathrm{sccm}-\mathrm{CF}_{4} / \mathrm{O}_{2}$ gas mixture flow rate ratio, and $-130 \mathrm{~V} \mathrm{DC}$ bias. One minute of etching using these previous parameters was sufficient to create $\sim 300 \mathrm{~nm}$ holes in Si. The unetched Ni layer was then removed by dipping the sample in a Ni-etchant for 30 seconds with minor agitation. The sample was then cleaned in DI water and prepared for the final fabrication step.

In the last step, the symmetry was created but dipping the sample in a Hydrofluoric Acid solution (HF) for 15 seconds. The sample was then rinsed in DI water for 5 minutes and dried very gently with a Nitrogen gun. The HF here wet etched the underlying $\mathrm{SiO}_{2}$ sacrificial layer and created a channel-like structure with the photonic crystal structure being the ceiling of this channel. SEM and descriptive drawings of this fabrication procedure are demonstrated in Figures 4.6-4.8. The actual dimensions of the fabricated structures compared to the as-designed dimensions are summarized in Table 4.1.

Figure 4.7 and 4.8 demonstrate SEM images of successfully and perfectly fabricated structures when the previous process is followed precisely. Several inaccuracies can, however, result with collapsed structures or even unresolved photonic crystal features. For example, under-development of the resist, depositing thick layers of the Ni mask, or over sonicating the sample after the Remover PG step, can all potentially cause a bad lift off result in which the ma-N pillar either do not lift off or the inverse structure in $\mathrm{Ni}$ is broken due to the over sonication. SEM images demonstrating these two cases are shown in 
Figures 4.8 and 4.9. Moreover, blow drying the suspended samples after the HF step with a fast Nitrogen gas flow can severely cause the suspended 260-nm-thick structures to collapse. Figure 4.10 demonstrates an example of this behavior as well.

Table 4.1. The as-designed vs. as fabricated dimensions of the $\mathrm{PhC}$ in silicon. A deviation of only $2.84 \%$ was observed

\section{Triangular Lattice of Holes in Silicon $(r / a=0.35)$}

\begin{tabular}{|c|c|c|c|c|c|}
\hline \multicolumn{3}{|c|}{ As Designed Dimensions } & \multicolumn{3}{|c|}{ As Fabricated Dimensions } \\
\hline$d$ & a & $\mathrm{h}$ & $d$ & a & $\mathrm{H}$ \\
\hline $208 \mathrm{~nm}$ & 297.5 & $260 \mathrm{~nm}$ & $214 \mathrm{~nm}$ & $297.5 \mathrm{~nm}$ & $260 \mathrm{~nm}$ \\
\hline
\end{tabular}
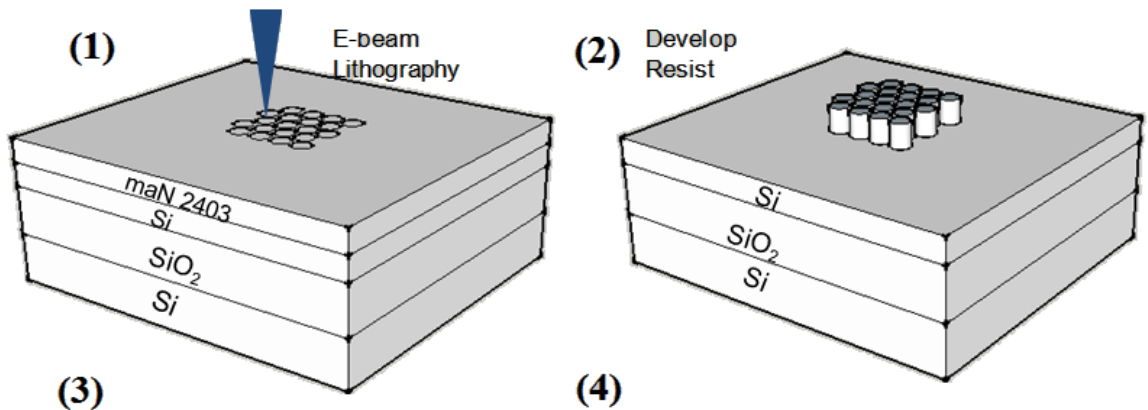

(4)
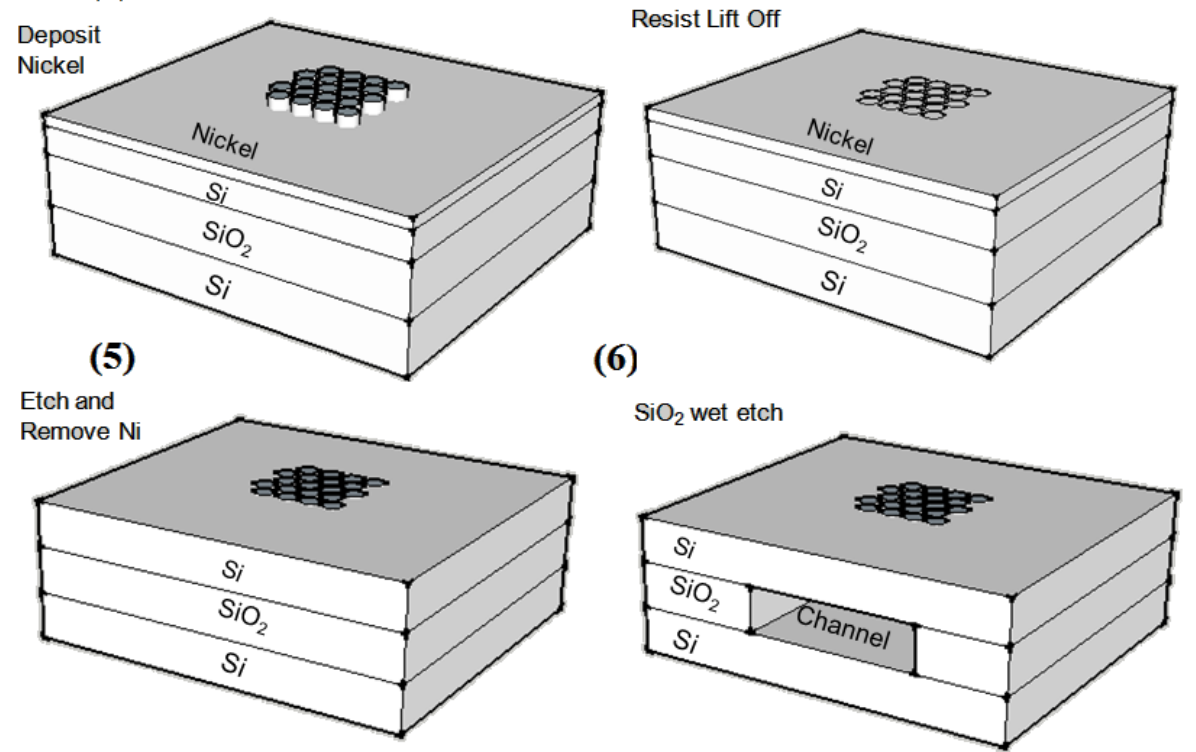

Figure 4.6. Process flow of a suspended $\mathrm{PhC}$ in silicon as adapted from [43]. (1) ma-N 2403 was first spun coated on the SOI sample and an EBL procedure was carried out to define a pattern in the resist. (2) After developmen in ma-D 525 for 65 seconds, pillars of ma-N were resolved. (3) A thin layer of $\mathrm{Ni}$ was then evaporated on the surface. (4) A lift-off process was then done to obtain the desired pattern in the Ni hard mask. (5) $\mathrm{A} \mathrm{CF}_{4} / \mathrm{O}_{2}$ plasma etch was then carried out to transfer the pattern to the underlying Si layer. (6) A 15-second dip in HF solution was then done to open the underlying channel and achieve the desired symmetry above and below the slab. 


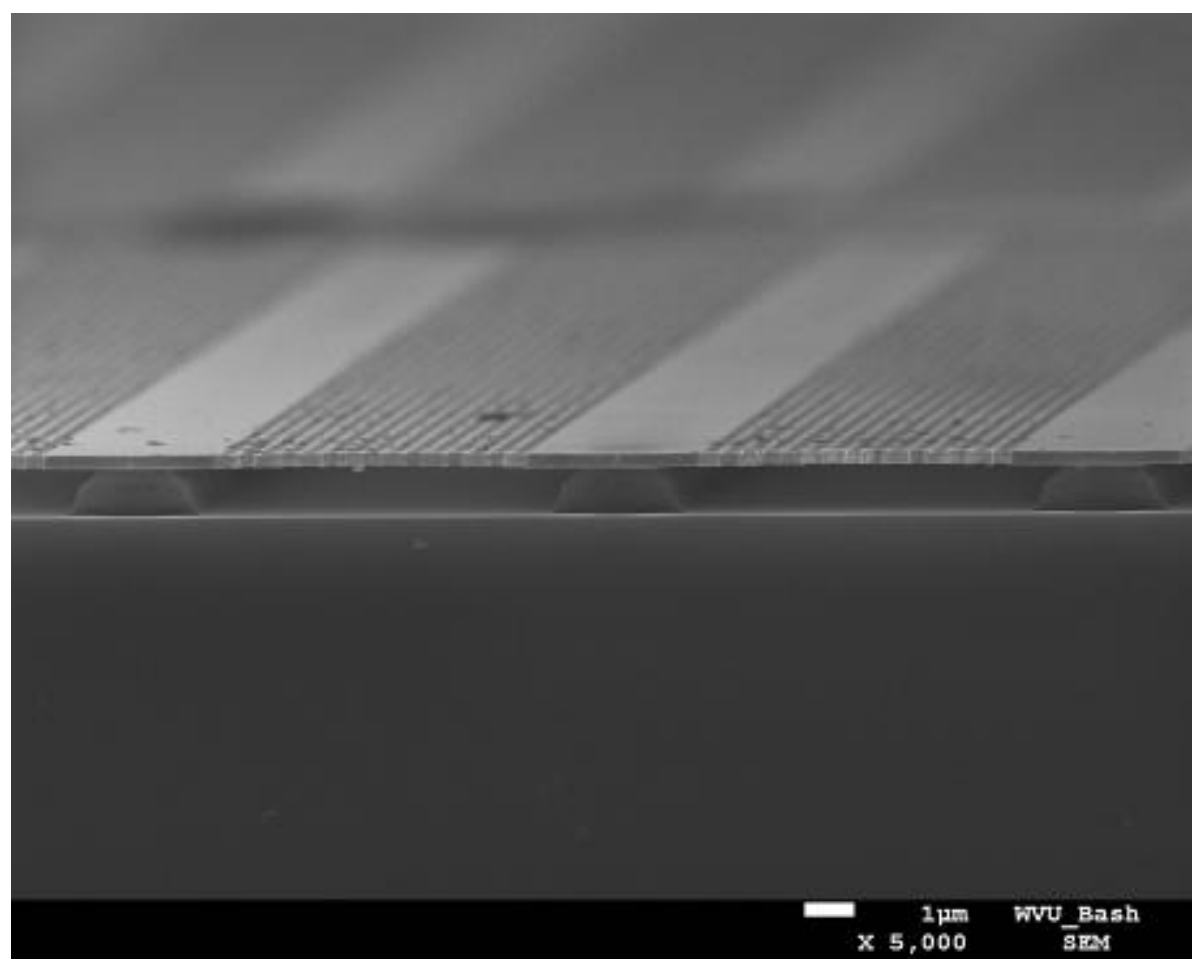

Figure 4.7. SEM image of the suspended PhC structures in silicon with underlying air channels. Image was captured at $85^{\circ}$ glancing angle

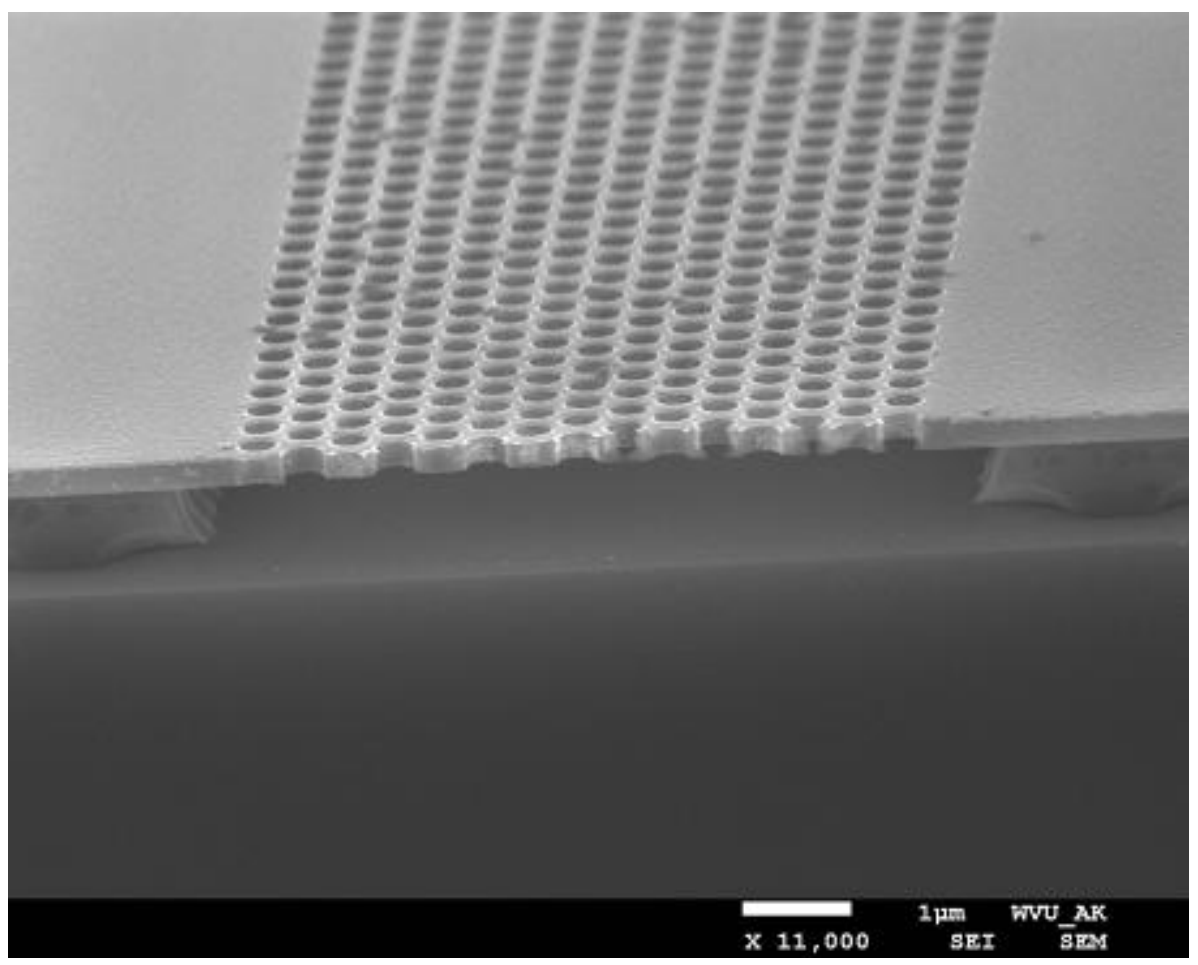

Figure 4.8. A high magnification image of the $\mathrm{PhC}$ structure in silicon and the underlying channel. Image was captured at $80^{\circ}$ glancing angle 


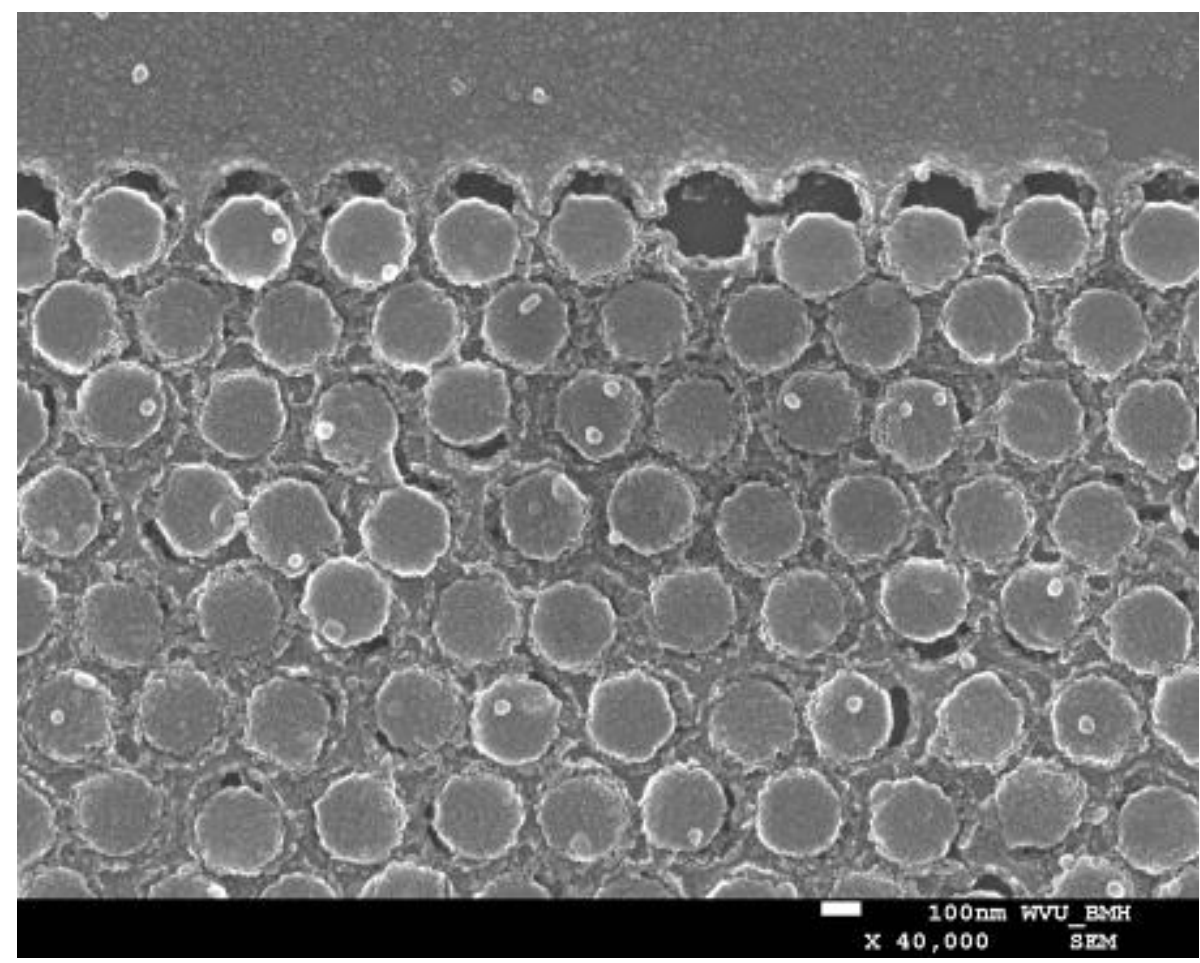

Figure 4.9. Thick Ni layers can cause serious problems when trying to do the lift off process.

In this image, the Ni remained on top of the ma-N pillars even after a sonication step.

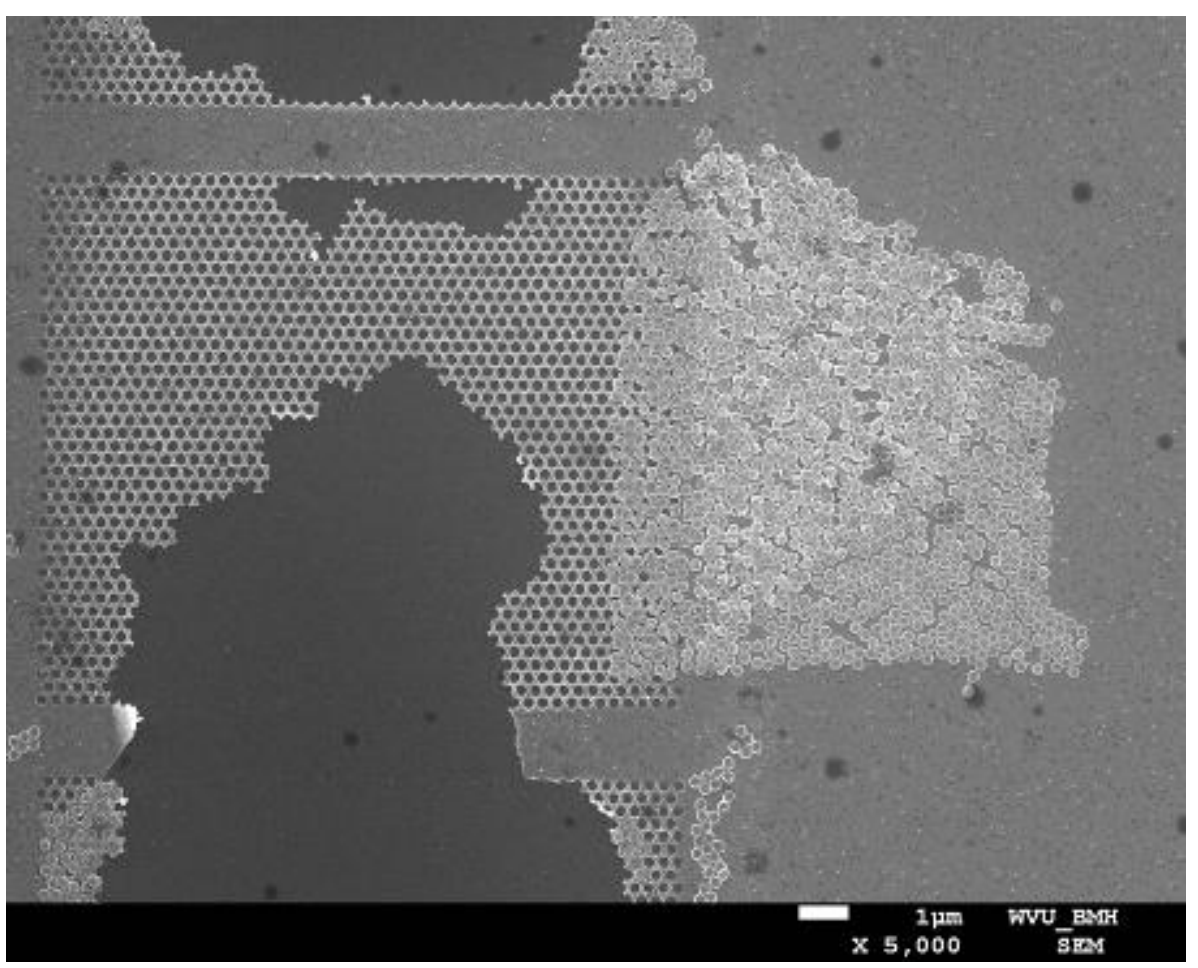

Figure 4.10. SEM image demonstrating an over sonication result. This will cause the thin $\mathrm{Ni}$ layers to break and not remain attached to the Si to act as a hard etching mask. 


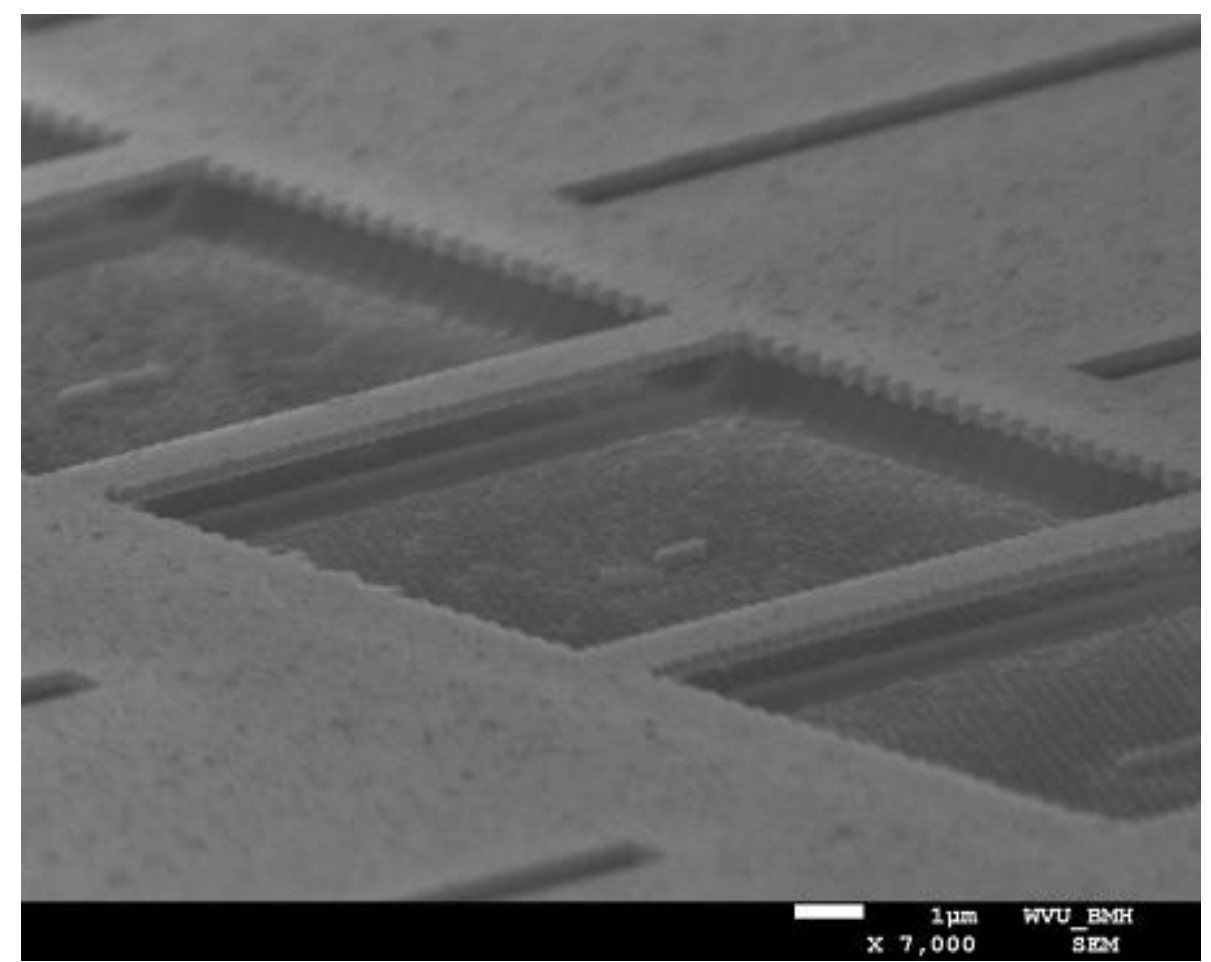

Figure 4.11. Collapsed PhC structures due to a drying step with a high Nitrogen flow

\subsubsection{Fabrication of Symmetric Photonic Crystal Lattices of Silicon Pillars and Air}

In the previous section, the fabrication procedure of a photonic crystal lattice of air holes in silicon was discussed. The vertical symmetry in that lattice was achieved once the $\mathrm{SiO}_{2}$ layer was wet etched in Hydrofluoric acid. In this section, the inverse of this process will be discussed. Specifically, the fabrication process to achieve a final vertically symmetric photonic crystal lattice of silicon pillars (rods) surrounded by air will be discussed. Of course, the vertical symmetry in air or any other liquid material of this structure is not possible. Therefore, the underlying $\mathrm{SiO}_{2}$ sacrificial layer will be kept in such a way the final structure can be sandwiched between it and a top deposited or placed oxide slab to achieve the desired vertical symmetry above and below the photonic crystal plane. Moreover, the fabricated $\mathrm{PhC}$ lattices of pillars can also be 'extruded' to the underlying $\mathrm{SiO}_{2}$ layer, making the effective refractive index below the slab even lower than that of $\mathrm{SiO}_{2}$ alone and therefore an almost symmetric structure can be resolved when toluene or any other liquid fills the region above, within, and bellow the pillars.

This process involves using a positive tone e-beam resist. The mechanism of exposing and developing a pattern written in a positive resist is explained in Figure 4.12. PMMA (polymethyl methacrylate), MicroChem, was the selected resist for this process. It is a versatile polymeric material that is very well suited to produce high resolution positive patterns when a direct write e-beam system is used. 
Specifically, PMMA 950K A3 is used throughout this work. The 950K means it has a 950,000 molecular weight (MW) which resins in an anisole solvent while the A3 indicates a specific commercial viscosity label that MicroChem uses to indicate that it less viscous than A4 and more viscous than A2.

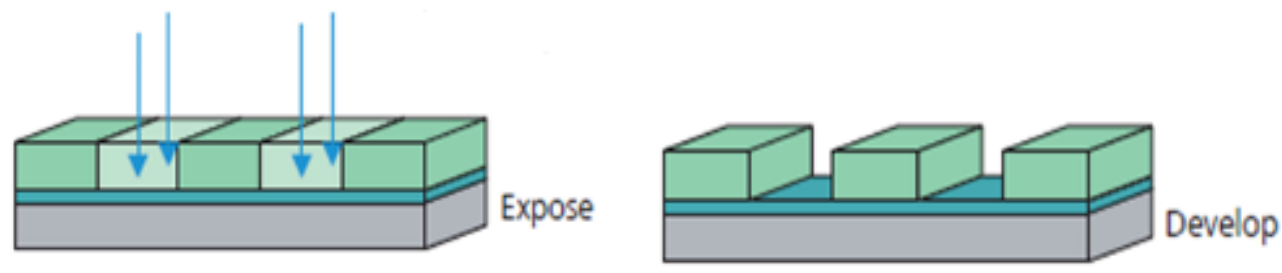

Figure 4.12. Positive-tone e-beam resist exposure and development mechanism [64]

The fabrication started with a regular degreasing step in which the SOI sample were sonicated for 5 minutes in Acetone and then immersed in Methanol for another 5 minutes. The sample was then rinsed with DI water for 5 minutes, dried with a steady Nitrogen gas flow produced by a Nitrogen gun, and then put in an oven set to $120^{\circ} \mathrm{C}$ for 20 minutes for a dehydration step. At this time the sample surface was ready to be coated with the resist. In order to produce pillars of silicon, the PMMA 950K A3 was used as explained above. This was simply due to later lift-off process that would be carried out to allow Ni to act as a hard mask when transferring the final structure to the underlying Si layer.

The PMMA A3 was the spun on the wafer for 50 seconds at $5000 \mathrm{rpm}$. This was followed by a soft bake step to remove solvents and make the resist more sensitive to the electron beam. The baking step was done on a hot plate set to $180^{\circ} \mathrm{C}$ for 90 seconds to achieve a final thickness of approximately 110 $\mathrm{nm}$. The EBL process was then done using a JEOL 7600 SEM system with e-beam lithography capability.

The pattern created in DesignCAD with submicron lattice dimensions was loaded to the NPGS software and converted into a run file that controlled the deflectors and the beam blanker by sending electric signals to the SEM tool. A detailed procedure of creating DesignCAD patterns and operating the e-beam lithography instrument is explained in Appendices A and B, respectively. Once the beam was focused, the writing of the pattern was done at a dose range between $320-335 \mu \mathrm{C} / \mathrm{cm}^{2}$. This dose range was found to be optimal to produce the best patterns on SOI samples. The write procedure was then followed by an immediate development step in an MIBK:IPA developer (1:3) for 65 seconds. To prevent overdevelopment from happening, the sample was then immediately immersed in Isopropyl Alcohol for at least 30 seconds and then rinsed with DI water for another 30 seconds before the sample was dried with a steady flow of $\mathrm{N}_{2}$ gas produced by a Nitrogen gun.

At this stage, the sample contained a pattern that can be observed in an optical microscope. Submicron features, of course, were not visible. However, an idea of how well the development/exposure 
processes could be examined. The pattern was a two dimensional photonic crystal lattice of holes in PMMA, which was the inverse of the final desired PhC lattice. Therefore, a thin layer of Ni was smoothly deposited using a Temescal BJD 2000 E-beam Evaporator to a thickness of approximately $20 \mathrm{~nm}$. Nickel was selected due to the high $\mathrm{Ni} / \mathrm{Si}$ etching selectivity which is approximately $1: 15$ at a $150 \mathrm{~W}$ RIE power [40]. Therefore, very thin layers of $\mathrm{Ni}$ are capable of withstanding a prolonged dry etch step and achieve depths of several hundreds of nanometers in Silicon.

The sample was then immersed in Remover PG (MicroChem ${ }^{\circledR}$ ) set to $65^{\circ} \mathrm{C}$ for 15 minutes followed by a two-second sonication step to ensure complete lift off. The Remover PG removed the PMMA polymer layer and therefore lifted off the Ni leaving behind only thin Ni pillars. The etching was done with a $\mathrm{CF}_{4} / \mathrm{O}_{2}$ gas mixture plasma using a Minilock-Phantom III ICP-RIE system. The chamber was first cleaned with a regular $\mathrm{O}_{2}$ plasma recipe for 10 minutes. The etching parameters were: $400 \mathrm{~W}$ ICP power, $150 \mathrm{~W}$ RIE power, $10 \mathrm{mTorr}$ chamber pressure, $27 / 3 \mathrm{sccm}-\mathrm{CF}_{4} / \mathrm{O}_{2}$ gas mixture flow rate ratio, DC bias $\sim-130 \mathrm{~V} .90$ seconds of etching using these previous parameters was sufficient to create $\sim 450 \mathrm{~nm}$ pillars in the top $\mathrm{Si}$ layer and the underlying $\mathrm{SiO}_{2}$ layer. The left Ni layer after the etching was then removed by dipping the sample in a Ni-etchant for 30 seconds with minor agitation. The sample was then finally cleaned in DI water and dried.

Once again, the symmetry could not be achieved by wet etching the sacrificial oxide layer beneath the silicon pillars as this will lift off the pillars and cause the whole structure to collapse. Therefore, especially for those triangular lattices of silicon pillars surrounded by air with wide directional bandgaps between the $\mathrm{M}$ and $\mathrm{K}$ Brillouin zone edges, the symmetry was achieved by either leaving the underlying $\mathrm{SiO}_{2}$ un-etched or extending the $\mathrm{PhC}$ lattice to the $\mathrm{SiO}_{2}$ layer to lower the effective refractive index below the PhC slab. Figure 4.13 demonstrates the process flow of this method while Figure 4.14 demonstrate an SEM image of the final fabricate features. The deviation between the as designed dimensions compared to the observed fabricated dimensions is summarized in Table 4.2.

In the previous section, the negative effects of several inaccuracies when following the discussed procedures were demonstrated. These included: resist under development, improper lift off by over sonication, and using a strong $\mathrm{N}_{2}$ flow when carrying out the drying steps. One other effect that must be discussed is that of an over etching step. The procedures discussed here utilize very thin Ni mask layers to ensure etch depths of several hundreds of nanometers in the underlying substrate, which is mostly $\mathrm{Si}$ for this work. However, sidewall anisotropy and smoothness are very critical parameters to be considered when prolonged etching procedures are desired to achieve high depths. These two parameters are severely affected when the Ni mask is completely used during the etching step. Therefore, it is advised that masks with thicknesses ranging between $20-30 \mathrm{~nm}$, should not be used to etch $\mathrm{Si}$ using a $\mathrm{CF}_{4} / \mathrm{O}_{2}$ gas mixture for periods longer than 3 minutes. The effect of over etching is demonstrated in Figure 4.15. 
Table 4. 2. The as-designed vs. as fabricated dimensions of the $\mathrm{PhC}$ in silicon. A deviation of only $0.87 \%$ was observed

\begin{tabular}{|c|c|c|c|c|c|}
\hline \multicolumn{6}{|c|}{ Triangular Lattice of Silicon Pillars $(r / a=0.33)$} \\
\hline \multicolumn{3}{|c|}{ As Designed Dimensions } & \multicolumn{3}{|c|}{ As Fabricated Dimensions } \\
\hline$d$ & a & $\mathrm{h}$ & $d$ & a & $\mathrm{H}$ \\
\hline 300 & 450 & $260 \mathrm{~nm}$ & 297.4 & 450 & $260 \mathrm{~nm}$ \\
\hline
\end{tabular}

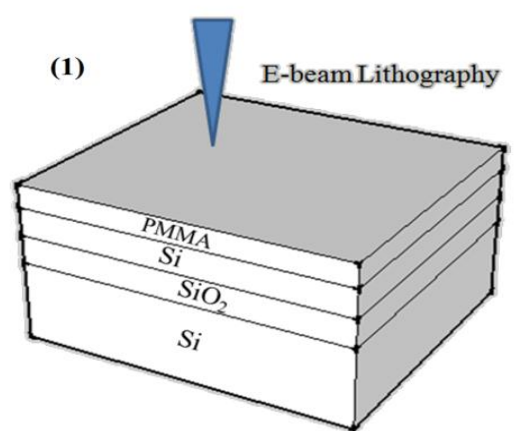

(3)

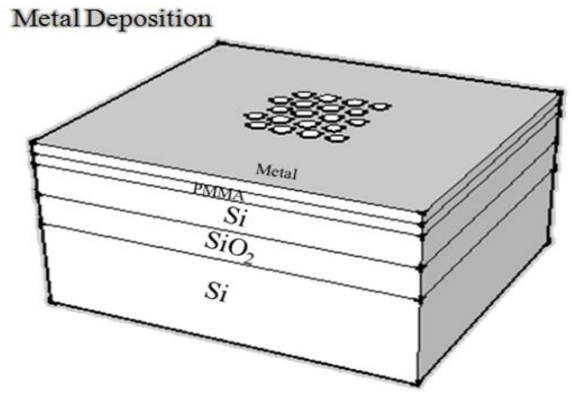

(2) Develop PMMA

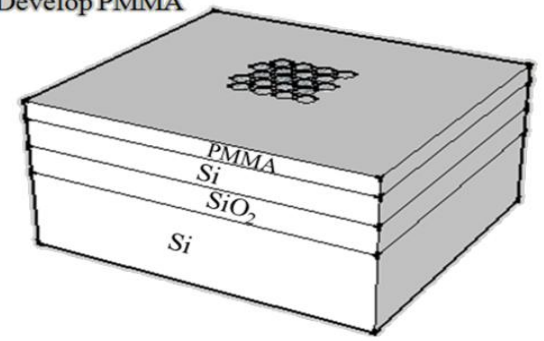

(4)

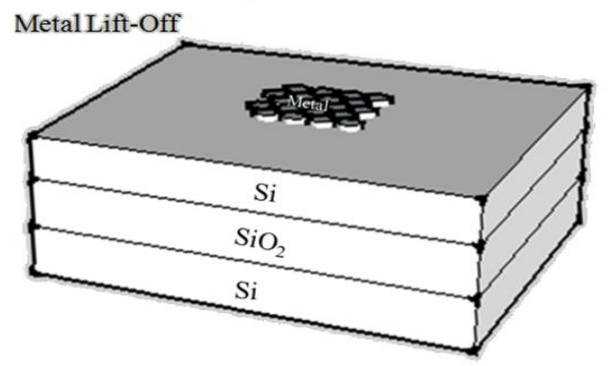

(5) Dry Etch

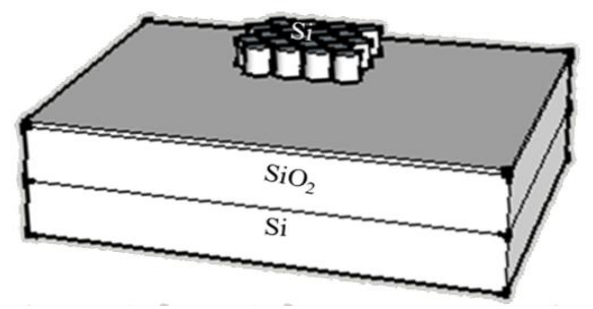

Figure 4.13. . Process flow of a symmetric PhC of pillars in air. (1) electron beam lithography in the PMMA resist. (2) development of the resist in 1:3 (MIBK:IPA) for 65 seconds. (3) E-beam Evaporation of a thin $\mathrm{Ni}$ layer ( $20 \mathrm{~nm})$. (4) Metal lift off in Remover PG for 15 minutes followed by a two second sonication (5) Dry etch of the silicon using a $\mathrm{CF}_{4} / \mathrm{O}_{2} 27 / 3$ gas mixture at $-85 \mathrm{~V}$ DC bias followed by a Ni removal step in $\mathrm{Ni}$

etchand. 


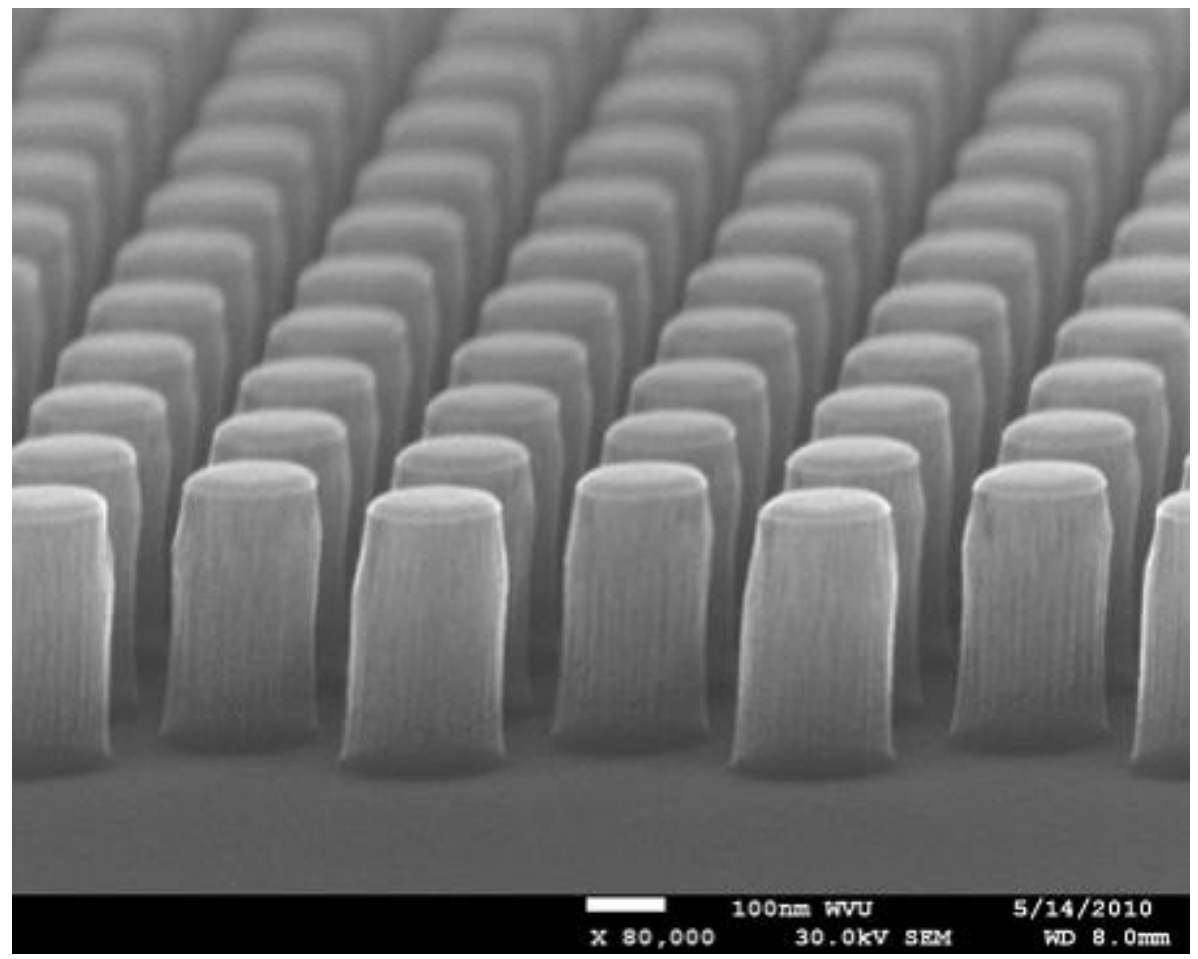

Figure 4.14. A tilted SEM image of the final structure of Silicon Pillars surrounded by air

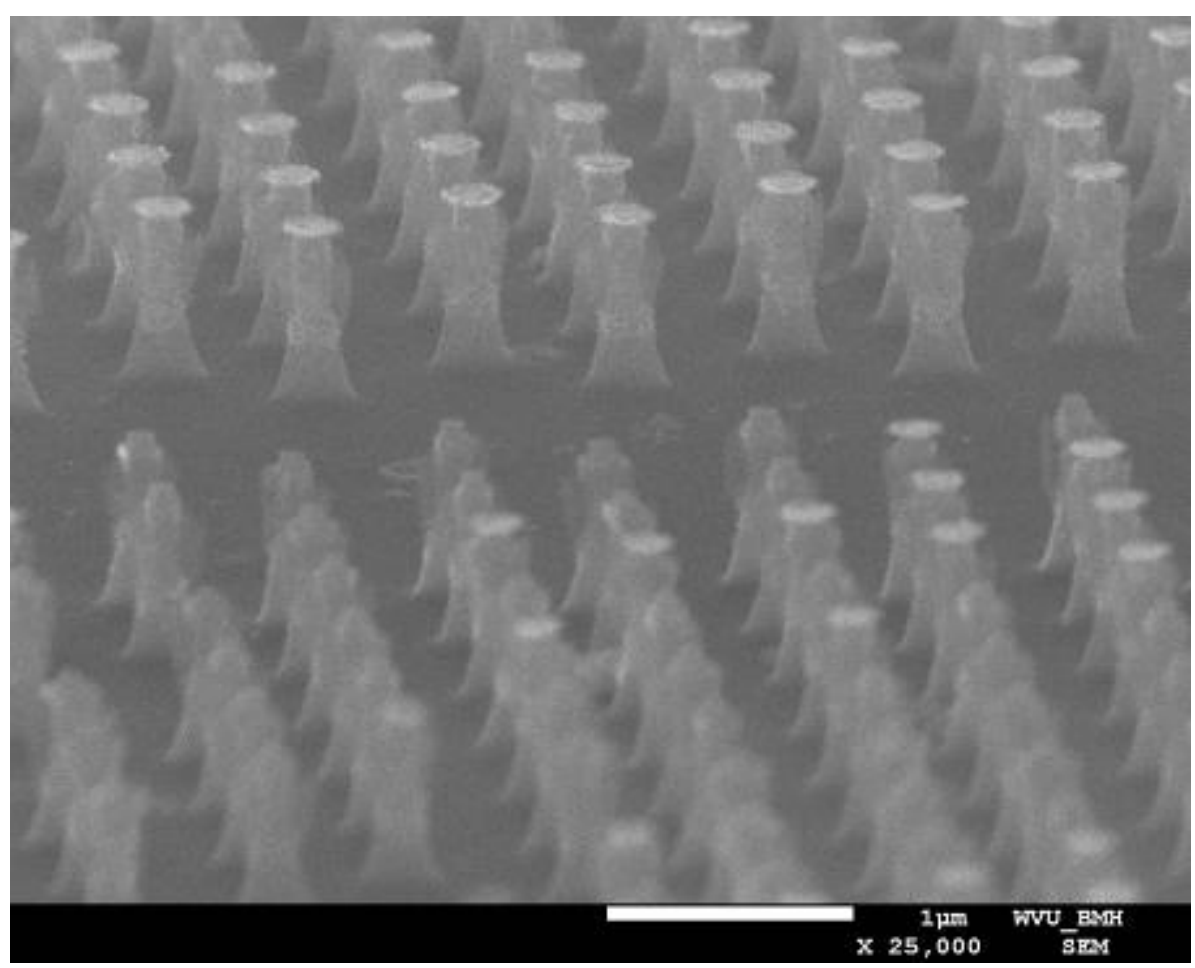

Figure 4.15. SEM of an over-etched $\mathrm{Si}$ sample in a $\mathrm{CF}_{4} / \mathrm{O}_{2}(27 / 3)$ gas mixture for 4 minutes 


\subsubsection{Hydrogen-Silsesquioxane (HSQ) Fabrication Recipe}

In the previous two sections, the fabrication process of holes in silicon and silicon rods surrounded by air was explained. It is obvious that these two processes involve multiple steps and the use of several microfabrication-based instrumentation in order to achieve final $\mathrm{PhC}$ structures in silicon. The main reason for this complexity is the resist poor dry etching resistance which fails to achieve desired depths in the underlying substrates when used directly as etching masks. Therefore, multiple steps of metal deposition and lift off are normally followed to create a hard mask that is more resilient to the dry plasma etching.

The previous fabrication complexities can possibly be resolved when using a HydrogenSilsesquioxane (HSQ) negative-tone electron-beam resist. It is currently commercially available from Dow Corning ${ }^{\circledR}$, and can be purchased in a wide range of concentrations when diluted in methyl-iso butyl ketone (MIBK). It has been widely used as a spin-on dielectric interlayer in the fabrication of integrated circuits. It is more desired than $\mathrm{SiO}_{2}$ due to its lower refractive index which reduces the resistancecapacitance (RC) time constant in the circuitry [65]. An HSQ molecule has a cage-like structure made of Hydrogen, Silicon, and Oxygen (Figure 4.16).

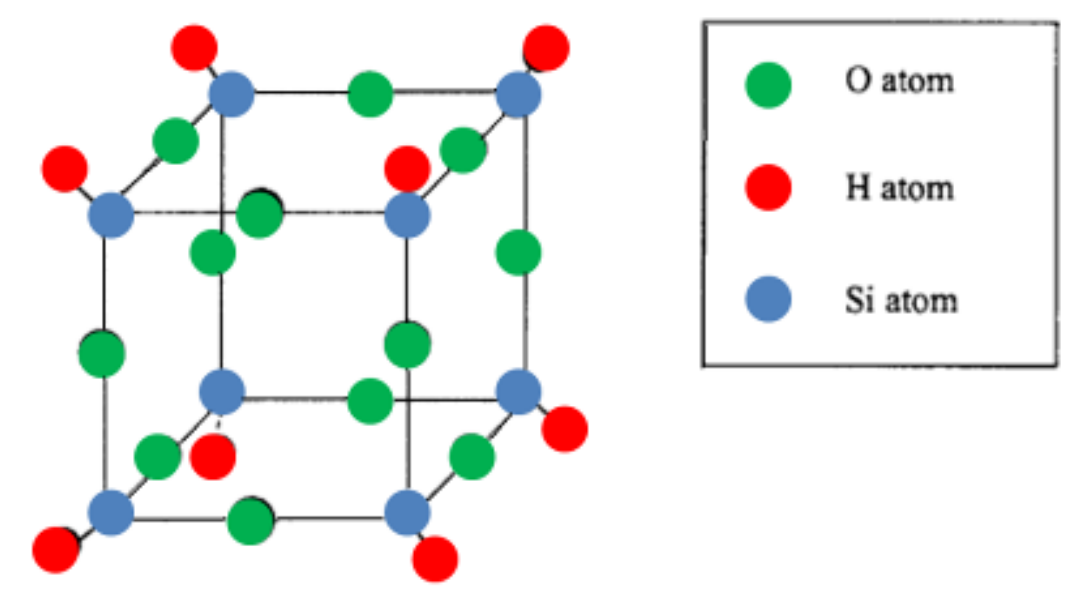

Figure 4.16. The cage-like structure of an HSQ molecule [65]

When exposed to an electron beam during a regular e-beam lithography procedure, the highly energetic electrons acquire sufficient activation energy capable of breaking the weak $\mathrm{Si}-\mathrm{H}$ bonds and promote the formation of the stronger siloxane (Si-O-Si) bonds [65-70]. Therefore, electron beam irradiation causes resist cross linking and the cage-like structures to become a long-range-network structure [71]. 
The most interesting aspect of HSQ is the ability to convert the top surface of a developed HSQ into a predominantly $\mathrm{SiO}_{2}$ layer that is hydrophilic and can become an instant hard-etching mask to the underlying substrate, i.e. Silicon or GaN. Throughout this section, the spinning, exposure, development, and etching recipes of HSQ (FOx-14), Dow Corning, is discussed.

In order to realize the optimal electron beam exposure recipe, first the spin and thickness relationship must be observed. Therefore, 6 silicon samples were degreased by a sonication step for 5 minutes in Acetone followed by Methanol for another 5 minutes. The sample were then rinsed with DI water, dried with nitrogen and then put in an oven set to $120^{\circ} \mathrm{C}$ for 20 minutes for a dehydration step. At this time the sample surface were ready to be coated with the resist. HSQ has a tendency to coagulate [65], therefore it should always be kept in its designated plastic bottles and refrigerated. Before spinning it, the bottle must be taken out of the refrigerator to warm up to room temperature $(\sim 25 \mathrm{C})$.

The six samples were spun at different speeds that were varied between 1000-6000 rpm in increments of $1000 \mathrm{rpm}$. Each sample was then baked on a hot plate set to $95^{\circ} \mathrm{C}$ for 4 minutes and then cleaved from the center where the resist is mostly uniform to accurately observe the thickness when crosssection sample imaging done using a JEOL 7600 SEM. Figure 4.17 demonstrates a plot of the average thickness that was recorded for each spinning speed.

The thinnest HSQ sample (spin speed $6000 \mathrm{rpm}$ ) was used toward the end of this optimization procedure. This was mainly due to the limitation of the electron beam energy of the available e-beam lithography system as the maximum beam energy of $30 \mathrm{kV}$ can be achieved using this tool and thicker HSQ layers require energies higher than $30 \mathrm{kV}$. For $6000 \mathrm{rpm}$ spin speed, the HSQ thickness was around $267 \mathrm{~nm}$. At the beginning, and in order to develop an exposure recipe for this thickness, a DesignCAD file was generated to produce a triangular photonic crystal lattice of HSQ rods upon development. In this file, the dose was first varied from $100 \mu \mathrm{C} / \mathrm{cm}^{2}$ to $600 \mu \mathrm{C} / \mathrm{cm}^{2}$. The reason for this wide range was mainly due to the fact that an accurate approximate dose was not yet known. Figure 4.18 demonstrates SEM images of the different doses. The optimal dose was found to be in the range between 285 and 315 $\mu \mathrm{C} / \mathrm{cm}^{2}$. This range produced the best features with dimensions very close to the designed dimensions. For this sample, a development time of 11 minutes in MF CD-26 (Shipley) was used based on ref. [65] in which a diluted FOx-14 HSQ resist was used and an 8-mintute developed was done. 


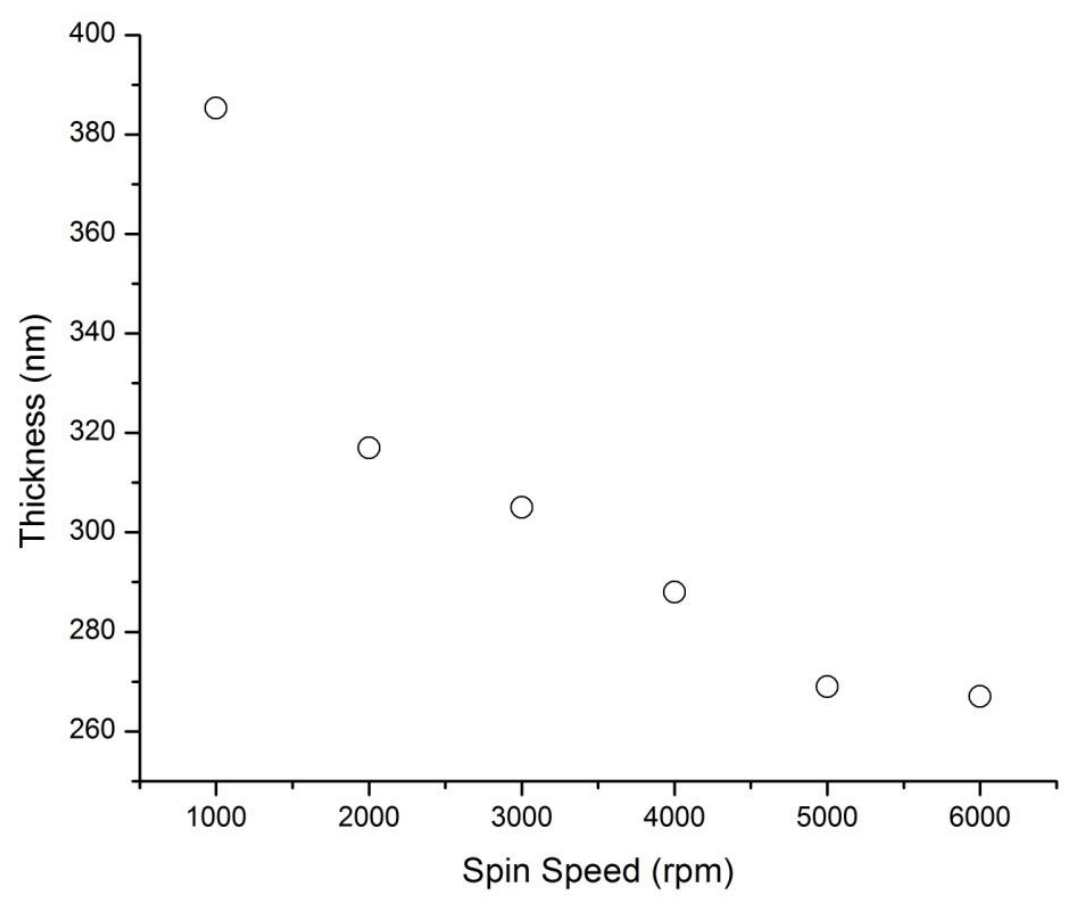

Figure 4.17. Spin speed vs. thickness in nanometer for HSQ (FOx-14)

In order to reach a decision of whether the range between $285-315 \mu \mathrm{C} / \mathrm{cm}^{2}$ is indeed the best dose range for FOx-14 of approximately $235 \mathrm{~nm}$ thickness, a development optimization process was done. In this process, the pattern dosage range was varied between $270-360 \mathrm{uC} / \mathrm{cm}^{2}$ and 4 samples were written using e-beam lithography and developed in MF CD-26. Four development times were studied for this experiment: 5 minutes, 8 minutes, 12 minutes, and 18 minutes. These different development times were selected based on the initial results that were observed. For example, in Figure 4.18, it is clear that 11 minutes of development was sufficient to observe an HSQ patterns for the whole dose range from 100 to $600 \mu \mathrm{C} / \mathrm{cm}^{2}$. However, at doses lower than the desired range $\left(285-315 \mu \mathrm{C} / \mathrm{cm}^{2}\right)$, the HSQ pillars were 30 $\mathrm{nm}$ smaller than the actual diameter. On the other hand, doses higher than the desired range demonstrated regions of undeveloped HSQ between the pillars which indicates that the beam energy was high and caused proximity exposure effect. The desired range showed patterns within tolerable shifts from the desired dimensions as well as almost complete development of the HSQ pillar. The results of the different development times are summarized in the SEM images of Figure 4.19. 

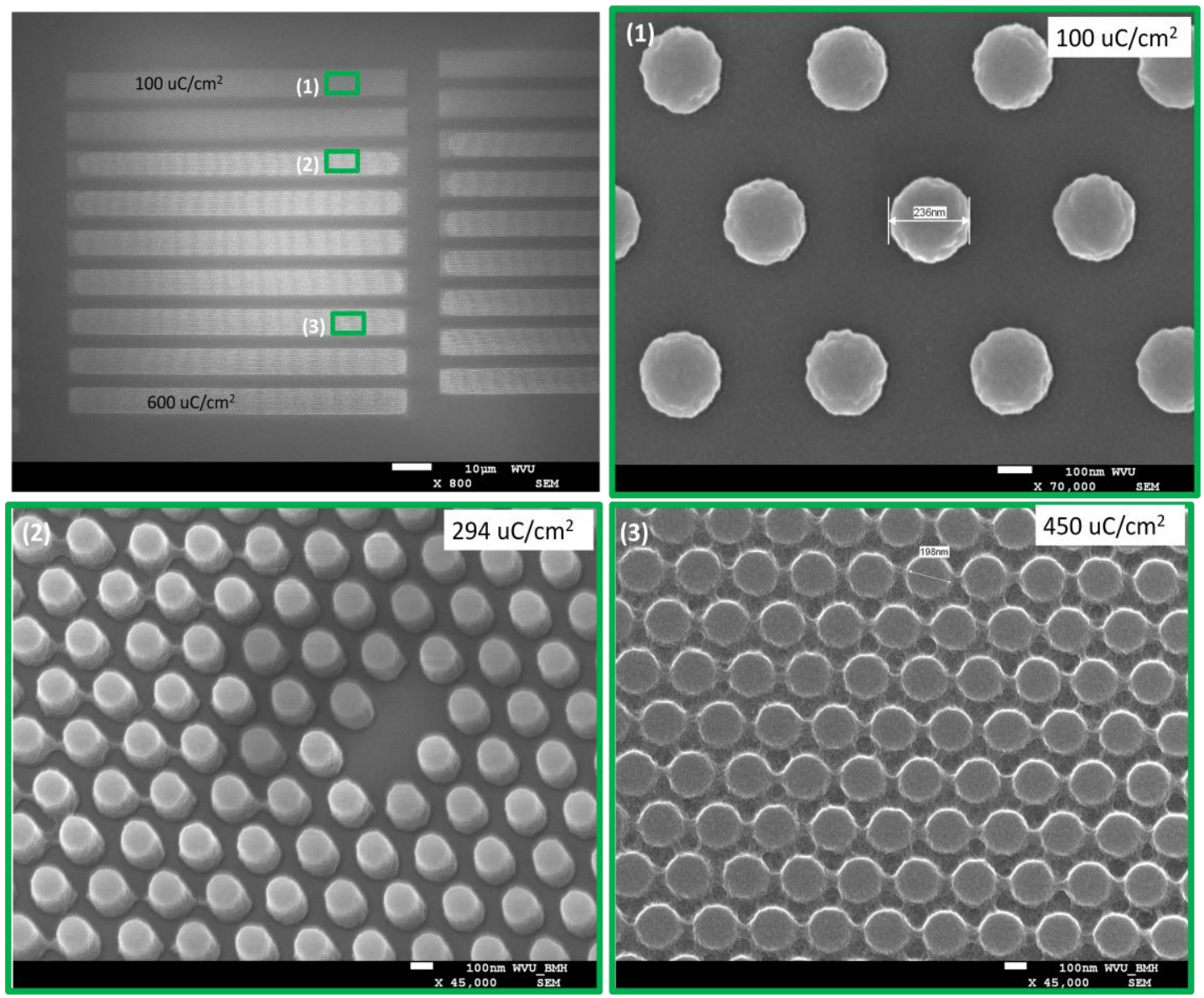

Figure 4.18. SEM images demonstrating the exposure-recipe optimization patterns. Dose range is from $100-600 \mathrm{uC} / \mathrm{cm}^{2}$ and development time is 11 minutes. Zoomed-in SEM images of the boxed regions in the top left SEM image are shown in the numbered images 1,2, and 3.

It is obvious from Figures 4.19 and 4.20 that development time of 5 minutes and 8 minutes were not sufficient to fully resolve the exposed HSQ pillars. On the other hand, a development time of 18 minutes caused a serious distortion in the shape of the HSQ pillars within the desired range. Twelve-minute development resolved the best anisotropy of features' side walls as well as the best desired dimensions (diameter $260 \mathrm{~nm}$ ).

However, the main objective of this work is to demonstrate the ability to use HSQ as a hard etching mask when etching semiconductor materials such as: GaN and Silicon. The main objective is to transfer the top layer of the HSQ pattern into an oxide layer that can withstand prolonged dry etching processes. This is normally done by oxygen plasma treatment step after the development of the HSQ and before the etching step. The HSQ $\mathrm{O}_{2}$ treatment was done in a March PX-250 Plasma Asher. The plasma parameters were as follows: $300 \mathrm{~W}$ power, $300 \mathrm{mTorr}$ pressure, $38 \mathrm{O}_{2}$ gas set point, and 600 seconds. After the 10minute plasma treatment step, the Si sample was then etched using two $\mathrm{CF}_{4} / \mathrm{O}_{2}$ gas mixtures for 60 seconds. Figure 4.21 demonstrates SEM images of these two etching results. 

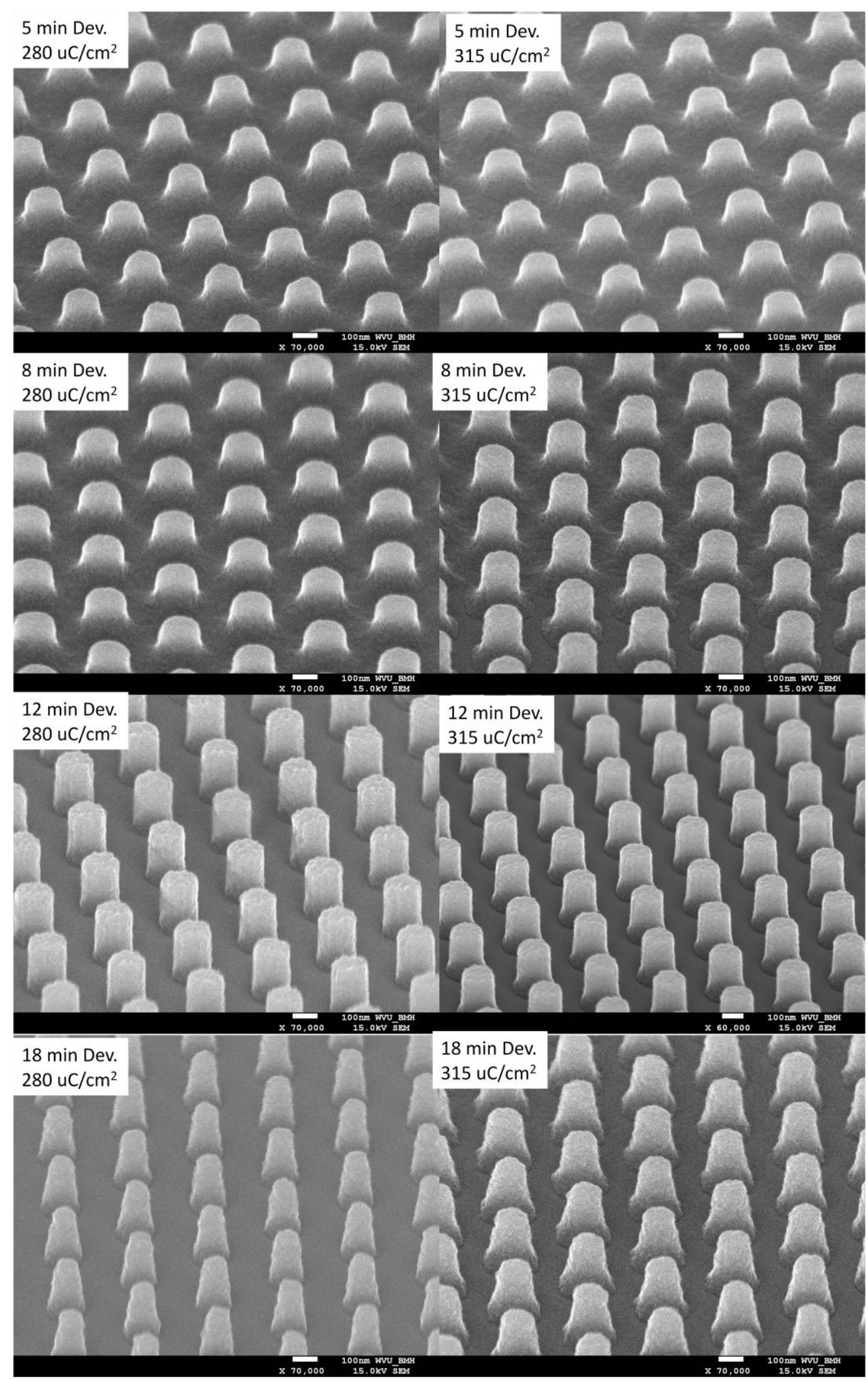

Figure 4.19. SEM images of the desired range of doses over different development times; 5, 8,

12 , and 18 minutes $\left(1^{\text {st }}, 2^{\text {nd }}, 3^{\text {rd }}\right.$, and $4^{\text {th }}$ row respectively $)$ 


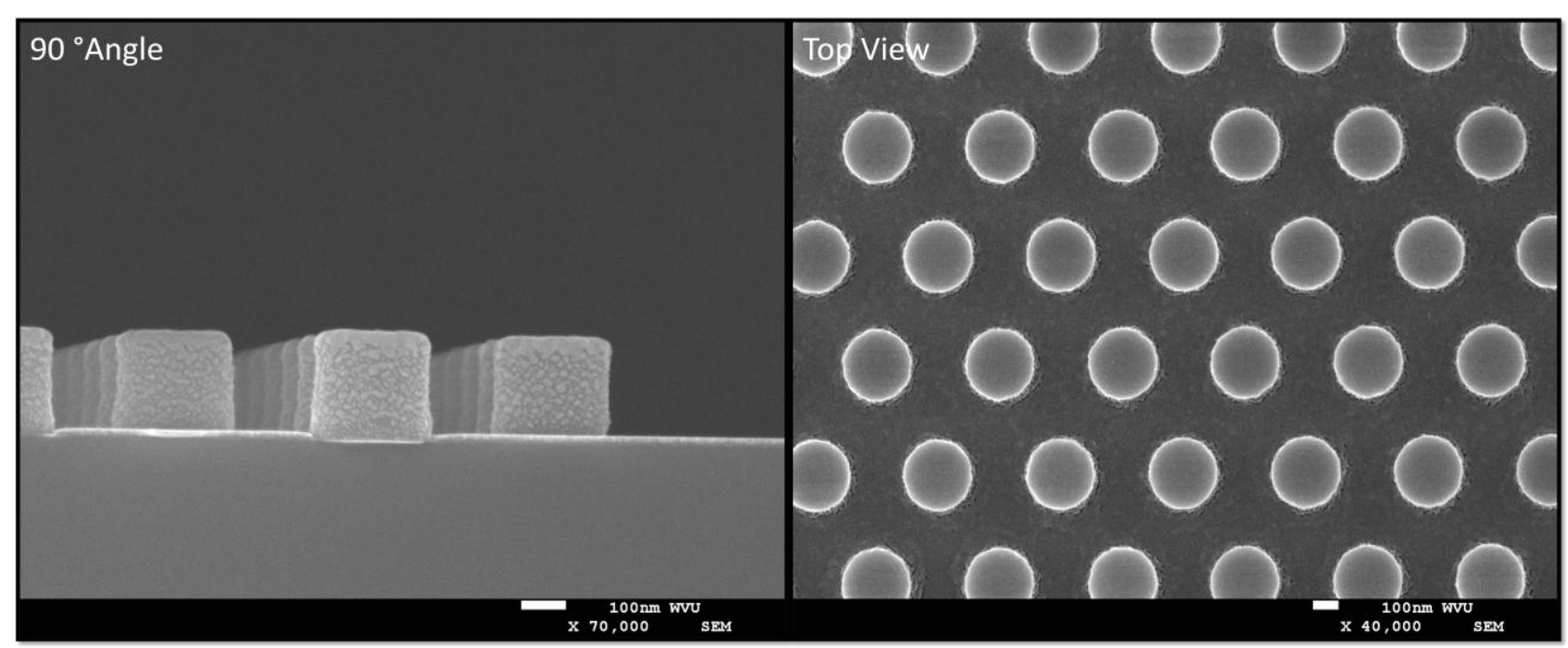

Figure 4.20. SEM images showing (left) a $90^{\circ}$ cross-section image of a $315 \mu \mathrm{C} / \mathrm{cm}^{2}$ dose of HSQ on silicon and 12-minute development. (Right) A top view of the pattern.

For both etching recipes, the following parameters were kept constant: $400 \mathrm{~W}$ ICP power, $150 \mathrm{~W}$ RIE power, and $10 \mathrm{mTorr}$ chamber pressure. However, for the first sample whose results are demonstrated in the top row of Figure 4.21, a $27 / 3-\mathrm{CF}_{4} / \mathrm{O}_{2}$ gas mixture was used. After 1 minute of etching using this recipe, the Si pillar depth was measured to be approximately $164 \mathrm{~nm}$ while the HSQ was totally removed to achieve a selectivity of $1 / 0.7$ (HSQ/Si). The second etching recipe used a 18/2 $\mathrm{CF}_{4} / \mathrm{O}_{2}$ gas mixture. After 1 minute of etching, the HSQ mask was etched away but the Si pillar had a depth of approximately $218 \mathrm{~nm}$. The selectivity for this second recipe is $1 / 0.75$ (HSQ/Si).

The successful etching results demonstrated in Figure 4.21 prove the ability of HSQ to act as a silica-like mask when etching underlying substrates. However, the patterns shown in the figure are for these of silicon pillars surrounded by air. It will be very convenient to develop also exposure and development recipes of the inverse of that pattern in order to achieve final structures of holes in silicon. To accomplish this with HSQ's negative-tone behavior, a DesignCAD file must be designed with stitched features that will cause the beam to turn on and expose the surrounding region of the holes and not the holes themselves as was done previously. After development, the pattern in HSQ is expected to be a mesa-like structure of a two-dimensional photonic crystal of air holes in HSQ. The concept is clarified in Figure 4.22. The optimal dose to achieve the desired dimensions was found to be $\sim 290 \mu \mathrm{C} / \mathrm{cm}^{2}$ while the optimal development time was around 60 minutes. Figure 4.23 demonstrate SEM images of a fully resolved inverse pattern. A $\mathrm{CF}_{4} / \mathrm{O}_{2}$-plasma was used again to etch the features and produce the final structures in silicon. The remaining HSQ was removed by dipping the sample in HF followed by a rinse with DI water. Figure 4.23 demonstrate the mesa-like $\mathrm{PhC}$ structures in silicon. 


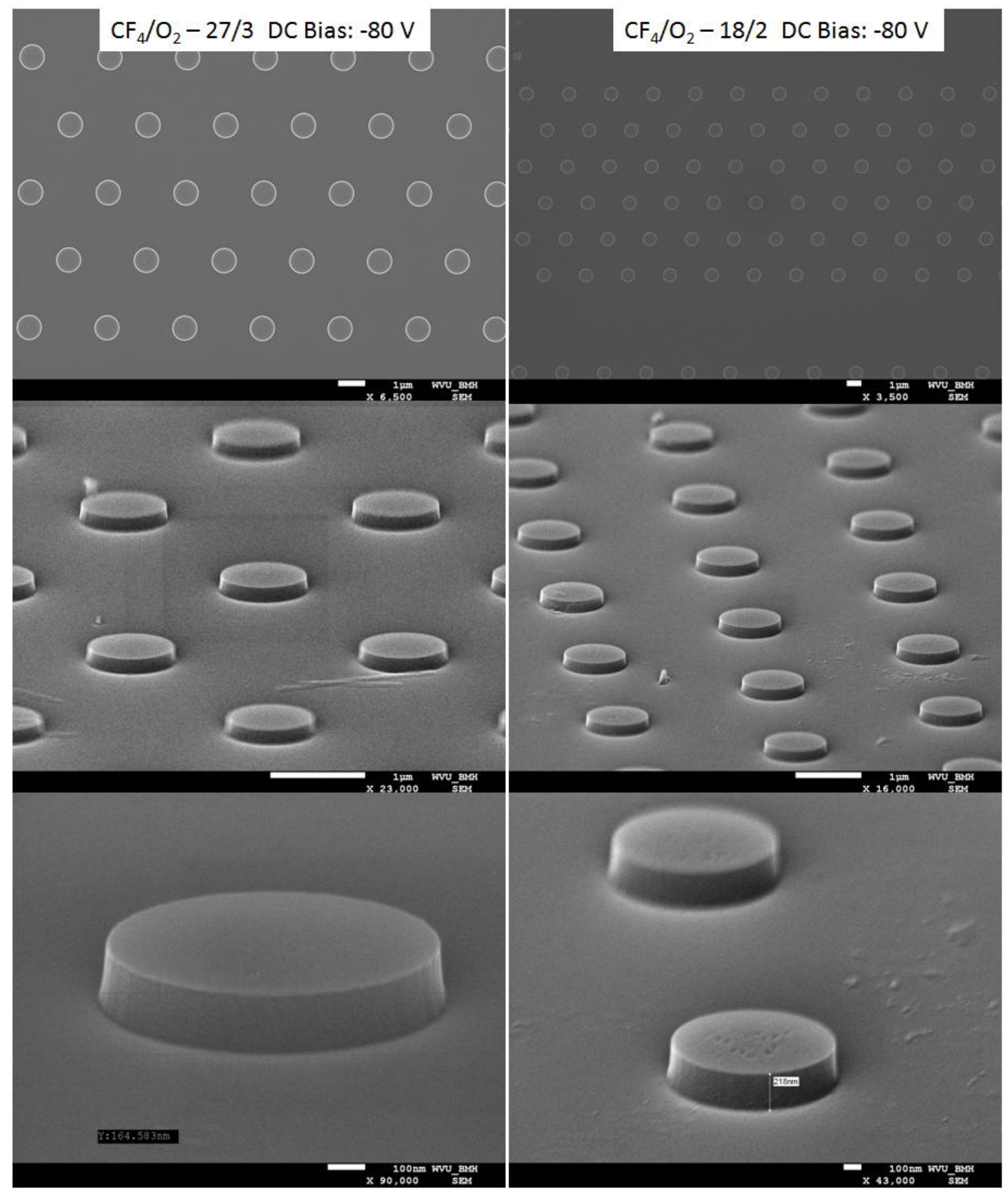

Figure 4.21. SEM images showing the final structure Si pillar etched using HSQ as a hard mask. Left column demonstrates images of etched silicon using a $27 / 3\left(\mathrm{CF}_{4} / \mathrm{O}_{2}\right)$ etch recipe while the right column shows SEM images of etched Si using a 18/2 $\left(\mathrm{CF}_{4} / \mathrm{O}_{2}\right)$ etch recipe 


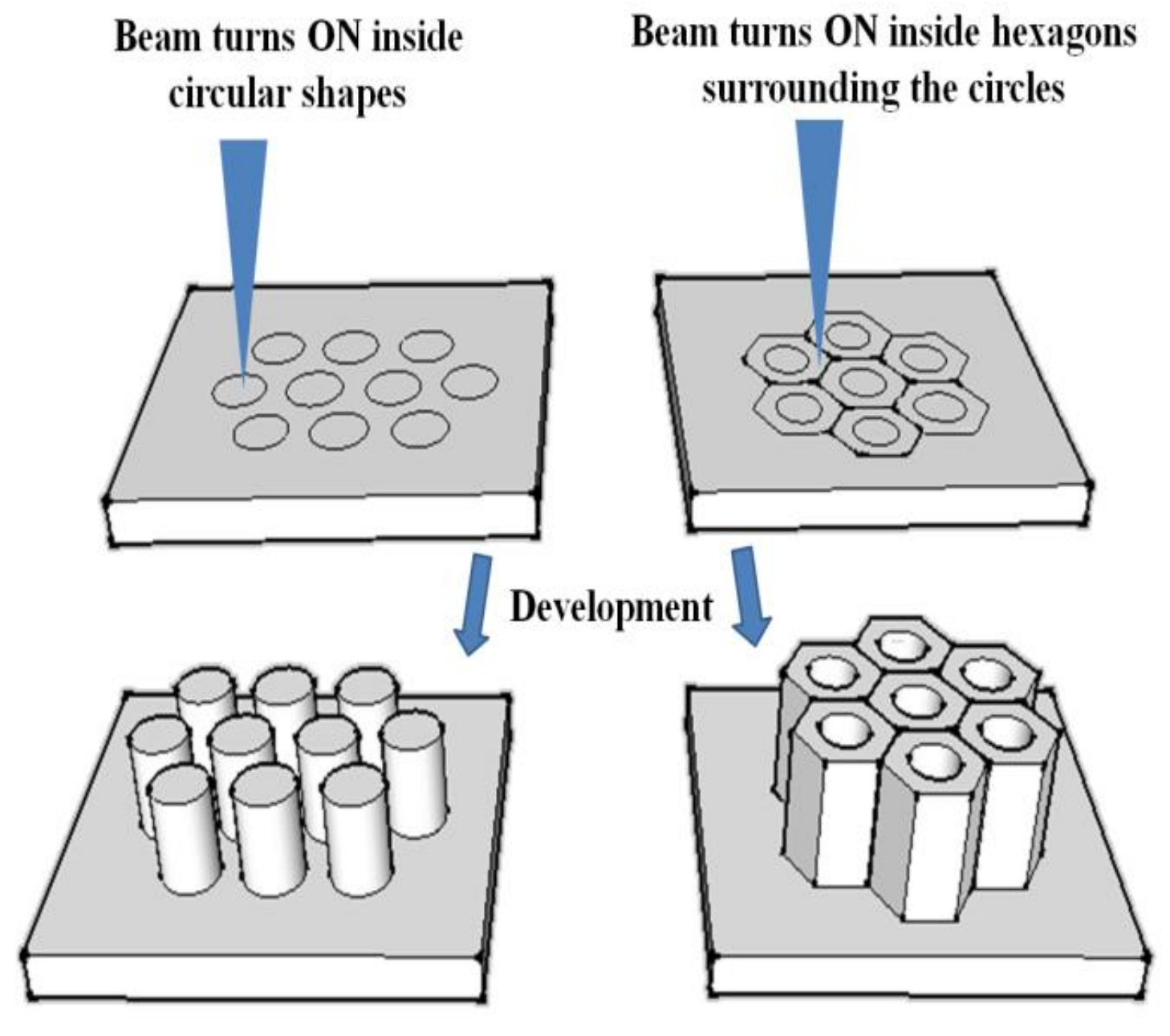

Figure 4.22.Inverse pattern writing technique using single unit stitching method when creating the DesignCAD file 

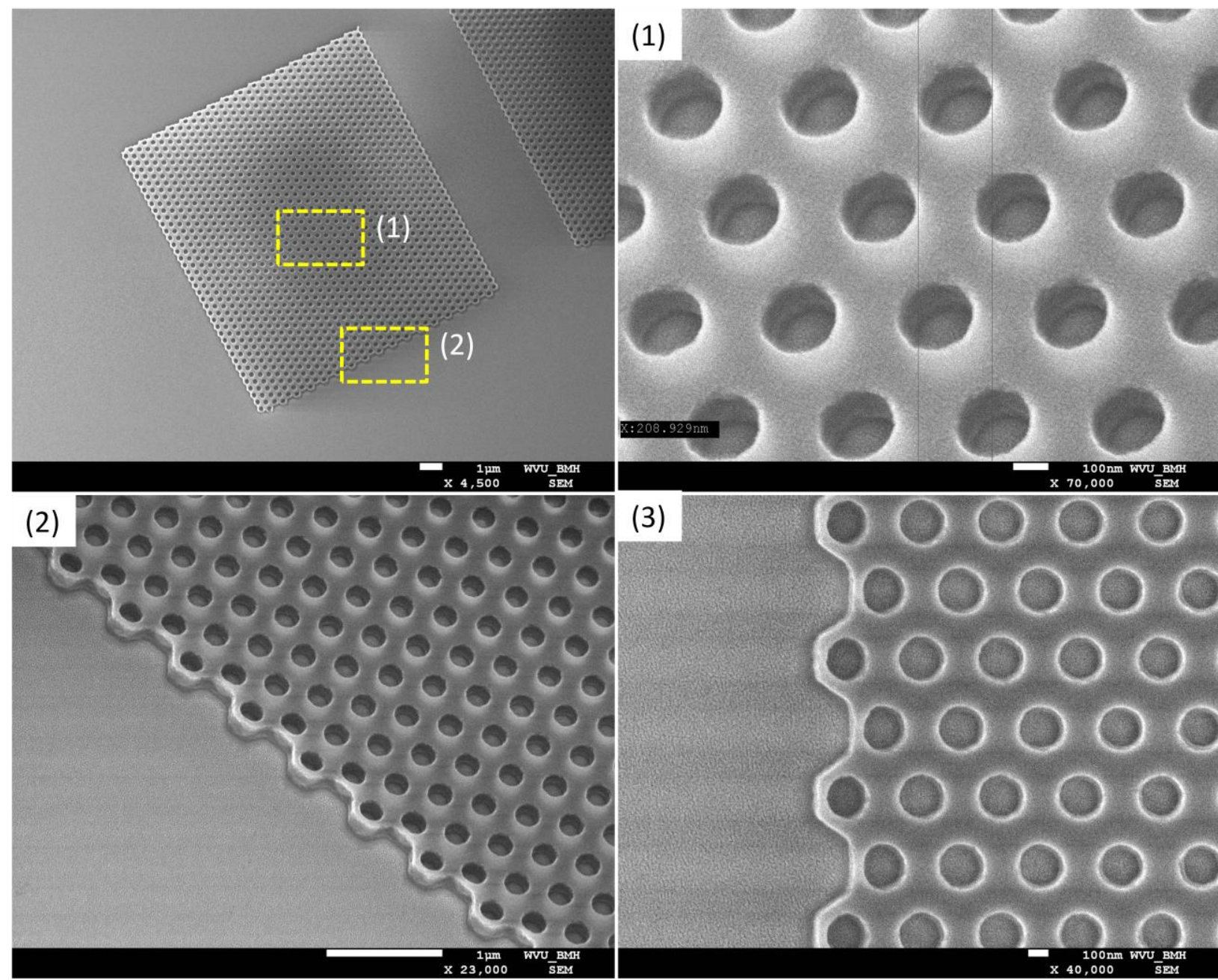

\section{(3)}

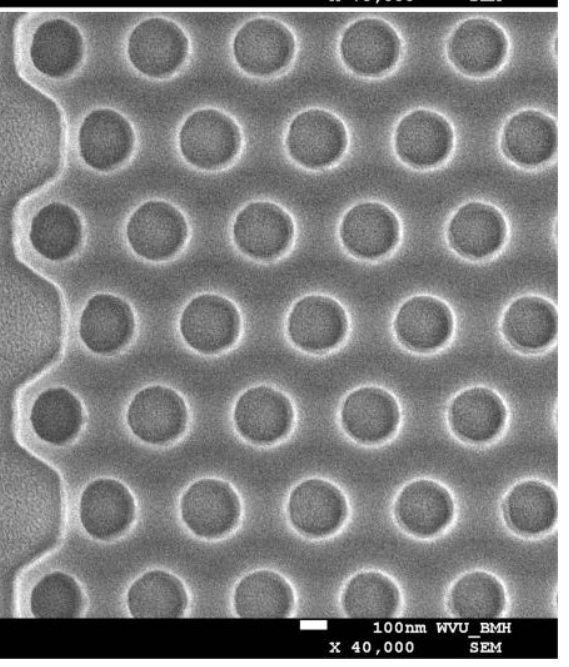

Figure 4.23. SEM images of the mesa-like PhC pattern in HSQ. Images (1) and (2) represent tilted are zoomed-in images of the dashed regions in the top left SEM image. (3) a top-view SEM image of the mesa edge. Scale bar is 100 $\mathrm{nm}$ in all images except for image (2) in which the scale bar is $1 \mu \mathrm{m}$

\section{(1)}

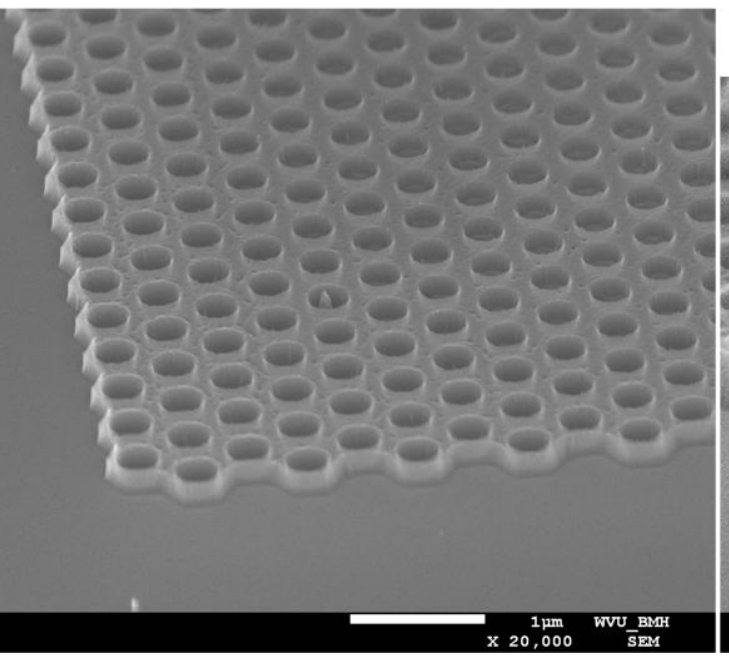

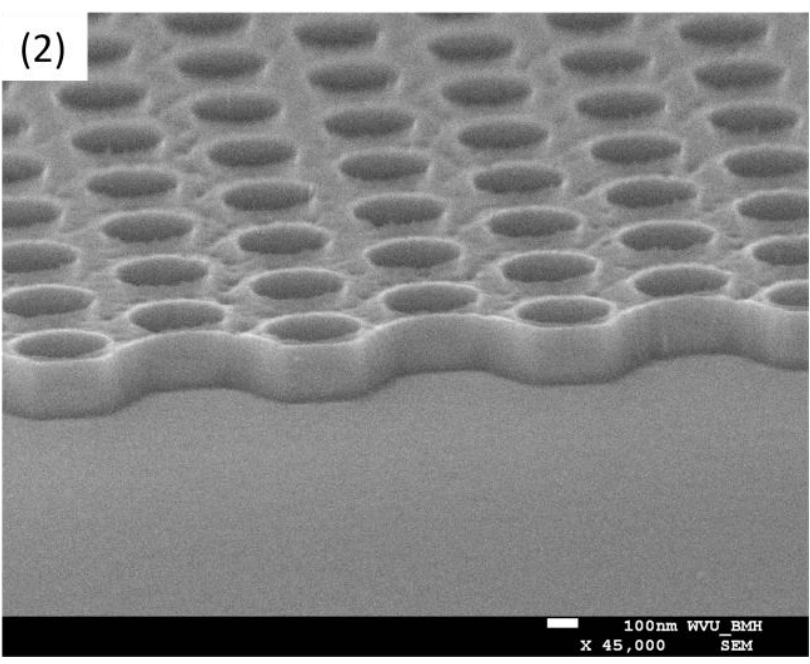

Figure 4.24. Tilted SEM images of the final fabricated PhC structures in silicon using HSQ as a mas. (1) $70^{\circ}$ angle 
In order to compare HSQ's performance as a negative tone electron beam resist as well as a hard etching mask to other ordinary polymer electron beam resists, ma-N 2403 (MicroResist Technology GmbH) and PMMA 950K A3 (MicroChem) were used. For ma-N experiment, the resist was spun at 5000 rpm for 50 seconds and baked on a hot plate set to $95^{\circ} \mathrm{C}$ for 3 minutes to achieve a thickness of approximately $240 \mathrm{~nm}$. The optimal electron-beam dose was approximately $63 \mu \mathrm{C} / \mathrm{cm}^{2}$ for this resist. The sample was then developed in ma-D 525 for 70 seconds before it was hard baked on a hot plate set to $100^{\circ} \mathrm{C}$ for 1 minute. The sample was then etched using the same etching parameters as explained above for the treated HSQ samples. After the etching step, the sample was dipped in Remover PG (MicroChem) set to $65^{\circ} \mathrm{C}$ for 15 minute, rinsed with DI water and then dried with a Nitrogen gun. The etched features were observed using a JEOL 7600 SEM. Figure 4.24 (1) demonstrates the final etched structures in silicon. This image demonstrates the poor etch selectivity of ma- $\mathrm{N}$ to silicon as the height of these features was measured to be approximately $140 \mathrm{~nm}(0.58$ etch selectivity). Moreover, the isotropic side walls demonstrate ma-N's failure to act as a hard etching mask when compared to the well defined pillars produced by HSQ in Figure 4.21.

As for the PMMA experiment, the sample was first cleaned and then spun with the PMMA resist at 5000 rpm for 50 seconds before it was soft baked on a hot plate for 90 seconds at $180^{\circ} \mathrm{C}$ to achieve a final thickness of approximately $80 \mathrm{~nm}$. A PhC pattern was then written using a JEOL $7600 \mathrm{EBL}$ tool at a $330 \mu \mathrm{C} / \mathrm{cm}^{2}$ area dose. The sample was then developed in a Methyl Isobutyl Ketone and Isopropyl Alcohol solution mixed in a 1:3 ratio (MIBK:IPA) followed by a 20 second rinse in IPA. The sample was then hard baked in on a hot plate at $100^{\circ} \mathrm{C}$ for 1 minute and then etched using the sample etching parameters discussed above. The final etched features in silicon were analyzed using a JEOL 7600 SEM tool and a final thickness of the etched features was measured to be approximately $25 \mathrm{~nm}$. Therefore, demonstrating a poor etch selectivity of about 0.31 using this resist. Fig. 8 demonstrates a tilted SEM image of the final fabricated PhC features of holes in silicon using PMMA as a resist.

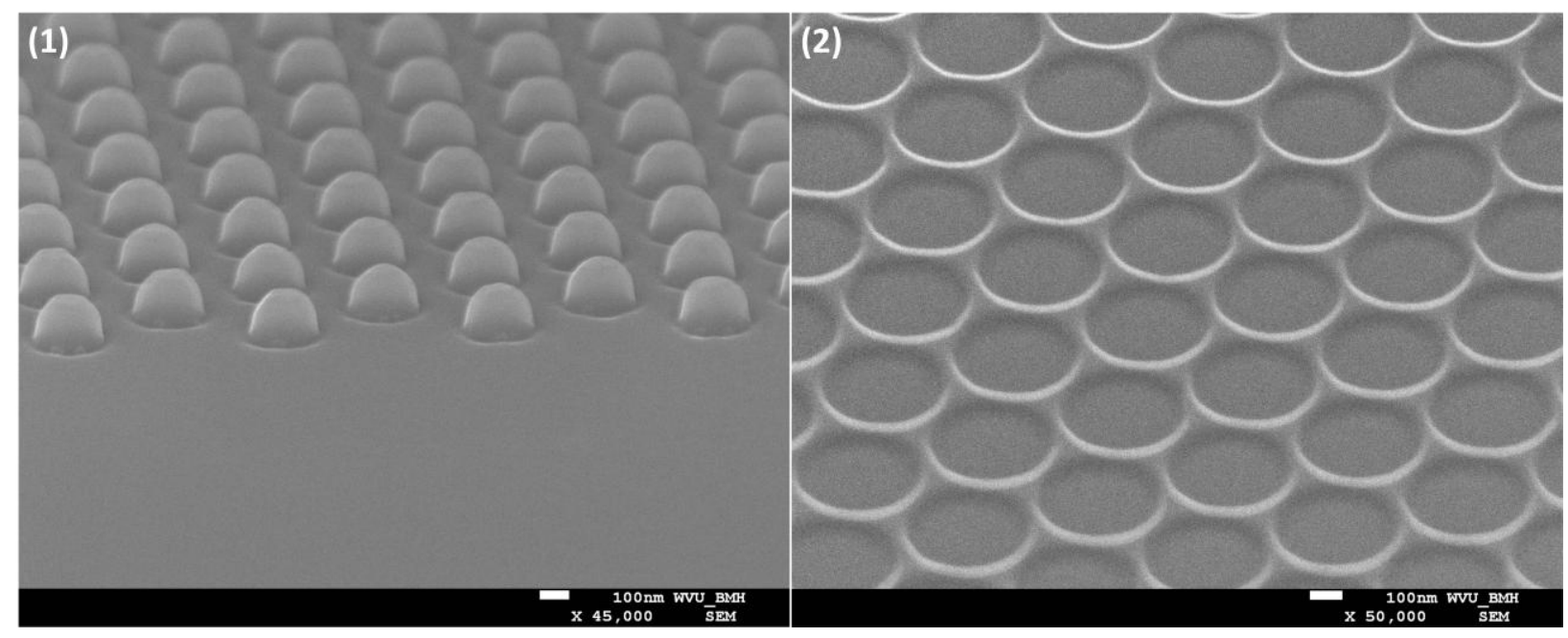

Figure 4.25. (1) Tilted SEM image of the Si pillars that were achieved when using maN as a hard etching mask. (2) The PhC structure in silicon using PMMA as a hard etching mask. 


\subsection{POLYMER-BASED PHOTONIC CRYSTALS}

Thin film semiconductor materials are commonly utilized as substrates in the fabrication of PhCs for sensing platforms due to the large refractive index contrast between these materials and the aqueous solution of the target analytes. This large refractive index contrast makes it easier to design a $\mathrm{PhC}$ that has a complete photonic bandgap. In the previous section, the fabrication processes to produce these nanoscale features were demonstrated. However, integrating small pieces of solid materials with polymerbased microfluidic structures is a complex procedure. Microfluidic structures in biosensors are primarily fabricated via soft-lithographic techniques in biocompatible materials, which are not readily amenable to the inclusion of sections of semiconductor wafer material containing suspended PhC films less than $500 \mathrm{~nm}$ thick. In fact, most polymers are considered relief structures to smooth semiconductor surfaces and that creates an issue if these semiconductor samples are expected to become stable core structures within a channel. It can be accomplished, but this integration step will significantly increase the final cost of system that utilizes this type of transducer, making them less attractive from a marketing standpoint [72-75].

$\mathrm{PhC}$ lattices fabricated in polymer materials can provide solutions to the previous limitations. Polymer materials are relatively cheaper than semiconductor materials and can be mass produced using the much simpler soft lithographic micro-scale-based techniques. In chapter 3, partial bandgaps were observed for PDMS and Epoxy for two dimensional triangular PhC lattices. The existence of a bandgap allows the 2D PDMS- and Epoxy-based PhC structures to be incorporated in biosensors utilizing either the active or passive modality. In the passive modality, the PDMS PhC slab can replace the Si slab and resonant cavities can be tuned for frequencies that fall within the narrow bandgaps to observe fluorescence enhancement. On the other hand, in the active modality, PDMS PhC structures can act as narrow transmission or reflection structures that can be tuned to display shifts when target analytes adsorb to the surface of a high RI material deposited on them [26] or to walls of the PhC pores [24].

Soft lithographic techniques, in general, and replica molding techniques, in specific, have successfully achieved sub-micron features in polymers only when the molded structures are about $1 \mu \mathrm{m}$ apart (low density features). Achieving this molding process in the nano-scale to allow for high density features to be molded from master mold samples repeatedly is the focus of this and the next two sections. Surface treatment and high vacuum are the two main critical steps to achieve this new scale of molding. 


\subsubsection{PDMS-Based Photonic Crystals}

Poly (dimethylsiloxane), or PDMS, is an elastomer that is considered a very common relief structure in soft lithography [72-75]. It has a unique combination of properties resulting from the presence of an inorganic siloxane backbone and organic methyl groups attached to silicon. This allows it to have a very low glass transition temperature and it is fluid in room temperature and can be readily converted into solid elastomers by cross-linking via heat [72].

PDMS is desirable for our application since the ultimate goal of this project is to produce a portable lab-on-a-chip rapid DNA detection system incorporating microfluidic channels which are mostly fabricated in PDMS. Therefore, if the integration step of these channels with $\mathrm{PhC}$ lattices can be made in a single step that does not involve several microfabrication processes or related instrumentation, then from a marketing stand point, this is very desirable. However, the replica molding still requires a master mold containing the negative pattern to start with. To achieve this, e-beam lithography was used to create only one master mold fabricated in silicon that could be used to produce several molded samples in PDMS.

To produce holes in PDMS, a silicon master containing pillars was first fabricated. The procedure to accomplish this first task is explained in section 4.5.2. Transferring the inverse pillars-in-air pattern into a $\mathrm{PhC}$ structure of air holes in PDMS was accomplished by first mixing PDMS and a curing agent in a 15:1 ratio, respectively. The mixture was then poured on top of the Si master-mold sample in a plastic Petri dish. To degas the PDMS and remove air bubbles and ultimately allow the relatively viscous PDMS to flow in-between the pillars, the Petri dish was put inside a desiccator connected to an XD-5 Edwards scroll pump to achieve high vacuum for 5 minutes $\left(\sim 10^{-2}\right.$ torr $)$. After 5 minutes, large bubbles can be clearly noticed on the top surface of the poured PDMS but a slow venting procedure of the desiccator quickly removed these bubbles. At this time, the sample was ready for curing and the Petri dish was transferred to an oven set to $60^{\circ} \mathrm{C}$ for 3 hours. At the end of the curing period, the Petri dish was taken out of the oven and left to cool down for 5 minutes before the PDMS was slowly peeled off the master mold to obtain the final PhC structure of air holes in PDMS. Figure 4.26 demonstrates this fabrication process while Figure 4.27 demonstrates SEM images of the master mold and the final molded structures. When the degassing step was done using house vacuum, the molded PDMS PhC structures were not fully resolved holes as can be seen in Figure 4.28. That demonstrates how critical the low pressure degassing step to the overall success of this method.

Examining the final PhC lattices in PDMS using SEM was a challenging task due to charging effects. Charging is considered a major hurdle in the generation of good SEM images when examining dielectric polymer materials. This effect is mainly due to the accumulation of excess electrons on the 
surface of the sample. In a conductive or semiconductor sample, the electron beam normally goes into the sample and out through the electrical grounding of the specimen holder. In the case of polymers, this is not the case. Instead, electrons accumulate on the surface and create an electric field that deflects the secondary electrons that are critical to produce a high resolution image. Normally, coating the surface with a very thin layer (in the Angstrom scale) of metal, such as gold or platinum, fixes this charging effect. However, for a bulk polymer material, such as PDMS, this coating step alone must be tweaked to achieve decent images. An in-detail explanation of a proposed solution that successfully helped in characterizing the molded structures is demonstrated in Appendix C.

To demonstrate the reliability and mold robustness of this method, the same Si master mold was used several times and the procedure explained above was repeated for at least 5 times to mold 5 different PDMS samples with sub-micron PhC lattices of air holes. Figure 4.29 demonstrate SEM images of the Si master mold after the 5 molding experiments while Figure 4.30 demonstrates SEM images of the 5 molded PDMS PhC samples. It is obvious that even after 5 molding experiments, the master mold can still be reused and no sign of PDMS traces were identified.

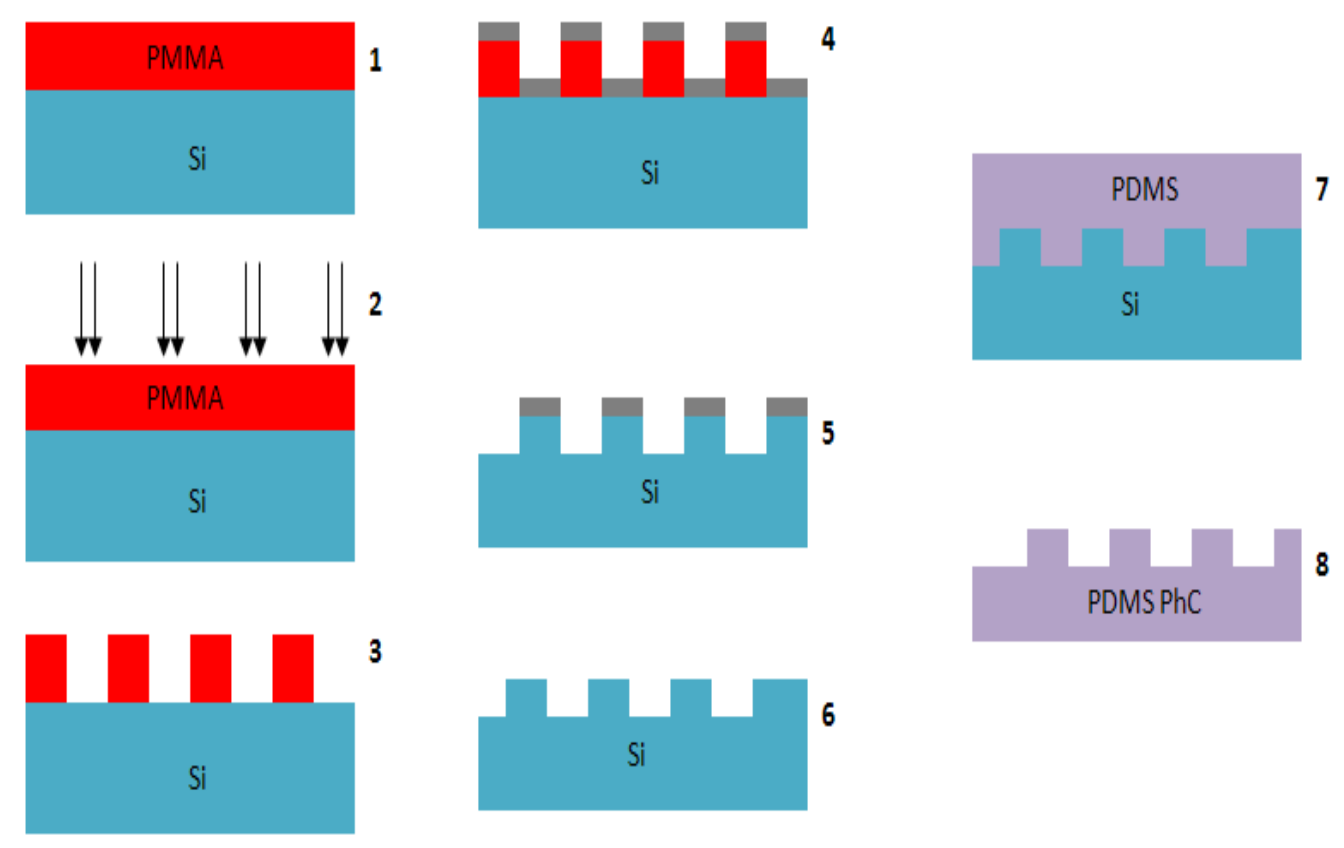

Figure 4.26. Fabrication procedure to achieve a final photonic crystal lattice of air holes in PDMS. (1) PMMA spin coating, (2) e-beam lithography, (3) PMMA development, (4) Ni thin mask deposition, (5) Ni lift off and dry etch of Si, (5) removal of Ni mask. (6) PDMS molding, (7) final PhC structure in PDMS 


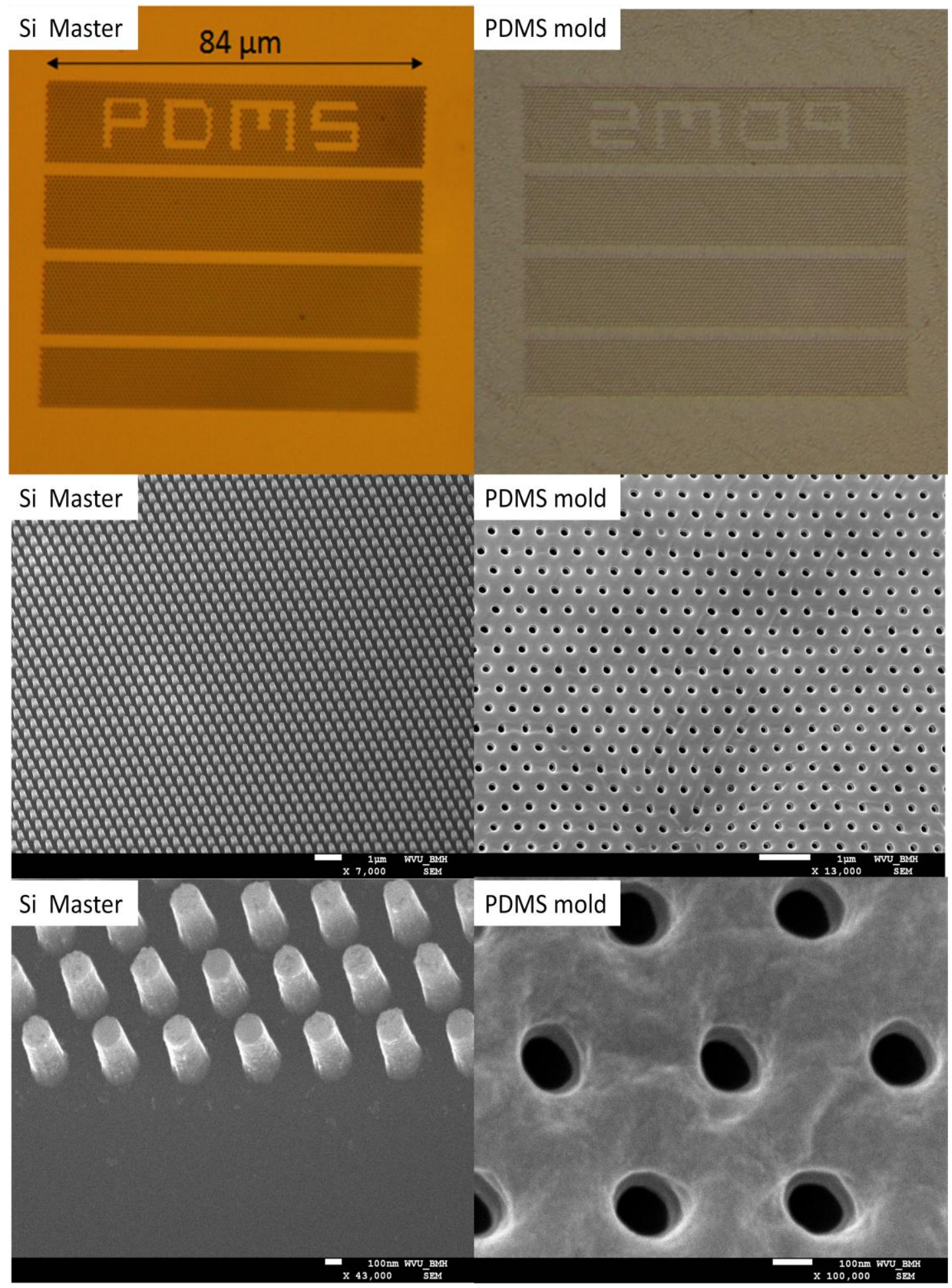

Figure 4.27. Optical and SEM images of the Si master (left column images) and the molded PDMS sample (right column images) containing the sub-micron $\mathrm{PhC}$ lattice. 


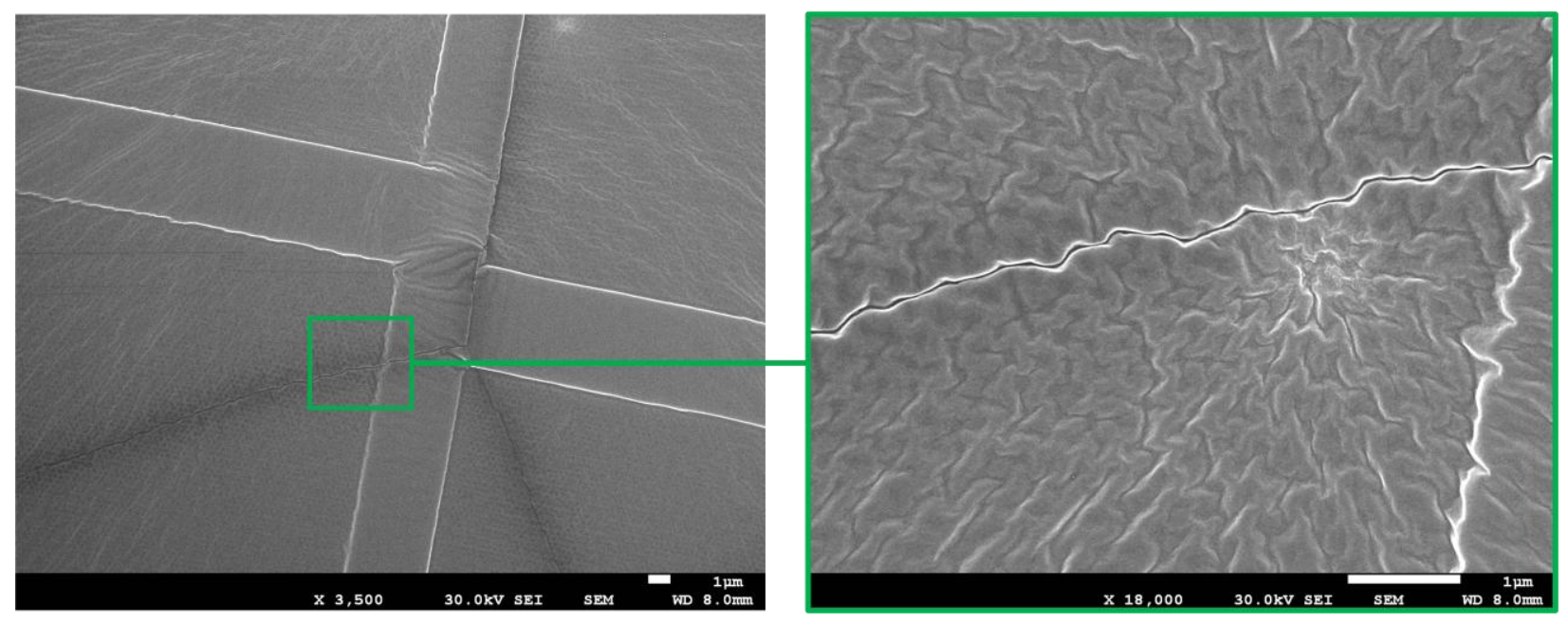

Figure 4.28. Un-resolved $\mathrm{PhC}$ lattice of air holes due to the use of low vacuum during the degassing step

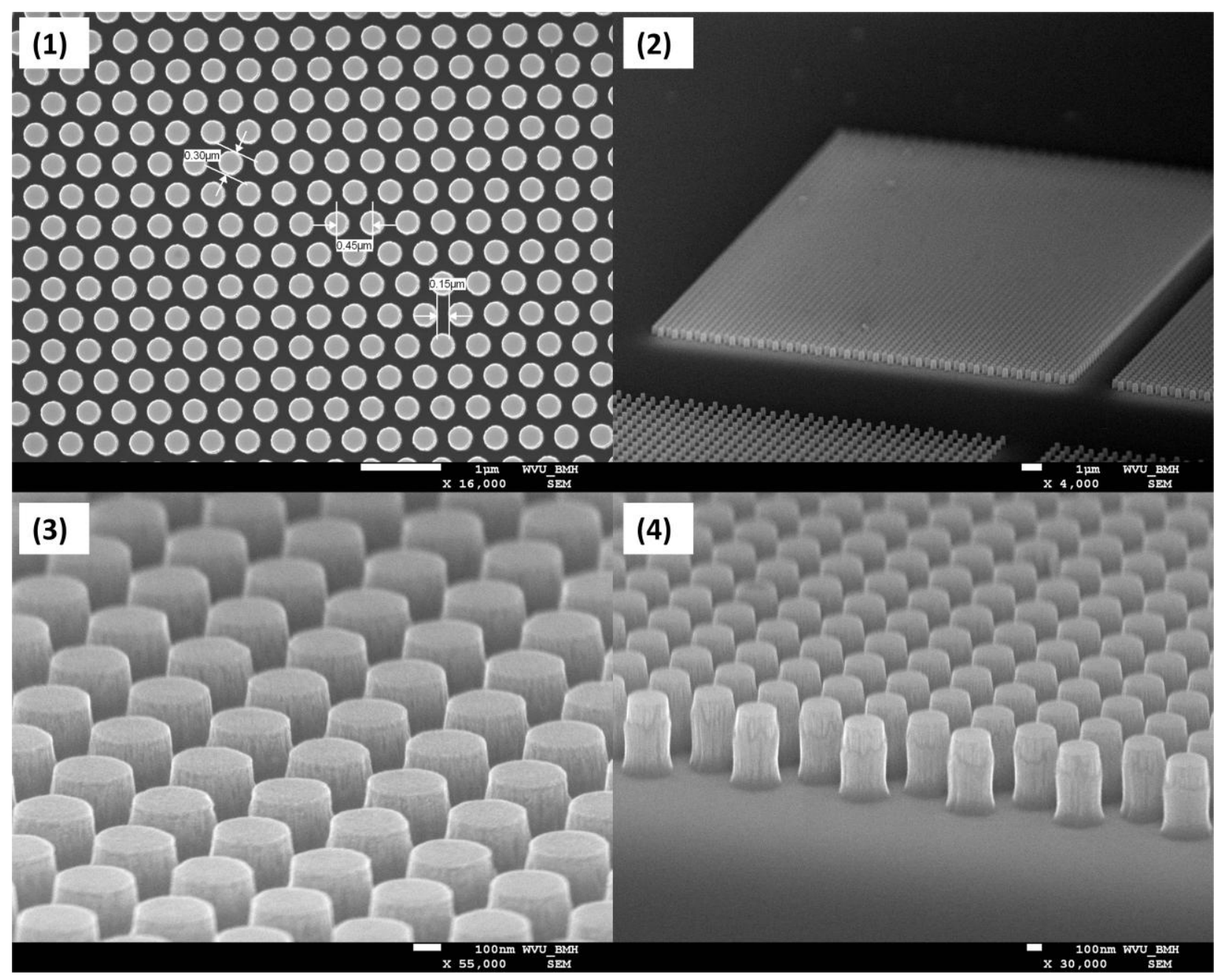

Figure 4.29. SEM images of the Si master mold containing pillars with $\mathrm{r}=150 \mathrm{~nm}, \mathrm{a}=450 \mathrm{~nm}$ (spacing of $150 \mathrm{~nm}$ ), and $\mathrm{h}=460 \mathrm{~nm}$. (1) top view. (2) tilted image of the full block, (3) the tips of the Si pillars, and (4) the full Si pillars 


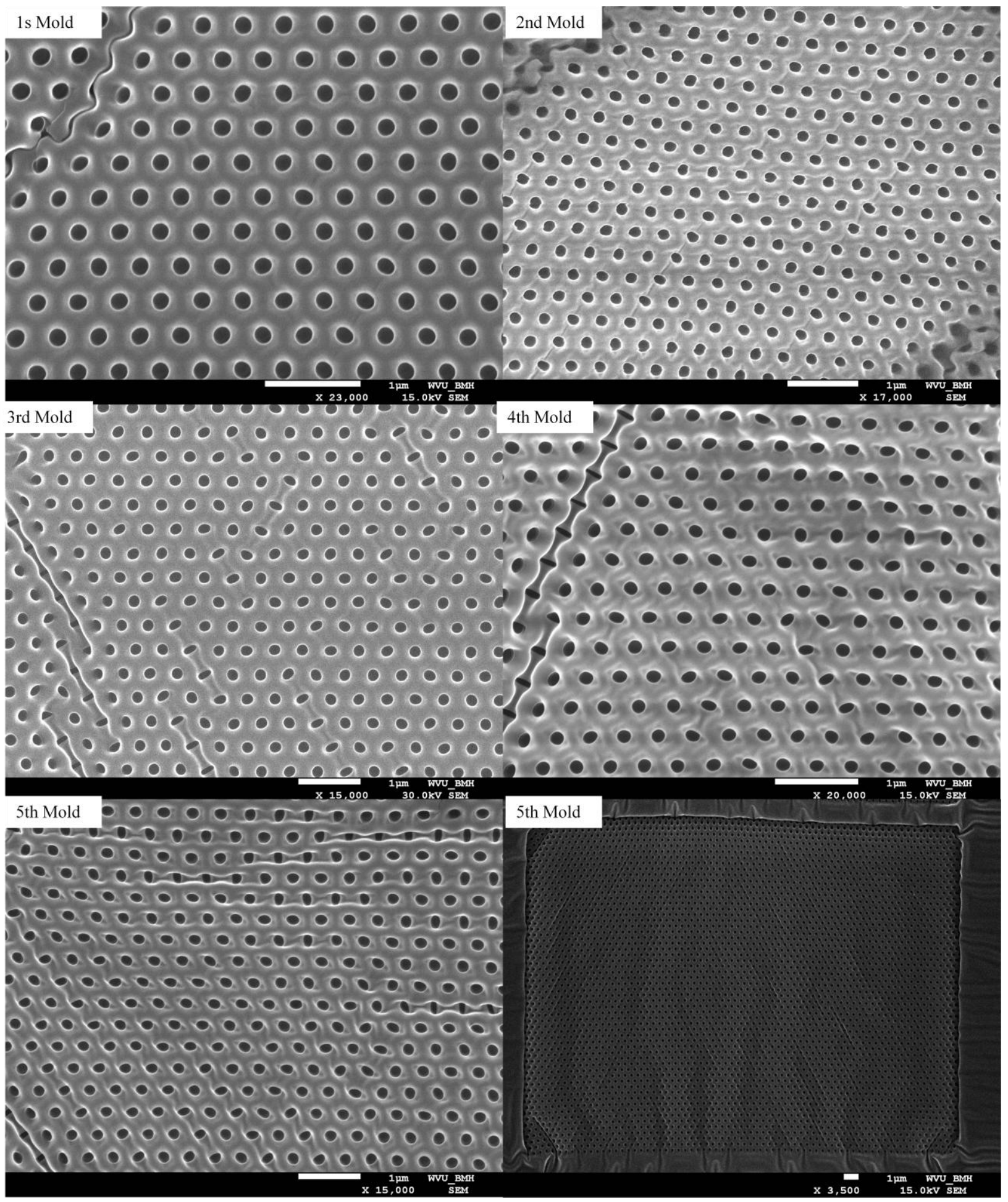

Figure 4.30. SEM images of the 5 molded PDMS samples using a single Si master.

Observed distortions are due to charging effects and cracking of PDMS during the sputtering step. For $5^{\text {th }}$ mold, a top view of the full pattern area is shown 
As explained earlier, capturing the SEM images shown in Figure 4.30 was a challenging task due to charging effects. However, sputtering approximately $40 \mathrm{~nm}$ of Platinum using the procedure explained in Appendix $\mathrm{C}$ helped to significantly reduce this effect. Never the less, some charging effect combined with the cracking of the thin Ni layer on top of the flexible PDMS sample when mounting it on the SEM holder upon imaging caused the obvious distortion in the shape of the circular holes. The diameter of several perfect circular holes in each mold was recorded. These dimensions are tabulated in Table 4.3. Approximately for all of the 5 molds, the diameter of the holes was around $170 \mathrm{~nm}$ which is $130 \mathrm{~nm}$ less than the diameter of the pillars in the master mold. This big deviation in the diameter can be attributed to the thick layer of Ni that was deposited to properly characterize the PDMS sample using electron-beam microscopy. Moreover, shrinkage of the PDMS sample upon its release from the master is also expected to play a significant role in affecting the resolution of this soft lithographic technique.

Table 4.3. The actual dimensions of the diameter of the mater Si pillars and of the holes of the 5 molded PDMS PhC structures

\begin{tabular}{r|l|l|l|l|l|l|l|}
\multicolumn{1}{c|}{$\begin{array}{c}\text { Si } \\
\text { Master }\end{array}$} & \multicolumn{9}{c}{ PDMS } \\
\hline & Mold\# 1 & Mold\# 2 & Mold\# 3 & Mold\# 4 & Mold\# 5 & Average Diameter & Percent Deviation \\
\hline $300 \mathrm{~nm}$ & $177 \mathrm{~nm}$ & $171 \mathrm{~nm}$ & $167 \mathrm{~nm}$ & $174 \mathrm{~nm}$ & $172 \mathrm{~nm}$ & $172.2 \mathrm{~nm}$ & $42.2 \%$ decrease \\
\hline
\end{tabular}

\subsubsection{Epoxy-based Photonic Crystals}

Another polymer material in which photonic crystal lattices fabricated in the nano-scale would be desirable is epoxy. Epoxy has a higher refractive index than PDMS and therefore, its partial bandgap can be designed to be wider than that for PDMS. Biocompatible thermally curable epoxy is commercially available from Epoxy Technology®. More specifically, the soft lithographic study conducted here is mainly done on the EPO-TEK 301-2 Optical Epoxy. It is a two-component biocompatible and non-toxic epoxy that is commonly incorporated in the fabrication of Lab-on-a-Chip systems. In this section, the ability to produce nano-scale high-density photonic crystal structures that can potentially become core biocompatible structure of an ultra-sensitive photonic crystal-based biosensor is demonstrated.

The ability to resolve bandgaps for epoxy allows their incorporation in biosensors utilizing both the active and passive modalities. In the passive modality, the epoxy PhC slab can replace the Si slab and resonant cavities can be tuned for frequencies that fall within the bandgaps to observe fluorescence enhancement. As for the active modality, epoxy $\mathrm{PhC}$ structures can act as narrow transmission or reflection structures that can be tuned to display shifts when target analytes adsorb to the surface of a high $\mathrm{RI}$ material deposited on them [26] or to walls of the $\mathrm{PhC}$ pores [24]. 
In order to achieve sub-micron features in epoxy, the procedure is a little more complex than that for PDMS. The reason is mainly due to the fact that epoxy, once cured, becomes highly adhesive to the Si master mold and peeling it off becomes an impossible task. In order to overcome this obstacle, PDMS can act as an intermediary master which is easily peeled off the epoxy molded structure. However, this means that the PDMS structure should contain the inverse pattern of what is desired to be finally produced in epoxy. This also means that the starting Si mold should have the exact same pattern as that of epoxy. For example, to produce epoxy pillars, the Si master mold must also contain Si pillars which will in turn produce PDMS holes that act as a master when molding the epoxy.

To produce a final sub-micron PhC lattice of epoxy pillars surrounded by air, the fabrication procedure to produce the Si master of pillars surrounded by air is explained in section 4.5 .2 and the molding procedure of the intermediary PDMS mold is explained in section 4.7.1. Once the PDMS mold with air holes was generated, Part A of the EPO-TEK 301-2 Epoxy was mixed with part B in a 100:35 ratio for two minutes at $2000 \mathrm{rpm}$. Few droplets of the epoxy solution were then poured on the PDMS region that contains the inverse photonic crystal structure. The Petri dish containing the PDMS master and the top liquid epoxy were then put inside a desiccator connected to an XD-5 Edwards scroll pump for a degassing step for 5 minutes. At the end of this period, to remove the air bubbles that accumulate on the surface of the epoxy, slow vacuum release was done. It is important to note that degassing of Epoxy is a relatively harder task than that for PDMS. Therefore, it is possible that some air bubbles would still exist in the epoxy mold at the end of the degassing period.

At this stage, the Petri dish was transferred to an oven set to 60 degrees and left to cure for at least 5 hours. The Petri dish was left to cool down and then the hard clear epoxy was peeled off the PDMS master. Figures 4.31 and 4.32 demonstrate this soft lithography fabrication procedure and descriptive SEM images of the initial molded epoxy PhC samples. When the final degassing step was done using house vacuum, the molded epoxy PhC structures were not fully resolved as shown in Figure 4.33. The process explained above was repeated 5 times using the same PDMS master mold sample. SEM images of the successful molding results after each step are demonstrated in Figure 4.34.

As explained in the previous section, analyzing the molded structures using SEM was a challenging task due to charging effects. However, sputtering approximately $20 \mathrm{~nm}$ of Platinum using the procedure explained in Appendix $\mathrm{C}$ helped to significantly reduce this effect. Never the less, some charging effect still caused the obvious distortion in the shape of the circular pillars. The diameter of several near-toperfect pillars in each mold was recorded. These dimensions are tabulated in Table 4.4. Approximately for all of the 5 molds, the diameter of the holes was around $296 \mathrm{~nm}$ which is only $4 \mathrm{~nm}$ less than the original master mold and $20 \mathrm{~nm}$ less than the intermediate PDMS master mold. These results are much closer to the target diameter due to the fact that the intermediate PDMS mold was not sputtered and 
therefore, the size deviation that was observed in the previous section will not have an effect on the molded epoxy samples. However, shrinkage or expansion of the molded structures can still attribute to the deviation of the dimensions from their designed targets.

Table 4.4. The diameter of the mater master mold features and of the pillars of the 5 molded epoxy PhC structures

\begin{tabular}{|c|c|c|c|c|c|c|c|c|}
\hline \multirow[t]{2}{*}{ Si Master } & \multirow[t]{2}{*}{ PDMS Master } & \multicolumn{7}{|c|}{ Epoxy } \\
\hline & & Mold\# 1 & Mold\# 2 & Mold\# 3 & Mold\# 4 & Mold\# 5 & Average Diameter & Percent Deviation \\
\hline $300 \mathrm{~nm}$ & $316 \mathrm{~nm}$ & $295 \mathrm{~nm}$ & $294 \mathrm{~nm}$ & $290 \mathrm{~nm}$ & $310 \mathrm{~nm}$ & $294 \mathrm{~nm}$ & $296.6 \mathrm{~nm}$ & $1.14 \%$ \\
\hline
\end{tabular}
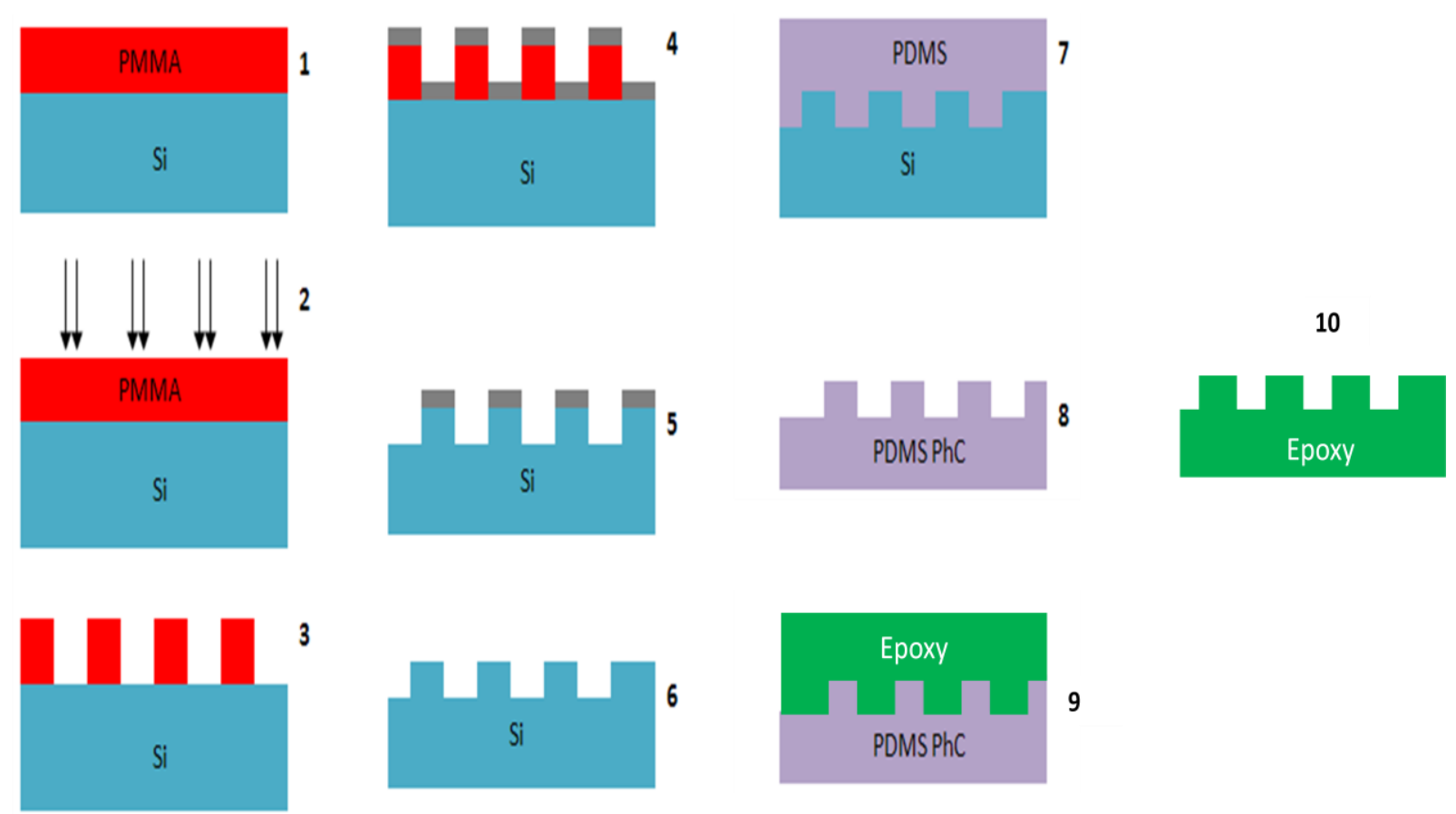

Figure 4.31 (1) PMMA resist spin coating on the first Si mold, (2) E-beam Lithography, (3) Development of the PMMA resist, (4) Ni mask deposition using E-beam Evaporation, (5) Ni mask lift off and dry etch of the Si substrate, (6) Removal of the Ni mask layer, (7)-(8) molding of the PDMS to create the inverse pattern, (9) Pouring Epoxy on the PDMS master and curing it in an oven set to 60C for 5 hours (10) Final PhC structure in Epoxy 


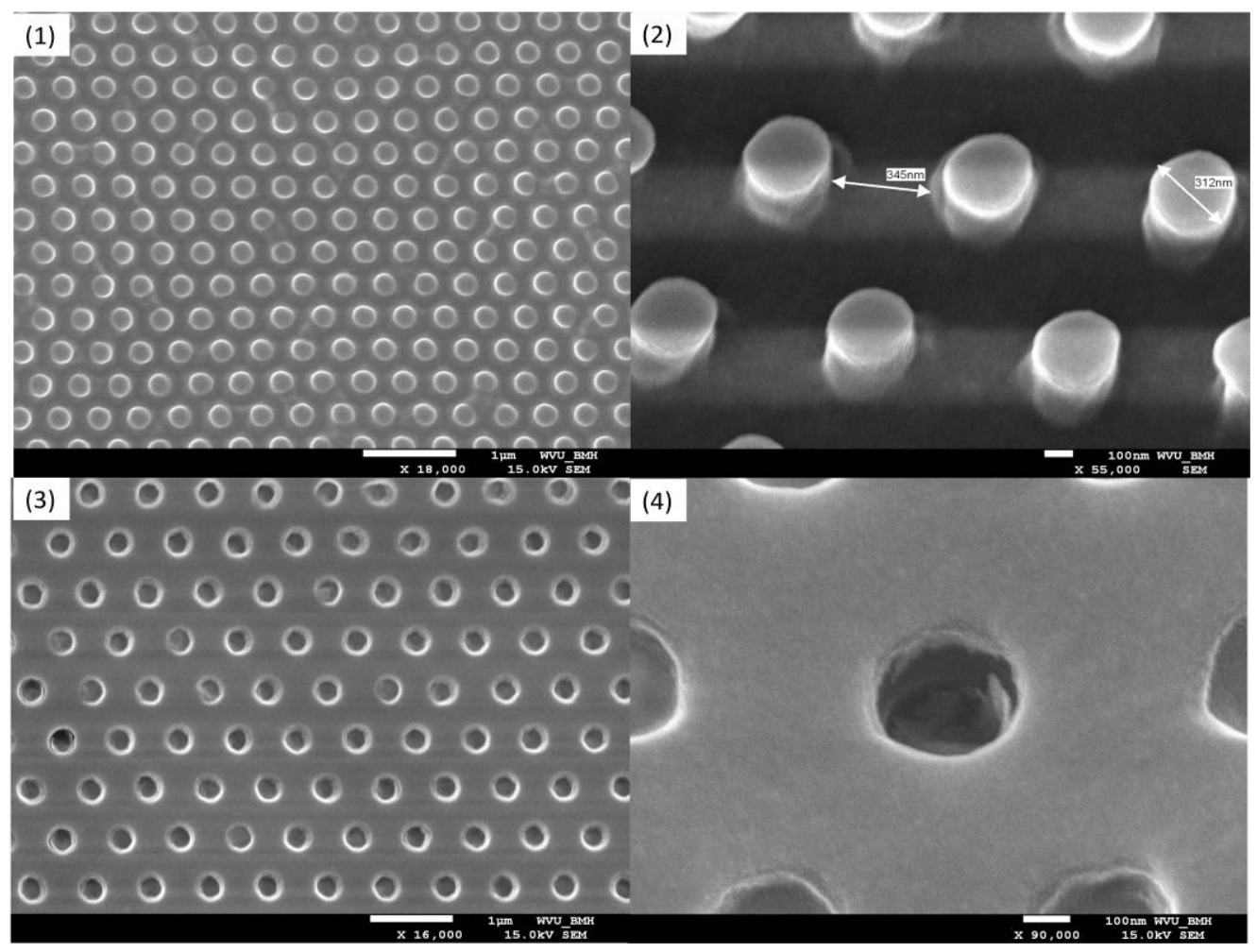

Figure 4.32 (1)-(2) SEM images of the final sub-micron PhC lattice of epoxy pillars surrounded by air, (3)-(4) SEM images of the final sub-micron PhC lattice of air holes in epoxy when a Si master with holes is used instead of pillars
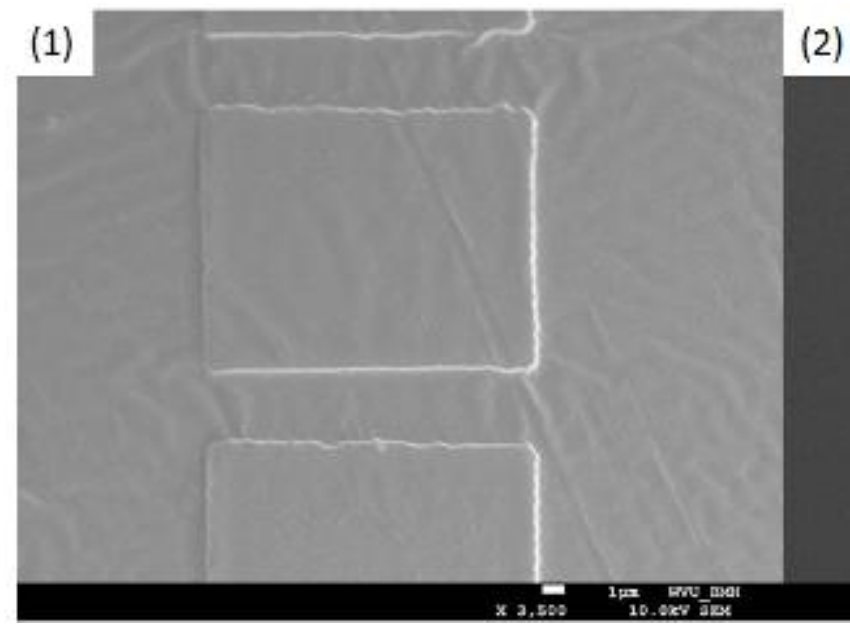

Figure 4.33 (1) Un-resolved epoxy pillars due to the use of low vacuum during the degassing step (2) resolved Epoxy pillars due to the use of a XD-5 Edwards scroll pump during the degassing step 


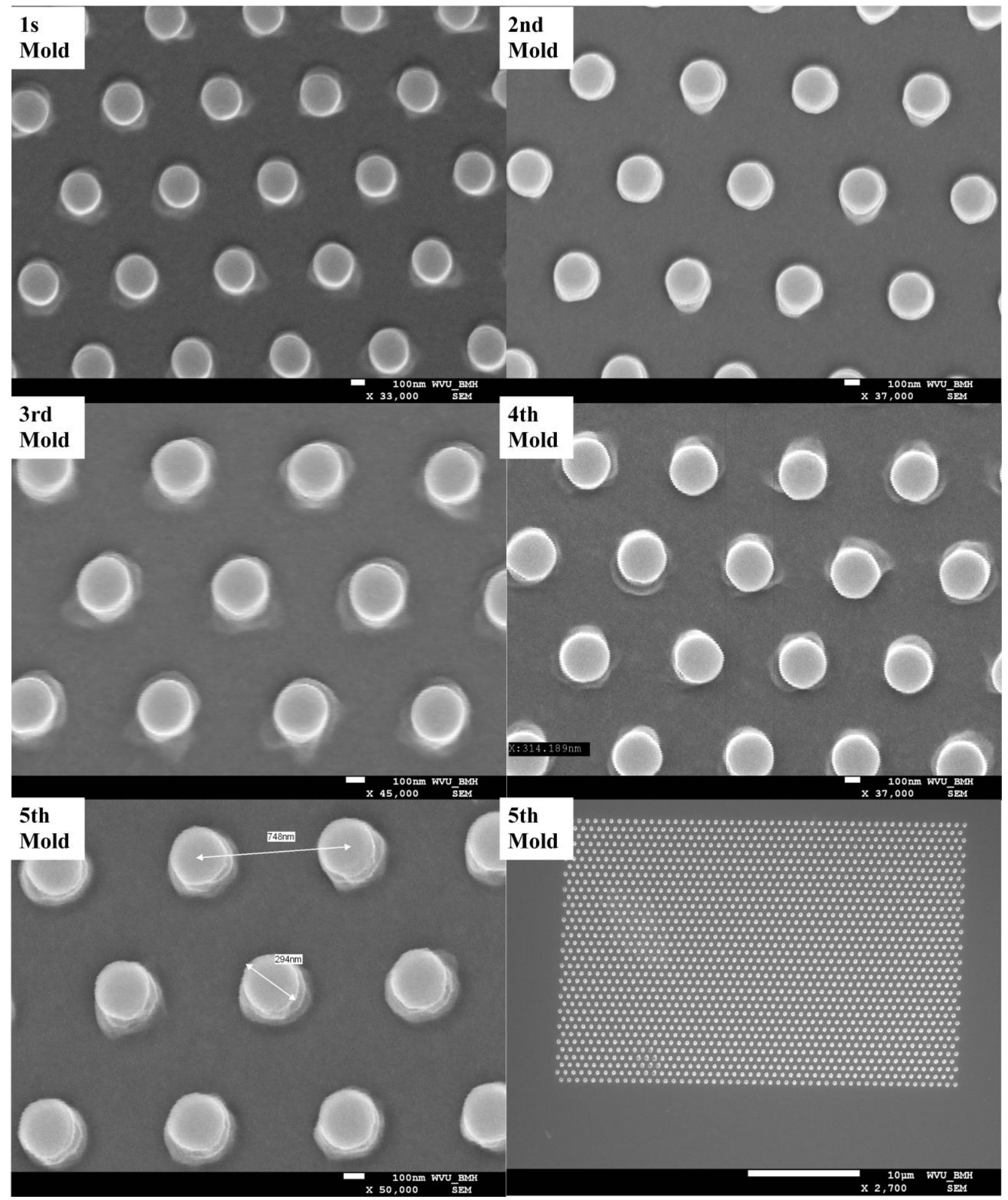

Figure 4.34. SEM images of the 5 molded epoxy samples using a single Si and PDMS master molds. Observed distortions are mainly due to charging effects. For the $5^{\text {th }}$ mold, a top view of the final molded PhC area is shown 


\section{CHAPTER 5: OPTICAL CHARACTERIZATION}

In the previous chapters, the effect of periodic photonic crystal lattices on propagating electromagnetic waves has been discussed. The periodically varying dielectric constant of the photonic crystal slab prohibits a specific range of wavelengths from propagating through the lattice. Therefore, if a labeled molecule becomes trapped within a slab $\mathrm{PhC}$ and starts to emit light that falls within the bandgap of this lattice, a confinement of this emission is expected to be observed. Without resonant defect cavities, this confinement should exist only within the $\mathrm{PhC}$ region and decays in the background surrounding the lattice. Once a resonant cavity is introduced and the labeled molecules are forced to emit only from inside those cavities, then spatial confinement of the emission can be localized in the resonant cavity and larger enhancement factors are possible due to the fact that the low modal volume cavities can potentially lead to higher emission rates as theorized by the Purcell effect [76,77].

An optical characterization experiment was designed in order to prove that slab photonic crystals can prohibit in-plane propagation of wavelengths that fall within its bandgap and induce an enhancement of this emission. The experiment centers on nano-scale molecules that can emit a specific wavelength or range wavelengths that fall within the photonic bandgap, specifically, $\mathrm{PbS}$ Quantum Dots obtained from Evident Technologies. Characteristics of these quantum dots are summarized in Table 5.1.

Table 5.1. Characteristics of the IR-emitting QDs that were used in the optical characterization experiment of the PhCs (Evident Technologies)

\begin{tabular}{|c|c|}
\hline \multicolumn{2}{|c|}{ EviDot Certificate of Analysis } \\
\hline Product: & EviDot core \\
\hline Material: & $\mathrm{PbS}$ \\
\hline Lot \#: & LNP16JPB \\
\hline Emission Peak (nm): & 1125 \\
\hline Absorption Peak (nm): & 990 \\
\hline Emission Peak FWHM (nm): & 141 \\
\hline $\begin{array}{r}\text { nanocrystal diameter }(\mathrm{nm}) \text { : } \\
\text { (crystalline core) }\end{array}$ & 3.2 \\
\hline $\begin{array}{l}\text { total particle diameter }(\mathrm{nm}): \\
\text { (arstalline core + est of ligands) }\end{array}$ & 7.2 \\
\hline $\begin{array}{r}\text { molar extinction coeff: } \\
\text { (based on core data) }\end{array}$ & $6.2 \mathrm{E}+04$ \\
\hline $\begin{array}{r}\text { nanocrystal MW } \\
\text { (crystalline core) }\end{array}$ & $2.0 \mathrm{E}+04$ \\
\hline $\begin{array}{c}\text { particle MW ( } \mu \mathrm{g} / \mathrm{nmol}): \\
\text { (cystalline core + est of ligands) }\end{array}$ & $8.0 \mathrm{E}+04$ \\
\hline
\end{tabular}


The $141 \mathrm{~nm}$ FWHM indicates that these QDs are not monodispersed (monosized) particles. Their size distribution is normally calculated by either modeling a specific approximate size distribution surrounding the emission peak and then fitting that modeling result to emission spectra, or simply by carrying out Transmission Electron Microscopy measurements. These nanoparticles are very desirable for our experiment due to two main reasons:

1) The wide separation between the excitation and emission peaks of tens or hundreds of nanometers allows the design of a PhC lattice where the excitation beam can fall outside of the bandgap and therefore is not expected to be enhanced while only enhancing the emitted light. Using an Applied Nanofluorescence NS1 NanoSpectralyzer, the absorption and emission spectra were measured for a clear diluted sample of the Quantum Dot solution. The absorption and emission spectra are demonstrated in Figure 5.1.

2) Moreover, the $\mathrm{PbS}$ quantum dots are approximately $7 \mathrm{~nm}$ in diameter suspended in the low viscous toluene solvent. This will allow the suspended QDs to flow through the $\mathrm{PhC}$ slab, at least through a slab of high dielectric rods as will be discussed later in this chapter.

In Figure 5.1, the peak emission of the IR-emitting QDs is around $1100 \mathrm{~nm}$ and therefore, any lattice with partial, directional, or complete photonic bandgap must be designed to have this emission to fall within the bandgap. When the QDs emit from within the photonic crystal slab, the emission is expected to be confined to the boundaries of the slab as long as it falls within the in-plane photonic bandgap. If the $\mathrm{PhC}$ lattices is made of at least 14 periods, as was discussed in Chapter 3, then leakage of this emission is expected to be only in the vertical direction above and below the slab.

In order to perform the experiment, an Olympus inverted microscope setup was used as shown in Figure 5.2. A droplet of the QD solution was placed on a thin glass slab on the microscope and a PhC sample was flipped and slowly placed on the droplet. This allowed the PhC lattice on the top surface of the SOI or Si sample to become immersed by the toluene solvent where the QDs exist in suspension. The inverted optical microscope setup focuses a $250 \mathrm{~mW} 514 \mathrm{~nm}$ excitation beam on the $\mathrm{PhC}$ region to excite the QDs that are within and above the slab. A dichroic mirror with filter cutoff frequency of $950 \mathrm{~nm}$ is placed in the path of the emitted light to allow only the desired wavelengths to pass to a Goodrich shortwave infrared (SWIR) camera 

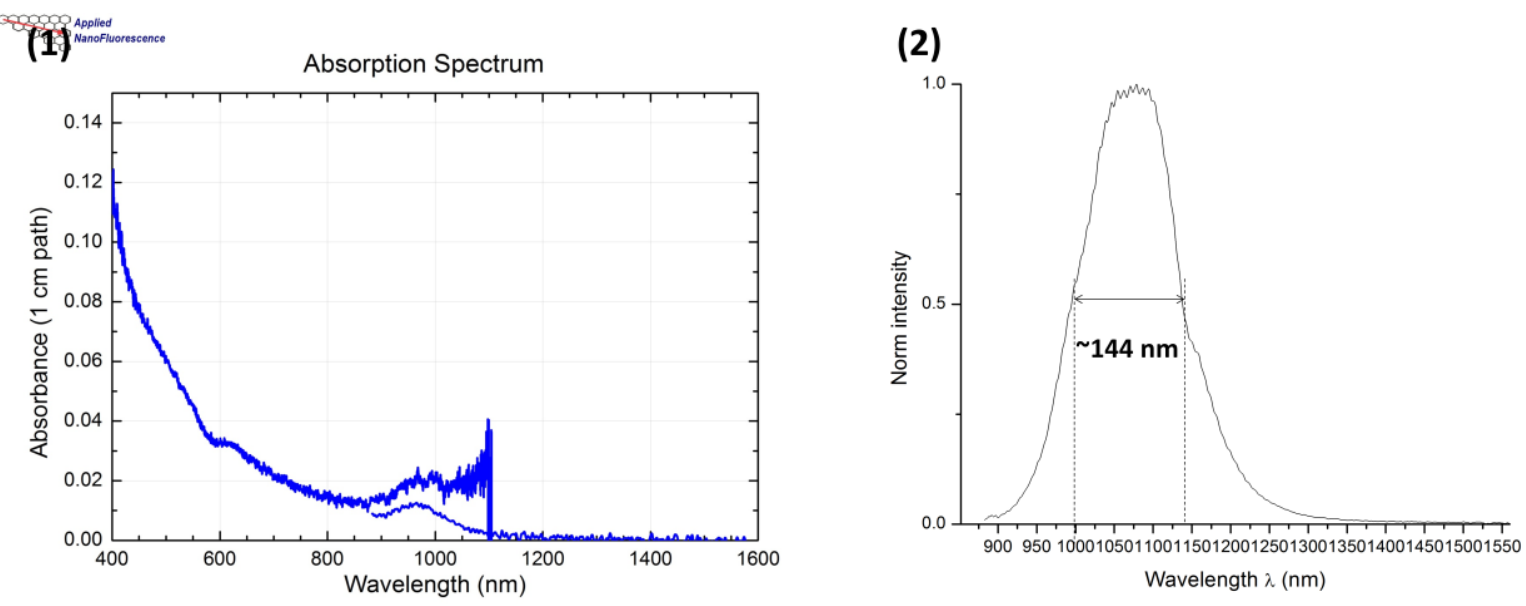

Figure 5.1. The absorption (1) and emission spectra (2) of the PbS QDs

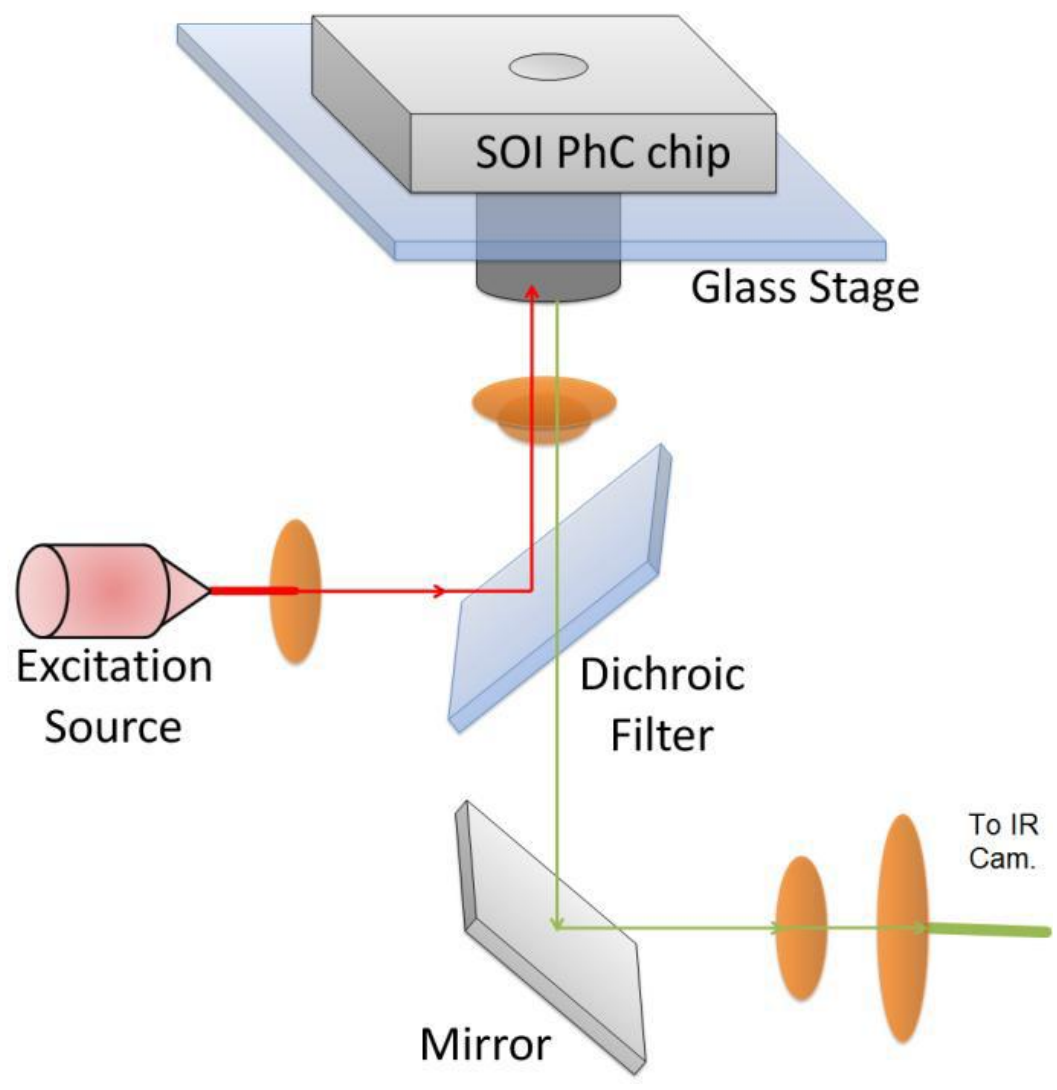

Figure 5.2. Optical setup to observe fluorescence enhancement 


\subsection{CHARACTERIZATION OF A FINITE-THICKNESS SLAB OF A TRIANGULAR LATTICE OF TOLUENE-FILLED HOLES IN SILICON}

Fabricating suspended photonic crystal triangular lattices of air holes in $\mathrm{Si}$ was performed as explained in section 4.5.1. The optical characterization experiment was conducted on a sample with radius $r=0.35 a$ and height $h=0.9 a$. The band diagrams of an infinitely thick representation of this lattice as well as a finite-thickness representation are shown in Figure 5.3. In both diagrams of Figure 5.3, a bandgap can be observed. In the infinitely thick slab of triangular toluene-filled holes within $\mathrm{Si}$ demonstrates a complete TE bandgap. TM bandgaps for this lattice do not exist. On the other hand, for a finite-thickness slab of toluene-filled holes in $\mathrm{Si}$, only a directional bandgap in the $\mathrm{M}$ to $\mathrm{K}$ direction can be observed. If the toluene holes are replaced with air, then a complete bandgap is observed. However, it is important to consider the real setup in which the PhCs are expected to operate, so it makes sense to only consider PhC slabs in toluene as the air condition is an ideal case that will not be practical to our application.

The band diagram of a slab photonic crystal with $\mathrm{r} / \mathrm{a}=0.35$ and $\mathrm{h} / \mathrm{a}=0.9$ shows that the normalized frequency 0.25 falls within the bandgap. Designing the lattice parameters for QD emission of $1100 \mathrm{~nm}$ to be within this bandgap and represent this frequency results in the lattice constant (a) of $\sim 279 \mathrm{~nm}$, lattice hole radius (r) of $\sim 98 \mathrm{~nm}$, and slab thickness (h) of $\sim 251 \mathrm{~nm}$. This thickness is very close to the original SOI top layer Si thickness of around $260 \mathrm{~nm}$. Once these dimensions were determined, a DesignCAD file was created. It is important to distinguish PhC bandgap effect enhancement due to in-plane confinement from that of regular QD aggregation inside the PhC. Therefore, a lattice with parameters that fall below the desired bandgap and another with no bandgap are all fabricated in close proximity to the one with a bandgap. Figure 5.4 demonstrates SEM images of the different fabricated structures.
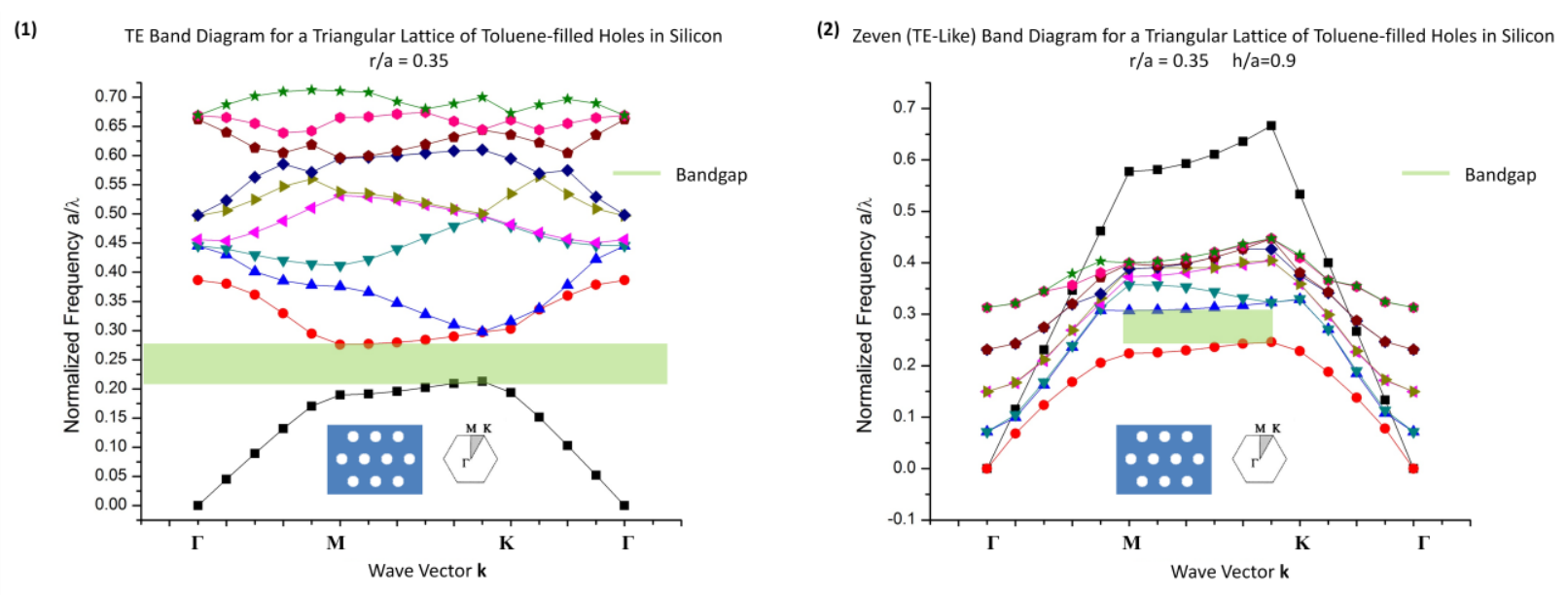

Figure 5.3. Band diagrams of (1) an infinitely-thick $\mathrm{PhC}$ and (2) finite-thickness slab with $\mathrm{r} / \mathrm{a}=0.35$ and h/a=0.9 


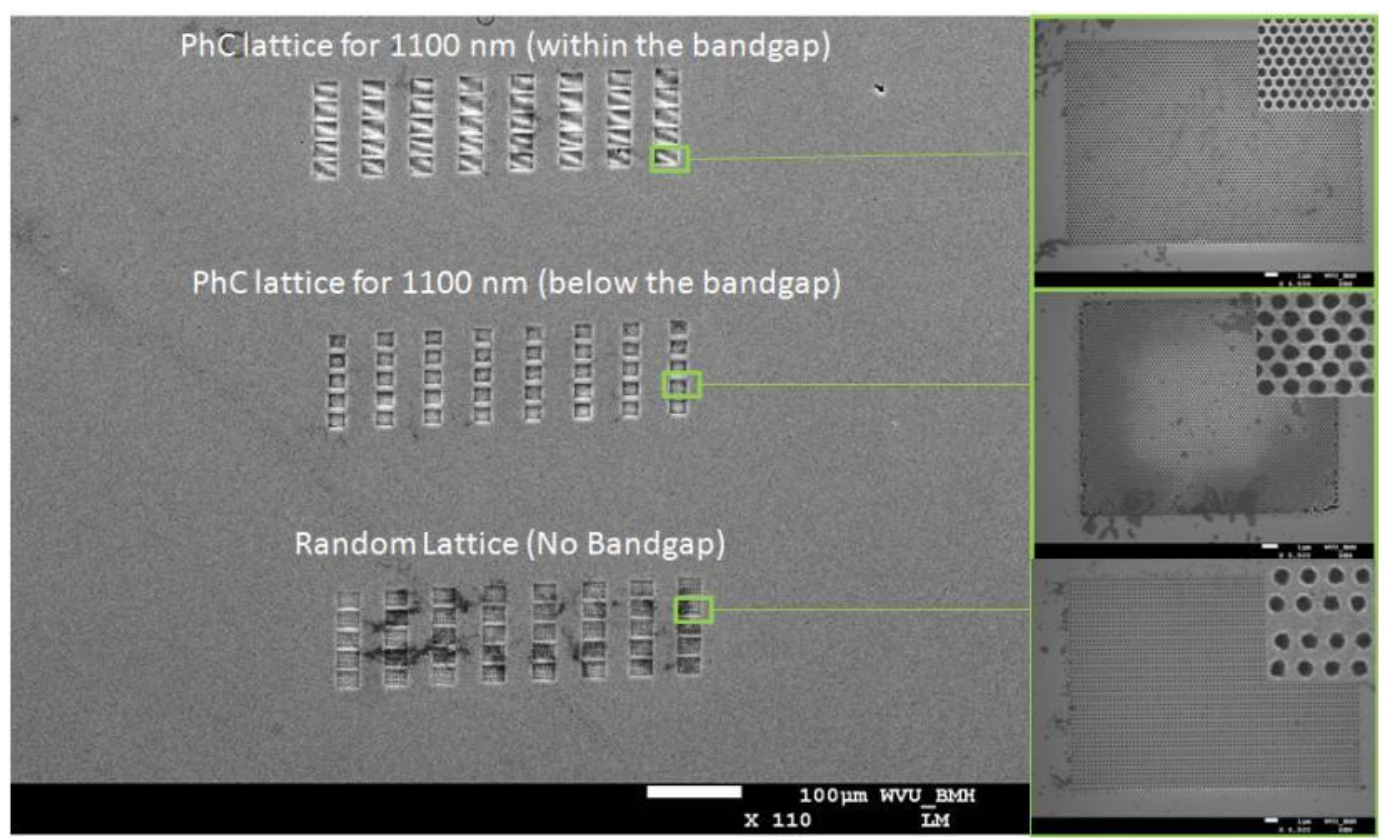

Figure 5.4. SEM image of the suspended PhC structures. Top rows are identical PhC lattices designed for $1100 \mathrm{~nm}$ to be within their bandgap. Middle row are identical PhC lattices in which the $1100 \mathrm{~nm}$ emission falls below their bandgap. Bottom row demonstrates a repeated, identical random lattice with no bandgap.

Moreover, the structures of Figure 5.4 are fabricated twice. The first time, these structures are fabricated on a SOI wafer to allow for a symmetric material architecture for the PhC lattice, which is very critical in setting up complete photonic bandgaps for the guided modes within the slab. The second time, these structures are fabricated on a regular $\mathrm{Si}$ wafer, creating an asymmetric architecture with the $\mathrm{Si}$ substrate below the PhC structure and toluene within it and above it. This break of symmetry will cause the modes to be couple into the bulk semiconductor region below the slab, and no bandgaps are expected to be observed for either lattice.

When the experiment was conducted while the quantum dots solution is still present, both the symmetric and asymmetric lattices appeared dark with no noticeable distinguishing enhancement effect for the lattices with a photonic bandgap. Figure 5.5 illustrates the optical measurement results side by side for both the SOI and Si samples. It can be observed that all three structures are darker than the surrounding background, even when background fluorescence is removed. This is the opposite of what was expected to be observed for the SOI PhC lattice with a photonic bandgap that surrounds the $1100 \mathrm{~nm}$ emission. However, it is important to mention that the SOI PhC lattices are suspended in air. It is possible that the trapped air underlying the slab prevents the toluene-suspended QDs from flowing through the slab. Moreover, if the QDs do emit from within the slab, then using a high dielectric slab with low dielectric holes, the confinement of the emitted light is expected remain within the slab as the suspended 
$\mathrm{PhC}$ lattices with vertical symmetry are normally fabricated as optical waveguides. This again will prevent the observation of any enhancement.

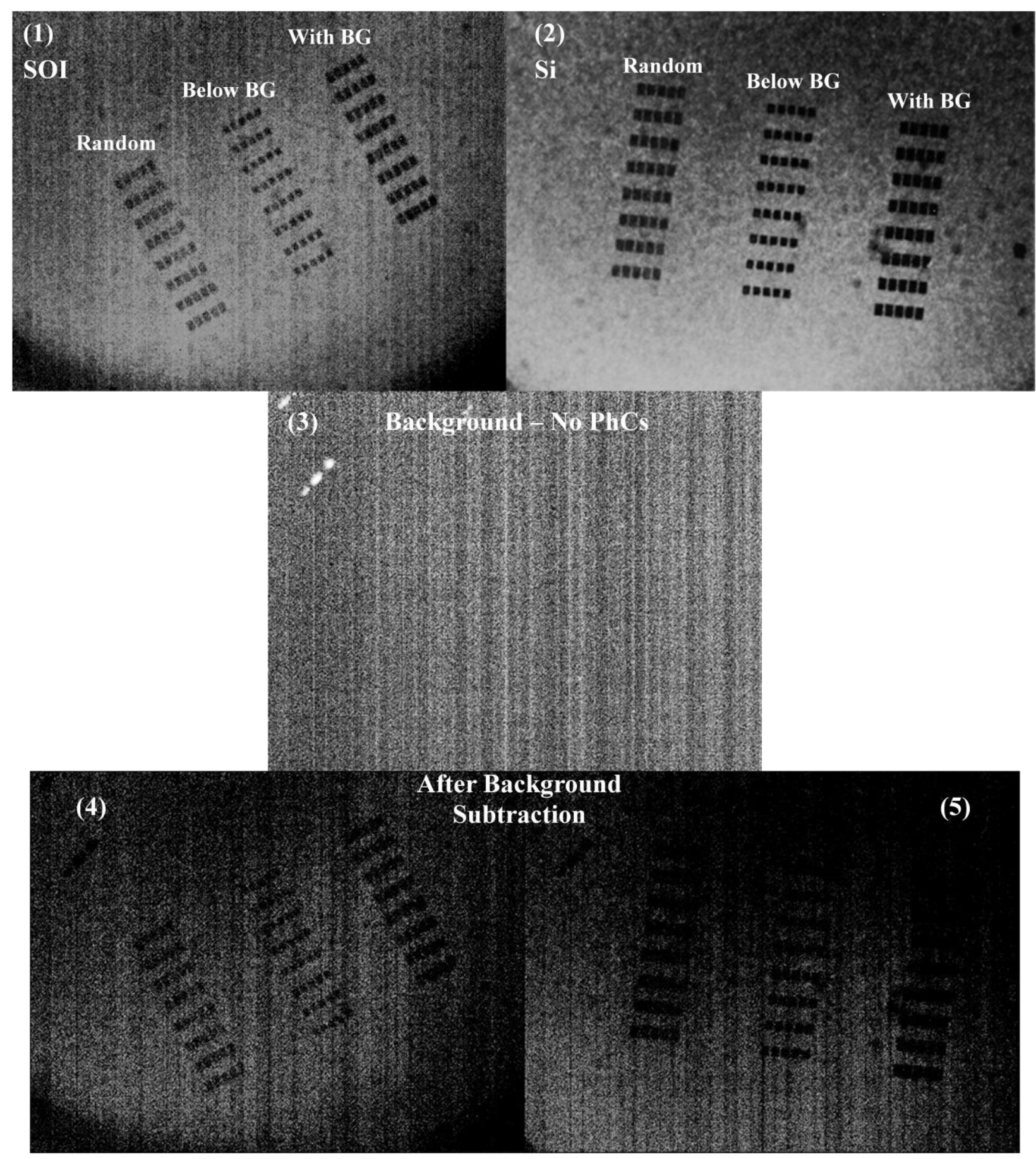

Figure 5.5. Optical characterization results of a (1) suspended and (2) an un-suspended $\mathrm{PhC}$ lattices of holes in $\mathrm{Si}$ fabricated in SOI and Si samples, respectively. (3) The background image of an area close to the PhC lattice. (4) SOI after background subtraction. (5) Si (unsuspended) after background substraction 


\subsection{CHARACTERIZATION FINITE-THICKNESS PHOTONIC CRYSTAL SLABS OF A TRIANGULAR LATTICE OF SI PILLARS SURROUNDED BY TOLUENE}

In order to overcome the obstacle observed in the previous section when characterizing PhC slabs of holes, an inverse lattice of pillars surrounded by low dielectric background is advised. This structure is expected to be more suitable for wet experiments since the toluene-suspended quantum dots will be able to flow easier through the plane of pillars, and there will be no need to force them to flow into small volumes as in the case with slabs of air holes. However, to create a PhC lattice with suspended pillars surrounded fully by toluene is not possible. There must be something to support the pillars. It has been previously theoretically demonstrated that once the $\mathrm{PhC}$ structure becomes 'extruded' to a layer that separates it from the bulk material, then photonic bandgaps for the guided modes within this slab can be observed [**]. When using a SOI wafer, this extrusion extends from the top Si layer to the underlying sacrificial $\mathrm{SiO}_{2}$ layer.

To achieve this structure, the fabrication process detailed in section 4.5.2 was used to fabricate pillars of Si in the top Si layer of an SOI wafer. However, in order to lower the effect of the refractive index of the underlying $\mathrm{SiO}_{2}$ layer, the $\mathrm{PhC}$ structure is etched for a longer period and therefore a lower effective refractive index can be introduced when the whole structure becomes immersed in the toluenesuspended QDs. The band diagrams of an infinitely thick structure of Si pillars in toluene and that of a finite-thickness slab of Si pillars suspended by an effective refractive index of toluene are shown in Figure 5.6.

(1)

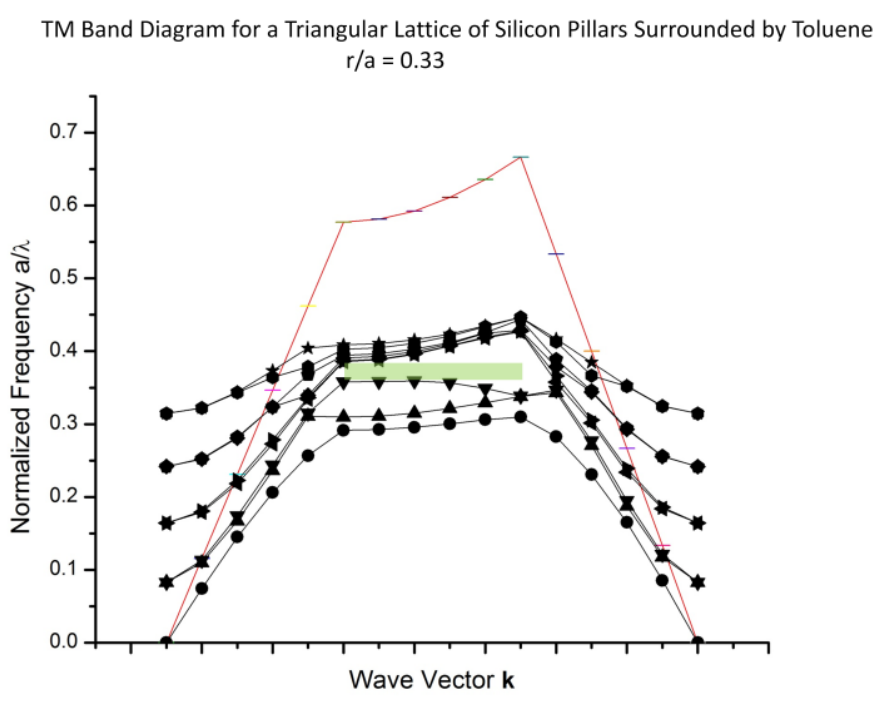

(2)
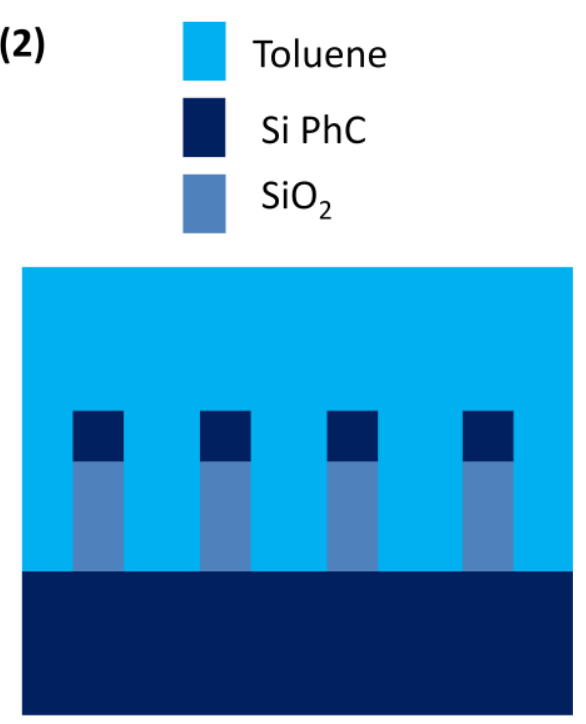

Figure 5.6. (1) The band diagrams of a finite-thickness slab of Si pillars surrounded by toluene background (2) A two dimensional cross-section representation of the $\mathrm{PhC}$ structure with the underlying $\mathrm{SiO}_{2}$-Toluene effective dielectric region 
Once again, in order to distinguish fluorescence enhancement caused by a photonic bandgap effect from a possible aggregation within the $\mathrm{PhC}$ regions, several structures are fabricated. One of the structures possesses a bandgap surrounding the $1100 \mathrm{~nm}$ emission with r/a ration of 0.33 . A triangular lattice of Si pillars with an r/a ratio of 0.2 is placed adjacent to this structure,. This lattice should have no bandgap. The other two structures below them contained triangular and rhombus lattices of the exact same density as that of the bandgap lattice and a defect region to allow for more quantum dots to be present in the lattice to observe the aggregation effect. The same structure is fabricated on another regular Si sample. This will serve as an asymmetric 'control' representation of the SOI lattices which are expected lack bandgaps no enhancement effect on the fluorescent emission. Figure 5.7 demonstrates SEM images of each of the fabricated lattice within the SOI sample.

When the optical characterization experiment is carried out with the quantum dots still suspended in their toluene solvent (i.e. before evaporation), the $\mathrm{PhC}$ regions with directional bandgap appeared to be brighter than the surrounding. An image of this enhancement is captured along with a co-located image of the fluorescent background was also captured to allow for background noise subtraction. The results are demonstrated in Figure 5.8. The final image after the removal of background noise demonstrated no enhancement for the lattices for which the $1100 \mathrm{~nm}$ emission is not within their bandgaps. By taking 72 random pixels of the $\mathrm{PhC}$ region and another 72 pixels of the background surrounding $\mathrm{PhC}$, the two pixel intensity sets were averaged and then divided by the background level to demonstrate at least 27-fold emission enhancement. Figure 5.11 illustrates the image analysis to observe this enhancement factor.

After 10 minutes, the toluene fully evaporated and all $\mathrm{PhC}$ lattices were observed to be brighter than the surrounding background. This can be attributed to the aggregation of the quantum dots in between the $\mathrm{PhC}$ rods. An image of this aggregation after background removal is shown in Figure 5.10. On a gray scale between 0 and 255, the average pixel intensities of the three different $\mathrm{PhC}$ bright lattices were all around 120. Therefore, this enhancement cannot be attributed to a photonic bandgap effect but due an aggregation effect of having more quantum dots trapped inside the $\mathrm{PhC}$ regions compared to flat surrounding regions, resulting in an apparent, relative increase in emission. This occurs due to the fact that the $\mathrm{PhC}$ lattices all contained pillars that increased the surface area of these regions relative to the surrounding flat regions. Therefore, when the toluene solvent evaporates and the much smaller quantum dots start to adsorb to the Si surface, the PhC regions will have more coverage of the QDs that would result with more emission and a brighter observed gray color. 


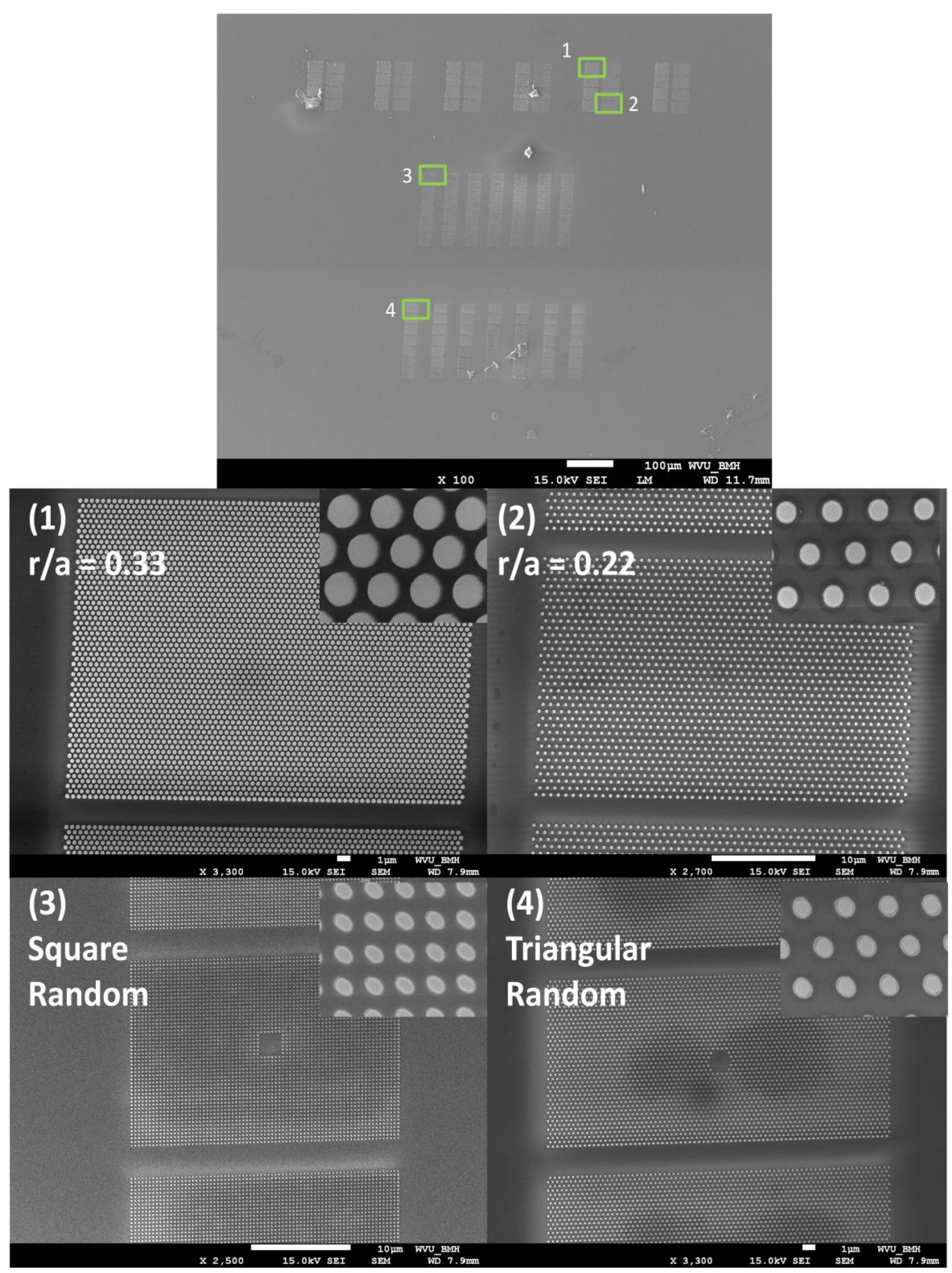

Figure 5.7. SEM images of the fabricated PhC lattices fabricated in one field of view for characterization. (Top) SEM image showing the four different lattices with boxes numbered 1 through 4. (1) SEM image for the structure of box No. 1 with a photonic bandgap surrounding the $1100 \mathrm{~nm}$ emission. (2) Triangular lattice with $\mathrm{r} / \mathrm{a}=0.2$ and no bandgap surrounding the 1100 nm. (3) A random square lattice with no bandgap. (4) A random triangular lattice with no bandgap. Insets are zoomed-in images of each lattice. 


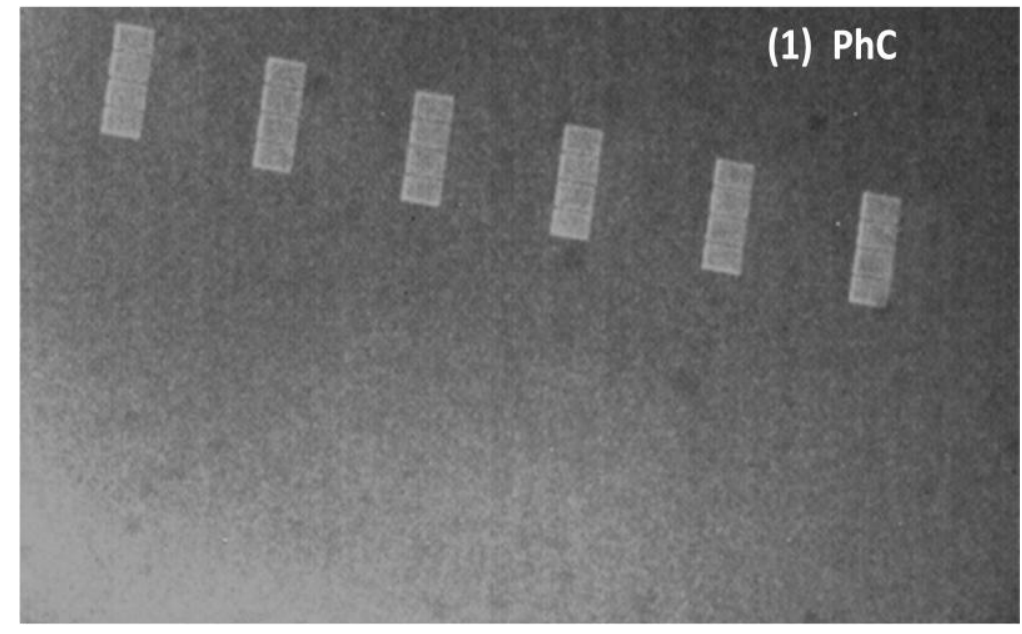

(2) Background
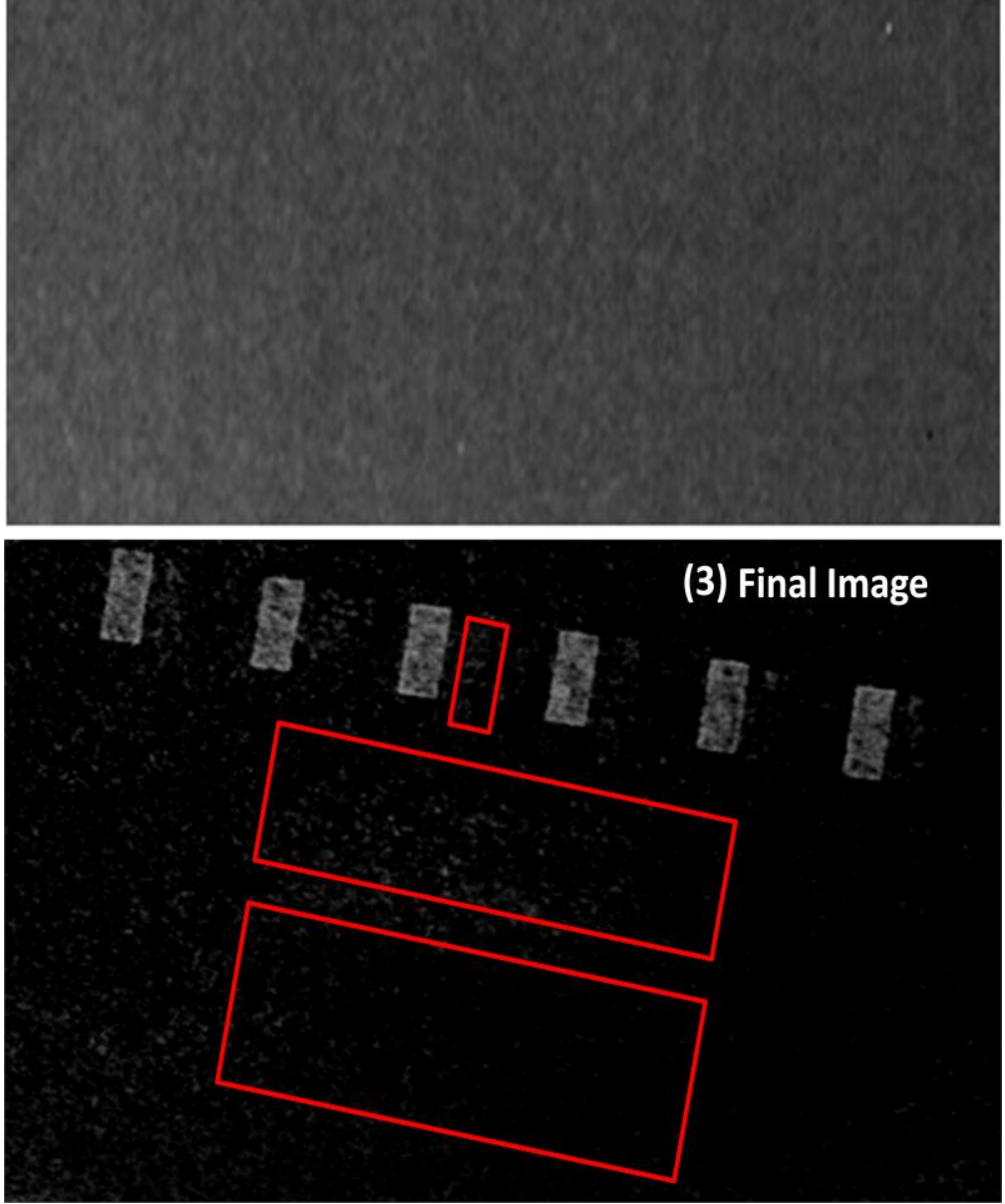

Figure 5.8. Cropped optical image of the (1) PhC lattices region as well as the (2) background and the (3) final image after background subtraction. Red boxes indicate regions where other PhC are fabricated with no bandgaps and hence no enhancement. 

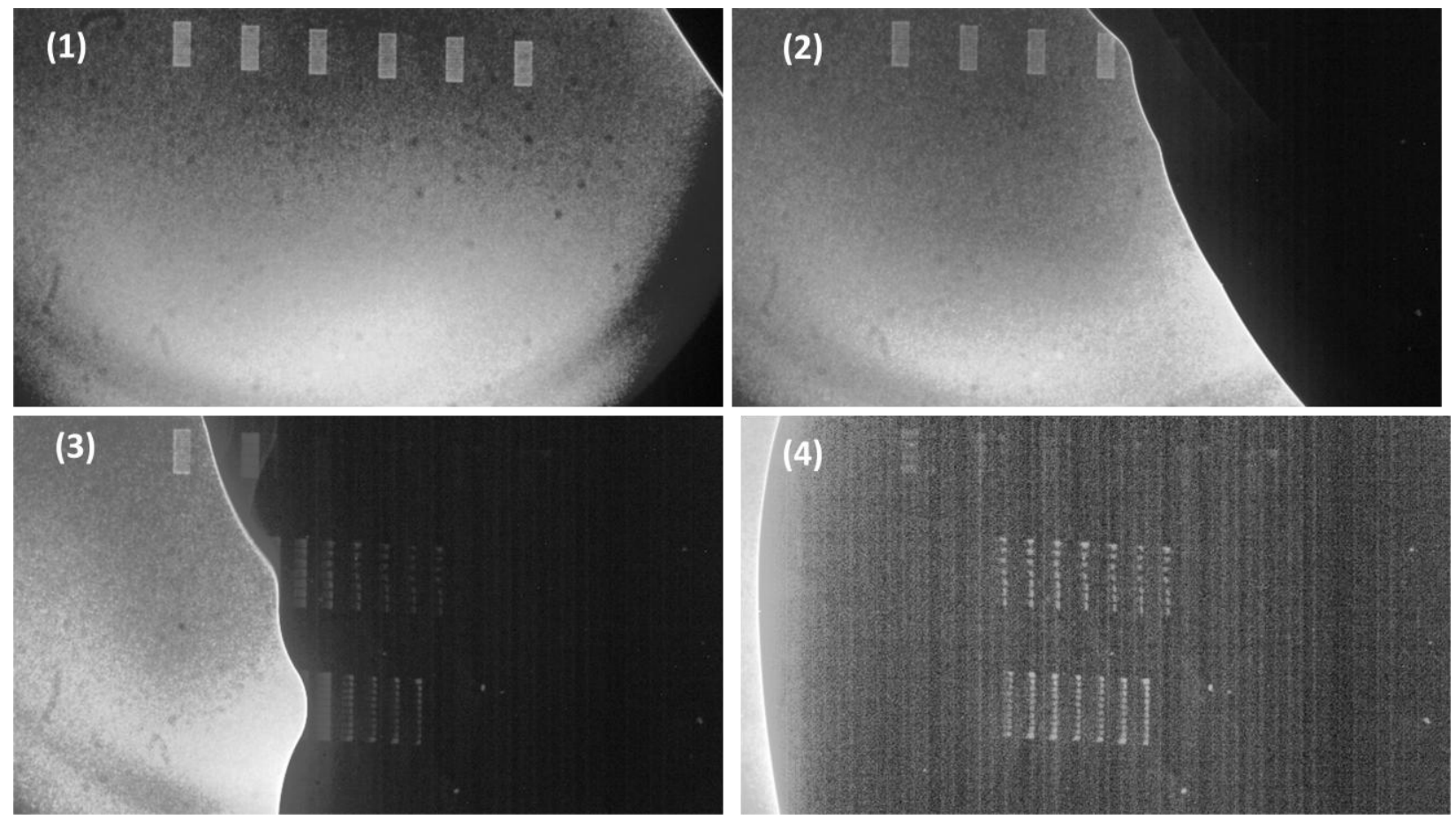

Figure 5.9. Optical images as captured by a Goodrich IR camera of the region containing the $\mathrm{PhC}$ lattices as toluene evaporated. (1) After 30 seconds of placing the sample on the toluene droplet. (2) After 1 minute. (3) After 5 minutes. (4) After 10 minutes

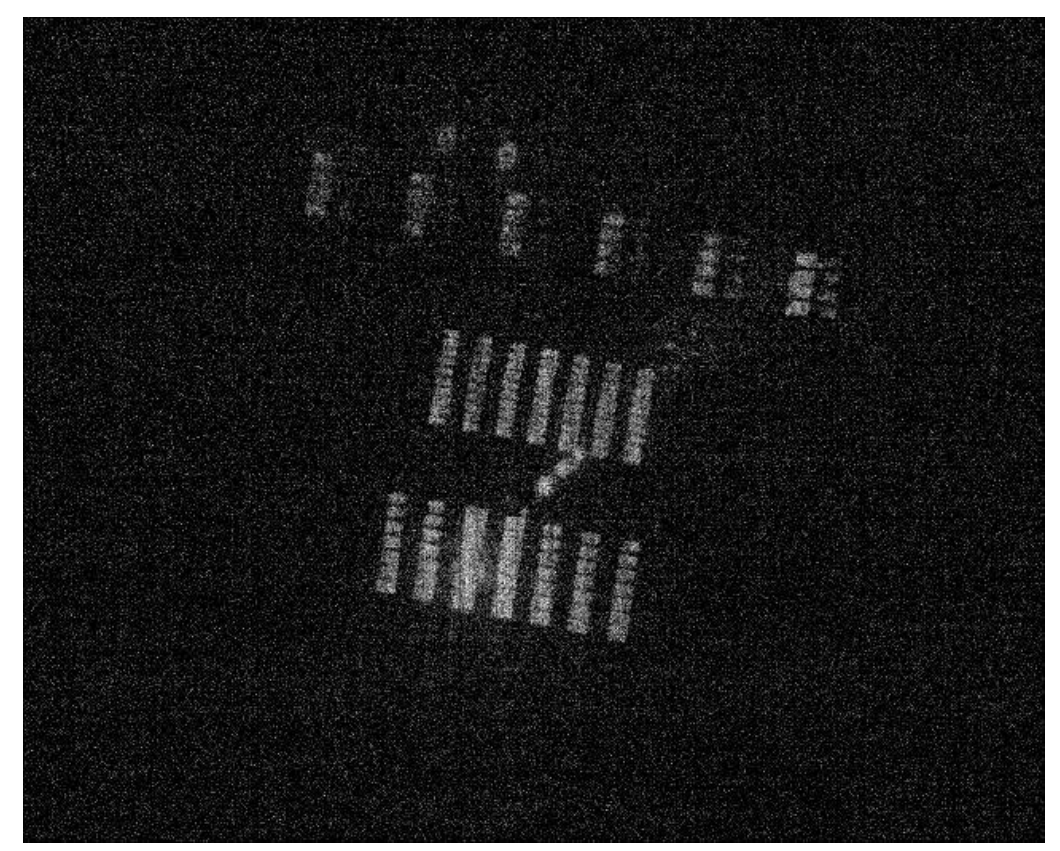

Figure 5.0.10. The different PhC lattice as they appear after the evaporation of the QD solution and after the removal of the background removal. All lattices appear to be brighter due to the aggregation of the QDs within the pillars 


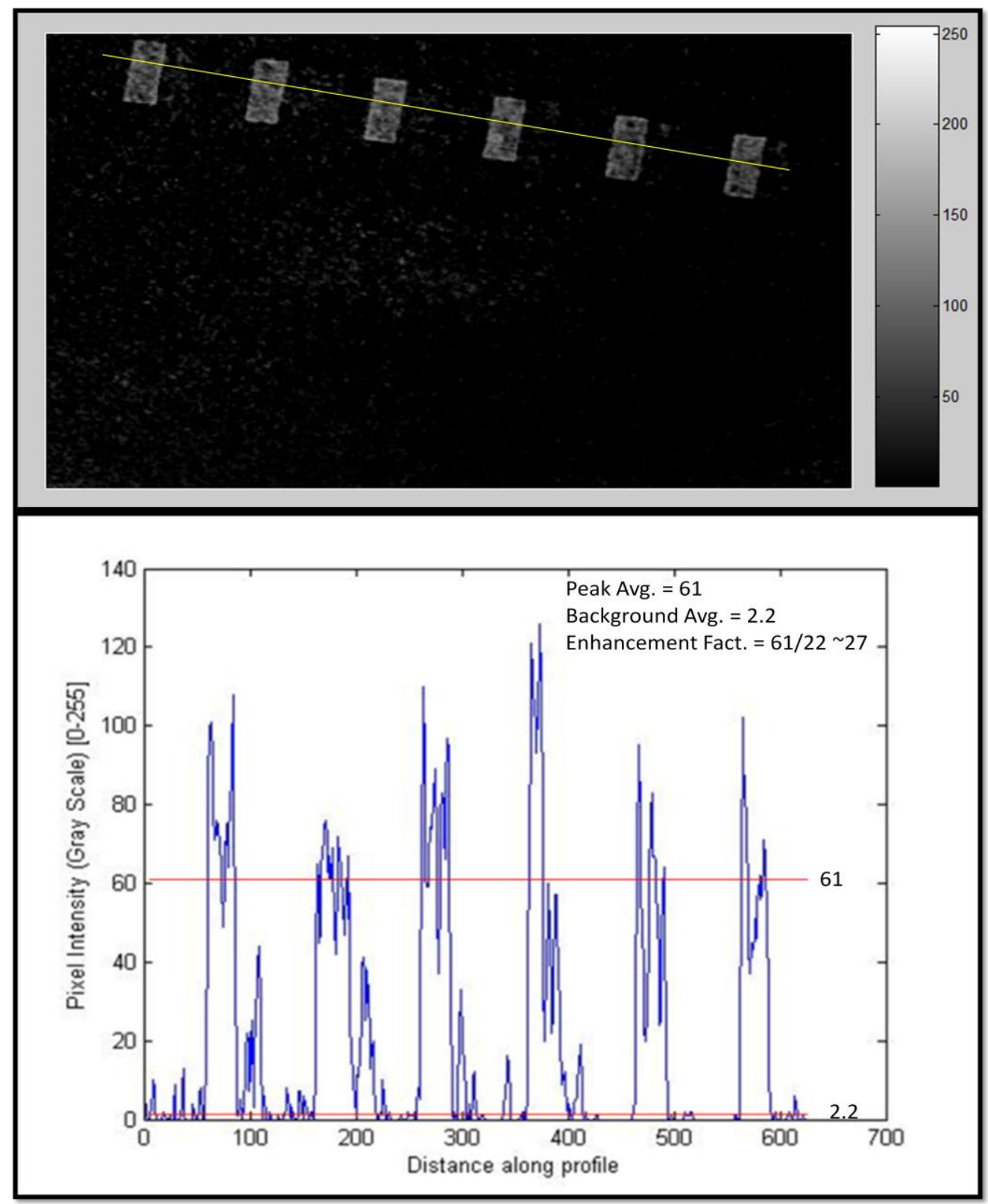

Figure 5.11. (Top) Optical image captured using the Goodrich IR camera with the MATLAB line profile in yellow. (3) Pixel intensity profile as plotted using MATLAB indicating the average peak height of 61 while an average background intensity of 2.2 . 


\section{CHAPTER 6: SUMMARY AND FUTURE DIRECTIONS}

In this work, the unique properties of photonic crystals are utilized to prohibit the in-plane propagation of emitted light from fluorescent molecules for the purpose of achieving an enhancement of this emission by forcing it to propagate outside of the photonic crystal plane where a detector is placed. This occurs due to proper design of the photonic crystal dimensions, focused on placing the bandgap around the emission wavelength of the fluorophore, thereby prohibiting in-plane propagation of this energy.

MIT Photonic Bands (MPB) and MIT Electromagnetic Equation Propagation (MEEP) simulations were performed to gain information regarding bandgap locations and widths, resonant cavity frequencies, and enhancement factors. Unit-less dimensions were extracted in the form of critical dimensions used for device fabrication. The modeling results of silicon, PDMS, and epoxy lattices indicated that wider partial TE or TM bandgaps can observed for triangular photonic crystal lattices of either drilled low-dielectric holes in a high-dielectric slab or high-dielectric rods surrounded by low dielectric background. Finitethickness bandgaps for PDMS and epoxy were not observed due to the low refractive index of these two materials.

Translating the modeling results into actual nano-scale photonic crystal structures was done by following a series of fabrication processes mostly in a cleanroom facility. The fabrication processes of $\mathrm{Si}$ based $\mathrm{PhCs}$ yielded dimensions that deviated from the designed dimensions by very few nanometers. However, the complexity of these processes can significantly increase the final cost of the proposed detection system. Therefore, soft lithographic techniques were also developed and discussed to achieve high-density sub-micron scale photonic crystals in PDMS and epoxy. The molded PDMS PhCs were 130 $\mathrm{nm}$ smaller in diameter than the designed parameters due to possible shrinkage of the molded samples after their release. On the other hand, the molded epoxy PhCs deviated by only $1.14 \%$ from the target dimensions. Optical characterization was done using IR-emitting quantum dots. A 27- fold fluorescent enhancement was observed when characterizing a PhC lattice of silicon pillars surrounded by toluene.

\subsection{FUTURE DIRECTIONS FOR FINITE-THICKNESS SEMICONDUCTOR-BASED PHOTONIC CRYSTALS}

The promising modeling, fabrication, and optical characterization results of finite-thickness slab photonic crystals fabricated in silicon motivate the continuation of the work in this field to integrate these structures with waveguides and biocompatible microfluidic channels and create a portable lab-on-a-chip- 
based detection system. The focus of the presented work has been to demonstrate the ability of photonic crystals to confine and enhance emission that falls within a bandgap. Introducing point defects by altering a single lattice location or a range of points within the lattice will allow for even much higher enhancement factors than what has been achieved in this work. However, using the low-resolution optical setup utilized for this effort can be challenging to characterize such cavities. Instead, Nearfield Scanning Optical Microscopy (NSOM) can be used. The NSPM tip is capable of detecting light generated by quantum dots when they are within the photonic crystal resonant cavities, providing a more accurate characterization of both lattice and defect enhancement.

While a PhC lattice enhancement has been observed based on theoretical bandgap measurements, experimentally characterized photonic bandgaps have not been detected yet. This can be done by first fabricating a $\mathrm{PhC}$ on a regular SOI sample followed by photolithography and etching procedures to align a waveguide surrounding the $\mathrm{PhC}$ region. The waveguide can be designed to have a $90^{\circ}$-bend to prevent the coupled light through the waveguide from affecting the detected signal on other end. A schematic of the structure is shown in Figure 6.1.

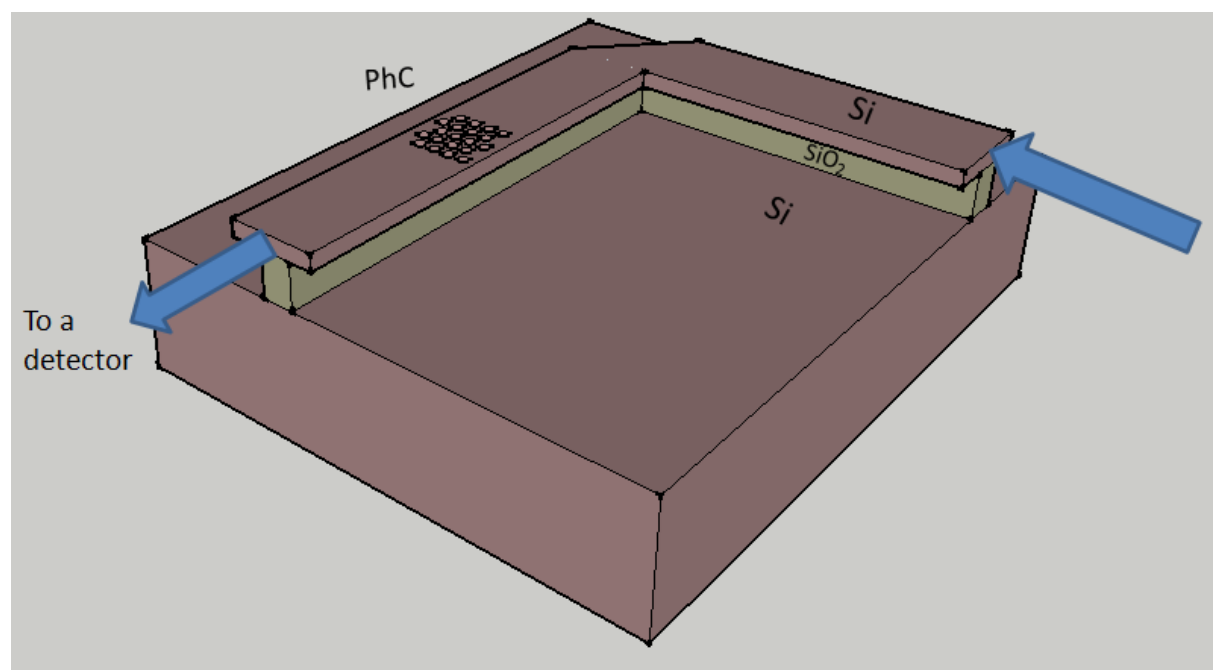

Figure 6.1. The integration of waveguides of photonic crystal structures fabricated in an SOI wafer.

\subsection{FUTURE DIRECTIONS FOR THE POLYMER-BASED PHOTONIC CRYSTALS}

Successful design of photonic crystal lattices with partial photonic bandgaps in PDMS and Epoxy and subsequent development novel nanomolding techniques to fabricate high-density nanometer-scale structures are considered the beginning of the effort to create co-integrated $\mathrm{PhC}$ lattices that are suitable for integrated fluorescence spectroscopy components for portable analysis systems (rapid DNA devices, 
for example). The PDMS and Epoxy materials used in this work are biocompatible and can be easily integrated with microfluidic channels. One major challenge still remain in this area is to design a lattice that is capable of supporting a complete photonic bandgap in finite-thickness slabs made from these materials. This challenge rises from the fact that a slab photonic crystal fabricated in the polymer materials examined in this study will be considered a very small perturbation of the background dielectric constant, making the formation a complete photonic bandgap for modes guided within this slab challenging [49]. A larger study of a wider range biocompatible polymers or mixtures of polymers with higher refractive indices (possibly through the use of doping) is a step forward in this field. This will allow the fabrication of lattices with complete photonic bandgaps, making this class of materials a more viable candidate for $\mathrm{PhC}$ transducer structures in portable sensing platforms. 


\section{BIBLIOGRAPHY}

[1] E. Yablonovitch, "Inhibited Spontaneous Emission in Solid-State Physics and Electronics," Phys. Rev. Lett, 58, 2059-2062 (1987)

[2] S. John, "Strong Localization of Photons in Certain Disordered Dielectric Superlattices," Phys. Rev. Lett., 58, 2486-2489 (1987)

[3] D. W. Prather, S. Shi, A. Sharkawy, J. Murakowski, G. J. Schneider, Photonic Crystals: Theory, Applications, and Fabrication, (John Wiley \& Sons Inc., Hoboken, NJ, 2009)

[4] J. D. Joannopoulos, S. G. Johnson, and J. N. Winn, Robert D. Meade, Photonic Crystals: Molding the Flow of Light (Princeton University Press, Princeton, NJ, 2008).

[5] H. Benisty, V. Berger, J.-M. Gerard, D. Maystre, A. Tchelnokov, Photonic Crystals: Towards Nanoscale Photonic Devices, (Springer, New York, NY 2005)

[6] B. E. A. Saleh and M. C. Teich, Fundamentals of Photonics, (John Wiley \& Sons Inc., Hoboken, NJ, 2007)

[7] V. Bykov, "Spontaneous Emission in a Periodic Structure," Zhurnal Tekhnicheskoi Fiziki, 62 (1972)

[8] V. Bykov, "Spontaneous Emission from a Medium with a Band Spectrum," Sov. J. Quant. Electron., 4, 2059-2062 (1978)

[9] V. Bykov, "Spontaneous Emission in a Periodic Structure," Soviet Physics JETP, 35, 269-273 (1972).

[10] V. Bykov, "Free Oscillations of Elastic Dipole Located in Periodic Structure," Zhurnal Tekhnicheskoi Fiziki, 48 (1978)

[11] S.-Y. Lin and J. G. Fleming, "A Three-Dimensional Optical Photonic Crystal," J. Lightwave Technol., 17, 1944-1947 (1999)

[12] R. V. Nair, R. Vijaya, "Photonic Crystal Sensors: An overview", Journal of Progress in Quantum Electronics, Article in Press

[13] K. Lee and S. A. Asher, "Photonic Crystal Chemical Sensors: pH and Ionic Strength," J. Am. Chem. Soc., 122, 9534-9537 (2000)

[14] A. C. Sharma, T. Jana, R. Kesavamoorthy, L. Shi, M. A. Virji, D. N. Finegold, and Sanford A. Asher, "A General Photonic Crystal Sensing Motif: Creatinine in Bodily Fluids," J. Am. Chem. Soc., 126, 2971-2977 (2004)

[15] Y-J. Lee, S. A. Pruzinsky, and P. V. Braun, "Glucose-Sensitive Inverse Opal Hydrogels: Analysis of Optical Diffraction Response,” Langmuir, 20, 3096-3106 (2004) 
[16] V. L. Alexeev, A. C. Sharma, A. V. Goponenko, S. Das, K. Lednev, C. S. Wilcox, D. N. Finegold, and S. A. Asher, "High Ionic Strength Glucose-Sensing Photonic Crystal," Anal. Chem., 75, 2316-2323 (2003)

[17] Y. Liu, Y. Zhang, and Y. Guan, "New Polymerized Crystalline Colloidal Array for Glucose Sensing," Chem. Commun., 1867-1869 (2009)

[18] R. A. Barry and P. Wiltzius, "Humidity-Sensing Inverse Opal Hydrogels," Langmuir, 22, 1369-1374 (2006).

[19] E. Tian, J. Wang, Y. Zheng, Y. Song, Lei Jiang, and D. Zhu, “Colorful Humidity Sensitive Photonic Crystal Hydrogel," J. Mater. Chem., 18, 1116-1122 (2008).

[20] J. Shi, V. K. S. Hsiao, T. R. Walker, and T. Huang, "Humidity Sensing Based on Nanoporous Polymeric Photonic Crystals," Sensors and Actuators, 129, 391-396 (2008).

[21] T. Sünner, T. Stichel, S.-H. Kwon, T. W. Schlereth, S. Höfling, M. Kamp, and A. Forchel, "Photonic Crystal Cavity Based Gas Sensor," Appl. Phys. Lett., 92, 261112 (2008)

[22] T. Ritari, J. Tuominen, and H. Ludvigsen, "Gas Sensing Using Air-Guiding Photonic Bandgap Fibers," Optics Express, 12, 4080-4087 (2004)

[23] M. Lee and P.M. Fauchet, "Two-dimensional silicon photonic crystal based biosensing platform for protein detection," Optics Express, 15, 4530-4535 (2007).

[24] M. R. Less and P. M. Fauchet, "Nanoscale microcavity sensor for single particle detection," Optics Letters, 32, 3284 (2007)

[25] H. Ouyang, L. A. DeLouise, B. L. Miller, and P. M. Fauchet, "Label-Free Quantitative Detection of Protein Using Macroporous Silicon Photonic Bandgap Biosensors”, Anal. Chem., 79, 1851 (2005)

[26] L. L. Chan, M. F. Pineda, J. Heeres, P. J. Hergenrother, and B. T. Cunningham, "General Method for Discovering Inhibitors of Protein-DNA Interactions using Photonic Crystal Biosensors," ACS Chem. Bio., 3, 437-448 (2008).

[27] N.-N. Aye-Han, Q. Ni, and J. Zhang, "Fluorescent biosensors for real-time tracking of posttranslational modification dynamics," Current Opinion in Chemical Biology, 13 (4), 392-397 (2009)

[28] S. B. VanEngelenburg and A. E. Palmer, "Fluorescent biosensors of protein function," Current Opinion in Chemical Biology, 12 (1), 60-65 (2008)

[29] P. D. Selid, H. Xu, E. M. Collins, M. S. Face-Collins and J. X. Zhao, "Sensing Mercury for Biomedical and Environmental Monitoring," Sensors, 9, 5446-5459 (2009)

[30] Y.-J. Hung, I. I. Smolyaninov, and C. C. Davis, "Fluorescence Enhancement by Surface Gratings," Opt. Exp., 14, 10825 (2006) 
[31] Yuan Yao, Benshun Yi, Jinsheng Xiao, and ZhaoHui Li, "Surface Plasmon Resonance Biosensors and its Application," Bioinformatics and Biomedical Engineering, 2007, ICBBE 2007, July 6-8 2007, pp.1043-1046.

[32] X. D. Hoa, A. G. Kirk, and M. Tabrizian, "Towards integrated and sensitive surface plasmon resonance biosensors: A review of recent progress," Biosensors and Bioelectronics 23, pp. 151-160 (2007).

[33] J. Homola, S. S. Yee, and G. Gauglitz, "Surface plasmon resonance sensors: review," Sensors and Actuators B 54, pp. 3-15 (1999).

[34] M. Skorobogatiy and A. V. Kabashin, "Photon crystal waveguide-based surface plasmon resonance biosensor," Applied Physics Letters 90, 143518 (2006).

[35] K. Aslan, S. N. Malyn, and C. D. Geddes, "Metal-Enhanced Fluorescence from Gold Surfaces: Angular Dependent Emission," J. Fluoresc., 17, 7 (2007)

[36] K. Ray, H. Szmacinski, J. Enderlein, and J. R. Lakowicz, "Distance dependence of surface plasmoncoupled emission observed using Langmuir-Blodgett films,” Appl. Phys. Lett., 90, 251116 (2007)

[37] P. C. Mathias, N. Ganesh, W. Zhang, and B. T. Cunningham, "Graded Wavelength OneDimensional Photonic Crystal Reveals Spectria Characteristics of Enhanced Fluorescence," Jour. Appl. Phys., 103, 094320 (2008)

[38] H.-Y. Wu, W. Zhang, P. C. Mathias, and B. T. Cunningham, "Magnification of Photonic Crystal Fluorescence Enhancement via TM Resonance Excitation and TE Resonance Extraction on a Dielectric Nanorod Surface,” Nanotechnology, 21, 125203 (2010)

[39] J. Nightingale, "Optical Biosensors: Sparrow Biosensor and Photonic Crystal-Based Fluorescence Enhancement," Master's thesis, Lane Dept. of Comp. Sci. and Elec. Engr., West Virginia University, Morgantown, WV, 2008.

[40] S. Yeldandi, "Development of Fabrication Processes for Silicon and Gallium Nitride Photonic Crystal Structures," Master's thesis, Lane Dept. of Comp. Sci. and Elec. Engr., West Virginia University, Morgantown, WV, 2008.

[41] R. Tomkins, "Polarity Inverted GaN for Photonic Crystal Biosensors," Ph.D. dissertation, Dept. of Physics, West Virginia University, Morgantown, WV, 2009

[42] H. Yalamanchili, "Dynamic Bandgap Tuning of Solid Thin Film Photonic Crystal Structures," Master's thesis, Lane Dept. of Comp. Sci. and Elec. Engr., West Virginia University, Morgantown, WV, 2010

[43] H. Andagana, "Nanofabrication of Optofluidic Photonic Crystal Resonators for Bioesnsing," Master's thesis, Lane Dept. of Comp. Sci. and Elec. Engr., West Virginia University, Morgantown, WV, 2010 
[44] J. M. Dawson, J. R. Nightingale, R. P. Tompkins, X. Cao, T. H. Myers, L. A. Hornak, and D. Korakakis, “GaN Photonic Crystal-Based, Enhanced Fluorescence Biomolecule Detection System,” Proc. Materials Research Society, 1040 (2008)

[45] B. M. Hamza, H. Yalamanchili, H. Andagana, X. Cao, L. A. Hornak1, J. M. Dawson, and D. Korakakis, "Top-Down Approach to the Fabrication of GaN-based Photonic Crystal Biosensor," Proc. Materials Research Society, 1133 (2008)

[46] B. M. Hamza, A. Kadiyala, C. Kilemi, Y. Liu, and J. M. Dawson, "Fluorescence Enhancement in a Polymer-based Photonic Crystal Biosensor,” Proc. SPIE, 7888, 788804 (2011)

[47] S. G. Johnson, "Photonic Crystals: From Theory to Practive," PhD Dissertation, Department of Physics, Massachusetts Institute of Technology, Cambridge, MA (2001)

[48] S. G. Johnson, P. R. Villeneuve, S. Fan, and J. D. Joannopoulos, "Linear Waveguides in PhotonicCrystal Slabs," Phys. Rev. B, 62, 12 (2000)

[49] S. G. Johnson, S. Fan, P. R. Villeneuve, and J.D. Joannopoulos, "Guided Modes in Photonic Crystal Slabs," Phys. Rev. B, 60, 8 (1999)

[50] S. G. Johnson and J. D. Joannopoulos, The MIT Photonic-Bands Package (http://abinitio.mit.edu/mpb/)

[51] S. G. Johnson and J. D. Joannopoulos, The MIT Electromagnetic Equation Propagation Package (http://ab-initio.mit.edu/meep/)

[52] M. J. Madou, Fundamentals of Microfabrication, (CRC Press, Boca Raton, FL, 2002)

[53] S. Chakraborty , Microfluidics and Microfabrication, (Springer, New York, NY, 2010)

[54] M. J. Jackson, Micro and Nanomanufacturing, (Springer, New York, NY 2007)

[55] J. P. Ballantyne, G. R. Brewer, Electron-beam Technology in Microelectronic Fabrication, (Academic Press, New York, NY 1980)

[56] S. Seal, Functional Nanostructures: Processing, Characterization, and Applications, (Springer, New York, NY 2008)

[57] Z. Cui, Micro-nanofabrication: Technologies and Applications, (Springer, New York, NY, 2005)

[58] C. A. Bishop, Vacuum Deposition onto Webs, Films, and Foils, (William Andrew Inc., Norwich, NY, 2007)

[59] EECpE Research Lab at the University of Texas in El Paso (http://wwwold.ece.utep.edu/research /cdte/Fabrication/index.htm)

[60] M. Sugawara and B. L. Stansfield, Plasma Etching: Fundamentals and Applications, (Oxford University Press, New York, NY, 2002)

[61] Matthieu Lagouge - Microtechnology (Dry Etching) (http://matthieu.lagouge.free.fr/ $\underline{\text { microtechnology/dry etch.html) }}$ 
[62] R. Doering and Y. Nishi, Handbook of Semiconductor Manufacturing Technology, (CRC Press, Boca Raton, FL, 2008)

[63] Nanometer Pattern Generation System by JC Nabity (http://www.jcnabity.com/overview.htm)

[64] MicroResist Technology ma-N 2400 series resist (http://www.microresist.de/products/ negative_photoresists/ma_n_2400_en.htm)

[65] J. K. Yang, "Fabrication of Superconducting Nanowire Single Photon Detectors," Master's Thesis, Department of Electrical Engineering and Computer Science, Massachusetts Institute of Technology, Cambridge, MA, 2005.

[66] N. Gadegaard and D. McCloy, "Direct Stamp Fabrication for NIL and Hot Embossing using HSQ," Microelectronics Engineering, 84, 2785-2789 (2007)

[67] S. Choi, M. Yan, and I. Adesida, "Fabrication of Triangular Nanochannels using the Collapse of Hydrogen Sisesquioxane Resists," App. Phys. Lett., 93, 163113 (2008)

[68] K. A. Lister, B. G. Casey, P. S. Dobson, S. Thomas, D. S. Macintyre, C. D. W. Wilinson, and J. M. R. Weaver, "Pattern Transfer of a 23 nm-Period Grating and Sub-15 nm Dot into CVD Diomond," Microelectronics Engineering, 73-74, 319-322 (2004)

[69] V. Passi, A. Lecestre, C. Krzeminsk, G. Larrieu, E. Dubois, J.-P. Raskin, “A Single Layer Hydrogen Sisesquixane (HSQ) Based Lift-Off Process for Germanium and Platinum," Microelectronics Engineering, 87, 1872-1878 (2010)

[70] S. Lis, R. Dylewicz, J. Mysliwiec, A. Miniewicz, S. Patela, "Application of Flowable Oxides in Photonics," Materials Science-Poland, 26, 1 (2008)

[71] A. G. Caster, S. Kowarik, A. M. Schwarzberg, O. Nicolet, S.-H. Lim, and S. R. Leone, “Observing Hydrogen Sisesquioxane Cross-Linking with Broadband CARS,” J. Raman Spectrosc., (2009)

[72] Y. Xia and G. M. Whitesides, "Soft Lithography," Annu. Rev. Mater. Sci., 28, 153-184 (1998)

[73] R. Bashir, "BioMEMS: State-of-the-art in Detection, Opportunities and Prospects," Advanced Drug Delivery Reviews, 56, 1565-1586 (2004)

[74] D. B. Weibel, W. R. Diluzio, and G. M. Whitesides, "Microfabrication Meets Microbiology," Nature Reviews Microbiology, 5, 209-218 (2007)

[75] T. V.-Dinh and B. Cullum, "Biosensors and Biochips: Advances in Biological and Medical Diagnostics," Fresenius J Anal Chem, 366, 540-551 (2000)

[76] E.M. Purcell, "Spontaneous emission probabilities at radio frequencies," PhysicsReview Letters, 69, 681 (1946)

[77] D. Kleppner, “Inhibited Spontaneous Emission,” Physics Review Letters, 47, 233-236 (1981).

[78] Jurgen Fritz, “Cantilever biosensors,” Analyst, 133, 855-863 (2008). 
[79] B. J. Privett, J. H. Shin, and M. H. Schoenfisch, "Electrochemical Sensors," Analytical Chemistry, 80, 4499-4517 (2008).

[80] S. M. Borisov and O. S. Wolfbeis, “Optical Biosensors," Chemical Reviews, 108, 423-461 (2008). 


\section{APPENDIX A: E-BEAM LITHOGRAPHY CAD FILE PROCESS}

When the lattice constant and the radius of a triangular photonic crystal lattice are known, a DesignCAD file can be created. In this section, a generic step by step procedure adapted and modified from [43] will be demonstrated to create a simple triangular lattice DesignCAD file that can be used to create a run file and therefore fabricate a PhC structure of either holes in PMMA or pillars of ma-N, depending on the desired final features and the type of resist that is used. Descriptive images of some the steps are explained after the step.

1. Open the NPGS software by double clicking on the NPGS green icon on the Desktop of the central computer

2. Select your project from the 'Current Project Directory' drop-down menu

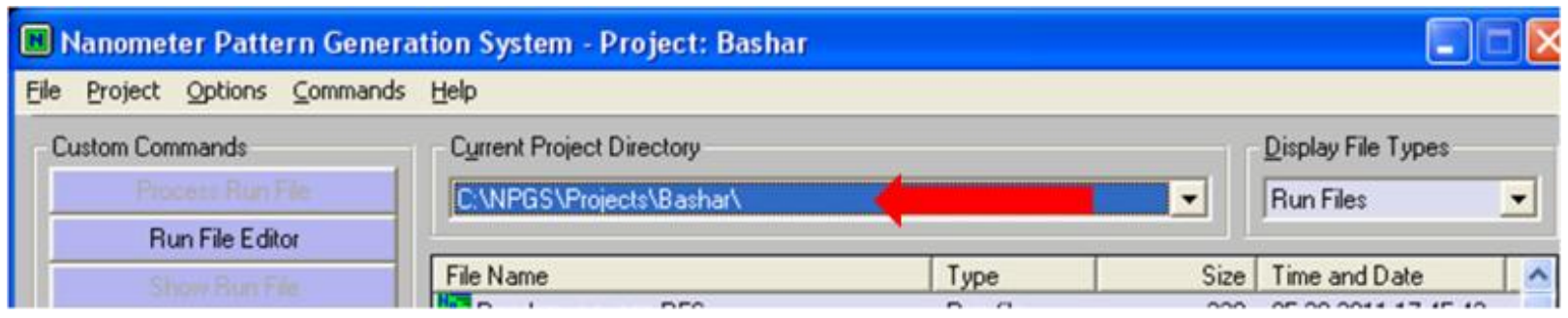

Figure A-1. Project selection on NPGS. Red arrow indicating the current project directory.

3. On the left side of the Custom Commands list, click on 'DesignCAD Express' to start creating the CAD file. Accept the initialization menu when DesignCAD Express tool opens up by hitting any key.

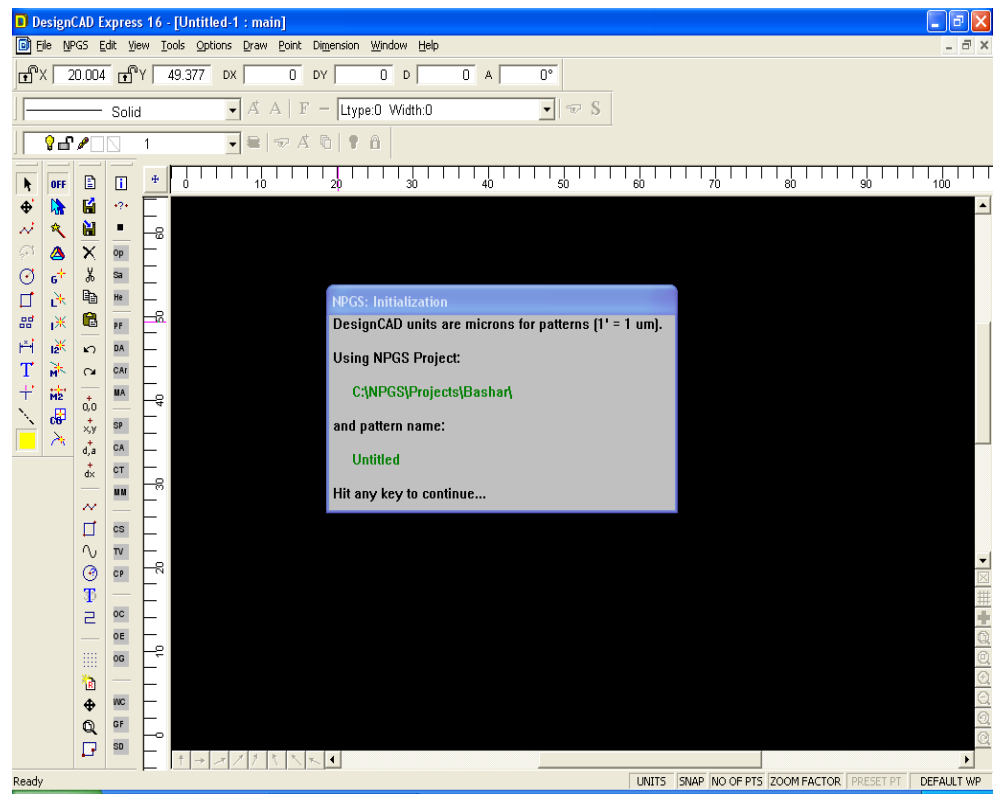

Figure A-0.2. A screenshot of the DesignCAD software 
4. To start creating the PhC lattice, a line indicating the lattice constant needs to be drawn first. To do so, from the 'Draw' menu in the top, select 'Lines' and then 'Ortho Line'.

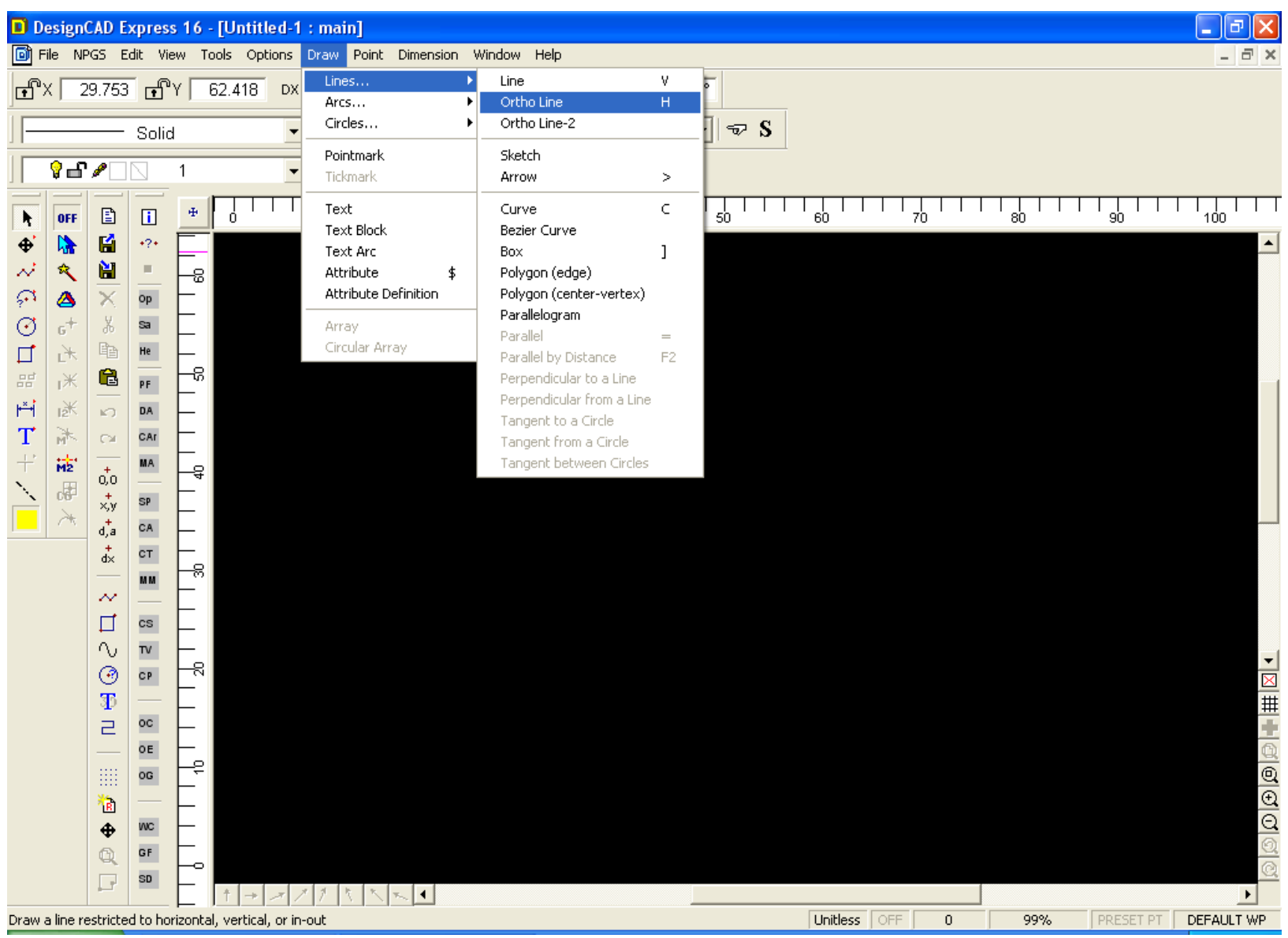

Figure A-3. Drawing a horizontal line process 
5. The cursor shape will then change to a + sign. Click anywhere in the back region of the CAD tool only once and then move the mouse to right. On the keyboard, hit " key (to the left of Enter). 'Point Relative" box will show up to allow you to type a lattice constant value in micrometer units. Click OK once a value is typed and then hit Enter to allow the value to be applied to the drawn line.
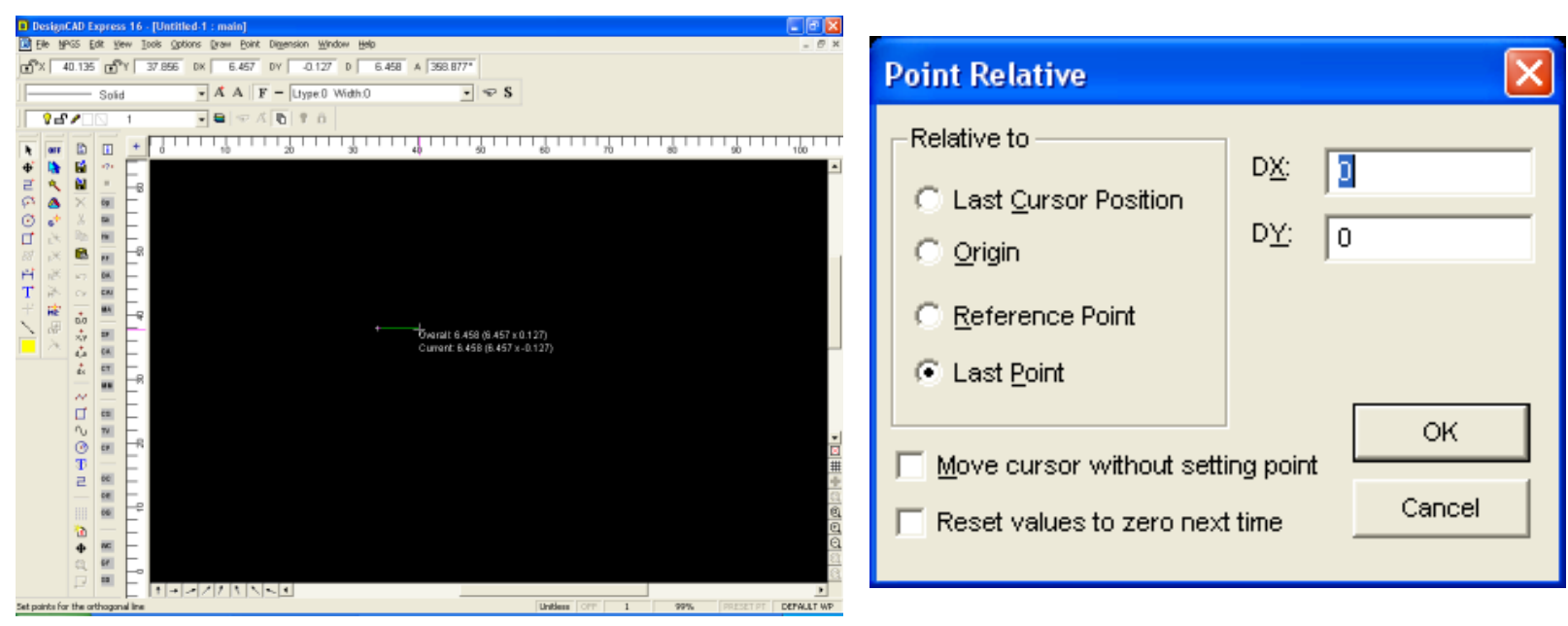

Figure A-4. Drawing a horizontal line and giving its length which is normally the lattice constant when design PhC patterns.

6. To zoom in, Ctrl + mouse scroll wheel can be used. The line that is just drawn will be simply one side of a hexagon which will contain circles at each corner and then replicated in the $\mathrm{x}$ and $\mathrm{y}$ directions to create the complete lattice. To draw the hexagon, click on Draw $\rightarrow$ Lines $\rightarrow$ Polygon (Edge) and type 6 to be the number of sides of the polygon.

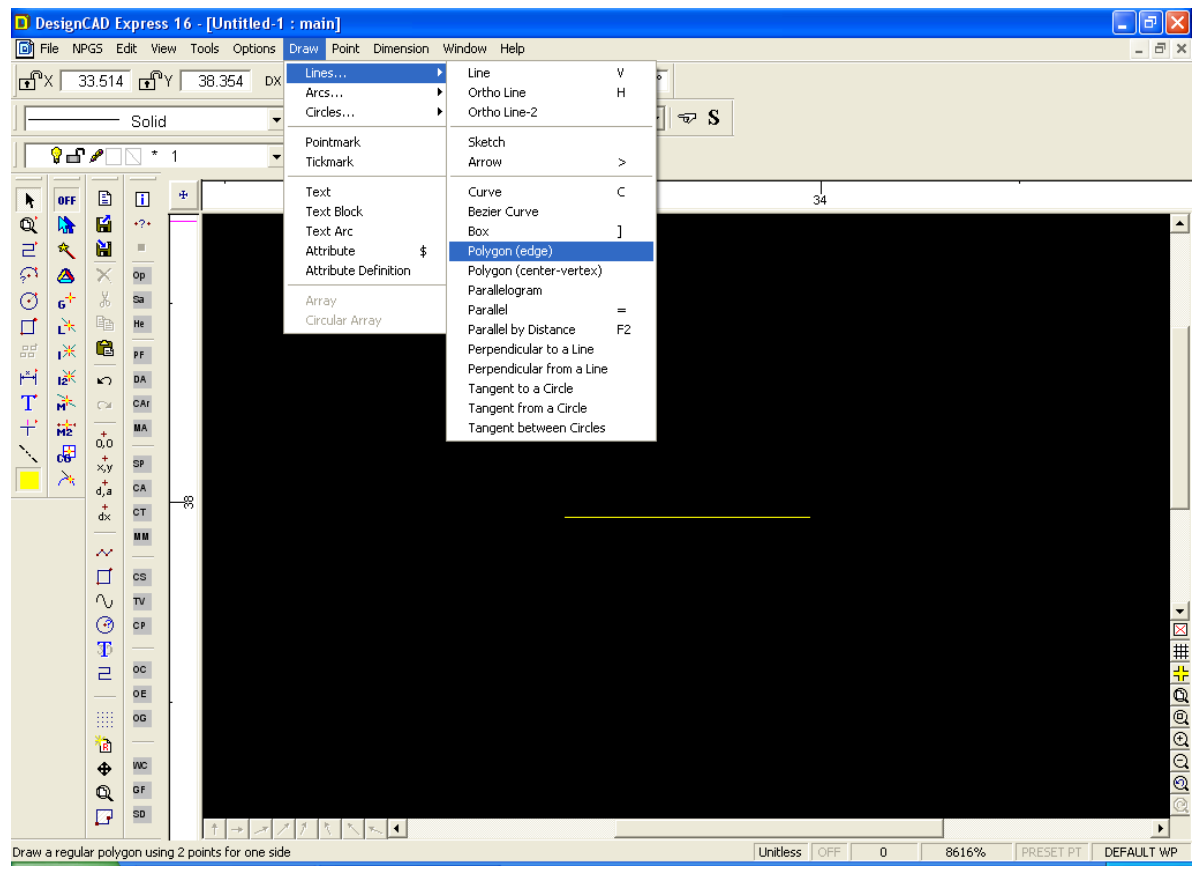

Figure A-0.5. Drawing a polygon procedure 
7. Right click next to one end of the line and once again next to the other end of the line to place one side of the hexagon on the lattice constant line. Click once again on that side and hit delete to remove the underlying original lattice constant line.

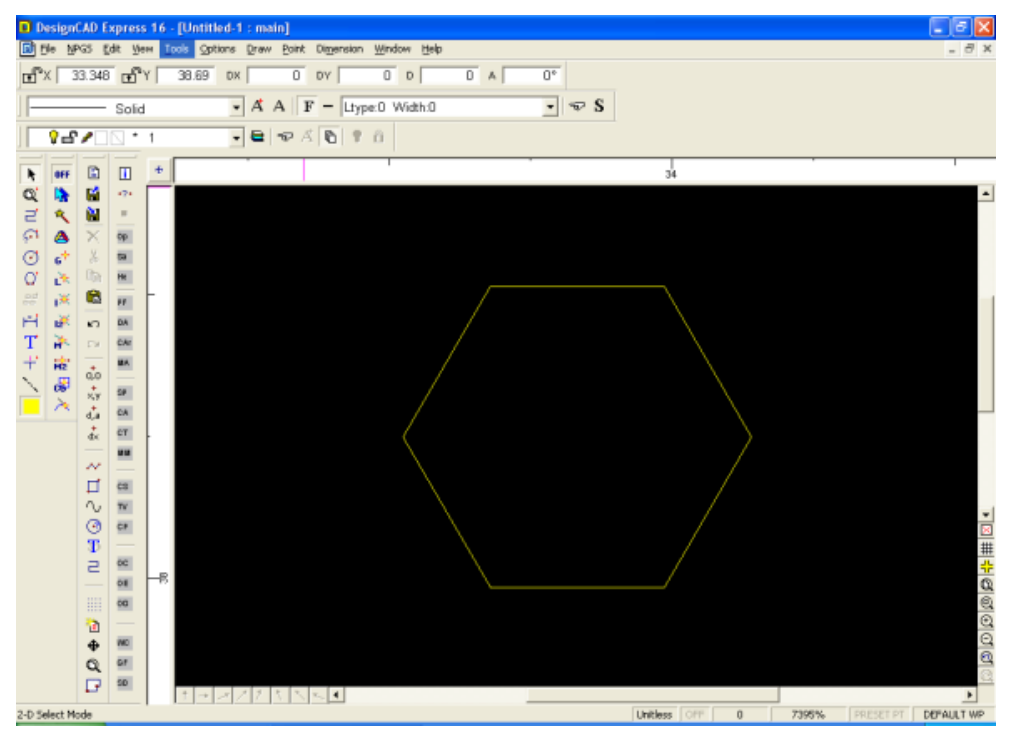

Figure A-0.6. The polygon shape at the end of steps 6 and 7

8. To draw a circle on a corner of the hexagon, Click on Draw $\rightarrow$ Circles $\rightarrow$ Circle (center, outside)

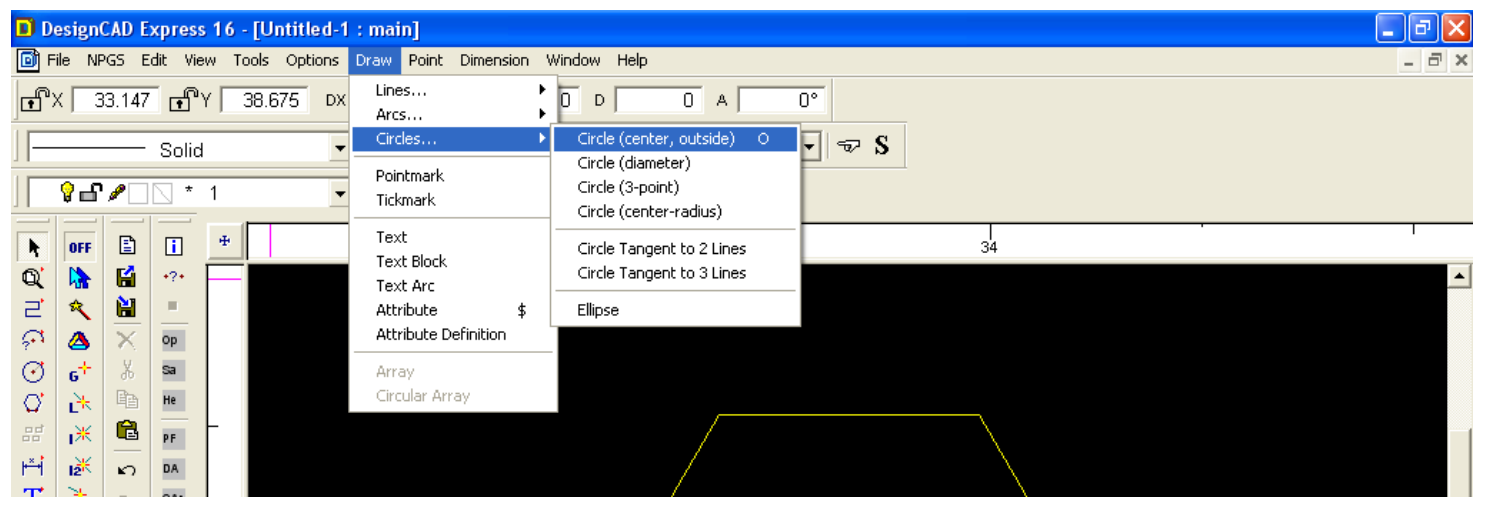

Figure A-7. Circle drawing steps

9. Right click once next to the top left corner of the hexagon to place the center of the circle at that edge and drag the mouse outside and click to define the circle. To change the circle radius and the exposure type of this circle, hit Ctrl+i to open the 'Cir Arc' box. Change the radius to the desired value in micrometer units. Also change the default line type from 'Solid' to 'Dashed'. Close the dialog box once done 


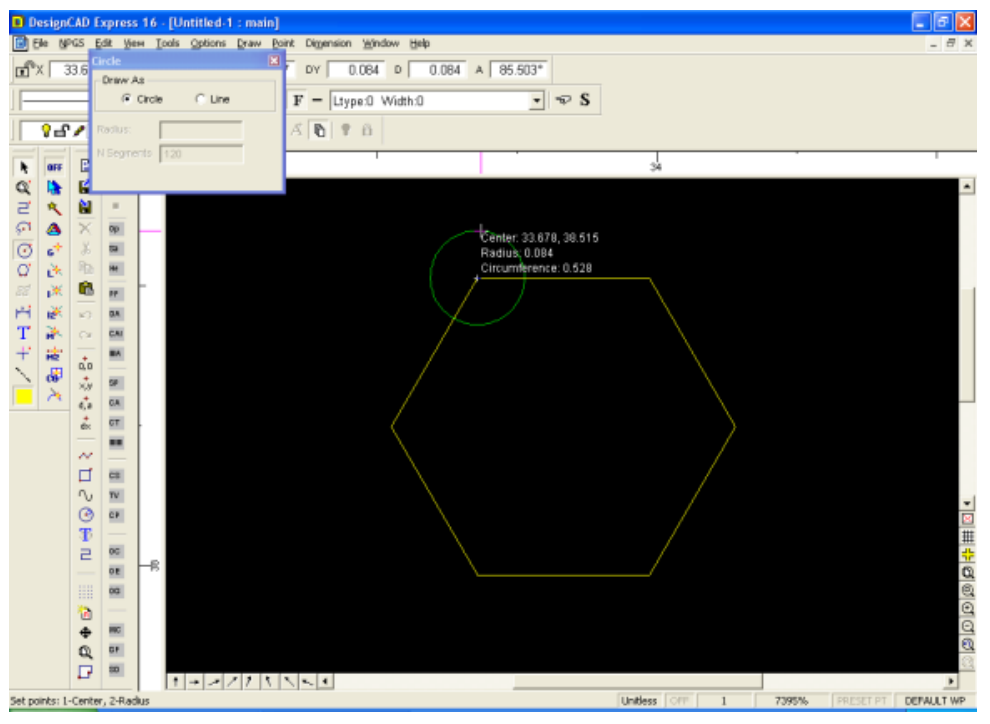

\begin{tabular}{|c|c|}
\hline D Cir Arc & $x$ \\
\hline 8ロー & \\
\hline$\vec{\nabla} \geqslant 0$ और & \\
\hline Name: Circle & $\Delta$ \\
\hline Center Coordinate & \\
\hline$\underline{x} \quad 33.6777$ & \\
\hline$\underline{Y}: \quad 38.5147$ & \\
\hline Radius: $\quad$ & \\
\hline Start Angle: 50.9008 & \\
\hline Span Angle: 360 & \\
\hline Length: $\quad 0.4867$ & \\
\hline$\Gamma$ Lock Radius & \\
\hline Bounding Box- & \\
\hline Wiath: $\longdiv { 0 . 1 5 4 9 }$ & \\
\hline Height: 0.1549 & \\
\hline$\Gamma$ Use Select Handle & \\
\hline - solid & \\
\hline Line Șcale: $\quad 0.2$ & \\
\hline Line Width: 0 & \\
\hline V Eill Wide Line & \\
\hline$-0.00 \mathrm{~mm}=$ & \\
\hline$\Gamma$ Enable Fixed Lineweight & \\
\hline$\Gamma$ Color by Layer & \\
\hline$\ulcorner$ Line Style by Layer & -1 \\
\hline
\end{tabular}

Figure A-8. Identifying the circle radius and line type

10. To create an array of the circle, click on the icon 'MA' and click once below the left bottom edge of the circle and again above the top right side of the circle to enclose the whole circle by the selection.
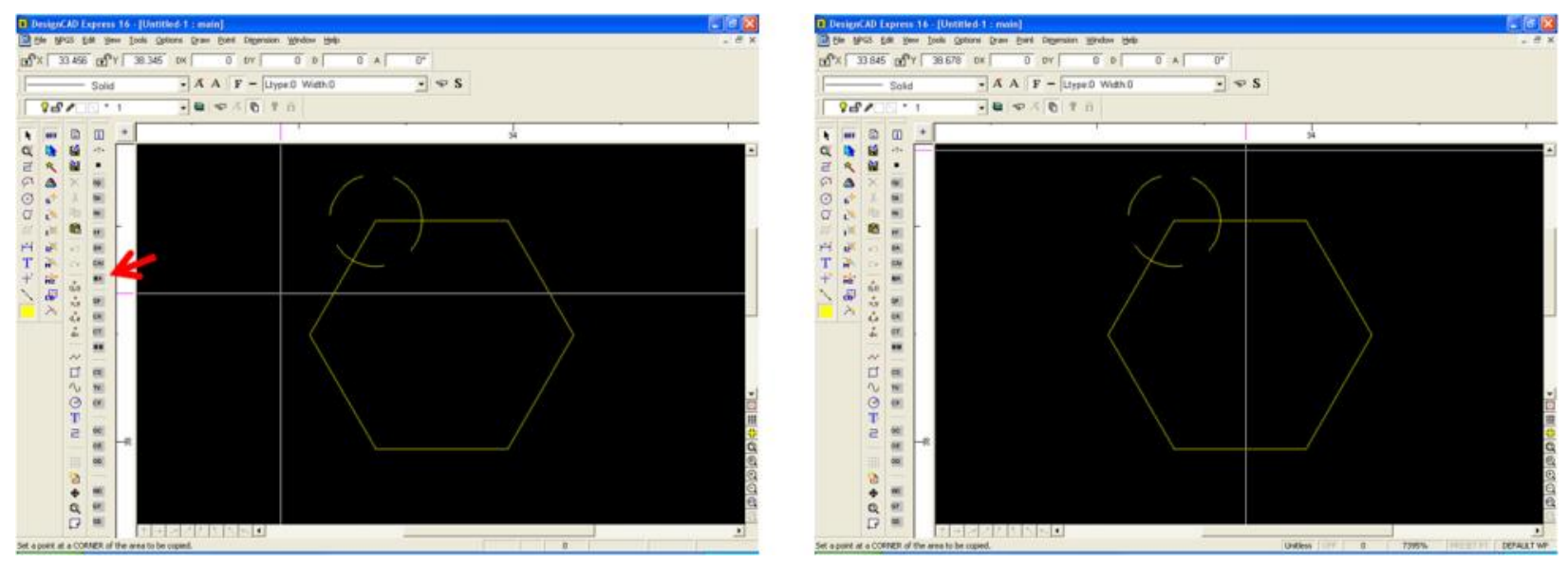

Figure A-9. 'Make Array' procedure. Red arrow indicates where the MA icon can be found. Left image indicates the first click below the bottom left corner of the feature and the right image indicates the second click above the top right corner of the feature 
11. A series of dialog boxes will then pop out. At this step, the first row of the photonic crystal can be created. Therefore, in the first box regarding the number of columns, enter the desired number periods in the $\mathrm{x}$ direction (75 for instance) and then click OK.

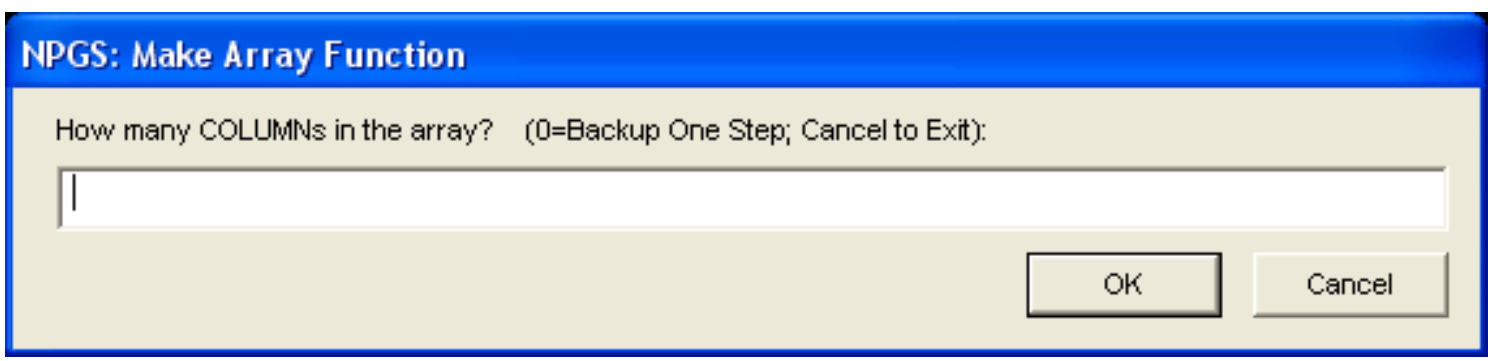

Figure A-10. Entering the number of columns of the desired array

12. In the second dialog box, enter the spacing between the columns, which is the lattice constant. Click $\mathrm{OK}$ and then $\mathrm{N}$ if an overlapping warning shows up.

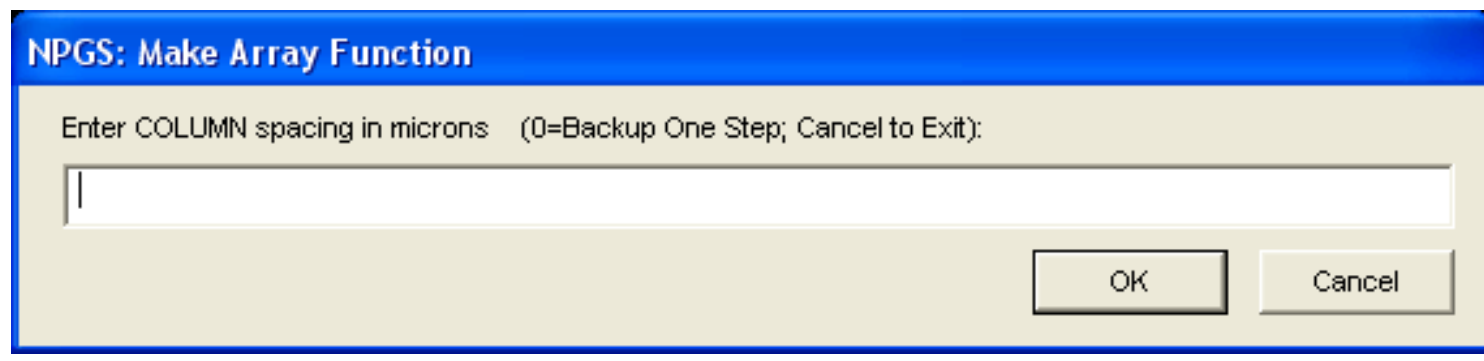

Figure A-11. Entering the desired column spacing of the array

13. Since at this step, we just want to create the first row, type 1 for the number of row. Click on OK.

14. To start creating the second row circles, repeat steps 6 through 13 but by create a circle on the left corner of the hexagon this time.

15. Once the first two rows are created, the MA command can be used to replicate the rows and create the whole lattice by creating copies of these two rows in the y direction. To do so, use the ? icon to measure the distance between top left corner and bottom left corner of the hexagon. This value will be used in the next step.

16. Delete the hexagon and then click on MA and enclose the first two rows. 


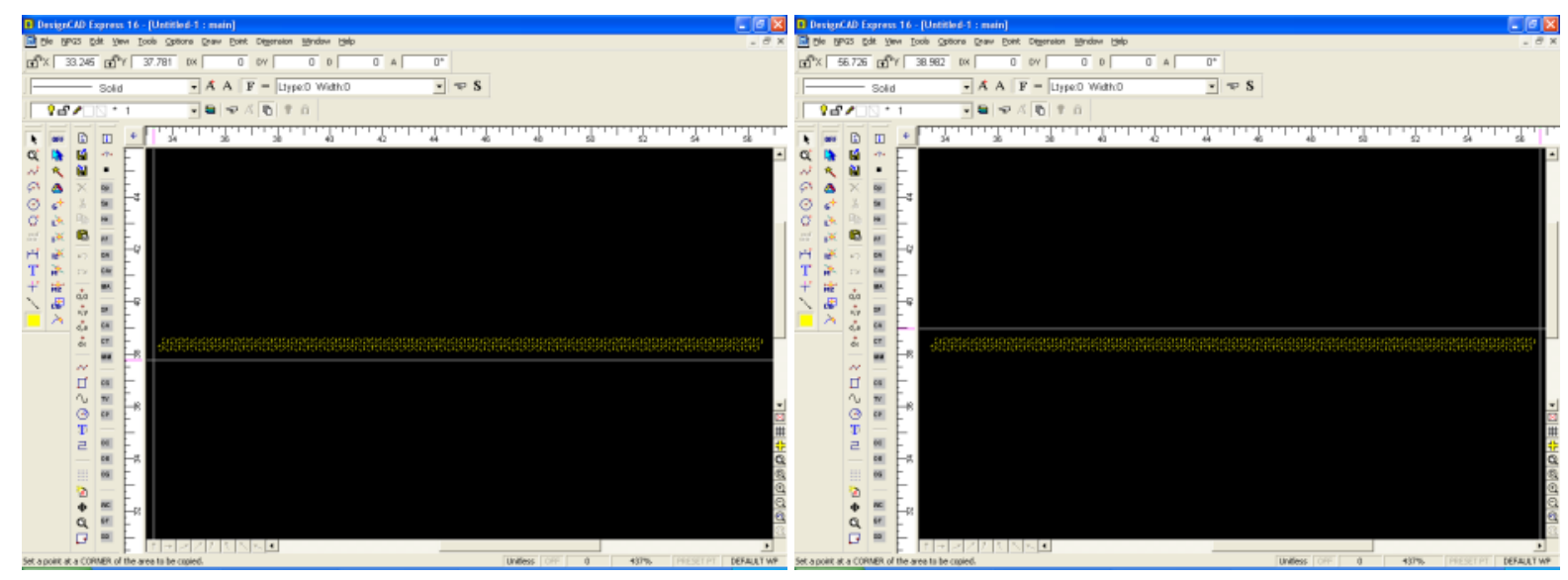

Figure A-12. Making an array of the first two rows of the triangular lattice of circles.

17. To create this array, type 1 for the number of columns and then type a desired number of the periods in the $\mathrm{Y}$ direction (75 for instance) and the hit $\mathrm{N}$ when an overlapping warning shows up. Continue hitting Enter until the pattern is replicated in the Y direction. Reject the color or layer assignment dialog boxes.

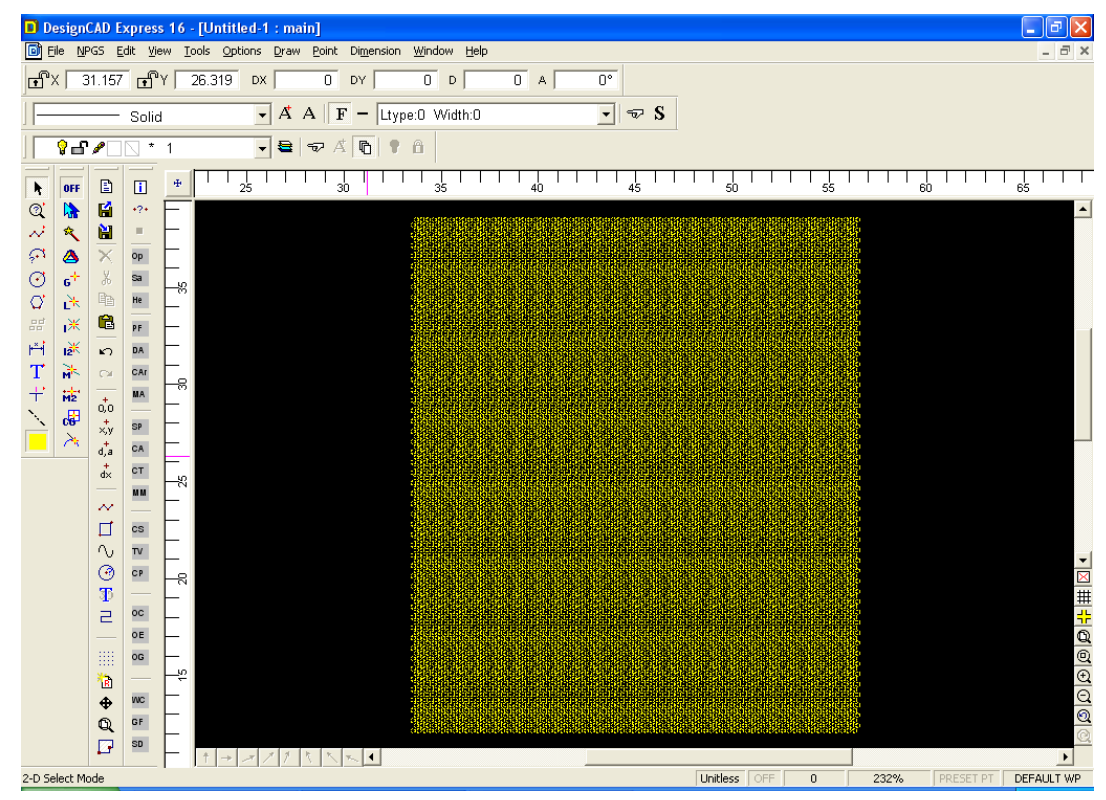

Figure A-13. One block of a triangular $\mathrm{PhC}$ lattice

18. In order to replicate the $\mathrm{PhC}$ region replicated in the $\mathrm{X}$ or $\mathrm{Y}$ directions, use the MA method again and accept the automatic color assignment upon completion by hitting $\mathrm{y}$. 


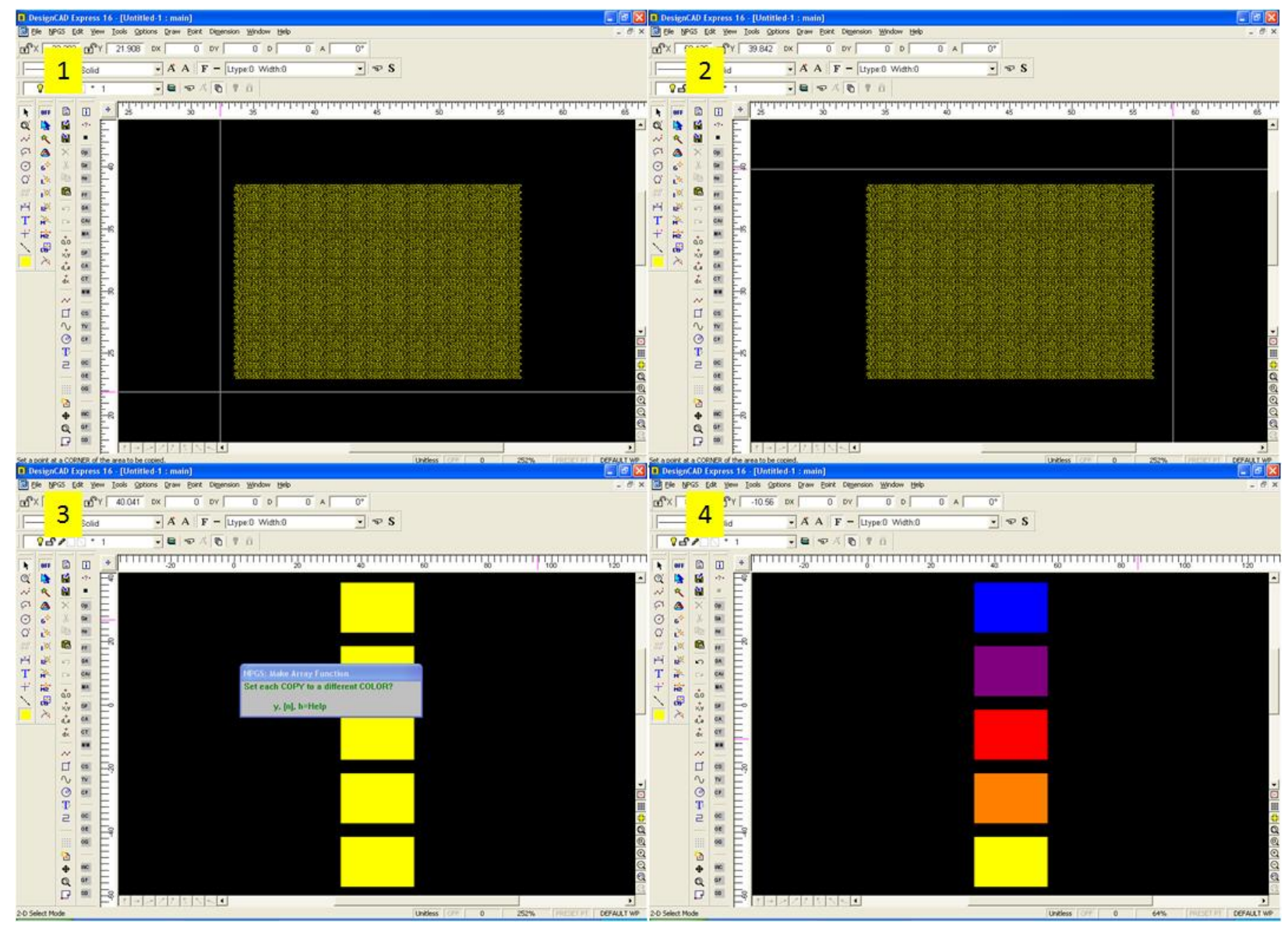

Figure A-14. Making an array of the PhC block for different doses.

19. At the end, save the file by clicking on NPGS $\rightarrow$ Save file and give it a desired name.

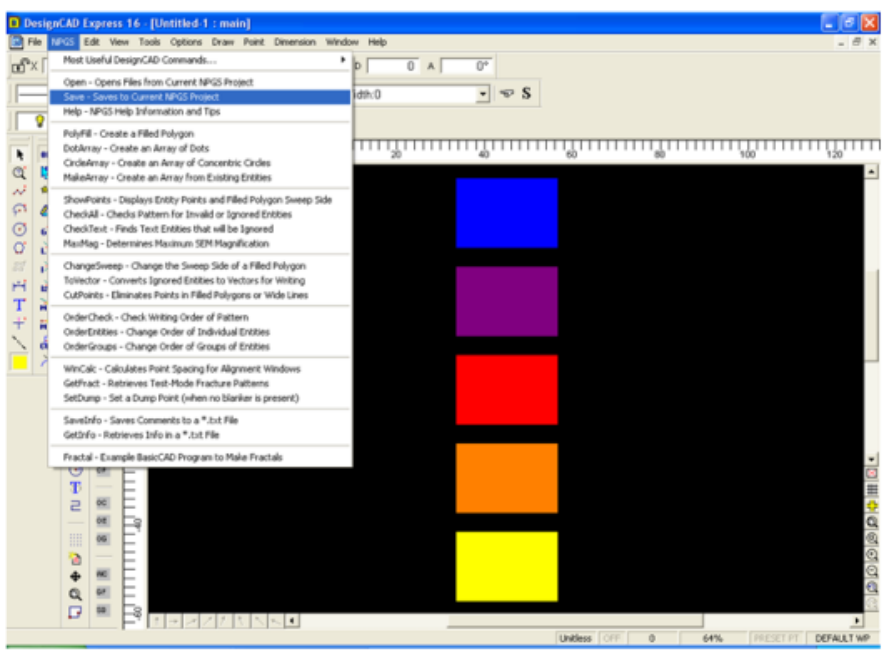

\begin{tabular}{|c|c|c|c|c|}
\hline \multicolumn{4}{|l|}{ 国 File Save } & $\bar{x}$ \\
\hline File Name & Type & Size & Time and Date & $\hat{\imath}$ \\
\hline DOPDMS_1130nm_a4... & DesignCAD ... & $69451 \ldots$ & $2 / 28 / 2011$ 11:02:25. & \\
\hline DOPDMS_1150 nm_a5... & DesignCAD ... & $16705 \ldots$ & 10/3/20109.51:08 PM & \\
\hline QPOPOS_I300nm_a1... & DesignCAD ... & 723445 & 10/3/2010 7:51:28 PM & \\
\hline QPOPMS_varied_a.dc2 & DesignCAD ... & 813526 & $1 / 10 / 201111: 09: 47 \ldots$ & \\
\hline Q⿱一𫝀口) Random_no_bandg. & DesignCAD ... & $16634 \ldots$ & 5/30/2011 5:27:56 PM & \\
\hline${ }_{\triangle}^{\circ}$ Rods_Si_4_18_201... & DesignCAD ... & $36499 .$. & $4 / 18 / 201110: 25: 16 \ldots$ & \\
\hline$\triangle^{\circ}$ Rods_Si_quare_4_... & DesignCAD ... & $63547 \ldots$ & $4 / 18 / 2011$ 10.53.21 ... & \\
\hline$\Delta^{\circ}$ rohizdc2 & DesignCAD ... & 3143 & $12 / 14 / 201012: 00 ; 46 \ldots$ & \\
\hline$\triangle^{\circ}$ small_PhC_with_H1... & DesignCAD ... & $128345 \ldots$ & 10/17/20109:10:48 ... & \\
\hline$\square^{\circ}{ }^{\circ}$ SOl_enlargedH1.dc2 & DesignCAD ... & $18099 .$. & 12/9/2010 1:44:12 AM & \\
\hline D०SOl_full_bgap.dc2 & DesignCAD ... & $13786 .$. & 11/27/20101:45:33 ... & \\
\hline 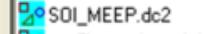 & DesignCAD ... & $11328 \ldots$ & 11/3/2010 10:30:08 ... & \\
\hline${ }_{40}^{\circ}$ sunflower_Anand.d. & DesignCAD ... & 82692 & 9/9/2010 3:47:46 PM & \\
\hline${ }_{\triangle}^{\circ}$ SSUNFLOWER1.dc2 & DesignCAD ... & $64545 \ldots$ & 9/9/2010 3:01:15 PM & \\
\hline${ }_{4}^{\circ}$ sunflower2.dc2 & DesignCAD ... & $51424 \ldots$ & 9/9/2010 4:14:22 PM & \\
\hline$\Delta^{\circ}$ text1.dc2 & DesignCAD ... & 7638 & $10 / 4 / 201012 ; 30: 42 \ldots$ & \\
\hline D. training dc2 & DesignCAD ... & 17659 & 6/9/2010 1:52:30 PM & 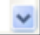 \\
\hline File Name: & & S Save & Browse & \\
\hline
\end{tabular}

Figure A-15. CAD file saving procedure and dialog box

20. Center the pattern to the origin and measure the maximum magnification to write the pattern by clicking on NPGS $\rightarrow$ MaxMag and hit the letter "o" on the keyboard to accept the centering. 

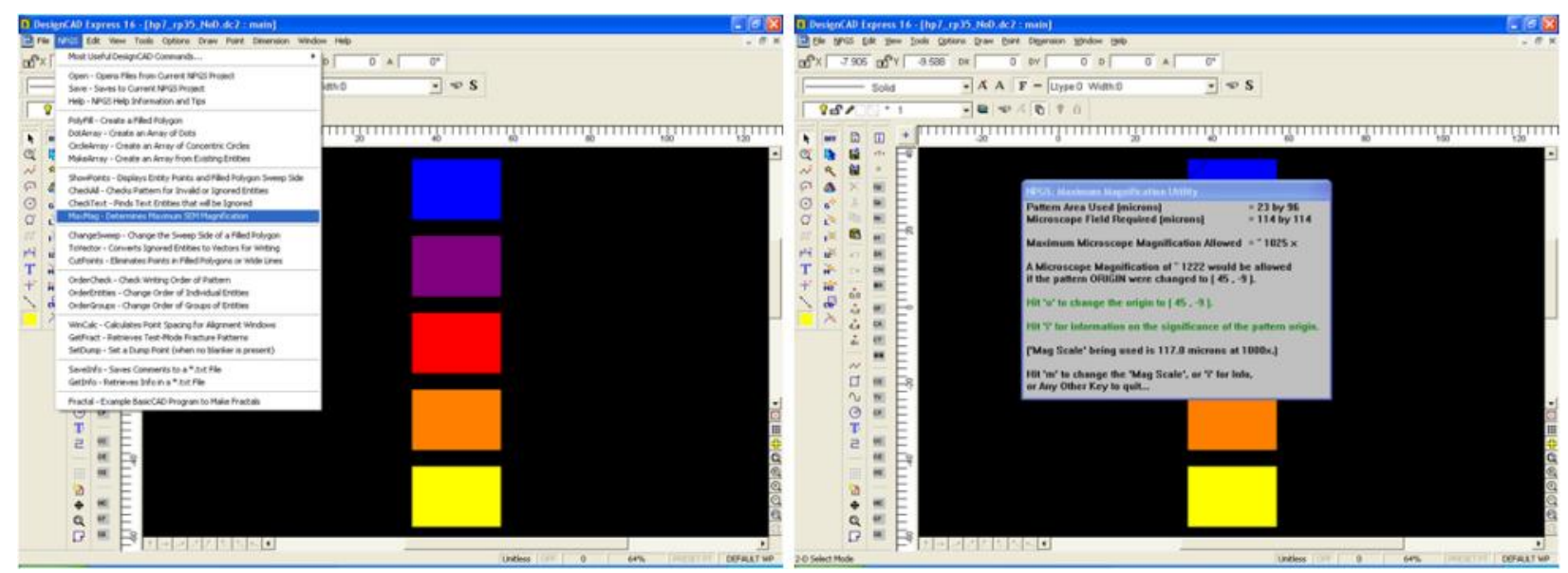

Figure A-16. MaxMag procedure of step 20. Left image indicates the location of the MaxMag command in the NPGS drop down menu. The right image indicates the dialog box that appears when the NPGS software finishes the MaxMag calculation of the designed pattern

\section{Save the changes by clicking on NPGS $\rightarrow$ Save}

22. To create a Run File, right click on the CAD file that you just created in the CAD file list of your project and the select Run File Editor.

23. Once the file completes loading, in the top right corner, select Yes to allow the pattern to be continuously writes especially when an array is select in the next step.

24. Click in the bottom left corner of the Run File Editor window and start modifying the parameters. If an array is desired, select the number of columns and rows for the pattern. Insert an initial XY movement to center the beam at a point away from the focusing spot and therefore introduce the pattern write in an unexposed region. If several $\mathrm{PhC}$ patterns were created in the DesignCAD file of different colors, each color can be assigned a different dose by changing the dose to an area dose and a number indicating the dose in units of $\mu \mathrm{C} / \mathrm{cm}^{2}$ value can be entered.

25. After all the parameters are entered for the run file, save the file and close the dialog box. The desired pattern is now ready to be written by simply clicking on Process Run File once the NPGS software is in control of the E-beam Lithography tool. 


\section{APPENDIX B: E-BEAM LITHOGRAPHY PROCESS}

Once a DesignCAD file is created, the E-beam Lithography process can be done to write the pattern. The following procedure explains this process:

1. Place the NPGS specimen holder on the SEM base.
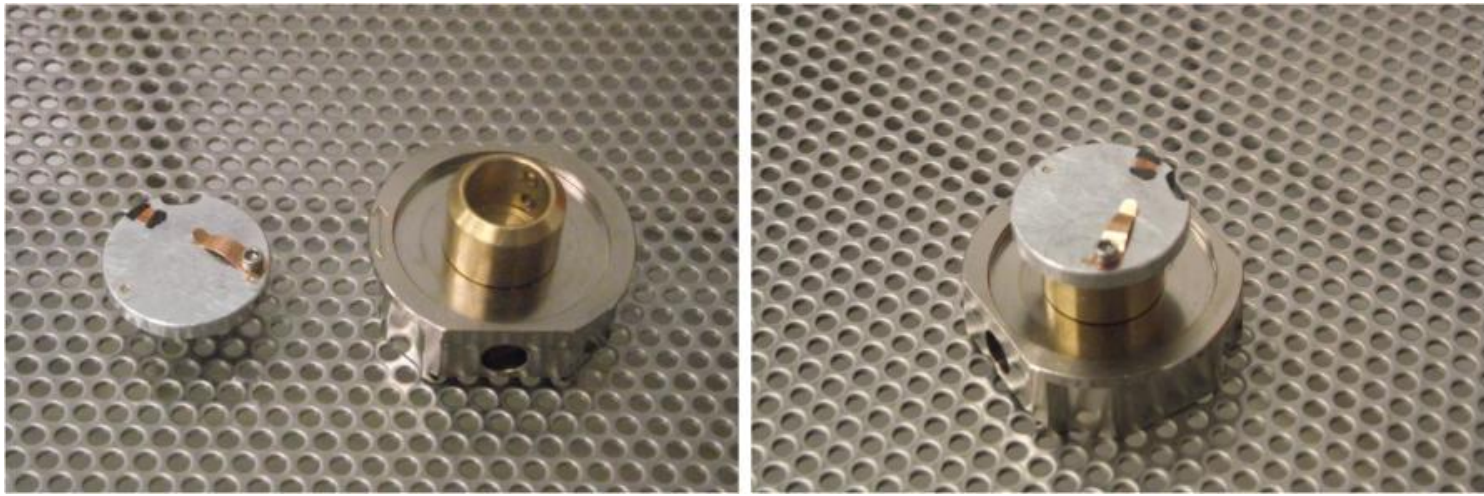

Figure B-1. The NPGS holder and its base

2. Once the desired resist is spin-coated and baked on the sample, place the sample on the holder and using a diamond scribe, make a scratch near to one of the corners of the sample.

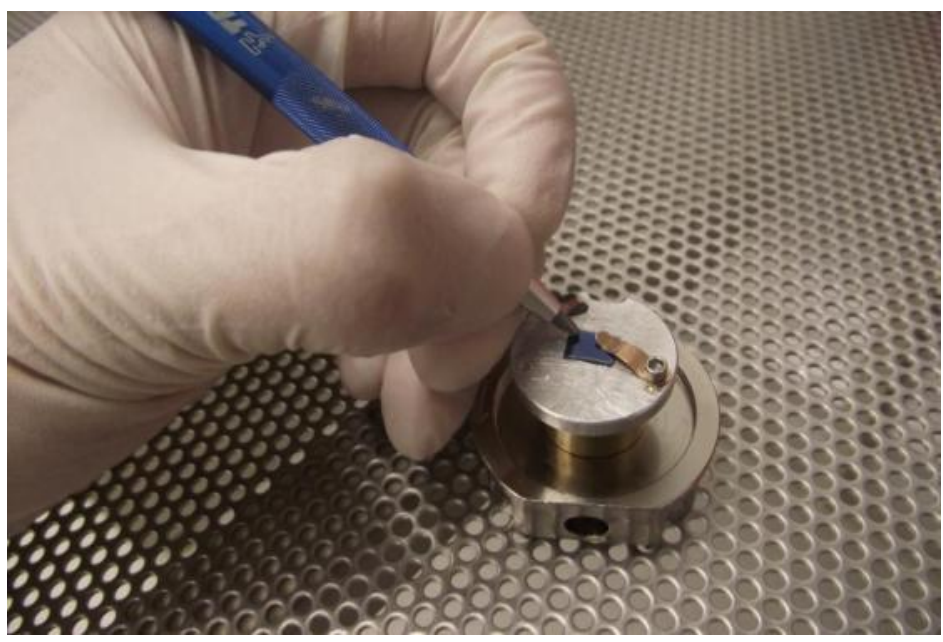

Figure B-2. Creating a small scratch in the edge of the sample for focusing purposes.

3. Vent the specimen chamber by clicking on Vent. Wait until the Vent icon turns to solid green. Open the main door of the cage, open the latch of the specimen chamber and load the sample by gently pushing 
it against the two guiding wheels. 4. Insure that the sample is properly placed and then close the door and place the latch on it.

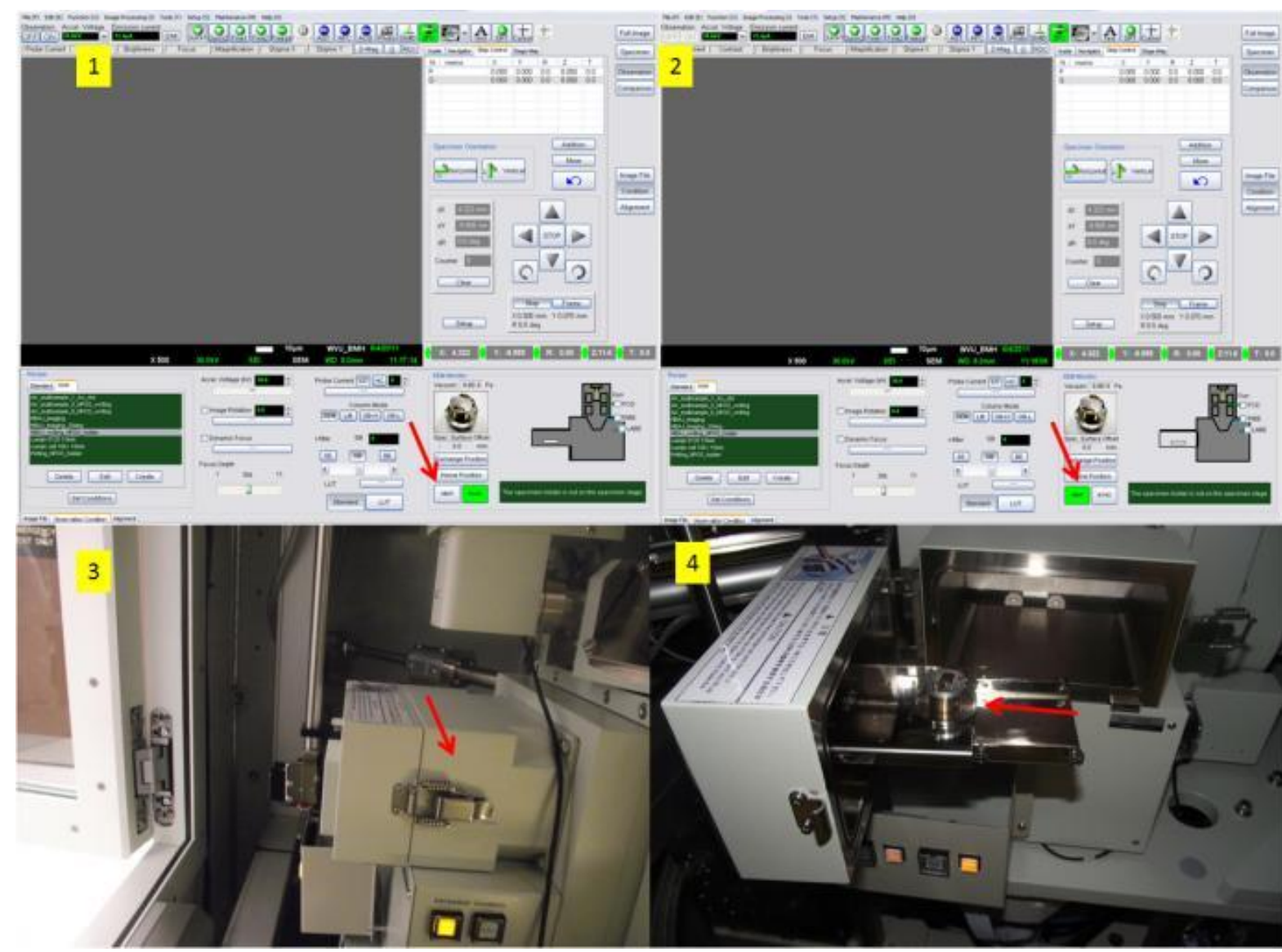

Figure B-3. Sample loading procedure. Red arrows indicate vent icon, the specimen chamber latch, and the insertion process of the sample holder.

4. Begin the pump down procedure by clicking on EVAC. Wait until the EVAC icon turns to solid green and then insert the sample by first lifting the loading rod up and then bending it to a horizontal state. Gently start pushing the rod inside the column all the way. Once loaded, the 'Select Specimen Holder" windows pops up. Select the NPGS holder and type an approximate estimate the sample thickness (usually $\sim 0.3 \mathrm{~mm}$ ). 


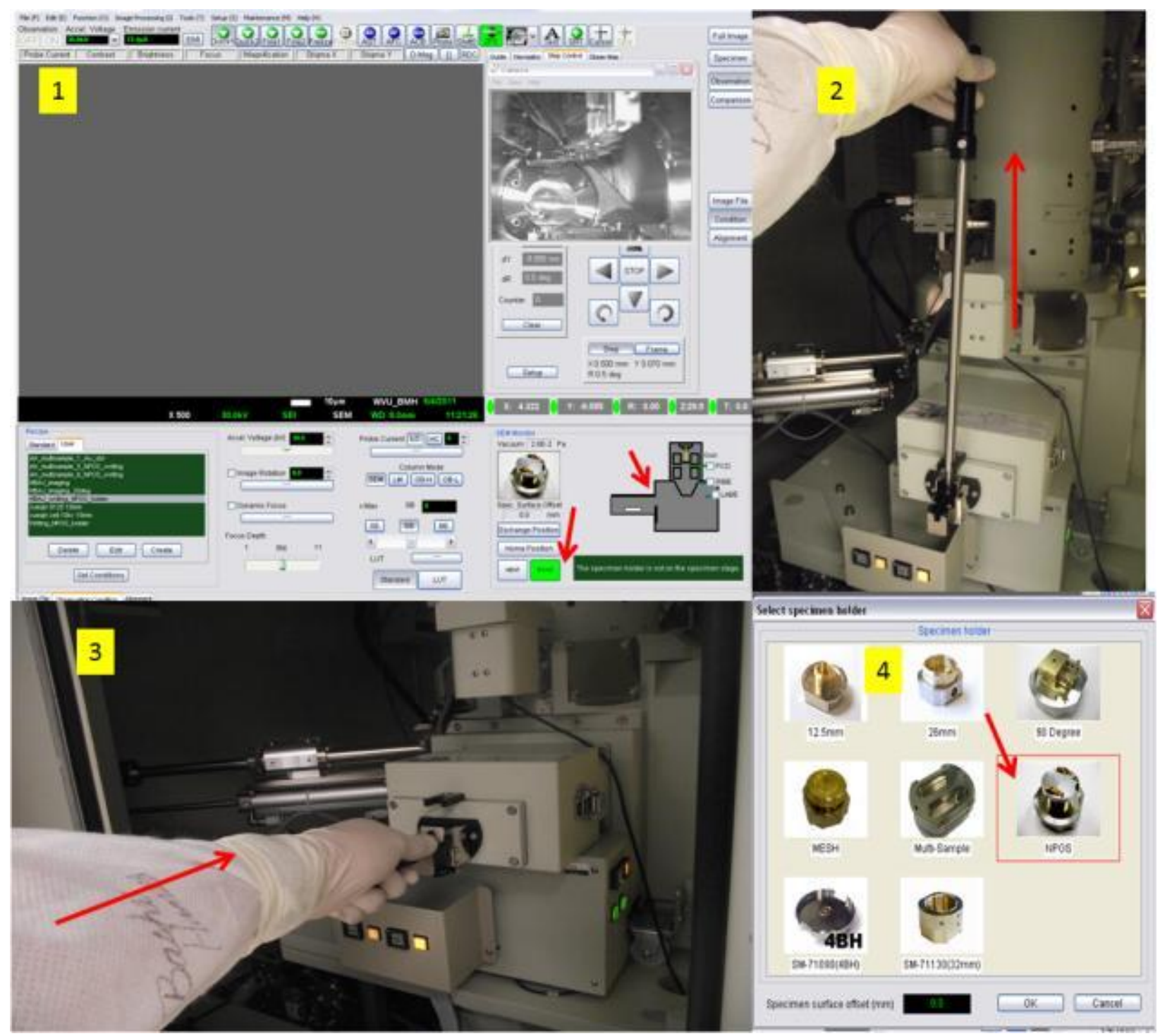

Figure B-4. Procedure to insert the sample holder into the main column.

5. Once the sample is loaded, click on the "HBAJ_Writing_NPGS_Holder" to set the X, Y, Z, Tilt, Rotation, Beam Energy, and Probe Current to the proper writing parameters. This recipe will center the Faraday cup in the center of the field view.

6. Wait until the main chamber pressure reaches to 1.9E-04 Pa. During this time, the beam blanker can be inserted and the NPGS software can be opened from the central monitor. Once at set point, the beam can now be turned by clicking ON in the top left corner of the SEM controller software. 


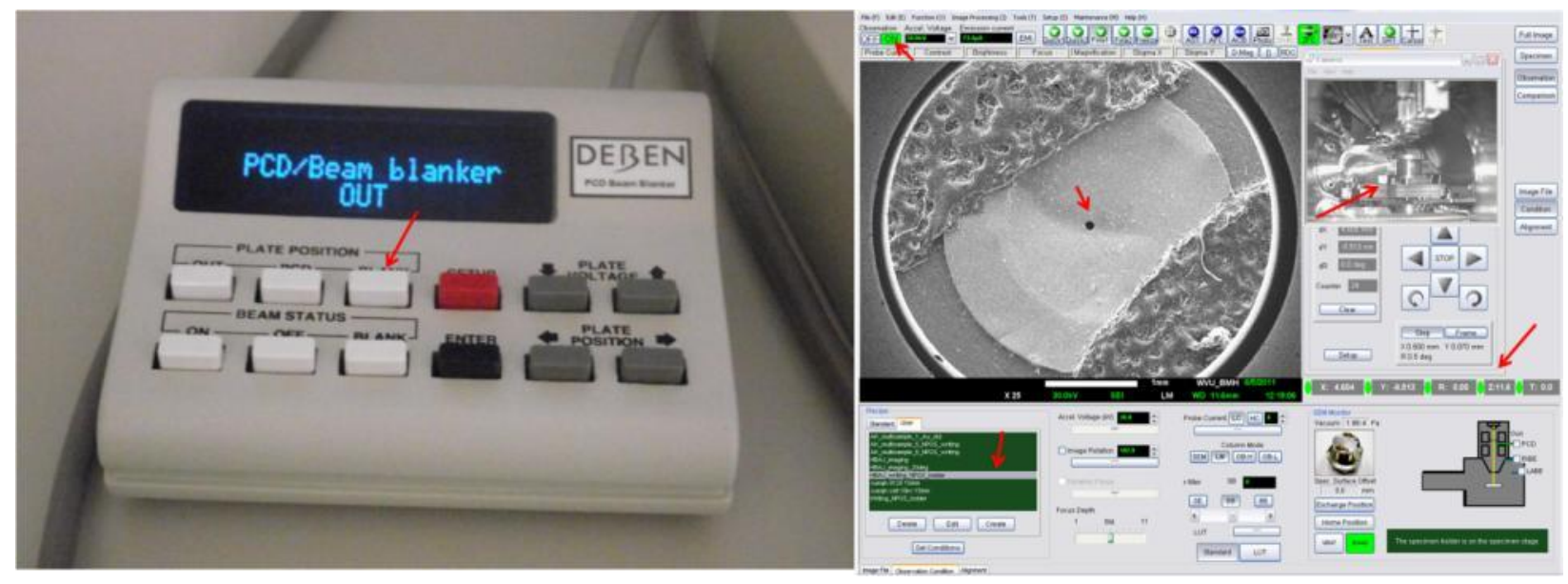

Figure B-5. Turning on the beam blanker by pushing the button indicated in the red arrow of the left image. Right image indicates the first image of the Faraday cup once the beam is turned ON in LM mode.

7. Zoom inside the Faraday Cup and turn the Cursor and the Spot (beam) to allow an accurate measure of the absorbed current value. Note this value and type it in the exposure scale box and hit enter to allow the NPGS software to automatically adjust the exposure based on the typed current value.

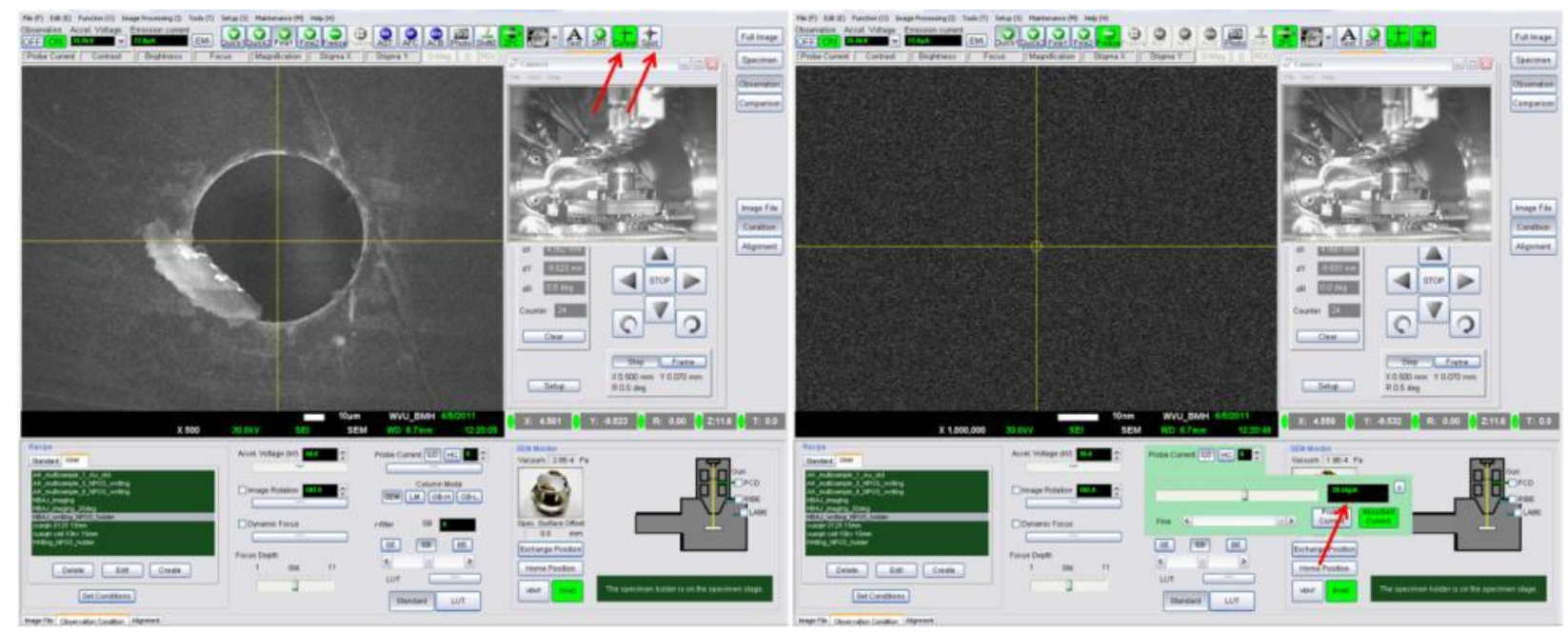

Figure B-6. Checking the probe current by collecting the beam inside the Faraday cup

8. Turn off the spot and the cursor and zoom out to the LM mode.

9. Move the stage until you find scratch and then try to locate a near-by particle. Zoom in LM until 500x and then switch to SEM mode to start focusing on the particle. 


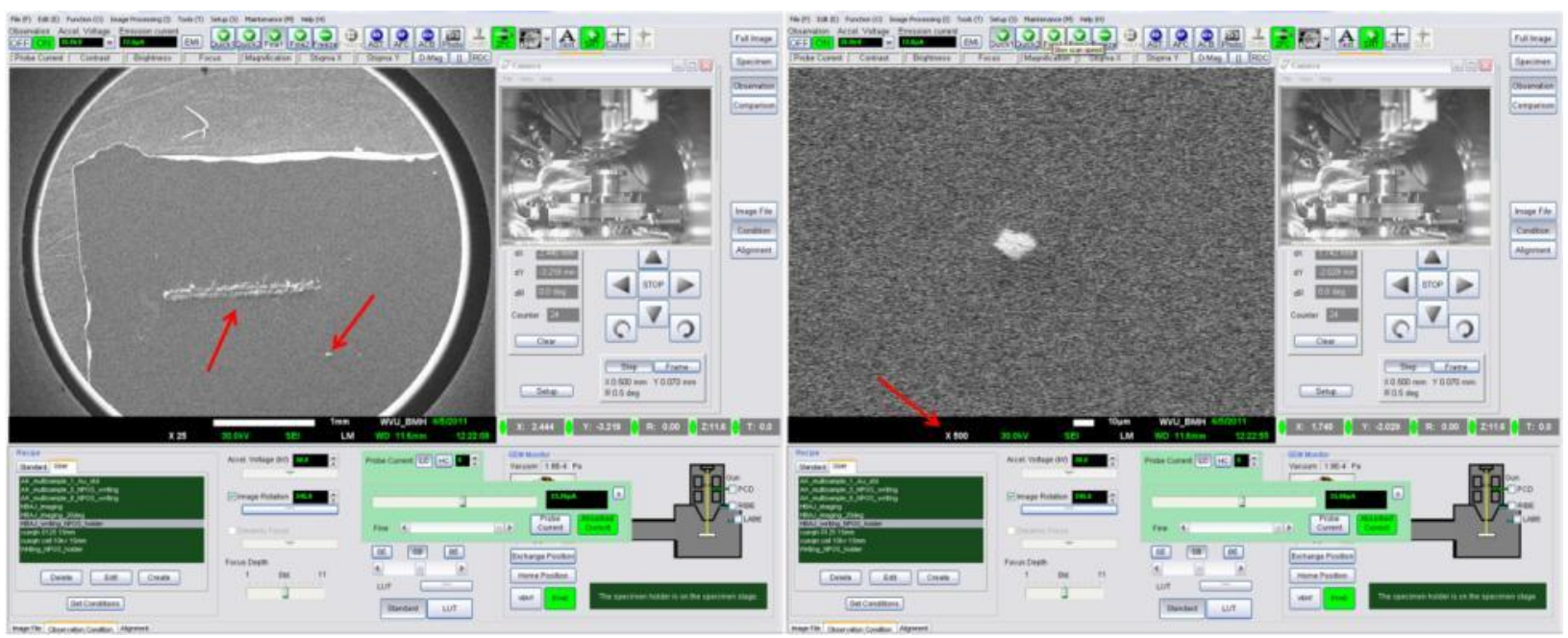

Figure B-7. The scratch area and the particle that will be used for focusing

10. Use the $\pm Z$ on the specimen stage controller to adjust the $Z$ and therefore bringing the sample within the best WD that creates the best image.

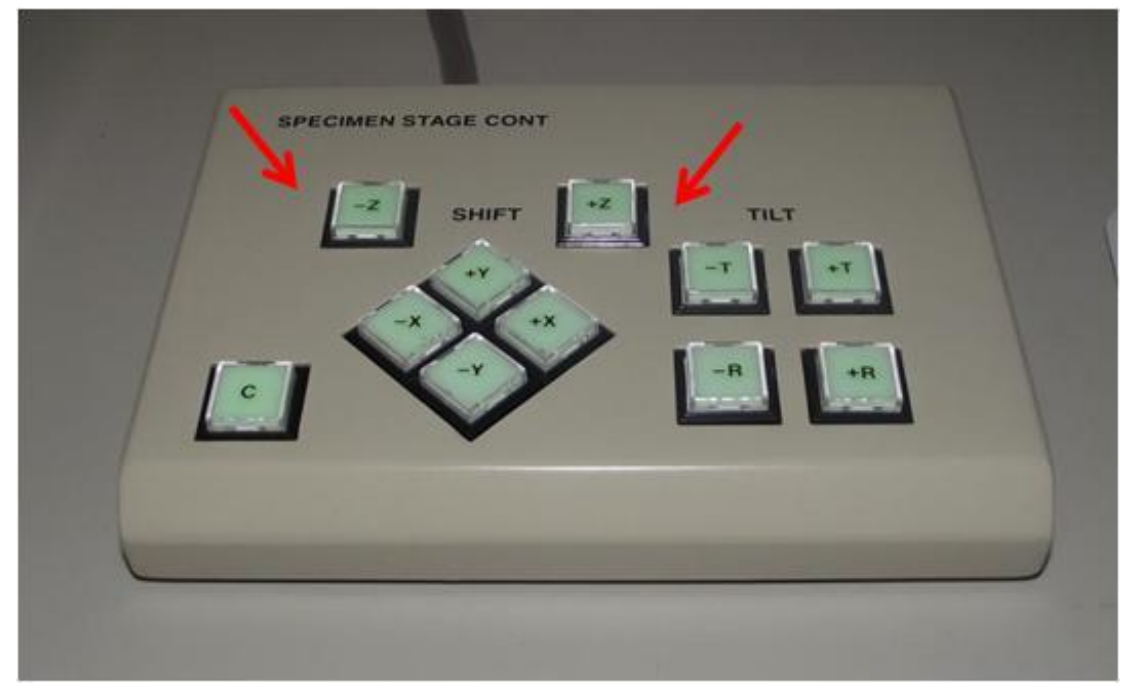

Figure B-8. Image of the Specimen Stage Controller indicating the + and $-\mathrm{Z}$ buttons

11. Keep zooming in until you reach to the $100 \mathrm{~nm}$ scale, at that scale, fine focusing is done using the focus, stigmatism $\mathrm{X}$ and $\mathrm{Y}$ knobs, and the wobbling to ensure that the beam is very well aligned. If the wobbling results a stretching in either direction, start first by adjusting the stigmatism knob in the direction of the image shift and then the orthogonal knob one at a time. Continue the focusing with increased zooming until the $10 \mathrm{~nm}$ scale. At this scale, seeing a defined edge of the particle is acceptable. 


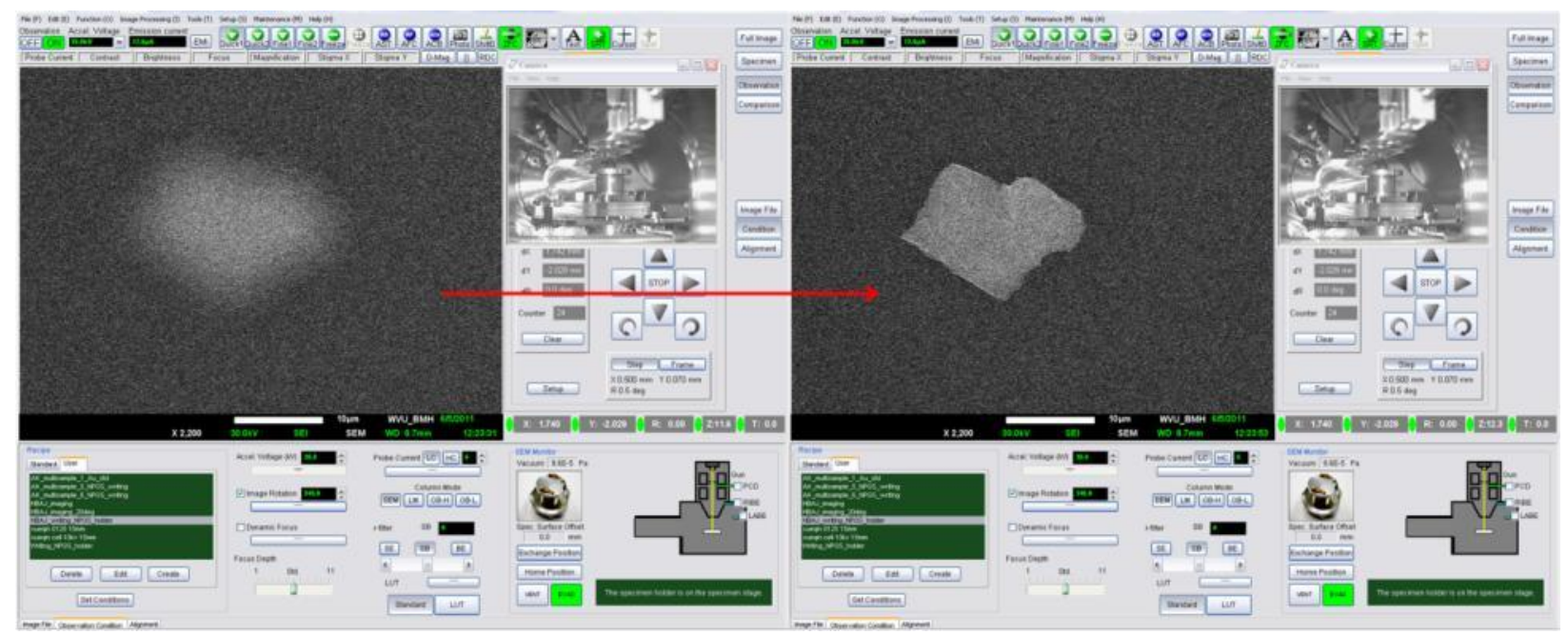

Figure B-9. The particle in SEM mode before and after the initial focusing step using the + or $-\mathrm{Z}$ buttons.

12. Move away from the particle by at least $100 \mathrm{um}$ and then zoom in to the full $100 \mathrm{~nm}$ scale bar and turn the Cursor and the Spot for at least 10 seconds to introduce a continuous exposure of the resist that will allow us to observe the beam shape.

13. Observe the shape of the exposed spot and use the focus, $\mathrm{X}$, and $\mathrm{Y}$ knobs to produce the best circular shape. You may repeat this several times until a fine well defined circle is observed with a diameter of approx. $20 \mathrm{~nm}$. For the second and third burns, reduce the exposure time to 5 and 2 seconds, respectively as the beam should be very well aligned at this stage.

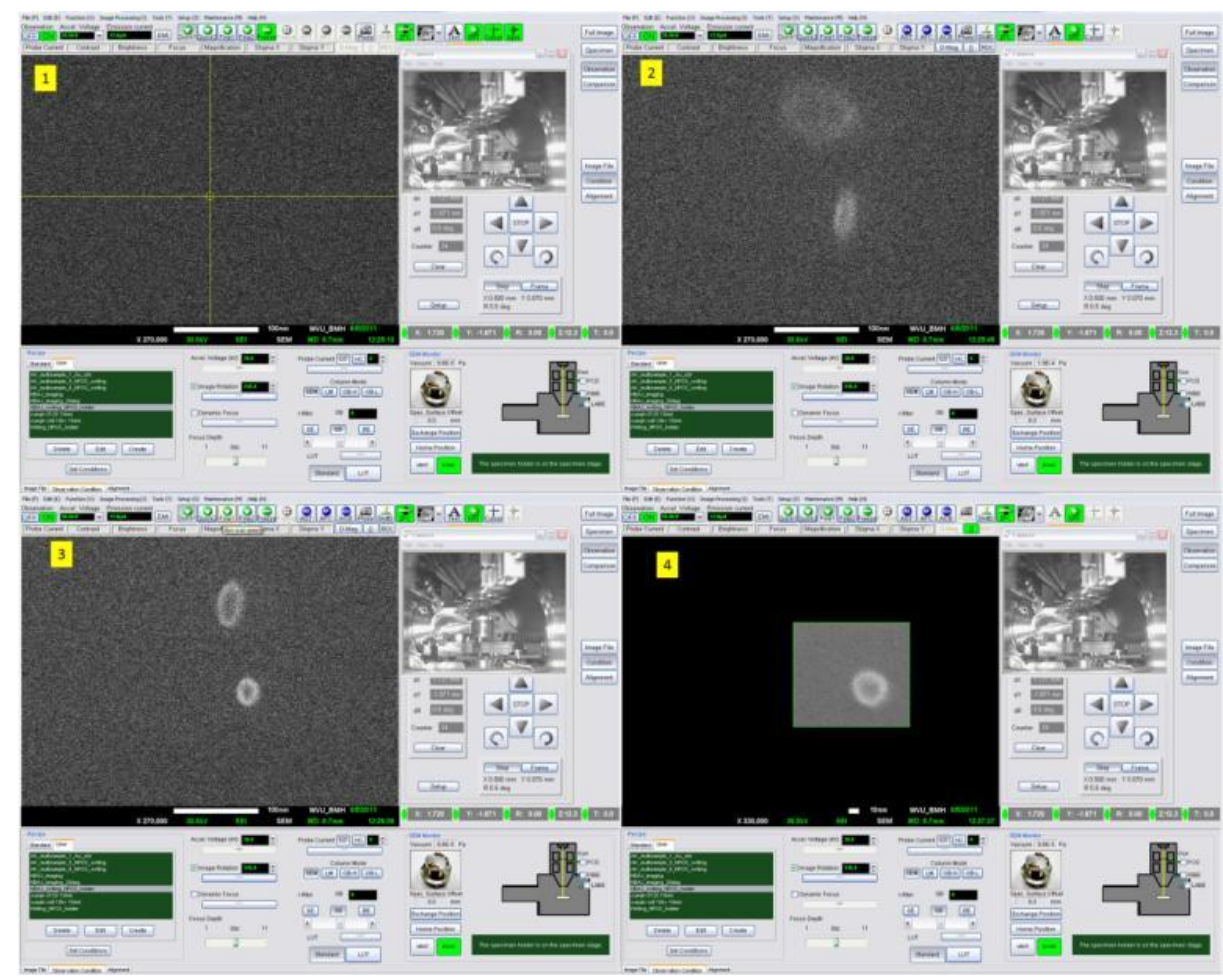

Figure B-10. Spot burning procedure to observe the beam shape. 
14. Move to the NPGS software and click on NPGS Mode to allow the NPGS to be in control of the write. Note that the Absorbed Current goes to zero if the beam blanker is inserted properly.

15. Select Process Run File to start the write.

16. Once the pattern write process is complete, turn off the beam and click on Exchange Position and switch to SEM mode in the NPGS software.

17. When the Exchange Position icon turns green, insert the rod all the way inside the chamber and pull the sample out all the way until the rod becomes outside of the guiding pipe.

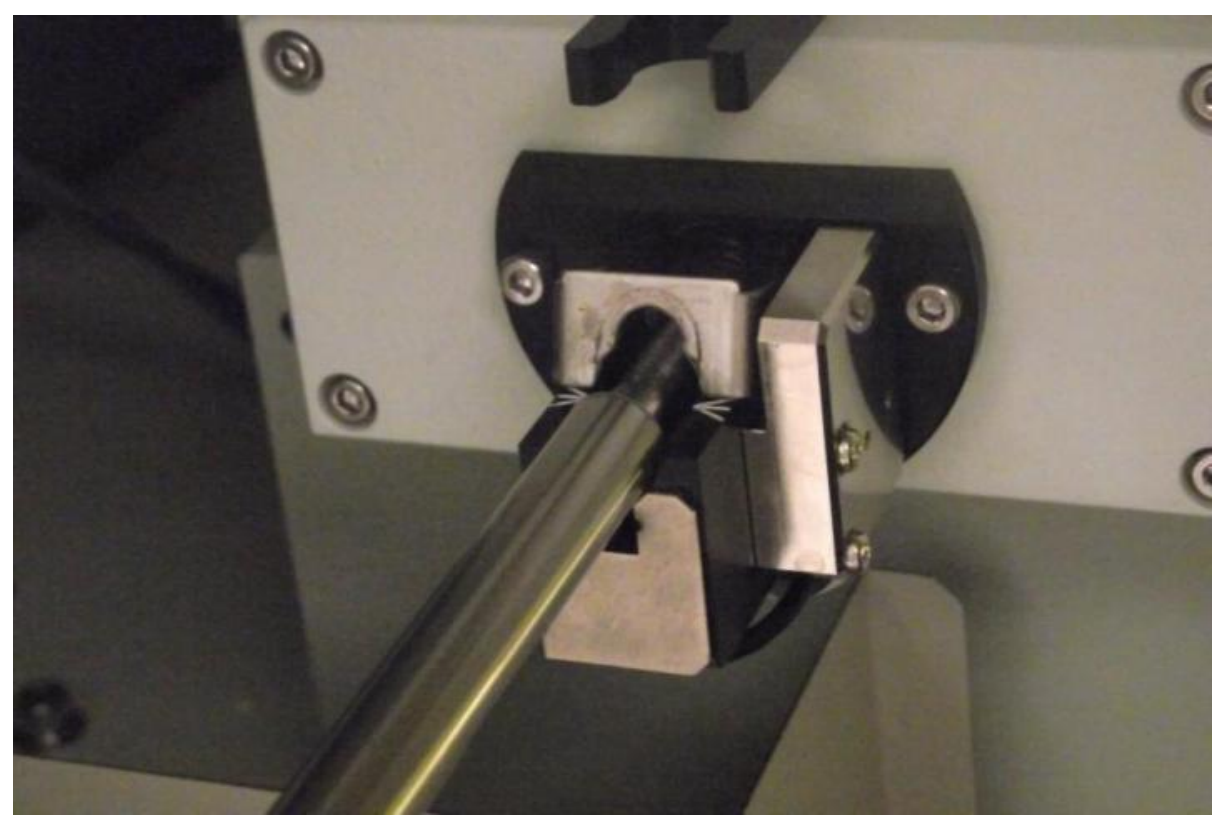

Figure B-11. A critical step of the unloading procedure in which the rod has to remain in a horizontal state when being pulled out until it passes the white arrow marks

18. Vent the specimen chamber, unload your sample, close the door, pump down by clicking on EVAC.

28. Ensure that the system is all under vacuum before leaving. 


\section{APPENDIX C: REDUCING CHARGING EFFECTS WHEN IMAGING POLYMER-BASED PHOTONIC CRYSTALS}

Imaging PDMS and Epoxy sub-micrometer features using a scanning electron microscope can be a challenging task due to the severe charge accumulation on these two bulk polymers. Regular sample grounding or sub-10-nm sputtering of a metal mask proved to be useless for this aim when characterizing these structures. Therefore, a method of metal deposition is advised.

In this method, the main purpose is to create a continuous conductive layer that extends from the sample to the holder. Here are the steps:

1. Place a carbon tape on a sputtering metal plate and place a golden cylinder sample base on top of it.

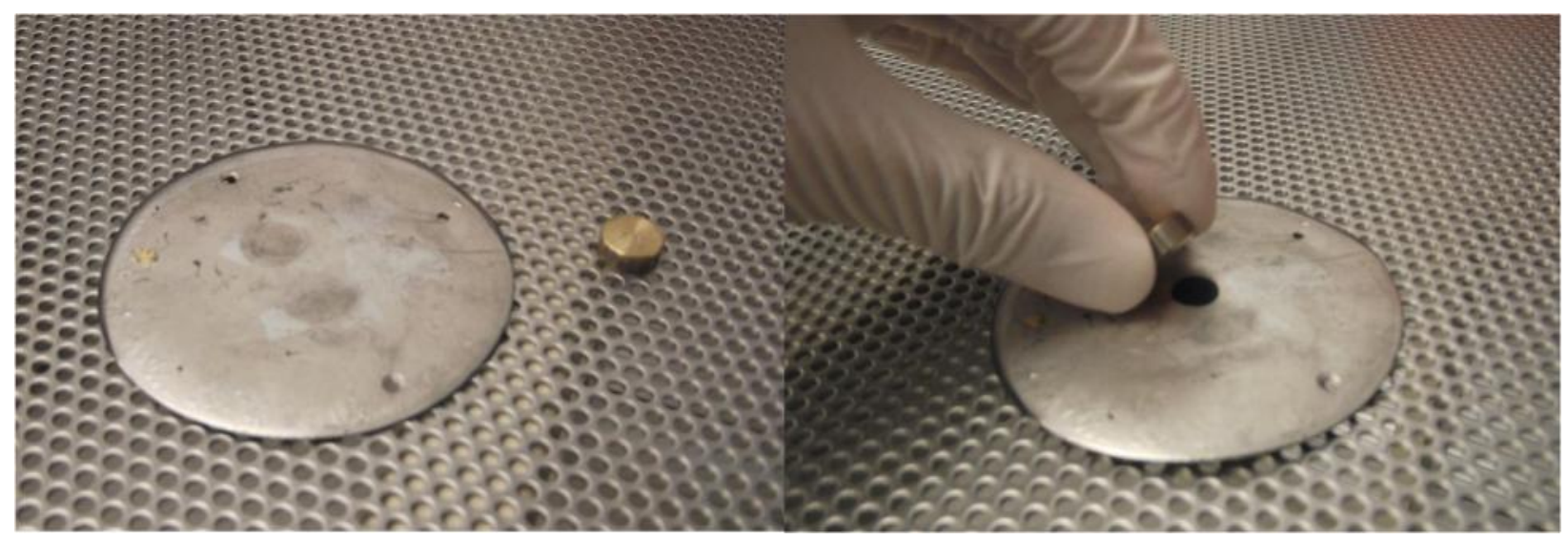

Figure C-1. Placing the SEM golden cylinder on a sputter station metal plate

2. Cut the polymer sample to be almost the same size of the golden sample bases.

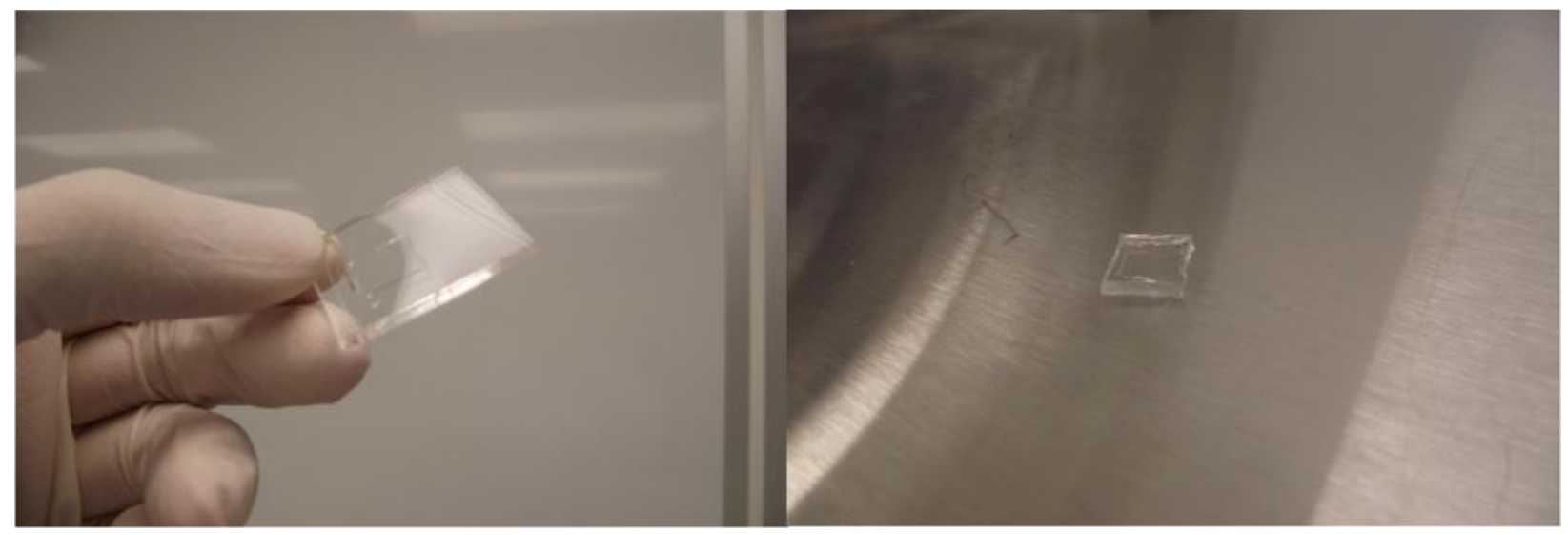

Figure C-2. Cutting the polymer sample to almost the size of the SEM golden cylinder

3. Place another tape on the golden cylinder and then gently mount the sample on the cylinder by very gently pressing on the edges surrounding the pattern area. 


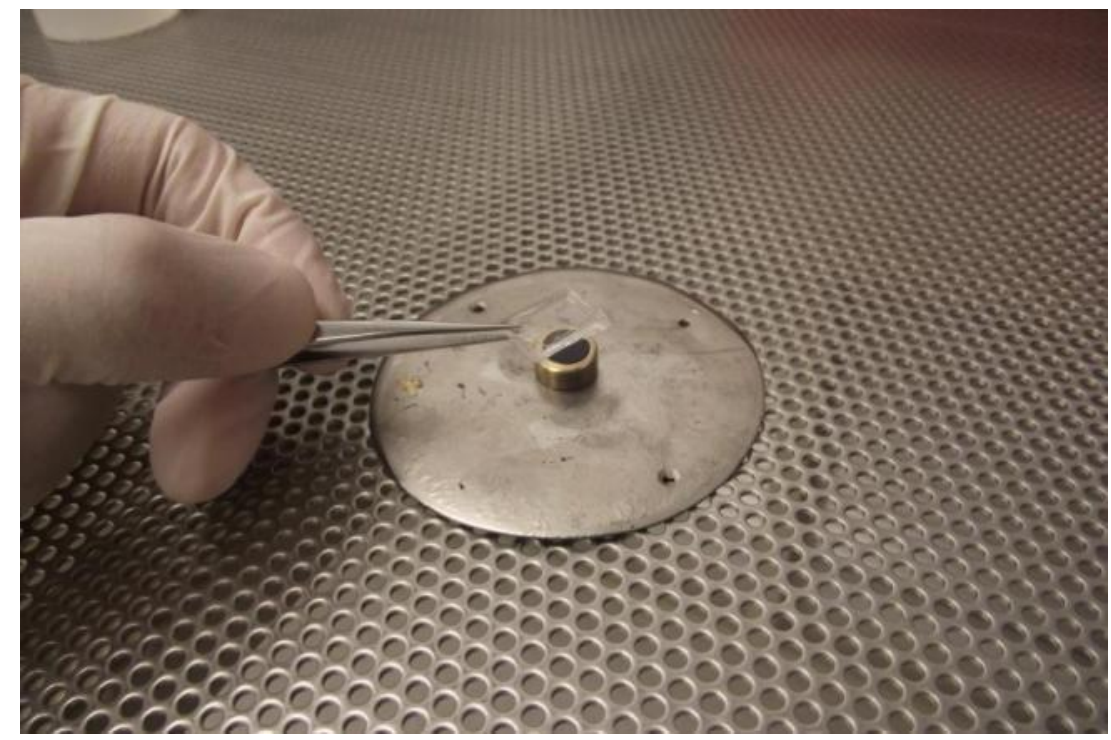

Figure C-3. Placing the sample on the golden cylinder.

4. Sputter the sample with platinum for 0.5 minute.

4. Remove the sample with its underlying golden holder from the sputtering dish slowly to insure that the sample is still in contact with the holder.

5. Place the holder with the sample inside the NPGS base and image using SEM.

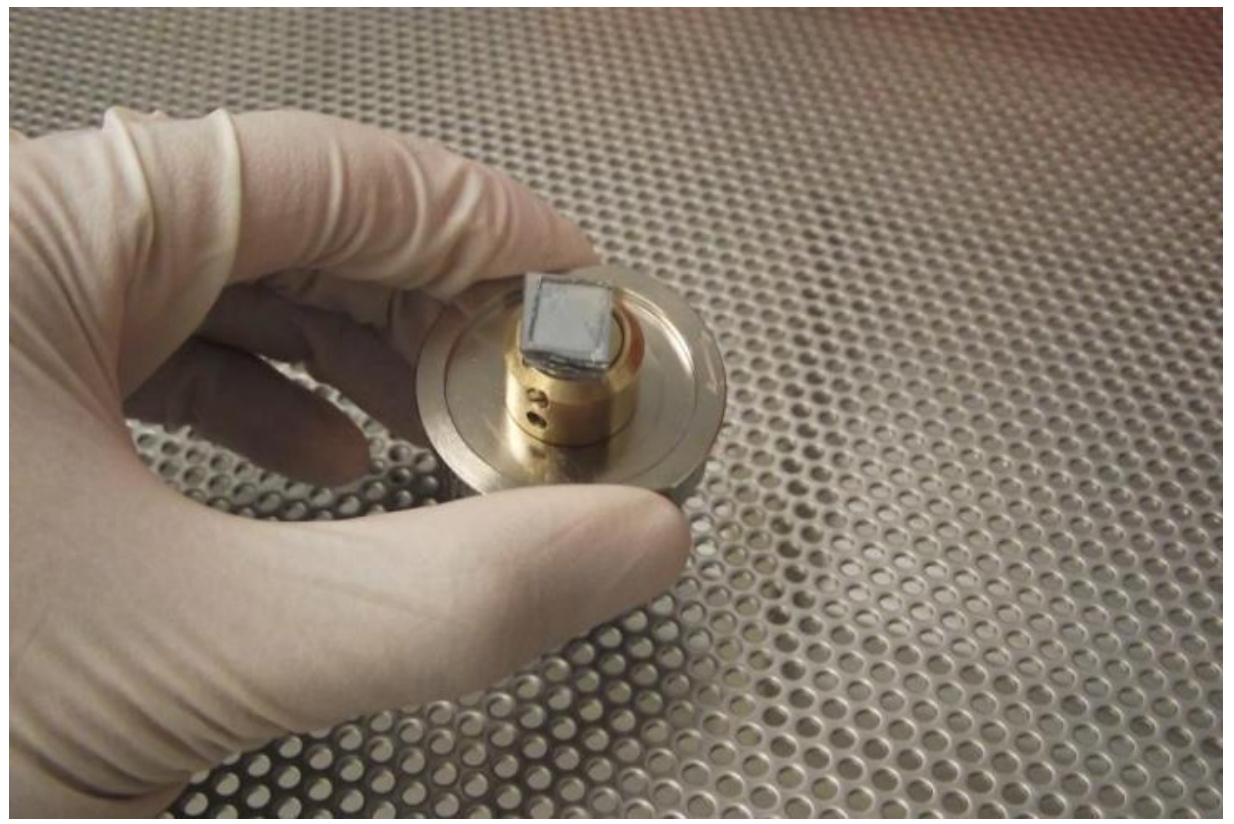

Figure C-4. The final holder after placing in it the sputtered sample with its underlying golden SEM cylinder. 


\section{APPENDIX D: MANUAL CONTROL OF THE DC BIAS}

To manually control the DC bias when etching a substrate using the Minilock-Phantom III ICP-RIE system:

1) Open the keyboard drawer cover to access the Network Tuning Controller.

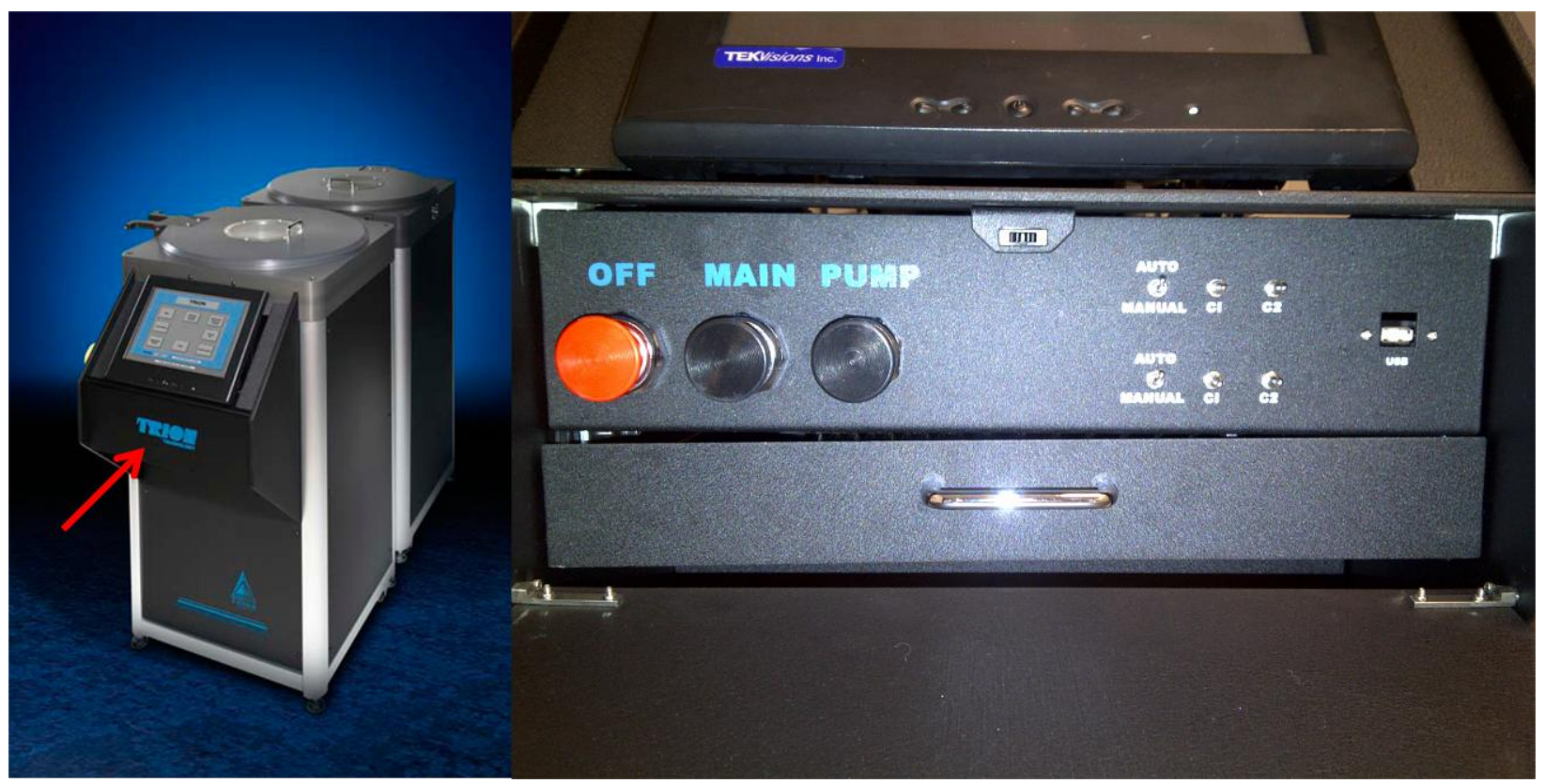

Figure D-1. The Minilock-Phantom III ICP-RIE system and the Network Tuning Controller.

2) Flip the "AUTO/MAN" switch down to the manual mode for the RIE power

3) Run the process before loading the sample and start monitoring the reflected power and the DC bias

4) Flip the bottom $\mathrm{C} 1$ and $\mathrm{C} 2$ switches up and down until the desired DC bias is observed

5) Stop the process, load your sample, and then etch using the Manual Control mode to maintain the same conditions. 